\title{
UNITARY EQUIVALENCE AND DECOMPOSITIONS OF FINITE SYSTEMS OF CLOSED DENSELY DEFINED OPERATORS IN HILBERT SPACES
}

\author{
PIOTR NIEMIEC
}

\begin{abstract}
An ideal of $N$-tuples of operators is a class invariant with respect to unitary equivalence which contains direct sums of arbitrary collections of its members as well as their (reduced) parts. New decomposition theorems (with respect to ideals) for $N$-tuples of closed densely defined linear operators acting in a common (arbitrary) Hilbert space are presented. Algebraic and order (with respect to containment) properties of the class $\mathcal{C} \mathcal{D D}_{N}$ of all unitary equivalence classes of such $N$-tuples are established and certain ideals in $\mathcal{C D} \mathcal{D}_{N}$ are distinguished. It is proved that infinite operations in $\mathcal{C D} \mathcal{D}_{N}$ may be reconstructed from the direct sum operation of a pair. Prime decomposition in $\mathcal{C D} \mathcal{D}_{N}$ is proposed and its (in a sense) uniqueness is established. The issue of classification of ideals in $\mathcal{C D} \mathcal{D}_{N}$ (up to isomorphism) is discussed. A model for $\mathcal{C D D}_{N}$ is described and its concrete realization is presented. A new partial order of $N$-tuples of operators is introduced and its fundamental properties are established. Extremal importance of unitary disjointness of $N$-tuples and the way how it 'tidies up' the structure of $\mathcal{C D} \mathcal{D}_{N}$ are emphasized.

2010 MSC: Primary 47B99; Secondary 46A10.

Key words: closed operator; densely defined operator; unitary equivalence; direct sum of operators; direct integral; decomposition of an operator; prime decomposition of an operator; finite system of operators.
\end{abstract}

\section{INTRODUCTION}

Criterions for unitary equivalence of two (bounded linear) operators (acting on Hilbert spaces) and the classification of operators up to unitary equivalence are subjects which focused an attention of many mathematicians inspired by methods and ideas proven in practice with quite well explored normal operators. The literature dealing with these and related topics is still growing up, let us mention here only a few: Brown [2] classified quasi-normal operators; Halmos and McLaughlin [17] reduced the issue of unitary equivalence of arbitrary bounded operators to partial isometries; Ernest [9], Hadwin [15, 16] and others (e.g. [21]) investigated operator-valued spectra which generalized standard (scalar) spectrum of a normal operator; Ernest [9], Brown, Fong and Hadwin [3] and Loebl [23] studied parts (that is, suboperators) of operators. It was Ernest [9] who first shown that - in a sense - the 
classification of all operators up to unitary equivalence is an essentially unattainable objective, although he gave an equivalent condition for two (totally arbitrary) bounded operators to be unitarily equivalent. It was formulated by means of certain (operator-valued) spectra of operators and multiplicity theory extended from normal to all bounded operators (roughly speaking, he adapted and generalized the classical Hahn-Hellinger theorem). The recent paper is motivated by his approach to this subject. One of aims of the paper is to finish Ernest's program of exploring the realm of unitary equivalence classes of closed densely defined operators by making no assumptions neither on the dimension of Hilbert spaces nor on boundedness of operators (this solves the problem posed by Ernest in point c of $\S 7$ of Chapter 5 of [9]). Even more, we study the class $\mathcal{C D} \mathcal{D}_{N}$ of finite systems ( $N$-tuples) of closed densely defined operators acting in (totally arbitrary) common Hilbert spaces. Surprisingly, such general considerations lead to more elegant results and reveal features which become invisible when one restricts only to separable spaces. Although $\mathcal{C D D}_{N}$ is not a set but a class, we shall show that it is 'controlled' by a single $N$-tuple (acting in a nonseparable space; cf. Proposition 9.8, page 24) and this observation will enable us to find an (algebraic as well as order) model for $\mathcal{C D D}_{N}$ (Theorem 15.2, page 46). An elementary form of the model will enable us to establish several interesting properties of $\mathcal{C D} \mathcal{D}_{N}$ (e.g. (AO13)-(AO14), page 31). Also the central decomposition introduced by Ernest for an operator acting in a separable space may be extended to a general context and translated into a more attractive (at least for us) form of the 'prime decomposition' similar to the one for natural numbers (Theorem 22.14, page 104).

Another aspect discussed in the treatise concerns various (known) results on decompositions of operators. There is a quite large number of results stating that a certain operator may be uniquely decomposed into two (or more) parts the first of which is of special type (kind, class, etc.) and the second has no nontrivial part of this type. The latter part is often named by a phrase of the form 'completely non-sth' or 'purely sth'. Let us mention only a few such results:

(DC1) a contraction operator may be decomposed into a unitary part and a completely non-unitary one,

(DC2) a bounded operator may be decomposed into a normal part and a completely non-normal one,

(DC3) a closed densely defined operator admits a unique decomposition into a normal, a purely formally normal and a completely non-formally normal part ([33])

(other results in this fashion are e.g. [10], [32], [5]). There is a striking resemblance in the above statements. And this is not a coincidence. In this paper we put all results of this type in a one general frame. To be more precise, let us introduce the notion of an ideal. It is any 
nonempty class $\mathcal{A}$ of closed densely defined operators which satisfies the following three axioms:

- if $A$ and $B$ are unitarily equivalent, then $A \in \mathcal{A} \Longleftrightarrow B \in \mathcal{A}$,

- every part (including the trivial one acting on a zero-dimensional Hilbert space) of a member of $\mathcal{A}$ belongs to $\mathcal{A}$,

- $\bigoplus_{s \in S} A_{s} \in \mathcal{A}$ whenever $\left\{A_{s}\right\}_{s \in S} \subset \mathcal{A}$ (and $S$ is a nonempty set).

For every ideal $\mathcal{A}$ we denote by $\mathcal{A}^{\perp}$ the class of all operators $A$ whose no nontrivial part belongs to $\mathcal{A}$. In Theorem 5.2 (page 11) we show that whenever $\mathcal{A}$ and $\mathcal{B}$ are ideals, so is $\mathcal{A}^{\perp}$ and every (closed densely defined) operator $T$ acting in a (completely arbitrary) Hilbert space $\mathcal{H}$ induces a unique decomposition $\mathcal{H}=\mathcal{H}_{11} \oplus \mathcal{H}_{10} \oplus \mathcal{H}_{01} \oplus \mathcal{H}_{00}$ such that $\mathcal{H}_{j k}$ are reducing subspaces for $T$ and $\left.T\right|_{\mathcal{H}_{11}} \in \mathcal{A} \cap \mathcal{B},\left.T\right|_{\mathcal{H}_{10}} \in \mathcal{A} \cap \mathcal{B}^{\perp}$, $\left.T\right|_{\mathcal{H}_{01}} \in \mathcal{A}^{\perp} \cap \mathcal{B}$ and $\left.T\right|_{\mathcal{H}_{00}} \in \mathcal{A}^{\perp} \cap \mathcal{B}^{\perp}$. This result covers (DC1)-(DC3) and all above mentioned theorems on decompositions.

Ernest [9, Definition 1.7] has introduced the notion of disjoint operators, say $A$ and $B$. In this paper we express this by writing ' $A \perp_{u} B$ ' and call $A$ and $B$ unitarily disjoint. (Unitary disjointness, as a relation, behaves as singularity of measures or orthogonality in Hilbert spaces. Beside this, unitary disjointness is formulated in order-theoretic terms in the same way as disjointness in Banach lattices, where the disjointness of two vectors $x$ and $y$ is expressed by writing $x \perp y$. This is why we prefer symbol ' $\perp_{u}$ ' for disjointness of operators than Ernest's original notation.) For Ernest the disjointness was only one of possible relations between operators. His Lebesgue decomposition of a one operator with respect to the other (Proposition 2.12 and Definition 2.13 in [9]) seems to be a secondary result rather than a 'serious' theorem. Another aim of our treatise is to underline the great importance of (unitary) disjointness (for example, we demonstrate how Ernest's central decomposition, or our prime one, may be translated into the 'intrinsic' language of operators, with use of unitary disjointness; also the proof of above mentioned Theorem 5.2 depends on properties of unitary disjointness). Roughly speaking, composing direct sums from arbitrary collections of operators is very chaotic, while the direct sum of a family of mutually unitarily disjoint operators is well 'arranged'. We may compare this with representing a simple Borel function (i.e. whose range is finite) as a linear combination of the characteristic functions of Borel sets - this may be done in infinitely many ways; there is however only one such a representation in which all appearing sets form a partition of the domain of the function. The latter form of a simple function tells us everything about the function. The same occurs in the class $\mathcal{C D} \mathcal{D}_{N}$ (see e.g. Theorem 11.1, page 26) when an $N$-tuple is written as the direct sum of a collection of mutually unitarily disjoint $N$-tuples. To distinguish between these specific decompositions and 'chaotic' ones, 
we call every direct sum (as well as any collection) of pairwise unitarily disjoint $N$-tuples regular. The notion of regularity may easily be adapt for the 'continuous' versions of direct sums (defined in Section 21 by means of direct integrals). This generalization turns out to be crucial for formulating our Prime Decomposition Theorem (Theorem 22.14, page 104).

The main tools of the treatise are, as in Ernest's work [9], techniques of von Neumann algebras. In Sections 1-17 and 23 we involve dimension theory of $\mathcal{W}^{*}$-algebras, especially a property discovered recently by Sherman [31]. All results of these sections may be formulated and proved in the language of a 'semigroup' $\operatorname{CDDD}_{N}$ with the direct sum of a pair as the only available operation (cf. Section 13). The remainder (Sections 18-22) depends on the reduction theory due to von Neumann [25]. This deals with topological and measure-theoretic aspects which are introduced in Sections 18-20. It is assumed that the reader is well oriented in basics of von Neumann algebras (it is enough to know the materials of [29], [18, 19] and [34]).

The main results of the paper are Theorems 5.2 (page 11), 11.1 (page 26), 15.2 (page 46), 22.14 (page 104) and 23.4 (page 109).

Notation and terminology. In this paper $\mathbb{R}_{+}=[0, \infty)$ and all Hilbert spaces are over the complex field. $\mathcal{H}$ and $\mathcal{K}$ denote (possibly trivial) Hilbert spaces. By an operator we mean a linear function between linear subspaces of Hilbert spaces. The Hilbert space dimension of $\mathcal{H}$ is denoted by $\operatorname{dim} \mathcal{H} . \mathcal{B}(\mathcal{H}, \mathcal{K})$ and $\mathcal{U}(\mathcal{H}, \mathcal{K})$ denote, respectively, the Banach space of all bounded operators from $\mathcal{H}$ into $\mathcal{K}$ and the set of all unitary operators of $\mathcal{H}$ onto $\mathcal{K}$, and $\mathcal{B}(\mathcal{H})=\mathcal{B}(\mathcal{H}, \mathcal{H})$ and $\mathcal{U}(\mathcal{H})=\mathcal{U}(\mathcal{H}, \mathcal{H})$. Whenever $A$ is an operator, $\mathcal{D}(A), \mathcal{R}(A), \overline{\mathcal{D}}(A)$ and $\overline{\mathcal{R}}(A)$ stand for, respectively, the domain and the range of $A$ and their closures. Additionally, $\mathcal{N}(A)$ denotes the kernel of $A$. The direct sum of a collection of Hilbert spaces $\left\{\mathcal{H}_{s}\right\}_{s \in S}$ is denoted by $\bigoplus_{s \in S} \mathcal{H}_{s}$ and $\oplus_{s} x_{s}$ is a member of $\bigoplus_{s \in S} \mathcal{H}_{s}$ corresponding to a family $\left\{x_{s}\right\}_{s \in S}$ of vectors such that $x_{s} \in \mathcal{H}_{s}$ and $\sum_{s \in S}\left\|x_{s}\right\|^{2}<\infty$. The same notation is used for direct sums of operators: if $\left\{A_{s}\right\}_{s \in S}$ is a family of operators, $A=\bigoplus_{s \in S} A_{s}$ is an operator with

$\mathcal{D}(A)=\left\{\oplus_{s} x_{s} \in \bigoplus_{s \in S} \overline{\mathcal{D}}\left(A_{s}\right): x_{s} \in \mathcal{D}\left(A_{s}\right)(s \in S), \sum_{s \in S}\left\|A_{s} x_{s}\right\|^{2}<\infty\right\}$

and for $x=\oplus_{s} x_{s} \in \mathcal{D}(A), A x=\oplus_{s}\left(A_{s} x_{s}\right) \in \bigoplus_{s \in S} \overline{\mathcal{R}}\left(A_{s}\right)$.

For two operators $A$ and $B$ acting in a common Hilbert space we write $A \subset B$ provided $\mathcal{D}(A) \subset \mathcal{D}(B)$ and $B x=A x$ for $x \in \mathcal{D}(A)$.

Let $A$ be a closed densely defined operator in $\mathcal{H}$. A closed linear subspace $E$ of $\mathcal{H}$ is said to be reducing for $A$ if $P_{E} A \subset A P_{E}$ where $P_{E}$ is the orthogonal projection onto $E$ and $\mathcal{D}\left(A P_{E}\right)=P_{E}^{-1}(\mathcal{D}(A))$. The reduced part of $A$ to $E$ is denoted by $\left.A\right|_{E}$ and it is the restriction of 
$A$ to $\mathcal{D}(A) \cap E$. The set of all reducing subspaces for $A$ is denoted by $\operatorname{red}(A) . \quad E \in \operatorname{red}(A)$ is centrally reducing iff $P_{E} P_{K}=P_{K} P_{E}$ for any $K \in \operatorname{red}(A)$. The collection of all centrally reducing subspaces is denoted by $\operatorname{cred}(A)$. The $*$-commutant of $A$ is the set $\mathcal{W}^{\prime}(A)$ consisting of all $T \in \mathcal{B}(\mathcal{H})$ such that $T A \subset A T$ and $T^{*} A \subset A T^{*}$; and $\mathcal{W}^{\prime \prime}(A)=$ $\left(\mathcal{W}^{\prime}(A)\right)^{\prime}$ is the $*$-bicommutant of $A$. When $A$ is bounded, we may also use $\mathcal{W}(A)$ to denote the smallest von Neumann algebra containing $A$, in that case $\mathcal{W}(A)=\mathcal{W}^{\prime \prime}(A)$ (thanks to von Neumann's bicommutant theorem). The polar decomposition of $A$ has the form $A=Q|A|$ where $|A|$ is the square root of $A^{*} A$ (obtained e.g. by the functional calculus for unbounded selfadjoint operators) and $Q$ is a partial isometry with $\mathcal{N}(Q)=\mathcal{N}(A)$. Whenever we use the notation ' $Q_{T}$ ' with $T$ being a closed densely defined operator, this denotes the partial isometry appearing in the polar decomposition of $T$.

\section{Preliminaries}

In the whole paper, $N$ is a fixed positive integer corresponding to the length of tuples of operators. Whenever $\mathcal{H}$ is a Hilbert space, $\operatorname{CDD}(\mathcal{H})$ is the collection of all closed densely defined linear operators acting in $\mathcal{H}$ and $\operatorname{CDD}_{N}(\mathcal{H})=[\mathrm{CDD}(\mathcal{H})]^{N}$. That is, $\operatorname{CDD}_{N}(\mathcal{H})$ consists of all $N$-tuples of members of $\operatorname{CDD}(\mathcal{H})$. Further, we put

$$
\mathrm{CDD}_{N}=\bigcup_{\mathcal{H}} \operatorname{CDD}_{N}(\mathcal{H})
$$

where $\mathcal{H}$ runs over all Hilbert spaces (including zero-dimensional). For simplicity, we shall write CDD in place of $\mathrm{CDD}_{1}$. For every $\boldsymbol{A}=$ $\left(A_{1}, \ldots, A_{N}\right) \in \mathrm{CDD}_{N}$ there is a unique Hilbert space, denoted by $\overline{\mathcal{D}}(\boldsymbol{A})$, such that $\boldsymbol{A} \in \mathrm{CDD}_{N}(\overline{\mathcal{D}}(\boldsymbol{A}))$. In particular, $\overline{\mathcal{D}}(\boldsymbol{A})=\overline{\mathcal{D}}\left(A_{j}\right)$ for $j=1, \ldots, N$.

Suppose $\boldsymbol{A}=\left(A_{1}, \ldots, A_{N}\right) \in \mathrm{CDD}_{N}$. We define $\boldsymbol{A}^{*},|\boldsymbol{A}|$ and $\boldsymbol{Q}_{\boldsymbol{A}}$ (as members of $\left.\mathrm{CDD}_{N}\right)$ in a coordinatewise manner: $\boldsymbol{A}^{*}=\left(A_{1}^{*}, \ldots, A_{N}^{*}\right)$, $|\boldsymbol{A}|=\left(\left|A_{1}\right|, \ldots,\left|A_{N}\right|\right)$ and $\boldsymbol{Q}_{\boldsymbol{A}}=\left(Q_{A_{1}}, \ldots, Q_{A_{N}}\right)$. In the same way we may define other operations on $N$-tuples, if only they can be made on each of their entries. For example, if each of $A_{j}$ 's is one-to-one and has dense image, we may define $\boldsymbol{A}^{-1}$ as $\left(A_{1}^{-1}, \ldots, A_{N}^{-1}\right)$.

Everywhere below in points (DF1)-(DF11), $\boldsymbol{A}=\left(A_{1}, \ldots, A_{N}\right), \boldsymbol{B}=$ $\left(B_{1}, \ldots, B_{N}\right)$ and $\boldsymbol{A}^{(s)}=\left(A_{1}^{(s)}, \ldots, A_{N}^{(s)}\right)$ represent arbitrary members of $\mathrm{CDD}_{N}$. For a single operator, some of notions stated below are well-known and some of them were introduced in [9] (with different notation). Probably the only new notion is the strong order ' $\leqslant$ ' defined in (DF8) below.

(DF1) Let $\bigoplus_{s \in S} A^{(s)}=\left(\bigoplus_{s \in S} A_{1}^{(s)}, \ldots, \bigoplus_{s \in S} A_{N}^{(s)}\right)$. For a positive cardinal $\alpha$ let $\alpha \odot A=\bigoplus_{\xi<\xi_{\alpha}} A^{(\xi)}$ where $\xi_{\alpha}$ is the first ordinal of cardinality $\alpha$ and $\boldsymbol{A}^{(\xi)}=\boldsymbol{A}$ for any $\xi<\xi_{\alpha}$. 
(DF2) $\boldsymbol{A}$ is trivial provided $\overline{\mathcal{D}}(\boldsymbol{A})$ is zero-dimensional; otherwise $\boldsymbol{A}$ is nontrivial.

(DF3) $A$ is bounded iff each of $A_{1}, \ldots, A_{N}$ is a bounded operator; $\|\boldsymbol{A}\|:=\max \left(\left\|A_{1}\right\|, \ldots,\left\|A_{N}\right\|\right)$ provided $\boldsymbol{A}$ is bounded, otherwise $\|\boldsymbol{A}\|:=\infty$. We say a bounded $\boldsymbol{A}$ assumes its norm provided there is $x \in \overline{\mathcal{D}}(\boldsymbol{A})$ of norm 1 with $\max \left(\left\|A_{1} x\right\|, \ldots,\left\|A_{N} x\right\|\right)=$ $\|A\|$.

(DF4) Let $\operatorname{red}(\boldsymbol{A})=\bigcap_{j=1}^{N} \operatorname{red}\left(A_{j}\right)$ and for $E \in \operatorname{red}(\boldsymbol{A})$,

$$
\left.\boldsymbol{A}\right|_{E}=\left(\left.A_{1}\right|_{E}, \ldots,\left.A_{N}\right|_{E}\right)
$$

$\operatorname{cred}(\boldsymbol{A})$ consists of all $E \in \operatorname{red}(\boldsymbol{A})$ such that $P_{E} P_{K}=P_{K} P_{E}$ for every $K \in \operatorname{red}(\boldsymbol{A})$.

(DF5) The *-commutant of $A$ is the set $\mathcal{W}^{\prime}(A)=\bigcap_{j=1}^{N} \mathcal{W}^{\prime}\left(A_{j}\right) \subset$ $\mathcal{B}(\overline{\mathcal{D}}(\boldsymbol{A}))$ and $\mathcal{W}^{\prime \prime}(\boldsymbol{A})=\left(\mathcal{W}^{\prime}(\boldsymbol{A})\right)^{\prime}$ is the $*$-bicommutant of $\boldsymbol{A}$. When $\boldsymbol{A}$ is bounded, we may also use $\mathcal{W}(A)$ to denote the smallest von Neumann algebra including $\left\{A_{1}, \ldots, A_{N}\right\}$, in that case $\mathcal{W}(A)=\mathcal{W}^{\prime \prime}(A)$

(DF6) $\boldsymbol{A} \equiv \boldsymbol{B}$ (or, $\boldsymbol{A}$ and $\boldsymbol{B}$ are unitarily equivalent) iff there is $U \in$ $\mathcal{U}(\overline{\mathcal{D}}(\boldsymbol{A}), \overline{\mathcal{D}}(\boldsymbol{B}))$ such that $A_{j}=U^{-1} B_{j} U$ for $j=1, \ldots, N$.

(DF7) $\boldsymbol{A} \leqslant \boldsymbol{B}$ iff $\left.\boldsymbol{A} \equiv \boldsymbol{B}\right|_{E}$ for some $E \in \operatorname{red}(\boldsymbol{B})$.

(DF8) $\boldsymbol{A} \leqslant \leqslant^{s} \boldsymbol{B}$ iff $\left.\boldsymbol{A} \equiv \boldsymbol{B}\right|_{E}$ for some $E \in \operatorname{cred}(\boldsymbol{B})$.

(DF9) $\boldsymbol{A}$ and $\boldsymbol{B}$ are unitarily disjoint, in symbol $\boldsymbol{A} \perp_{u} \boldsymbol{B}$, if there is no nontrivial $N$-tuple $\boldsymbol{X} \in \mathrm{CDD}_{N}$ with $\boldsymbol{X} \leqslant \boldsymbol{A}$ and $\boldsymbol{X} \leqslant \boldsymbol{B}$.

(DF10) $\boldsymbol{A}$ is covered by $\boldsymbol{B}$, in symbol $\boldsymbol{A} \ll \boldsymbol{B}$, if $\boldsymbol{A} \leqslant \alpha \odot \boldsymbol{B}$ for some cardinal $\alpha$.

(DF11) The symbols ' $\boxplus$ ' and ' $\boxplus$ ' shall often be used instead of ' $\oplus$ ' and ' $\bigoplus$ ' in situations when all summands are mutually unitarily disjoint. So, whenever in the sequel notation $\boldsymbol{A} \boxplus \boldsymbol{B}$ or $\boxplus_{s \in S} \boldsymbol{A}^{(s)}$ appears, this will always imply that $A \perp_{u} B$ or, respectively, $\boldsymbol{A}^{\left(s^{\prime}\right)} \perp_{u} \boldsymbol{A}^{\left(s^{\prime \prime}\right)}$ for any distinct indices $s^{\prime}, s^{\prime \prime} \in S$. The direct sum (a collection) is called regular provided all its summands (elements) are mutually unitarily disjoint.

The reader should notice that a function $\operatorname{red}(A) \ni E \mapsto P_{E} \in \mathcal{W}^{\prime}(A)$ establishes a one-to-one correspondence between $\operatorname{red}(\boldsymbol{A})$ and the set $E\left(\mathcal{W}^{\prime}(A)\right)$ of all orthogonal projections belonging to $\mathcal{W}^{\prime}(A)$. What is more, this map sends $\operatorname{cred}(A)$ onto $E\left(\mathcal{W}^{\prime}(A)\right) \cap \mathcal{Z}\left(\mathcal{W}^{\prime}(A)\right)$ where $\mathcal{Z}\left(\mathcal{W}^{\prime}(\boldsymbol{A})\right)$ is the center of $\mathcal{W}^{\prime}(\boldsymbol{A})$.

It is quite easy to check that ' $\equiv$ ' is an equivalence on $\mathrm{CDD}_{N}$ and thus for each $A \in \mathrm{CDD}_{N}$ we may consider the equivalence class of $\boldsymbol{A}$ with respect to ' $\equiv$ ', which we shall denote by $\mathbb{A}$. Let $\mathcal{C D} \mathcal{D}_{N}$ be the class of (all) equivalence classes of all members of $\mathrm{CDD}_{N}$ and let $\mathcal{C D D}=\mathcal{C D D}_{1}$. Elements of $\mathcal{C D D}_{N}$ will be denoted by $\mathbb{A}, \mathbb{B}, \mathbb{X}, \mathbb{Y}$ and so on and their corresponding representatives by $A, B, X, Y$. The symbol $\mathbb{O}$ is reserved to denote the equivalence class of a trivial element 
of $\mathrm{CDD}_{N}$. $\mathbb{O}$ is the unique member of $\mathcal{C D D}_{N}$ whose representatives act on zero-dimensional Hilbert spaces. (It is also the neutral element for the action ' $\oplus$ '.) For every $\mathbb{A} \in \mathcal{C D} \mathcal{D}_{N}$, the following are well defined, in an obvious manner: $\mathbb{A}^{*},|\mathbb{A}|, \mathbb{Q}_{\mathbb{A}}$ (the latter corresponds to $\boldsymbol{Q}_{\boldsymbol{A}}$ ) and $\operatorname{dim}(\mathbb{A})=\operatorname{dim} \overline{\mathcal{D}}(\boldsymbol{A})$. For simplicity, we shall use the term ' $N$-tuple' for members of $\mathrm{CDD}_{N}$ as well as for members of $\mathrm{CDD}_{N}$.

Some of notions in (DF1)-(DF11) may be adapted for members of $\operatorname{eDD}_{N}$ as follows.

(UE1) Let $\bigoplus_{s \in S} \mathbb{A}^{(s)}=\mathbb{X}$ where $\boldsymbol{X}=\bigoplus_{s \in S} \boldsymbol{A}^{(s)}$. For any cardinal $\mathfrak{m}>0$, put $\mathfrak{m} \odot \mathbb{A}=\mathbb{Y}$ where $\boldsymbol{Y}=\mathfrak{m} \odot \boldsymbol{A}$. Additionally, let $0 \odot \mathbb{A}=\mathbb{O}$.

(UE2) $\mathbb{A}$ is bounded, nontrivial, trivial iff so is $A$. $\|\mathbb{A}\|=\|A\| ; \mathbb{A}$ assumes its norm iff so does $A$.

(UE3) $\mathbb{A} \leqslant \mathbb{B}, \mathbb{A} \leqslant^{s} \mathbb{B}, \mathbb{A} \perp_{u} \mathbb{B}, \mathbb{A} \ll \mathbb{B}$ iff corresponding relation holds true for $A$ and $B$. Note that $\mathbb{A} \leqslant^{s} \mathbb{B} \Longrightarrow \mathbb{A} \leqslant \mathbb{B} \Longrightarrow \mathbb{A} \ll \mathbb{B}$.

(UE4) Notation $\mathbb{A} \boxplus \mathbb{B}$ or $\boxplus_{s \in S} \mathbb{A}^{(s)}$ includes information that $\mathbb{A} \perp_{u} \mathbb{B}$ or, respectively, $\mathbb{A}^{\left(s^{\prime}\right)} \perp_{u} \mathbb{A}^{\left(s^{\prime \prime}\right)}$ for any distinct indices $s^{\prime}, s^{\prime \prime} \in S$. The direct sum (a family) of members of $\mathcal{C D D}_{N}$ is regular iff all its summands (elements) are pairwise unitarily disjoint.

A starting point for all of our investigations is the following classical result (see e.g. [9, Theorem 1.3]).

2.1. Proposition. ' $\leqslant$ ' and ' $\leqslant$ ' are partial orders on $\mathcal{C D} \mathcal{D}_{N}$. More precisely, if $\mathbb{A} \leqslant \mathbb{B}$ and $\mathbb{B} \leqslant \mathbb{A}$, then $\mathbb{A}=\mathbb{B}$.

\section{THE $\mathfrak{b}$-TRANSFORM}

This part is mainly devoted to single operators.

We fix a Hilbert space $\mathcal{H}$ and an operator $T \in \operatorname{CDD}(\mathcal{H})$. Let $I$ be the identity operator on $\mathcal{H}$.

3.1. Definition. The $\mathfrak{b}$-transform of $T$ is the operator

$$
\mathfrak{b}(T)=T(I+|T|)^{-1} \in \mathcal{B}(\mathcal{H}) .
$$

The reader should verify with no difficulties that

3.2. Proposition. Let $S=\mathfrak{b}(T)$.

(A) $\mathfrak{b}(|T|)=|S|=|T|(I+|T|)^{-1}$ and $Q_{T}=Q_{S}$.

(B) $\|S x\|<\|x\|$ for each $x \in \mathcal{H} \backslash\{0\}$.

(C) $T=S(I-|S|)^{-1}=: \mathfrak{u} \mathfrak{b}(S)$.

(D) $\mathcal{W}^{\prime}(T)=\mathcal{W}^{\prime}(S)$. Consequently, $\operatorname{red}(T)=\operatorname{red}(S)$ and $\operatorname{cred}(T)=$ $\operatorname{cred}(S)$. For every $E \in \operatorname{red}(T), \mathfrak{b}\left(\left.T\right|_{E}\right)=\left.S\right|_{E}$.

(E) The $\mathfrak{b}$-transform establishes a one-to-one correspondence between members of $\operatorname{CDD}(\mathcal{H})$ and operators $S \in \mathcal{B}(\mathcal{H})$ satisfying (B).

(F) $\mathfrak{b}\left(\bigoplus_{s \in S} T_{s}\right)=\bigoplus_{s \in S} \mathfrak{b}\left(T_{s}\right)$ for arbitrary family $\left\{T_{s}\right\}_{s \in S} \subset \mathrm{CDD}$.

The following result is a little bit surprising. 
3.3. Theorem. For every $T \in \mathrm{CDD}, \mathfrak{b}\left(T^{*}\right)=[\mathfrak{b}(T)]^{*}$.

Proof. Let $T=Q|T|$ be the polar decomposition of $T$. Then $T^{*}=$ $Q^{*}\left|T^{*}\right|$ is the polar decomposition of $T^{*}$. Put $\mathcal{H}=\overline{\mathcal{D}}(T), S=\mathfrak{b}(T)$ and $S^{\prime}=\mathfrak{b}\left(T^{*}\right)$. Fix $x, y \in \mathcal{H}$, put $u=(I+|T|)^{-1} x \in \mathcal{D}(T)$ and $v=\left(I+\left|T^{*}\right|\right)^{-1} y \in \mathcal{D}\left(T^{*}\right)$ and observe that

$$
\begin{gathered}
\langle S x, y\rangle=\left\langle T u,\left(I+\left|T^{*}\right|\right) v\right\rangle=\langle T u, v\rangle+\left\langle Q|T| u,\left|T^{*}\right| v\right\rangle= \\
=\left\langle u, T^{*} v\right\rangle+\left\langle|T| u, T^{*} v\right\rangle=\left\langle(I+|T|) u, T^{*} v\right\rangle=\left\langle x, S^{\prime} y\right\rangle
\end{gathered}
$$

which finishes the proof.

Involving $\mathfrak{b}$-transform we now easily prove

3.4. Theorem. Let $\mathcal{H}$ be a nonseparable Hilbert space and $\left\{T_{s}\right\}_{s \in S} \subset$ $\operatorname{CDD}(\mathcal{H})$ be a countable family of operators. For every nonzero $x \in \mathcal{H}$ there is a separable space $E \subset \mathcal{H}$ containing $x$ such that $E \in \operatorname{red}\left(T_{s}\right)$ for each $s \in S$.

Proof. Thanks to point (D) of Proposition 3.2, we may assume each of $T_{s}$ 's is bounded (because we may replace $T_{s}$ by $\mathfrak{b}\left(T_{s}\right)$ ). Now it suffices to put $E=\varlimsup \operatorname{lin}\left\{S_{1} \ldots S_{n} x \mid n \geqslant 1, S_{1}, \ldots, S_{n} \in\left\{T_{s}: s \in S\right\} \cup\left\{T_{s}^{*}: s \in\right.\right.$ $S\} \cup\{I\}\}$ where $I$ is the identity operator on $\mathcal{H}$.

Now for $\boldsymbol{A}=\left(A_{1}, \ldots, A_{N}\right) \in \mathrm{CDD}_{N}$ put $\mathfrak{b}(\boldsymbol{A})=\left(\mathfrak{b}\left(A_{1}\right), \ldots, \mathfrak{b}\left(A_{N}\right)\right)$ and $\mathfrak{b}(\mathbb{A})=\mathbb{X}$ where $\boldsymbol{X}=\mathfrak{b}(\boldsymbol{A})$. Below we list most important properties of the $\mathfrak{b}$-transform on $\mathcal{C D} \mathcal{D}_{N}$ and $\mathrm{CDD}_{N}$.

(BT1) $\mathfrak{b}(\mathbb{A})=\mathbb{O} \Longleftrightarrow \mathbb{A}=\mathbb{O}$.

(BT2) $\mathfrak{b}(\mathbb{A})$ is bounded, $\mathfrak{b}\left(\mathbb{A}^{*}\right)=[\mathfrak{b}(\mathbb{A})]^{*},|\mathfrak{b}(\mathbb{A})|=\mathfrak{b}(|\mathbb{A}|)$ and $\mathbb{Q}_{\mathfrak{b}(\mathbb{A})}=$ $\mathbb{Q}_{\mathbb{A}}$.

(BT3) $\mathcal{W}^{\prime}(\boldsymbol{A})=\mathcal{W}^{\prime}(\mathfrak{b}(\boldsymbol{A})), \mathcal{W}^{\prime \prime}(\boldsymbol{A})=\mathcal{W}(\mathfrak{b}(\boldsymbol{A})) ; \operatorname{red}(\boldsymbol{A})=\operatorname{red}(\mathfrak{b}(\boldsymbol{A}))$ and $\operatorname{cred}(A)=\operatorname{cred}(\mathfrak{b}(A))$; for every $E \in \operatorname{red}(A), \mathfrak{b}\left(\left.A\right|_{E}\right)=$ $\left.\mathfrak{b}(A)\right|_{E}$.

(BT4) $\mathfrak{b}\left(\bigoplus_{s \in S} \mathbb{A}^{(s)}\right)=\bigoplus_{s \in S} \mathfrak{b}\left(\mathbb{A}^{(s)}\right)$

(BT5) If ' $\sim$ ' denotes one of the relations $=, \leqslant, \leqslant^{s}, \ll, \perp_{u}$, then $\mathbb{A} \sim$ $\mathbb{B} \Longleftrightarrow \mathfrak{b}(\mathbb{A}) \sim \mathfrak{b}(\mathbb{B})$.

\section{Background of von Neumann algebras}

Let $\mathcal{M}$ be a von Neumann subalgebra of $\mathcal{B}(\mathcal{H})$. Denote by $E(\mathcal{M})$ the set of all orthogonal projections in $\mathcal{M}$ and by $\mathcal{Z}(\mathcal{M})$ the center of $\mathcal{M}$. By ' $\sim$ ' we shall denote the Murray-von Neumann equivalence on $E(\mathcal{M})$. Further, put $\mathcal{E}(\mathcal{M})=E(\mathcal{M}) / \sim$ and let ' $\preccurlyeq$ ' denote the Murray-von Neumann order on $\mathcal{E}(\mathcal{M})$. Finally, for each $p \in E(\mathcal{M})$, $c_{p} \in E(\mathcal{Z}(\mathcal{M}))$ stands for the central support of $p$.

It was observed by several mathematicians that the order ' $\leqslant$ ' on $\mathcal{D D}$ translates into the Murray-von Neumann order between (equivalence classes of) projections in a suitable von Neumann algebra. This was explicitly stated and proved in [9, Proposition 1.35]. It is nothing 
new that the same idea works for tuples of operators. We formulate this precisely in the next result which is the main tool of the paper.

4.1. Proposition. Let $\boldsymbol{T} \in \mathrm{CDD}_{N}(\mathcal{H}), E, F \in \operatorname{red}(\boldsymbol{T}), \boldsymbol{A}=\left.\boldsymbol{T}\right|_{E}$ and $\boldsymbol{B}=\left.\boldsymbol{T}\right|_{F}$. Further, let $\mathcal{M}=\mathcal{W}^{\prime}(\boldsymbol{T}), p=P_{E}$ and $q=P_{F}(p, q \in$ $E(\mathcal{M}))$. Then

(a) $A \equiv B \Longleftrightarrow p \sim q$,

(b) $A \leqslant B \Longleftrightarrow p \preccurlyeq q$,

(c) $\boldsymbol{A} \leqslant^{s} B \Longleftrightarrow p \sim c_{p} q$,

(d) $A \perp_{u} B \Longleftrightarrow c_{p} c_{q}=0$,

(e) $\boldsymbol{A} \ll \boldsymbol{B} \Longleftrightarrow p \leqslant c_{q}$.

Proof. We shall only prove (c), since the other points are covered by [9, Proposition 1.35] ((d) is stated there in other form, its recent form may be deduced e.g. from [34, Lemma 1.7]). For this purpose put $\mathcal{M}_{0}=q \mathcal{M} q, z_{0}=c_{p} q \in E\left(\mathcal{Z}\left(\mathcal{M}_{0}\right)\right)$ and let $K \in \operatorname{cred}(B)$ be the range of $z_{0}$. If $z_{0} \sim p$, then by (a), $\left.\boldsymbol{A} \equiv \boldsymbol{B}\right|_{K}$ and thus $\boldsymbol{A} \leqslant^{s} \boldsymbol{B}$. Conversely, if the latter inequality is fulfilled, there is $z_{0} \in E\left(\mathcal{Z}\left(\mathcal{M}_{0}\right)\right)$ such that $p \sim z_{0}$ (again by (a)). But $\mathcal{Z}\left(\mathcal{M}_{0}\right)=\mathcal{Z}(\mathcal{M}) q$ and hence $z_{0}=z q$ for some $z \in E\left(\mathcal{Z}(\mathcal{M})\right.$ ). To this end, note that $c_{p}=c_{z q}$ (since $p \sim z q$ ) and $c_{z q}=z c_{q}$ and therefore $z q=z c_{q} q=c_{p} q$.

Some of consequences of Proposition 4.1 are formulated below (these are adaptations of suitable results of [9]).

(PR1) $\mathbb{A} \sim \mathbb{X} \oplus \mathbb{Y}$ and $\mathbb{A} \perp_{u} \mathbb{Y}$ imply $\mathbb{A} \sim \mathbb{X}$ when ' $\sim$ ' is replaced by one of $\leqslant, \leqslant^{s}, \ll$.

(PR2) If $\mathbb{A}^{(s)} \perp_{u} \mathbb{B}^{(t)}$ for all $s \in S$ and $t \in T$, then $\bigoplus_{s \in S} \mathbb{A}^{(s)} \perp_{u}$ $\bigoplus_{t \in T} \mathbb{B}^{(t)}$.

(PR3) A function $\operatorname{cred}(\boldsymbol{A}) \ni E \mapsto \mathbb{X}(E) \in\left\{\mathbb{B} \in \mathcal{C D D D}_{N}: \mathbb{B} \leqslant \leqslant^{s} \mathbb{A}\right\}$ where $X(E)=\left.A\right|_{E}$ is a (well defined) bijection.

(PR4) For every $E \in \operatorname{red}(\boldsymbol{A}),\left.\left.\boldsymbol{A}\right|_{E} \perp_{u} A\right|_{E^{\perp}} \Longleftrightarrow E \in \operatorname{cred}(\boldsymbol{A})$.

(PR5) For every pair $(\mathbb{A}, \mathbb{B})$ such that $\mathbb{A} \leqslant^{s} \mathbb{B}$ there is a unique $\mathbb{X} \in$ $\mathrm{CDD}_{N}$ such that $\mathbb{B}=\mathbb{A} \boxplus \mathbb{X}$. Notation: $\mathbb{B} \boxminus \mathbb{A}:=\mathbb{X}$. (So, $\mathbb{B} \boxminus \mathbb{A}$ makes sense iff $\mathbb{A} \leqslant \leqslant^{s} \mathbb{B}$.)

(PR6) For every $\mathbb{X} \in \mathcal{C} \mathcal{D D}_{N}$ and a cardinal $\alpha,\left\{\mathbb{Y} \in \mathcal{C D D}_{N}: \mathbb{Y} \leqslant \leqslant^{s}\right.$ $\alpha \odot \mathbb{X}\}=\left\{\alpha \odot \mathbb{Y}: \mathbb{Y} \leqslant{ }^{s} \mathbb{X}\right\}$.

Following (PR5), let us agree with the following convention: whenever for a pair $(\mathbb{A}, \mathbb{B})$ there is a unique $\mathbb{X}$ for which $\mathbb{B}=\mathbb{A} \oplus \mathbb{X}$, we shall denote this unique $\mathbb{X}$ by $\mathbb{B} \ominus \mathbb{A}$. Observe that $\mathbb{A} \leqslant \mathbb{B}$ provided $\mathbb{B} \ominus \mathbb{A}$ makes sense.

Combining Proposition 4.1 with Sherman's theorem [31], we obtain an interesting

4.2. Theorem. $\left(\mathcal{C D} \mathcal{D}_{N}, \leqslant\right)$ is an order-complete lattice. Precisely, for every nonempty family (i.e. a set) $\left\{\mathbb{A}^{(s)}\right\}_{s \in S} \subset \mathcal{C D} \mathcal{D}_{N}$ there are members $\mathbb{X}$ and $\mathbb{Y}$ of $\mathcal{C D D}_{N}$ such that $\mathbb{X} \leqslant \mathbb{A}^{(s)} \leqslant \mathbb{Y}$ for each $s \in S$ 
and $\mathbb{X}^{\prime} \leqslant \mathbb{X}$ (respectively $\mathbb{Y} \leqslant \mathbb{Y}^{\prime}$ ) whenever $\mathbb{X}^{\prime} \leqslant \mathbb{A}^{(s)}$ (respectively $\left.\mathbb{A}^{(s)} \leqslant \mathbb{Y}^{\prime}\right)$ for all $s \in S$.

Proof. Put $\mathbb{A}=\bigoplus_{s \in S} \mathbb{A}^{(s)}$ and $\mathcal{M}=\mathcal{W}^{\prime}(A)$. By [31], $(\mathcal{E}(\mathcal{M}), \preccurlyeq)$ is an order-complete lattice. So, using Proposition 4.1 we see that there are $\mathbb{X}$ and $\mathbb{Y}$ (both less than or equal to $\mathbb{A}$ ) which correspond to the g.l.b. and l.u.b. (with respect to ' $\preccurlyeq$ ') of the projections corresponding to $\mathbb{A}^{(s)}$ 's. Now if $\mathbb{X}^{\prime}$ and $\mathbb{Y}^{\prime}$ are as in the statement of the theorem, consider $\widetilde{\mathbb{A}}=\mathbb{A} \oplus \mathbb{X}^{\prime} \oplus \mathbb{Y}^{\prime}$ and $\widetilde{\mathcal{M}}=\mathcal{W}^{\prime}(\widetilde{\boldsymbol{A}})$ and repeat the above argument to get the assertion. We skip the details.

As it is usually done when working with lattices, for every nonempty collection $\mathcal{A}=\left\{\mathbb{A}^{(s)}\right\}_{s \in S}$ we shall denote by $\bigvee_{s \in S} \mathbb{A}^{(s)}$ and $\bigwedge_{s \in S} \mathbb{A}^{(s)}$ the l.u.b. and g.l.b of $\mathcal{A}$. Observe that $\mathbb{A} \perp_{u} \mathbb{B}$ iff $\mathbb{A} \wedge \mathbb{B}=\mathbb{O}$.

\section{Decompositions Relative to ideAls}

Let $\mathcal{A}$ be a subclass of $\mathrm{CDD}_{N}$. We call $\mathcal{A}$ an ideal iff $\mathcal{A}$ satisfies the following four conditions:

(ID1) $\mathcal{A}$ is nonempty,

(ID2) whenever $\boldsymbol{A} \in \mathcal{A}$ and $\boldsymbol{A} \equiv \boldsymbol{B} \in \mathrm{CDD}_{N}$, then $\boldsymbol{B} \in \mathcal{A}$,

(ID3) for every $\boldsymbol{A} \in \mathcal{A}$ and $E \in \operatorname{red}(\boldsymbol{A}),\left.\boldsymbol{A}\right|_{E} \in \mathcal{A}$,

(ID4) $\bigoplus_{s \in S} \boldsymbol{A}_{s} \in \mathcal{A}$ for any nonempty family $\left\{\boldsymbol{A}_{s}\right\}_{s \in S} \subset \mathcal{A}$.

Classical examples of ideals the reader may find in Examples 5.3 below.

For every subclass $\mathcal{F}$ of $\mathrm{CDD}_{N}$ put

$$
\mathcal{F}^{\perp}=\left\{\boldsymbol{T} \in \mathrm{CDD}_{N}: \boldsymbol{T} \perp_{u} \boldsymbol{F} \text { for every } \boldsymbol{F} \in \mathcal{F}\right\} .
$$

It is easily seen that $\mathcal{F}^{\perp}$ is an ideal for any $\mathcal{F} \subset \mathrm{CDD}_{N}$ (thanks to (PR2)). As we will see later, the 'converse' is also true, that is, $\mathcal{A}$ is an ideal iff $\mathcal{A}=\left(\mathcal{A}^{\perp}\right)^{\perp}$. This reminds anologous characterization of closed linear subspaces of Hilbert spaces. However, the just defined 'orthogonal complement' is more familiar to the orthogonality in spaces of measures than to that in Hilbert spaces.

One of main results of the paper is the following

5.1. Theorem. Let $\mathcal{A} \subset \mathrm{CDD}_{N}$ be an ideal. For every $\boldsymbol{T} \in \mathrm{CDD}_{N}$ there is a unique $E \in \operatorname{red}(\boldsymbol{T})$ such that

$$
\left.T\right|_{E} \in \mathcal{A} \quad \text { and }\left.\quad T\right|_{E^{\perp}} \in \mathcal{A}^{\perp} .
$$

Moreover, $E \in \operatorname{cred}(T)$ and

$$
E=\bigvee\left\{K \in \operatorname{red}(\boldsymbol{T}):\left.T\right|_{K} \in \mathcal{A}\right\} .
$$

Proof. First we shall show the existence of $E$. We may assume that $\boldsymbol{T} \notin$ $\mathcal{A}^{\perp}$. By Zorn's lemma, there is a maximal family $\left\{E_{s}\right\}_{s \in S}$ of mutually orthogonal nontrivial reducing (for $T$ ) subspaces with $\left.T\right|_{E_{s}} \in \mathcal{A}$ for every $s \in S$. It is clear that (5-1) is satisfied with $E=\bigvee_{s \in S} E_{s}$. 
Now assume that $E \in \operatorname{red}(\boldsymbol{T})$ is as in (5-1). By (PR4), $E \in \operatorname{cred}(\boldsymbol{T})$. To establish uniqueness and finish the proof it is enough to check (5-2). But this simply follows from (PR1) and Proposition 4.1. (Indeed, if $K \in \operatorname{red}(\boldsymbol{T})$ is such that $\left.\boldsymbol{T}\right|_{K} \in \mathcal{A}$, then $\left.\boldsymbol{T}\right|_{K} \leqslant\left.\left.\boldsymbol{T}\right|_{E} \oplus \boldsymbol{T}\right|_{E^{\perp}}$ and $\left.\left.\boldsymbol{T}\right|_{K} \perp_{u} \boldsymbol{T}\right|_{E^{\perp}}$. So, we conclude from (PR1) that $\left.\boldsymbol{T}\right|_{K} \leqslant\left.\boldsymbol{T}\right|_{E}$. Thus, by Proposition 4.1, $P_{K} \preccurlyeq P_{E}$ in $\mathcal{M}=\mathcal{W}^{\prime}(\boldsymbol{T})$. But $P_{E} \in \mathcal{Z}(\mathcal{M})$ and hence $P_{K} \leqslant P_{E}$ which means that $K \subset E$.)

For simplicity, let us introduce the following notation. For every ideal $\mathcal{A} \subset \mathrm{CDD}_{N}, \mathcal{A}^{(0)}=\mathcal{A}$ and $\mathcal{A}^{(1)}=\mathcal{A}^{\perp}$. Under such a notation, by a simple induction argument we obtain

5.2. Theorem. Let $\mathcal{A}_{1}, \ldots, \mathcal{A}_{k} \subset \mathrm{CDD}_{N}$ be ideals. For every $\boldsymbol{T} \in$ $\mathrm{CDD}_{N}(\mathcal{H})$ there is a unique system $\left\{E_{\delta}\right\}_{\delta \in\{0,1\}^{k}}$ of reducing subspaces for $\boldsymbol{T}$ such that

(i) $E_{\delta} \perp E_{\delta^{\prime}}$ for distinct $\delta, \delta^{\prime} \in\{0,1\}^{k}$; and $\mathcal{H}=\bigoplus_{\delta \in\{0,1\}^{k}} E_{\delta}$,

(ii) $\left.T\right|_{E_{\delta}} \in \bigcap_{j=1}^{k} \mathcal{A}_{j}^{\left(\delta_{j}\right)}$ for each $\delta \in\{0,1\}^{k}$.

Moreover, $E_{\delta} \in \operatorname{cred}(\boldsymbol{T})$ for every $\delta \in\{0,1\}^{k}$.

We leave the proof of Theorem 5.2 for the reader.

Theorem 5.2 covers any known result on decomposition of a single operator into two parts with first of them of a special class and the other 'completely' (or 'hereditarily') not of this class. Examples to this are given below.

5.3. Examples. (A) Let $F$ be a closed subset of the complex plane $\mathbb{C}$. Let $\mathcal{N}(F)$ be the class of all normal operators whose spectrum is contained in $F$. (Here we assume that operators on zerodimensional Hilbert spaces are normal and have empty spectra.) It is easily checked that $\mathcal{N}(F)$ is an ideal. Thus, every operator $T \in \mathrm{CDD}$ admits a unique decomposition into a part in $\mathcal{N}(F)$ and the remainder in $\mathcal{N}(F)^{\perp}$. This means that there is a unique $E \in \operatorname{red}(T)$ such that $\left.T\right|_{E}$ is normal, $\sigma\left(\left.T\right|_{E}\right) \subset F$ and $\left.T\right|_{E^{\perp}}$ has no nontrivial reduced part which belongs to $\mathcal{N}(F)$. When $F=\mathbb{C}$, this is the decomposition into the normal part and the completely non-normal one. When $F=\mathbb{R}$, we get the decomposition into the selfadjoint part and the completely non-selfadjoint one. Finally, when $F=\{z \in \mathbb{C}:|z|=1\}$, the operator decomposes into the unitary part and the completely non-unitary one. These three cases are most classical. (Compare with [9], page 179.)

(B) Single operators of each of the following classes form an ideal: formally normal (for definition see e.g. [33]); quasinormal; hyponormal; subnormal; contractions. As we will see in Proposition 5.4, also the following class $\mathcal{A}$ is an ideal: $T \in \mathcal{A}$ iff $T$ is the direct sum of bounded operators. 
(C) Stochel and Szafraniec [33] have shown that every operator $T \in$ CDD admits a unique decomposition of the form $T=T_{n o r} \oplus T_{p f n} \oplus$ $T_{c n f n}$ where $T_{n o r}$ is normal, $T_{p f n}$ is purely formally normal (here 'purely' means that $T_{p f n}$ is in addition completely non-normal) and $T_{c n f n}$ is completely non-formally normal. Their result is a special case of Theorem 5.2.

(D) Ernest [9] distinguishes an important class of bounded operators on separable Hilbert spaces, the so-called smooth operators (see $\S 6$ of Chapter 1 in [9]). Let us say that an operator $T \in \operatorname{CDD}(\mathcal{H})$ where $\mathcal{H}$ is separable is $\sigma$-smooth iff $\mathfrak{b}(T)$ is the direct sum of countably (finitely or infinitely) many smooth operators. By Proposition 1.52 of [9] and Proposition 5.4 below, operators which are direct sums of $\sigma$-smooth operators form an ideal. In particular, every closed densely defined operator acting on a separable Hilbert space admits a unique decomposition into a $\sigma$-smooth operator and a completely non-smooth one.

(E) Let us give some examples dealing with systems of operators. Let $\mathcal{N}_{N}$ and $\widetilde{\mathcal{N}}_{N}$ consist of all $N$-tuples (belonging to $\mathrm{CDD}_{N}$ ) of, respectively, commuting normal and arbitrary normal operators (commutativity may be defined by means of the spectral measures or, equivalently, of the $\mathfrak{b}$-transforms). It is clear that both $\mathcal{N}_{N}$ and $\widetilde{\mathcal{N}}_{N}$ are ideals. So, every $\boldsymbol{T} \in \mathrm{CDD}_{N}$ has a unique decomposition in the form $\boldsymbol{T}=\boldsymbol{T}_{j n} \oplus \boldsymbol{T}_{p s n} \oplus \boldsymbol{T}_{c n s n}$ where $\boldsymbol{T}_{j n} \in \mathcal{N}_{N}, \boldsymbol{T}_{p s n} \in \widetilde{\mathcal{N}}_{N}$ and no nontrivial reduced part of $\boldsymbol{T}_{p s n}$ is a member of $\mathcal{N}_{N}$, and no nontrivial reduced part of $\boldsymbol{T}_{\text {cnsn }}$ belongs to $\widetilde{\mathcal{N}}_{N}$. (The labels 'jn', 'psn' and 'cnsn' appearing here are the abbreviations for jointly normal, purely separately normal and completely non-separately normal.) We call an $N$-tuple $\mathbb{A}$ normal iff $\boldsymbol{A} \in \mathcal{N}_{N}$.

(F) If $\mathcal{A} \subset \mathrm{CDD}$ is an ideal, so are $\Delta_{N}(\mathcal{A}) \subset \mathrm{CDD}_{N}$ and $\mathcal{A}^{[N]} \subset \mathrm{CDD}_{N}$ where $\mathcal{A}^{[N]}$ consists of all $N$-tuples $\left(A_{1}, \ldots, A_{N}\right)$ with $A_{1}, \ldots, A_{N} \in$ $\mathcal{A}$ acting in a common Hilbert space, and

$$
\Delta_{N}(\mathcal{A})=\left\{\left(A_{1}, \ldots, A_{N}\right): A_{1}=\ldots=A_{N} \in \mathcal{A}\right\} .
$$

(G) Theorem 5.1 may be shortly reformulated in the following significant way: $\mathrm{CDD}_{N}=\mathcal{A} \oplus \mathcal{A}^{\perp}$ for every ideal $\mathcal{A} \subset \mathrm{CDD}_{N}$. Using this notation, Theorem 5.2 with $k=2$ asserts that

$$
\mathrm{CDD}_{N}=(\mathcal{A} \cap \mathcal{B}) \oplus\left(\mathcal{A} \cap \mathcal{B}^{\perp}\right) \oplus\left(\mathcal{A}^{\perp} \cap \mathcal{B}\right) \oplus\left(\mathcal{A}^{\perp} \cap \mathcal{B}^{\perp}\right)
$$

for any two ideals $\mathcal{A}$ and $\mathcal{B}$ in $\mathrm{CDD}_{N}$. The counterpart of (5-3) for linear subspaces $K$ and $L$ of a Hilbert space $\mathcal{H}$ is fulfilled only when $P_{K}$ and $P_{L}$ commute. Thus, as we have said earlier, the 'orthogonal complement' for ideals behaves in a similar manner as the orthogonal complement of lattices of measures (or of more general structures such as abstract $L$-spaces).

The next result is useful for producing ideals. 
5.4. Proposition. Let $\mathcal{A}$ be a subclass of $\mathrm{CDD}_{N}$ and $\Theta_{N}$ be the class of all trivial members of $\mathrm{CDD}_{N}$.

(a) The class

$$
\begin{aligned}
& J(\mathcal{A})=\left\{\boldsymbol{T} \in \mathrm{CDD}_{N}: \text { for some set } S, \boldsymbol{T}=\bigoplus_{s \in S} \boldsymbol{X}^{(s)}\right. \\
& \text { with } \left.\boldsymbol{X}^{(s)} \leqslant \boldsymbol{Y}^{(s)} \in \mathcal{A} \cup \Theta_{N}\right\}
\end{aligned}
$$

is an ideal and it is the smallest ideal which contains $\mathcal{A}$.

(b) $\mathcal{A}$ is an ideal iff $\mathcal{A}=\left(\mathcal{A}^{\perp}\right)^{\perp}$.

Proof. To show (a), we only need to check that $A \in J(\mathcal{A})$ provided $\boldsymbol{A} \leqslant \bigoplus_{s \in S} \boldsymbol{Y}^{(s)}$ with $\boldsymbol{Y}^{(s)} \in \mathcal{A}$. Assuming $\boldsymbol{A}$ is nontrivial, take a maximal family $\mathcal{E}=\left\{E_{\gamma}\right\}_{\gamma \in \Gamma}$ of mutually orthogonal nontrivial reducing subspaces for $\boldsymbol{A}$ such that $\left.\boldsymbol{A}\right|_{E_{\gamma}} \leqslant \boldsymbol{X}^{(\gamma)}$ for some $\boldsymbol{X}^{(\gamma)} \in \mathcal{A}(\gamma \in \Gamma)$. Let $F$ be the orthogonal complement of $\bigoplus_{\gamma \in \Gamma} E_{\gamma}$ (in $\overline{\mathcal{D}}(\boldsymbol{A})$ ). We only need to check that $F$ is trivial. We infer from the maximality of $\mathcal{E}$ that $\left.\boldsymbol{A}\right|_{F} \in \mathcal{A}^{\perp}$. Thus, thanks to (PR2), $\left.\boldsymbol{A}\right|_{F} \perp_{u} \bigoplus_{s \in S} \boldsymbol{Y}^{(s)}$ and hence, by (PR1), $\left.\boldsymbol{A}\right|_{F}$ is trivial and we are done.

The 'if' part of (b) is immediate, while the 'only if' one follows from Theorem 5.1.

5.5. Remark. In Proposition 10.1 (page 25) we shall show that for every ideal $\mathcal{A}$ there is a (unique up to unitary equivalence under some additional properties of $\boldsymbol{A}) N$-tuple $\boldsymbol{A}$ such that $\mathcal{A}=\{\boldsymbol{B}: \boldsymbol{B} \ll \boldsymbol{A}\}$. Thus, our Theorem 5.1 is a generalization of Ernest's Proposition 2.12 in [9].

The rest of the paper is devoted to the class $\mathcal{C D} \mathcal{D}_{N}$.

\section{ORDER ' $\leqslant$,}

Everywhere below the prefix ' $\leqslant$ ' says that the suitable term is understood with respect to this order. The aim of this part is to prove

6.1. Theorem. Let $\mathfrak{B}$ be a nonempty set of members of $\mathcal{C D D}_{N}$ and let $\mathbb{A}, \mathbb{B} \in \mathcal{C D D}_{N}$.

(A) $\mathfrak{B}$ has the $\leqslant^{s}-$ g.l.b.

(B) $\mathfrak{B}$ has the $\leqslant^{s}$-l.u.b. if and only if every two-point subset of $\mathfrak{B}$ is $\leqslant^{s}$-upper bounded. If the latter happens, inf $\leqslant_{s} \mathfrak{B}=\bigwedge \mathfrak{B}$ and $\sup _{\leqslant s} \mathfrak{B}=\bigvee \mathfrak{B}$.

(C) The following conditions are equivalent:

(i) the set $\{\mathbb{A}, \mathbb{B}\}$ is $\leqslant^{s}$-upper bounded,

(ii) $\mathbb{A} \leqslant^{s} \mathbb{A} \vee \mathbb{B}$ and $\mathbb{B} \leqslant^{s} \mathbb{A} \vee \mathbb{B}$,

(iii) $\mathbb{A}$ and $\mathbb{B}$ may be written in the forms $\mathbb{A}=\mathbb{E} \boxplus \mathbb{X}$ and $\mathbb{B}=\mathbb{E} \boxplus \mathbb{Y}$ for some $\mathbb{E}, \mathbb{X}, \mathbb{Y} \in \mathcal{C D D}_{N}$ such that $\mathbb{X} \perp_{u} \mathbb{Y}$.

(D) If $\{\mathbb{A}, \mathbb{B}\}$ is $\leqslant^{s}$-upper bounded, then $\mathbb{A} \leqslant \mathbb{B} \Longleftrightarrow \mathbb{A} \leqslant$. 
Proof. We begin with $(\mathrm{C})$. Implications (iii) $\Longrightarrow($ ii $) \Longrightarrow$ (i) are immediate (indeed, if (iii) is fulfilled, $\mathbb{A} \vee \mathbb{B}=\mathbb{E} \boxplus \mathbb{X} \boxplus \mathbb{Y}$ ). To see that (iii) follows from (i), let $\mathbb{F} \in \mathcal{C D D}_{N} \leqslant^{s}$-majorizes $\mathbb{A}$ and $\mathbb{B}$. This means that $\left.\boldsymbol{A} \equiv \boldsymbol{F}\right|_{K}$ and $\left.\boldsymbol{B} \equiv \boldsymbol{F}\right|_{L}$ for some $K, L \in \operatorname{cred}(\boldsymbol{F})$. Then $P_{K}$ and $P_{L}$ commute and therefore $K=M \oplus K^{\prime}$ and $L=M \oplus L^{\prime}$ where $M=K \cap L, K^{\prime}=M^{\perp} \cap K$ and $L^{\prime}=M^{\perp} \cap L$. Note that then $\boldsymbol{E}=\left.\boldsymbol{F}\right|_{M}$, $\boldsymbol{X}=\left.\boldsymbol{F}\right|_{K^{\prime}}$ and $\boldsymbol{Y}=\left.\boldsymbol{F}\right|_{L^{\prime}}$ are pairwise unitarily disjoint and $\mathbb{A}=\mathbb{E} \boxplus \mathbb{X}$ and $\mathbb{B}=\mathbb{E} \boxplus \mathbb{Y}$.

Now we pass to (B). Suppose every two-point subset of $\mathfrak{B}$ is $\leqslant^{s}$ upper bounded. Let $\mathbb{M}$ be such that $\mathbb{B} \leqslant \mathbb{M}$ for every $\mathbb{B} \in \mathfrak{B}$. Put $\mathcal{M}=\mathcal{W}^{\prime}(\boldsymbol{M})$. For every $\mathbb{B} \in \mathfrak{B}$ take $K(\mathbb{B}) \in \operatorname{red}(\boldsymbol{M})$ such that $\left.\boldsymbol{B} \equiv \boldsymbol{M}\right|_{K(\mathbb{B})}$ and put $p_{\mathbb{B}}=P_{K(\mathbb{B})} \in \mathcal{M}$.

For a moment fix $\mathbb{A}, \mathbb{B} \in \mathfrak{B}$. By $(\mathrm{C})$, there is $\mathbb{F} \leqslant \mathbb{M}$ such that $\mathbb{A} \leqslant s$ and $\mathbb{B} \leqslant^{s} \mathbb{F}$. We infer from this, involving Proposition 4.1, that there is a projection $q \in E(\mathcal{M})$ for which $p_{\mathbb{A}} \sim c_{p_{\mathbb{A}}} q$ and $p_{\mathbb{B}} \sim c_{p_{\mathbb{B}}} q$. Notice that then $c_{p_{\mathbb{B}}} p_{\mathbb{A}} \sim c_{p_{\mathbb{B}}} c_{p_{\mathbb{A}}} q$ and $c_{p_{\mathbb{A}}} p_{\mathbb{B}} \sim c_{p_{\mathbb{A}}} c_{p_{\mathbb{B}}} q$. This proves that

$$
c_{p_{\mathbb{B}}} p_{\mathbb{A}} \sim c_{p_{\mathbb{A}}} p_{\mathbb{B}}
$$

for all $\mathbb{A}, \mathbb{B} \in \mathfrak{B}$. Now put $w=\bigvee\left\{c_{p_{\mathbb{A}}}: \mathbb{A} \in \mathfrak{B}\right\} \in \mathcal{Z}(\mathcal{M})$. There is a family $\left\{z_{\mathbb{A}}\right\}_{\mathbb{A} \in \mathfrak{B}}$ of mutually orthogonal central projections in $\mathcal{M}$ such that $z_{\mathbb{A}} \leqslant c_{p_{\mathbb{A}}}$ for every $\mathbb{A} \in \mathfrak{B}$ and $\sum_{\mathbb{A} \in \mathfrak{B}} z_{\mathbb{A}}=w$. Put

$$
q=\sum_{\mathbb{A} \in \mathfrak{B}} z_{\mathbb{A}} p_{\mathbb{A}} \in E(\mathcal{M}) .
$$

For $\mathbb{A}, \mathbb{B} \in \mathfrak{B}$ we have, by $(6-1), z_{\mathbb{B}} c_{p_{\mathbb{A}}} q=z_{\mathbb{B}} c_{p_{\mathbb{A}}} p_{\mathbb{B}} \sim z_{\mathbb{B}} c_{p_{\mathbb{B}}} p_{\mathbb{A}}=z_{\mathbb{B}} p_{\mathbb{A}}$ and consequently ( since $w \geqslant c_{p_{\mathbb{A}}}$ ),

$$
p_{\mathbb{A}}=\sum_{\mathbb{B} \in \mathfrak{B}} z_{\mathbb{B}} p_{\mathbb{A}} \sim \sum_{\mathbb{B} \in \mathfrak{B}} z_{\mathbb{B}} c_{p_{\mathbb{A}}} q=c_{p_{\mathbb{A}}} q .
$$

Now if $E \in \operatorname{red}(\boldsymbol{M})$ is the range of $q$ and $\boldsymbol{M}^{\prime}=\left.\boldsymbol{M}\right|_{E}$, Proposition 4.1 shows that $\mathbb{A} \leqslant s \mathbb{M}^{\prime}$ for every $\mathbb{A} \in \mathfrak{B}$. Hence, replacing $\mathbb{M}$ by $\mathbb{M}^{\prime}$, we may assume that $p_{\mathbb{A}} \in \mathcal{Z}(\mathcal{M})$. It is known that in such a case $\bigvee_{\mathbb{A} \in \mathfrak{B}} p_{\mathbb{A}}$ and $\bigwedge_{\mathbb{A} \in \mathfrak{B}} p_{\mathbb{A}}$ are, respectively, the l.u.b. and the g.l.b. with respect to ' $\preccurlyeq$ ' in $E(\mathcal{M})$. It is left as an exercise that the assertion of $(\mathrm{B})$ now follows.

Finally, (A) is implied by (B), and (D) is left for the reader.

As a very special case of Theorem 6.1 we get

6.2. Corollary. If $\left\{\mathbb{A}^{(s)}\right\}_{s \in S}$ is a nonempty family of mutually unitarily disjoint $N$-tuples, then $\bigvee_{s \in S} \mathbb{A}^{(s)}=\boxplus_{s \in S} \mathbb{A}^{(s)}$.

Proof. One easily checks that $\boxplus_{s \in S} \mathbb{A}^{(s)}$ is the $\leqslant^{s}$-l.u.b. of $\left\{\mathbb{A}^{(s)}\right\}_{s \in S}$. Thus the assertion follows from Theorem 6.1.

6.3. Example. $[N=1]$ Let $I_{j}$ for $j=1,2$ be the identity operator on a $j$-dimensional Hilbert space. It is clear that $\mathbb{I}_{1} \leqslant \mathbb{I}_{2}, \mathbb{I}_{1} \wedge \mathbb{I}_{2}=\mathbb{I}_{1}$ 
and $\mathbb{I}_{1} \vee \mathbb{I}_{2}=\mathbb{I}_{2}$, while inf $\leqslant_{s}\left\{\mathbb{I}_{1}, \mathbb{I}_{2}\right\}=\mathbb{O}$ and $\left\{\mathbb{I}_{1}, \mathbb{I}_{2}\right\}$ is not $\leqslant^{s}$-upper bounded. This shows that the $\leqslant^{s}$-g.l.b. in general differs from the $\leqslant-$-g.l.b. (although both of them always exist).

We end the section with a useful

6.4. Proposition. (A) If $\mathbb{A} \leqslant \boxplus_{s \in S} \mathbb{B}^{(s)}$, then $\mathbb{A}=\boxplus_{s \in S}\left(\mathbb{A} \wedge \mathbb{B}^{(s)}\right)$.

(B) Suppose $\mathbb{A}^{(s)} \leqslant \mathbb{X}(s \in S \neq \varnothing)$ and $\mathbb{B} \leqslant s \mathbb{X}$. Then

$$
\left[\bigvee_{s \in S} \mathbb{A}^{(s)}\right] \wedge \mathbb{B}=\bigvee_{s \in S}\left[\mathbb{A}^{(s)} \wedge \mathbb{B}\right]
$$

If in addition $\bigoplus_{s \in S} \mathbb{A}^{(s)} \leqslant \mathbb{X}$, then

$$
\left[\bigoplus_{s \in S} \mathbb{A}^{(s)}\right] \wedge \mathbb{B}=\bigoplus_{s \in S}\left[\mathbb{A}^{(s)} \wedge \mathbb{B}\right]
$$

Proof. To prove $(\mathrm{A})$, put $\mathbb{B}=\bigoplus_{s \in S} \mathbb{B}^{(s)}$. Since each of $\mathbb{B}^{(s)}$ 's corresponds to a central projection in $\mathcal{W}^{\prime}(B)$, the assertion easily follows. The same argument works in $(\mathrm{B})$ - here $\mathbb{B}$ corresponds to a central projection in $\mathcal{W}^{\prime}(\boldsymbol{X})$.

A counterpart of a part of Proposition 6.4 for the order ' $\leqslant$ ' will be proved in Theorem 15.10 (page 53). However, this will be much more complicated.

\section{SteERING PROJECTIONS IN $\mathcal{W}^{*}$-ALGEBRAS}

We would like to propose a little bit new approach to the so-called dimension theory of $\mathcal{W}^{*}$-algebras (see e.g. [18, Chapter 5, §5] and [19, Chapter 6]; [34, Chapter 5, §1]; [13, 14]; [36]; [31]). Usually one decomposes a projection in a $\mathcal{W}^{*}$-algebra into (in a sense) 'homogenous' parts, as it was done by Griffin [13, 14], Tomiyama [36] and Sherman [31]. In the next section we will do essentially the same but in a different manner, convenient for applications to the class $\mathcal{C D} \mathcal{D}_{N}$. In every $\mathcal{W}^{*}$-algebra $\mathcal{M}$ we shall distinguish a projection, called steering, and next we shall show how this projection 'controls' the Murray-von Neumann order on $\mathcal{E}(\mathcal{M})$. As we will see, the steering projection is defined in different ways for type $\mathrm{II}_{1}$; type $\mathrm{II}_{\infty}$; and type $\mathrm{I}$ or III algebras. Therefore we shall distinguish our investigations into these three cases.

Type $\mathbf{I I}_{1}$. When $\mathcal{M}$ is a type $\mathrm{II}_{1} \mathcal{W}^{*}$-algebra, it seems to be most appropriate to call the unit of $\mathcal{M}$ the steering projection.

Types I and III. We assume that $\mathcal{M}$ is a type I or III $\mathcal{W}^{*}$-algebra. We say $\mathcal{M}$ is quasi-commutative iff $p \sim c_{p}$ for every $p \in E(\mathcal{M})$. A projection $p \in E(\mathcal{M})$ is quasi-abelian iff $p=0$ or $p \mathcal{M} p$ is quasicommutative.

7.1. Lemma. For $p \in E(\mathcal{M})$ the following conditions are equivalent:

(i) $p$ is quasi-abelian,

(ii) for every $q \in E(\mathcal{M})$ with $q \leqslant p, q \sim c_{q} p$, 
(iii) for every $q \in E(\mathcal{M}), p \preccurlyeq q \Longleftrightarrow p \leqslant c_{q}$.

Proof. The equivalence of (i) and (ii) follows from the fact that the central support of $q \in E(p \mathcal{M} p)$ with respect to $p \mathcal{M} p$ coincides with $c_{q} p$ (where $c_{q}$ is the central support of $q$ in $\mathcal{M}$ ).

To show that (iii) follows from (ii), assume that $0 \neq p \leqslant c_{q}$ and take a maximal family $\left\{p_{s}\right\}_{s \in S} \subset E(\mathcal{M})$ of nonzero projections such that $p_{s} \leqslant p, p_{s} \preccurlyeq q$ for $s \in S$ and $c_{p_{s}} c_{p_{t}} p=0$ for distinct $s, t \in S$. Notice that then $p=\sum_{s \in S} c_{p_{s}} p$ and $c_{p_{s}} c_{p_{t}} c_{p}=0$ for different $s, t \in S$. Now we infer from (ii) that $c_{p_{s}} p \preccurlyeq q$ and consequently $c_{p_{s}} p \preccurlyeq c_{p_{s}} c_{p} q$. So, $p=\sum_{s \in S} c_{p_{s}} p \preccurlyeq\left(\sum_{s \in S} c_{p_{s}} c_{p}\right) q \leqslant q$.

Finally, under the assumption of (iii), for $q \leqslant p$ put $r=q+\left(1-c_{q}\right) p$, notice that $c_{r}=c_{p} \geqslant p$ and thus, by (iii), $p \preccurlyeq r$. Consequently, $c_{q} p \preccurlyeq c_{q} r=q$ and we are done.

A steering projection in (a type I or III $\mathcal{W}^{*}$-algebra) $\mathcal{M}$ is a quasiabelian projection $p \in E(\mathcal{M})$ such that $c_{p}=1$.

7.2. Theorem. (I) Suppose $\mathcal{M}$ is type I. A projection $p \in E(\mathcal{M})$ with $c_{p}=1$ is steering iff $p$ is abelian. In particular, $\mathcal{M}$ has a steering projection and any two steering projections are Murrayvon Neumann equivalent.

(II) Suppose $\mathcal{M}$ is type III. $\mathcal{M}$ has a steering projection and any two steering projections are Murray-von Neumann equivalent.

Proof. Point (I) is left for the reader. We shall give a sketch of proof of (II). If $p$ and $q$ are steering, then $c_{p}=c_{q}=1$ and thus $p \preccurlyeq q$ and $q \preccurlyeq p$, by Lemma 7.1. This establishes uniqueness up to Murray-von Neumann equivalence. To show the existence, take a maximal family $\left\{p_{s}\right\}_{s \in S} \subset E(\mathcal{M})$ of mutually centrally orthogonal nonzero projections each of which is countably decomposable and put $p=\sum_{s \in S} p_{s}$. Such a projection is steering since each of $p_{s}$ 's is quasi-abelian, e.g. by [19, Corollary 6.3.5].

Type $\mathbf{I I}_{\infty}$. Finally, assume $\mathcal{M}$ is a type $\mathrm{II}_{\infty} \mathcal{W}^{*}$-algebra. Since the unit of $\mathcal{M}$ may be written in the form $\sum_{n=1}^{\infty} p_{n}$ with $p_{n} \sim 1$ for each $n \geqslant 1$, for every projection $q \in E(\mathcal{M})$ there is a countable infinite family of mutually orthogonal projections each of which is Murray-von Neumann equivalent to $q$. For each $n \in\{1,2,3, \ldots\} \cup\{\omega\}$ we shall write $n \odot q$ to denote any projection (or, a unique member of $\mathcal{E}(\mathcal{M})$ ) in $\mathcal{M}$ which is the sum of (exactly) $n$ copies of $q$. (Here by a copy we mean any projection which is Murray-von Neumann equivalent to $q$; $\omega \odot q$ is the sum of $\aleph_{0}$ copies of $q$.)

We begin with

7.3. Lemma. For $p \in E(\mathcal{M})$ the following conditions are equivalent:

(i) $p$ is finite, 
(ii) whenever $p \leqslant c_{q}$ for $q \in E(\mathcal{M})$, there is a sequence $\left(z_{n}\right)_{n=1}^{\infty}$ of central projections in $\mathcal{M}$ such that $\sum_{n=1}^{\infty} z_{n}=1$ and $z_{n} p \preccurlyeq n \odot q$ for any $n \geqslant 1$.

Proof. Let $q_{0} \in E(\mathcal{M})$ be a finite projection such that $c_{q_{0}}=1$. If (iii) is satisfied, then $z_{n} p \preccurlyeq n \odot q_{0}$ for a suitable sequence $\left(z_{n}\right)_{n=1}^{\infty}$ of central projections. Then $z_{n} p$ is finite and thus so is $\left(\bigvee_{n \geqslant 1} z_{n}\right) p=p$.

Conversely, if $p$ is finite and $p \leqslant c_{q}$, there is a family $\left\{q_{s}\right\}_{s \in S}$ of mutually orthogonal projections such that $p=\sum_{s \in S} q_{s}$ and $q_{s} \preccurlyeq q$ for all $s \in S$. Let tr: $p \mathcal{M} p \rightarrow \mathcal{Z}(p \mathcal{M} p)=\mathcal{Z}(\mathcal{M}) p$ be the trace on $p \mathcal{M} p$. There are central (in $\mathcal{M}$ ) projections $z_{n, k}^{(s)}$ with $1 \leqslant k \leqslant 2^{n}$ and $n \geqslant 1$ such that

$$
\operatorname{tr}\left(q_{s}\right)=\sum_{n=1}^{\infty}\left(\sum_{k=1}^{2^{n}} \frac{k}{2^{n}} z_{n, k}^{(s)} p\right) .
$$

Since $\operatorname{tr}\left(z_{n, k}^{(s)} p\right) \leqslant 2^{n} \operatorname{tr}\left(q_{s}\right), z_{n, k}^{(s)} p \preccurlyeq 2^{n} \odot q_{s} \preccurlyeq 2^{n} \odot q$. Moreover, we infer from the relation $p=\operatorname{tr}(p)=\sum_{s \in S} \operatorname{tr}\left(q_{s}\right)$ that $\bigvee_{s, n, k} z_{n, k}^{(s)} \geqslant p$. Reindexing the family $\left\{z_{n, k}^{(s)}\right\}_{s, n, k}$ we obtain a collection $\left\{w_{t}\right\}_{t \in T} \subset E(\mathcal{Z}(\mathcal{M}))$ such that

$$
w_{t} p \preccurlyeq m(t) \odot q \quad \text { and } \quad w:=\bigvee_{t \in T} w_{t} \geqslant p
$$

where $m(t)$ is some positive integer. Now let $\left\{v_{t}\right\}_{t \in T}$ be a family of mutually orthogonal central projections such that $v_{t} \leqslant w_{t}(t \in T)$ and $\sum_{t \in T} v_{t}=w$. Let $* \notin T, v_{*}=1-w$ and $m(*)=1$. Observe that $v_{t} p \preccurlyeq m(t) \odot q$ for every $t \in T_{*}:=T \cup\{*\}$, and $\sum_{t \in T_{*}} v_{t}=1$. To this end, define $z_{n}$ for $n>0$ by $z_{n}=\sum\left\{v_{t}: t \in T_{*}, m(t)=n\right\}$.

Let

$$
E_{\omega}(\mathcal{M})=\{q \in E(\mathcal{M}): q \sim \omega \odot p \text { for some finite projection } p\} .
$$

7.4. Lemma. (a) For every $p \in E_{\omega}(\mathcal{M})$ and a properly infinite projection $q \in E(\mathcal{M}), p \preccurlyeq q \Longleftrightarrow p \leqslant c_{q}$.

(b) If $p \in E_{\omega}(\mathcal{M})$ is such that $c_{p}=1$, then $q \sim c_{q} p$ for every $q \in$ $E_{\omega}(\mathcal{M})$

(c) If $p \in E_{\omega}(\mathcal{M})$ and $z \in E(\mathcal{Z}(\mathcal{M}))$, then $z p \in E_{\omega}(\mathcal{M})$.

Proof. Point (c) is immediate and (b) follows from (a) and (c). So, it suffices to check (a). Assume $p$ and $q$ are as there and $p \leqslant c_{q}$. Take a finite projection $p_{0}$ such that $p \sim \omega \odot p_{0}$. By Lemma 7.3, $z_{n} p_{0} \preccurlyeq n \odot q$ for a suitable sequence $\left(z_{n}\right)_{n=1}^{\infty}$ of central projections. Since $q$ is properly infinite, $q \sim \omega \odot q$ and hence $z_{n} p_{0} \preccurlyeq z_{n} q$ which gives $p_{0} \preccurlyeq q$. Consequently, $p \sim \omega \odot p_{0} \preccurlyeq \omega \odot q \sim q$ and we are done.

A steering projection in (a type $\mathrm{II}_{\infty} \mathcal{W}^{*}$-algebra) $\mathcal{M}$ is a projection $p \in E_{\omega}(\mathcal{M})$ with $c_{p}=1$. Since $E_{\omega}(\mathcal{M})$ consists of properly infinite 
projections, Lemma 7.4 ensures that any two steering projections in $\mathcal{M}$ are Murray-von Neumann equivalent.

Now if $\mathcal{M}$ is an arbitrary $\mathcal{W}^{*}$-algebra, the steering projection of $\mathcal{M}$ is defined as the sum of the steering projections of type $\mathrm{I}, \mathrm{II}_{1}, \mathrm{II}_{\infty}$ and III parts of $\mathcal{M}$. It is clear that any two steering projections in $\mathcal{M}$ are Murray-von Neumann equivalent. The reader should also verify that if $p \in E(\mathcal{M})$ is a steering projection, then $c_{p}=1$ and $z p$ is a steering projection of $\mathcal{M} z$ for every central projection $z$ in $\mathcal{M}$.

\section{Decomposition Relative to SteERING PRojection}

Let us first generalize the idea of the previous section. Whenever $\alpha$ is an (arbitrary) cardinal number and $p$ and $q$ are projections in a $\mathcal{W}^{*}$ algebra $\mathcal{M}, p$ is said to be a copy of $q$ provided $p \sim q$; and $p \sim \alpha \odot q$ iff $p$ is a sum of $\alpha$ copies of $q$. In particular, $p \sim 0 \odot q$ is equivalent to $p=0$. When $\mathcal{M}$ contains $\alpha$ mutually orthogonal copies of $q$, we shall also write $p \preccurlyeq \alpha \odot q$ with obvious meaning. Similarly, we shall say that $p$ contains $\alpha$ orthogonal copies of $q$ iff $q^{\prime} \sim \alpha \odot q$ for some projection $q^{\prime} \leqslant p$.

Using standard methods (such as Lemma 6.3.9 and Theorem 6.3.11 of [19]; cf. [1, Proposition III.1.7.1]), similar to those in [13, 14], [36] or [31], one shows the next result (we skip its proof). To simplify its statement, let us define the classes $\Lambda_{I}, \Lambda_{I I}$ and $\Lambda_{I I I}$ as follows. $\Lambda_{I}=$ Card (the class of all cardinals), $\Lambda_{I I}=\operatorname{Card}_{\infty} \cup\{0,1\}$ and $\Lambda_{I I I}=\operatorname{Card}_{\infty} \cup\{0\}$ where $\operatorname{Card}_{\infty}$ is the class of all infinite cardinals. For any cardinal $\alpha$, $\alpha^{+}$is the direct successor of $\alpha$, that is, $\alpha^{+}=\min \{\beta \in$ Card: $\beta>\alpha\}$. Below ' $\sim$ ' refers to the Murray-von Neumann equivalence in $\mathcal{M}$.

8.1. Theorem. Let $\mathcal{M}$ be a properly infinite $\mathcal{W}^{*}$-algebra, $p$ a steering projection of $\mathcal{M}$ and let $\mathcal{A}=p \mathcal{M} p$. Let $z^{I}, z^{I I}, z^{I I I} \in \mathcal{Z}(\mathcal{A})$ be projections such that $z^{I}+z^{I I}+z^{I I I}=p$ and $\mathcal{A} z^{i}$ is of type $i$ for $i=$ $I, I I, I I I$. For every $q \in E(\mathcal{M})$ there is a unique system $\left\{z_{\alpha}^{I}(q)\right\}_{\alpha \in \Lambda_{I}} \cup$ $\left\{z_{\alpha}^{I I}(q)\right\}_{\alpha \in \Lambda_{I I}} \cup\left\{z_{\alpha}^{I I I}(q)\right\}_{\alpha \in \Lambda_{I I I}} \subset \mathcal{Z}(\mathcal{A})$ of mutually orthogonal projections such that for $i=I, I I, I I I, \sum_{\alpha \in \Lambda_{i}} z_{\alpha}^{i}(q)=z^{i}$ and $c_{z_{\alpha}^{i}(q)} q \sim$ $\alpha \odot z_{\alpha}^{i}(q)$ if only $\alpha \in \Lambda_{i}$ and $(i, \alpha) \neq(I I, 1)$, while $c_{z_{1}^{I I}(q)} q$ is finite and $z_{1}^{I I}(q) \sim \omega \odot c_{z_{1}^{I I}(q)} q$.

What is more, $z_{\alpha}^{i}(q)$ may be characterized as follows:

$$
\begin{aligned}
z_{1}^{I I}(q)=\bigvee\left\{w \in E(\mathcal{A}) \mid w \leqslant z^{I I}, \forall v \in E(\mathcal{A}), 0 \neq v \leqslant w:\right. \\
\left.c_{v} q \neq 0 \text { and } q \text { contains no copy of } \omega \odot v\right\}
\end{aligned}
$$

and when $(i, \alpha) \neq(I I, 1)$,

$$
\begin{aligned}
& z_{\alpha}^{i}(q)=\bigvee\left\{w \in E(\mathcal{A}) \mid w \leqslant z^{i}, c_{w} q \sim \alpha \odot w, \forall v \in E(\mathcal{A}):\right. \\
& \left.\quad 0 \neq v \leqslant w \Longrightarrow q \text { does not contain } \alpha^{+} \text {orthogonal copies of } v\right\} .
\end{aligned}
$$


The statement of the above theorem is complicated. We have formulated it in this way for further applications to the class $\mathcal{C D} \mathcal{D}_{N}$.

For purpose of this paper, let us introduce the following

8.2. Definition. Let $i \in\{I, I I, I I I\}$ and $\alpha \in \operatorname{Card}_{\infty}$. A $\mathcal{W}^{*}$-algebra $\mathcal{M}$ is said to be of (pure) type $i_{\alpha}$ iff $\mathcal{M}$ is of pure type $i$ and $1 \sim \alpha \odot p$ where $p$ is the steering projection of $\mathcal{M}$.

Recall that the above definition of type $\mathrm{I}_{\alpha} \mathcal{W}^{*}$-algebras is equivalent to the classical definition of this type, and that below types $\mathrm{I}_{n}$ for finite $n$ and $\mathrm{II}_{1}$ are understood in the usual sense.

8.3. Proposition. For every $\mathcal{W}^{*}$-algebra $\mathcal{M}$ there is a unique system $\left\{z_{\alpha}^{i}: i \in\{I, I I, I I I\}, \alpha \in \Lambda_{i} \backslash\{0\}\right\} \subset E(\mathcal{Z}(\mathcal{M}))$ such that $1=\sum_{i, \alpha} z_{\alpha}^{i}$ and for each $i$ and $\alpha$ either $z_{\alpha}^{i}=0$ or $\mathcal{M} z_{\alpha}^{i}$ is of pure type $i_{\alpha}$.

To simplify statements of next results, we fix $i \in\{I, I I, I I I\}, \gamma \in$ $\operatorname{Card}_{\infty}$, a type $i_{\gamma} \mathcal{W}^{*}$-algebra $\mathcal{M}$ and a steering projection $p$ of $\mathcal{M}$. Additionally, we put $\mathcal{A}=p \mathcal{M} p$ and $\Lambda=\left\{\alpha \in \Lambda_{i}: \alpha \leqslant \gamma\right\}$. For every $q \in E(\mathcal{M})$ let $z_{\alpha}(q)=z_{\alpha}^{i}(q)$ where $z_{\alpha}^{i}(q)$ is as in Theorem 8.1. It is easily seen that $z_{\alpha}(q)=0$ for $\alpha>\gamma$ and $\sum_{\alpha \in \Lambda} z_{\alpha}(q)=p$. Therefore for every $q \in E(\mathcal{M})$ we shall deal with a set $\left\{z_{\alpha}(q)\right\}_{\alpha \in \Lambda}$ of projections.

We skip the proof of the next result (cf. [31]).

8.4. Proposition. For $q, q^{\prime} \in E(\mathcal{M})$ the following conditions are equivalent:

(i) $q \preccurlyeq q^{\prime}$,

(ii) $z_{\beta}(q) z_{\alpha}\left(q^{\prime}\right)=0$ whenever $\alpha, \beta \in \Lambda$ and $\beta>\alpha$; and $c_{z_{1}(q)} c_{z_{1}\left(q^{\prime}\right)} q \preccurlyeq$ $c_{z_{1}(q)} c_{z_{1}\left(q^{\prime}\right)} q^{\prime}$ provided $i=I I$.

The following result explains the terminology proposed by us.

8.5. Proposition. Let $q \in E(\mathcal{M})$ be nonzero. Then $c_{q} \sim \gamma \odot q$ and $\mathcal{M}$ does not contain $\gamma^{+}$orthogonal copies of $q$.

Proof. The second claim is left for the reader. For every positive cardinal $\beta \in \Lambda$ let $S_{\beta}$ be a set such that $\operatorname{card}\left(S_{\beta}\right)=\beta$ and let $\kappa_{\beta}: S_{\gamma} \times S_{\beta} \rightarrow S_{\gamma}$ be a bijection. Since $\mathcal{M}$ is of type $i_{\gamma}$, there is a collection $\left\{p_{s}\right\}_{s \in S_{\gamma}}$ of mutually orthogonal projections Murray-von Neumann equivalent to $p$ which sum up to 1 . For $s \in S_{\gamma}$ let

$$
q_{s}=\sum_{\beta \in \Lambda \backslash\{0\}} c_{z_{\beta}(q)} \sum_{t \in S_{\beta}} p_{\kappa_{\beta}(s, t)} .
$$

Since $c_{z_{\beta}(q)} p_{s} \sim z_{\beta}(q)$ and $\sum_{\beta \in \Lambda \backslash\{0\}} c_{z_{\beta}(q)}=c_{q}, q_{s} \sim q$ for $s \in S_{\gamma}$. To this end, observe that

$$
\sum_{s \in S_{\gamma}} q_{s}=\sum_{\beta \in \Lambda \backslash\{0\}} c_{z_{\beta}(q)} \sum_{(s, t) \in S_{\gamma} \times S_{\beta}} p_{\kappa_{\beta}(s, t)}=\sum_{\beta \in \Lambda \backslash\{0\}} c_{z_{\beta}(q)}=c_{q} .
$$


8.6. Proposition. For every $q \in E(\mathcal{M})$ there are projections $q_{\#}, q^{\#} \in$ $E(\mathcal{M})$ such that $1-q_{\#} \sim q \sim 1-q^{\#}$ and $q_{\#} \preccurlyeq q^{\prime} \preccurlyeq q^{\#}$ for every $q^{\prime} \in E(\mathcal{M})$ with $1-q^{\prime} \sim q$. Moreover, $q^{\#} \sim 1$ and $q_{\#} \sim 1-c_{z_{\gamma}(q)}$.

Proof. Since for $i=I$, III arguments are similar, we shall only sketch the proof for $i=I I$ (which is most complicated). Since $1 \sim 2 \odot 1$, it is clear that there is $q^{\#} \in E(\mathcal{M})$ such that $q^{\#} \sim 1$ and $1-q^{\#} \sim q$. Thus we only need to find $q_{\#}$. For each $\beta \in \Lambda$ let $S_{\beta}$ be a set of cardinality $\beta$ and $\left\{p_{s}\right\}_{s \in S_{\gamma}}$ be a collection of mutually orthogonal projections which are Murray-von Neumann equivalent to $p$ and sum up to 1 . We assume that $S_{\beta} \subset S_{\gamma}$ for each $\beta \in \Lambda$. Let $s_{1} \in S_{1}$. Take $v \in E(\mathcal{M})$ with $v \leqslant p_{s_{1}}$ and $v \sim c_{z_{1}(q)} q$, and put

$$
q_{\#}=c_{z_{1}(q)}\left(p_{s_{1}}-v\right)+\sum_{\beta \in \Lambda} c_{z_{\beta}(q)} \sum_{s \in S_{\gamma} \backslash S_{\beta}} p_{s} .
$$

Since $\sum_{\beta \in \Lambda} c_{z_{\beta}(q)}=1$ and $\operatorname{card}\left(S_{\gamma} \backslash S_{\beta}\right)=\gamma$ if only $\beta<\gamma$, we infer from these that $q_{\#} \sim 1-c_{z_{\gamma}(q)}$. This implies that $z_{\gamma}\left(q_{\#}\right)=\left(1-c_{z_{\gamma}(q)}\right) p=$ $p-z_{\gamma}(q)=\sum_{\beta \in \Lambda \backslash\{\gamma\}} z_{\beta}(q), z_{0}\left(q_{\#}\right)=c_{z_{\gamma}(q)} p=z_{\gamma}(q)$ and $z_{\beta}\left(q_{\#}\right)=0$ for each $\beta \in \Lambda \backslash\{0, \gamma\}$ (in particular, $z_{1}\left(q_{\#}\right)=0$ ). Further, observe that $c_{z_{1}(q)} v=v$ and thus

$$
1-q_{\#}=v+\sum_{\beta \in \Lambda \backslash\{1\}} c_{z_{\beta}(q)} \sum_{s \in S_{\beta}} p_{s}
$$

which yields that $1-q_{\#} \sim q$. Now let $q^{\prime} \in E(\mathcal{M})$ be such that $1-q^{\prime} \sim q$. Thanks to Proposition 8.4, $q_{\#} \preccurlyeq q^{\prime}$ iff $z_{\beta}\left(q_{\#}\right) z_{\alpha}\left(q^{\prime}\right)=0$ whenever $\alpha, \beta \in \Lambda$ and $\alpha<\beta$ (because $z_{1}\left(q_{\#}\right)=0$ ). In our situation the latter is equivalent to $z_{\beta}(q) z_{\alpha}\left(q^{\prime}\right)=0$ for every $\alpha, \beta \in \Lambda \backslash\{\gamma\}$. For such $\alpha$ and $\beta$ we have

$$
w:=c_{z_{\beta}(q)} c_{z_{\alpha}\left(q^{\prime}\right)}=w q^{\prime}+w\left(1-q^{\prime}\right)
$$

and $w\left(1-q^{\prime}\right) \sim w q$. But

$$
\left\{\begin{array} { l l } 
{ w q ^ { \prime } \sim \alpha \odot ( w p ) } & { \text { if } \alpha \neq 1 , } \\
{ w q ^ { \prime } \text { is finite } } & { \text { if } \alpha = 1 , }
\end{array} \text { and } \quad \left\{\begin{array}{ll}
w q \sim \beta \odot(w p) & \text { if } \beta \neq 1 \\
w q \text { is finite } & \text { if } \beta=1 .
\end{array}\right.\right.
$$

We conclude from this that either $w$ is finite (and hence $w=0$ ) or $w \sim \max (\alpha, \beta) \odot w p$. At the same time, thanks to e.g. Proposition 8.5, $w \sim \gamma \odot w p$ which implies that $w=0$ and we are done.

Since in every finite $\mathcal{W}^{*}$-algebra $\mathcal{W}, 1-q^{\prime} \sim q$ iff $q^{\prime} \sim 1-q$ for any $q, q^{\prime} \in E(\mathcal{W})$, Proposition 8.6 gives

8.7. Theorem. Let $\mathcal{W}$ be a $\mathcal{W}^{*}$-algebra and $q \in E(\mathcal{W})$. There are projections $q^{\#}$ and $q_{\#}$ such that $1-q_{\#} \sim q \sim 1-q^{\#}$ and $q_{\#} \preccurlyeq q^{\prime} \preccurlyeq q^{\#}$ whenever $q^{\prime} \in E(\mathcal{W})$ is such that $1-q^{\prime} \sim q$. What is more, if $\mathcal{W}$ is properly infinite, $q^{\#} \sim 1$ and $q_{\#}$ is Murray-von Neumann equivalent to a central projection. 
Our last aim of this section is

8.8. Proposition. Let $S$ be an (infinite) set whose power is a limit cardinal. Let $\left\{p_{s}\right\}_{s \in S}$ be a family of mutually orthogonal projections in a $\mathcal{W}^{*}$-algebra $\mathcal{W}$ which sum up to 1 . For nonempty set $A \subset S$ put $q_{A}=\sum_{s \in A} p_{s}$. Then 1 is the l.u.b. of the family $\left\{q_{A}: A \subset S, 0<\right.$ $\operatorname{card}(A)<\operatorname{card}(S)\}$ with respect to the Murray-von Neumann order.

Proof. Thanks to Proposition 8.3, we may and do assume that $\mathcal{W}$ is of pure type $i_{\gamma}$. Since the assertion of the theorem is known to be true for finite algebras $\mathcal{W}$, we assume in addition that $\mathcal{W}$ is properly infinitethat is, that $\gamma$ is infinite. Finally, we reduce our considerations to the case when the steering projection $p$ of $\mathcal{W}$ is countably decomposable.

Let $q \in E(\mathcal{W})$ be such that $q_{A} \preccurlyeq q$ for each $A \in \mathcal{S}:=\{A \subset S: 0<$ $\operatorname{card}(A)<\operatorname{card}(S)\}$. We need to show that $q \sim 1$. Equivalently, we have to prove that $z_{\alpha}^{i}(q)=0$ provided $\alpha<\gamma$. When $i=I I, c_{z_{1}^{I I}(q)} q$ is finite and $c_{z_{1}^{I I}(q)} \sum_{s \in A} p_{s} \preccurlyeq c_{z_{1}^{I I}(q)} q$ for each $A \in \mathcal{S}$ which implies that $c_{z_{1}^{I I}(q)} \preccurlyeq c_{z_{1}^{I I}(q)} q$. Consequently, $c_{z_{1}^{I I}(q)}$ is finite and thus $z_{1}^{I I}(q)=0$. Also when $i=I$ and $\alpha$ is finite, $z_{\alpha}^{i}(q)=0$, beacuse then $\alpha \odot p$ is finite.

Now we are in position when $\alpha$ is infinite. Then $c_{z_{\alpha}^{i}(q)} q \sim \alpha \odot$ $z_{\alpha}^{i}(q) \sim \alpha \odot\left(c_{z_{\alpha}^{i}(q)} p\right)$ and $c_{z_{\alpha}^{i}(q)} q_{A} \preccurlyeq c_{z_{\alpha}^{i}(q)} q$ for any $A \in \mathcal{S}$. We argue by contradiction. Assume $z_{\alpha}^{i}(q) \neq 0$. Replacing $\mathcal{W}$ by $\mathcal{W} c_{z_{\alpha}^{i}(q)}$, we may assume $c_{z_{\alpha}^{i}(q)}=1$, that is, $z_{\alpha}^{i}(q)=p$. We then have $q \sim \alpha \odot p$, $1 \sim \gamma \odot p$ and $q_{A} \preccurlyeq q(A \in \mathcal{S})$. We distinguish between two cases. When $\operatorname{card}(S) \leqslant \alpha$, we easily get $p_{s} \preccurlyeq q$ and thus $1=\sum_{s \in S} p_{s} \preccurlyeq \alpha \odot q \sim \alpha^{2} \odot p$ which denies the facts that $\alpha^{2}<\gamma$ and $1 \sim \gamma \odot p$.

Finally, assume that card $(S)>\alpha$. Since $p$ is countably decomposable and $q \sim \alpha \odot p, \operatorname{card}\left(\left\{s \in A: p_{s} \neq 0\right\}\right) \leqslant \alpha$ for any $A \in \mathcal{S}$ (because $\left.q_{A} \preccurlyeq q\right)$. We conclude from this that $A:=\left\{s \in S: p_{s} \neq 0\right\} \in \mathcal{S}$ (because $\operatorname{card}(S)>\alpha^{+}$). But then $1=q_{A} \preccurlyeq q$ and we are done.

8.9. Example. As the following example shows (compare with [36, Example 3]), the assumption in Proposition 8.8 that the power of $S$ is a limit cardinal is essential. Let $\mathcal{H}$ be a Hilbert space of dimension $\aleph_{1}, S$ be a set of power $\aleph_{1}$ and let $\left\{e_{s}\right\}_{s \in S}$ be an orthonormal basis of $\mathcal{H}$. Further, let $\mathcal{M}=\mathcal{B}(\mathcal{H})$ and for $s \in S$ let $p_{s} \in E(\mathcal{M})$ be the orthogonal rank-one projection onto the linear span of $e_{s}$. Now if $q_{A}$ 's are defined as in Proposition 8.8, then $q_{A} \preccurlyeq q_{J}$ for every nonempty set $A \subset S$ of power less than $\aleph_{1}$ where $J$ is a countable infinite subset of $S$ and hence 1 is nonequivalent to the l.u.b. (which is $q_{J}$ ).

\section{Minimal AND SEMiminimal $N$-TUPles}

The idea of steering projections will now be adapted to the class $\mathcal{C D D}_{N}$. Following Ernest [9], we say a nontrivial $N$-tuple $\mathbb{A} \in \mathcal{C D} \mathcal{D}_{N}$ is (of) type I, II, III iff such is $\mathcal{W}^{\prime}(\boldsymbol{A})$. Additionally, we let the trivial $N$-tuple be of each of these types. 
We begin with a result which will find many applications in the sequel.

9.1. Lemma. Every collection of mutually unitarily disjoint nontrivial members of $\mathcal{C D D}_{N}$ has power no greater than $2^{\aleph_{0}}$.

Proof. Suppose

$$
\mathbb{A}^{(s)} \perp_{u} \mathbb{A}^{\left(s^{\prime}\right)}
$$

(and $\left.\mathbb{A}^{(s)} \neq \mathbb{O}\right)$ for distinct $s, s^{\prime} \in S$. For $n \in J=\{1,2,3, \ldots\} \cup\left\{\aleph_{0}\right\}$ let $\mathcal{H}_{n}$ be a fixed Hilbert space of dimension $n$. By Theorem 3.4 (page 8), for each $s \in S$ there is $n(s) \in J$ and $\boldsymbol{B}^{(s)} \in \mathrm{CDD}_{N}\left(\mathcal{H}_{n(s)}\right)$ such that $\mathbb{B}^{(s)} \leqslant \mathbb{A}^{(s)}$. We infer from $(9-1)$ that $\boldsymbol{B}^{(s)} \neq \boldsymbol{B}^{\left(s^{\prime}\right)}$ for distinct $s, s^{\prime} \in S$. Now the assertion easily follows from the fact that card $\left(\operatorname{CDD}_{N}\left(\mathcal{H}_{n}\right)\right) \leqslant$ $2^{\aleph_{0}}$ for every $n \in J$.

9.2. Definition. $\mathbb{A} \in \mathcal{C D} \mathcal{D}_{N}$ is said to be minimal iff for every $\mathbb{B} \in$ $\operatorname{eDD}_{N}$,

$$
\mathbb{A} \ll \mathbb{B} \Longrightarrow \mathbb{A} \leqslant \mathbb{B} .
$$

$\mathbb{A}$ is said to be multiplicity free $\left(\mathbb{A} \in \mathcal{M} \mathcal{F}_{N}\right)$ iff there is no nontrivial $\mathbb{B} \in \mathcal{C D D}_{N}$ for which $2 \odot \mathbb{B} \leqslant \mathbb{A}$. $\mathbb{A}$ is a hereditary idempotent $(\mathbb{A} \in$ $\left.\mathcal{H J}_{N}\right)$ iff $\mathbb{B}=2 \odot \mathbb{B}$ for every $\mathbb{B} \leqslant \mathbb{A}$. We shall write $\mathbb{A} \in \mathcal{H}_{\mathcal{T}} \mathcal{M}_{N}$ to express that $\mathbb{A}$ is both a hereditary idempotent and minimal.

Minimal members of $\mathcal{C D} \mathcal{D}_{N}$ correspond to quasi-abelian projections.

9.3. Remark. The work of Ernest [9] deals with (single) bounded operators. In this context, our definition of a multiplicity free operator is equivalent to Ernest's one (Definition 1.21 in [9]).

9.4. Theorem. (I) For every $\mathbb{A} \in \mathcal{C D} \mathcal{D}_{N}$,

$$
\mathbb{A}=2 \odot \mathbb{A} \Longleftrightarrow \mathbb{A}=\aleph_{0} \odot \mathbb{A} \text {. }
$$

(II) For $\mathbb{A} \in \mathcal{C D D}_{N}$ the following conditions are equivalent:

(i) $\mathbb{A}$ is minimal,

(ii) for each $\mathbb{B} \in \mathcal{C D} \mathcal{D}_{N}, \mathbb{B} \leqslant \mathbb{A} \Longrightarrow \mathbb{B} \leqslant{ }^{s} \mathbb{A}$.

If $\mathbb{A}$ is minimal and $\mathbb{B} \leqslant \mathbb{A}$, then $\mathbb{B}$ is minimal as well.

(III) For $\mathbb{A} \in \mathcal{C D D}_{N}$ the following conditions are equivalent:

(i) $\mathbb{A} \in \mathcal{M} \mathcal{F}_{N}$,

(ii) $\mathbb{A}=\mathbb{O}$ or $\mathcal{W}^{\prime}(\boldsymbol{A})$ is commutative.

In particular, if $\mathbb{A} \in \mathcal{M} \mathcal{F}_{N}$ and $\mathbb{B} \leqslant \mathbb{A}$, then $\mathbb{B} \in \mathcal{M} \mathcal{F}_{N}$ as well.

(IV) Every multiplicity free $N$-tuple is minimal and unitarily disjoint from any hereditary idempotent.

(V) If $\mathbb{A} \in \mathcal{H}_{N}$ and $\mathbb{B} \ll \mathbb{A}$, then $\mathbb{B} \in \mathcal{H}_{N}$ as well.

(VI) There exist unique $\mathbb{J}_{I}, \mathbb{J}_{I I I} \in \mathcal{C D} \mathcal{D}_{N}$ such that $\mathbb{J}_{I} \in \mathcal{M} \mathcal{F}_{N}, \mathbb{J}_{I I I} \in$ $\mathcal{H} \mathcal{J M}_{N}, \mathbb{J}_{I} \boxplus \mathbb{J}_{I I I}$ is minimal and for every $\mathbb{A} \in \mathcal{C D} \mathcal{D}_{N}$ :

(a) $\mathbb{A} \in \mathcal{M} \mathcal{F}_{N}$ iff $\mathbb{A} \leqslant \mathbb{J}_{I}$,

(b) $\mathbb{A} \ll \mathbb{J}_{I}$ iff $\mathbb{A}=\mathbb{O}$ or $\mathcal{W}^{\prime}(\boldsymbol{A})$ is type $I$, 
(c) $\mathbb{A} \in \mathcal{H J}_{N}$ iff $\mathbb{A} \ll \mathbb{J}_{I I I}$, iff $\mathbb{A}=\mathbb{O}$ or $\mathcal{W}^{\prime}(A)$ is type III,

(d) $\mathbb{A} \in \mathcal{H} \mathcal{J M M}_{N}$ iff $\mathbb{A} \leqslant \mathbb{J}_{I I I}$,

(e) $\mathbb{A}$ is minimal iff $\mathbb{A} \leqslant \mathbb{J}_{I} \boxplus \mathbb{J}_{I I I}$.

What is more, $\operatorname{dim}\left(\mathbb{J}_{I}\right)+\operatorname{dim}\left(\mathbb{J}_{I I I}\right) \leqslant 2^{\aleph_{0}}$.

Proof. In all points of the theorem we make use of Proposition 4.1 (page 9). Counterparts of points (I) and (V) are well known for projections in $\mathcal{W}^{*}$-algebras, (II) follows from Lemma 7.1 (page 15), (III) is immediate, (IV) is implied by (III) and the definitions of suitable notions. We shall describe how to prove (VI). Take a maximal collection (cf. Lemma 9.1) of nontrivial mutually unitarily disjoint multiplicity free $N$-tuples (respectively hereditary idempotents) whose representatives act in separable spaces and define $\mathbb{J}_{I}\left(\mathbb{J}_{I I I}\right)$ as the direct sum of this family. One may check that obtained in this way $N$-tuple belongs to $\mathcal{N} \mathcal{F}_{N}\left(\mathcal{H J M}_{N}\right)$ and - since $\mathbb{J}_{I}$ and $\mathbb{J}_{I I I}$ are unitarily disjoint - that $\mathbb{J}_{I} \boxplus \mathbb{J}_{I I I}$ is minimal. That $\mathbb{J}_{I}$ and $\mathbb{J}_{I I I}$ are greatest members of $\mathcal{M} \mathcal{F}_{N}$ and $\mathcal{H J M M}_{N}$ it follows from the maximality of the taken families and Theorem 3.4 (page 8). The details are left for the reader (cf. Propositions 2.12, 1.27 and 1.29 and Corollary 1.37 in [9]). (For the proof of (b) and (c) see also Theorem 11.1, page 26.)

Theorem 9.4 shows that there is a greatest minimal $N$-tuple in CDDD $\mathcal{D}_{N}$, namely $\mathbb{J}_{I} \boxplus \mathbb{J}_{I I I}$, and that it covers all type I and III $N$-tuples. Since there are also type II ones, we need to introduce one more notion.

9.5. Definition. $\mathbb{A} \in \mathcal{C D} \mathcal{D}_{N}$ is said to be semiminimal $\left(\mathbb{A} \in \mathcal{S M}_{N}\right)$ iff $\mathbb{A}$ is unitarily disjoint from every minimal $N$-tuple and $\mathbb{A}$ satisfies the following condition. Whenever $\mathbb{B} \in \mathcal{C D} \mathcal{D}_{N}$ is such that $\mathbb{A} \ll \mathbb{B}, \mathbb{A}$ may be written in the form $\mathbb{A}=\bigoplus_{n=1}^{\infty} \mathbb{A}_{n}$ where $\mathbb{A}_{n} \leqslant n \odot \mathbb{B}$ for each $n \geqslant 1$.

Before stating the next result, we underline that there is no greatest semiminimal member of $\mathcal{C D} \mathcal{D}_{N}$.

9.6. Theorem. (I) For $\mathbb{A} \in \mathcal{C D} \mathcal{D}_{N}, \mathbb{A} \in \mathcal{S} \mathcal{M}_{N}$ iff $\mathbb{A}=\mathbb{O}$ or $\mathcal{W}^{\prime}(\boldsymbol{A})$ is type $I I_{1}$. In particular, if $\mathbb{A} \in \mathcal{S M}_{N}$ and $\mathbb{B} \leqslant \mathbb{A}$, then $\mathbb{B} \in \mathcal{S \mathcal { M }}_{N}$ as well; the direct sum of finitely many semiminimal $N$-tuples belongs to $\mathcal{S M}_{N}$.

(II) There is unique $\mathbb{J}_{I I} \in \mathcal{C D} \mathcal{D}_{N}$ such that for every $\mathbb{A} \in \mathcal{S M}_{N}$ there is $\mathbb{B} \in \mathcal{S \mathcal { M }}_{N}$ for which $\mathbb{J}_{I I}=\aleph_{0} \odot(\mathbb{A} \boxplus \mathbb{B})$. Moreover, $\operatorname{dim}\left(\mathbb{J}_{I I}\right) \leqslant 2^{\aleph_{0}}$ and

(a) for $\mathbb{E}, \mathbb{F} \in \mathcal{C D D}_{N}$ with $\mathbb{E} \leqslant \mathbb{F} \leqslant \mathbb{I}_{I I}$,

$\mathbb{E} \leqslant \leqslant^{s} \mathbb{F} \leqslant^{s} \mathbb{J}_{I I} \Longleftrightarrow \mathbb{E}=2 \odot \mathbb{E}$ and $\mathbb{F}=2 \odot \mathbb{F}$,

(b) $\mathbb{A} \ll \mathbb{J}_{I I}$ iff $\mathbb{A}=\mathbb{O}$ or $\mathcal{W}^{\prime}(\boldsymbol{A})$ is type $I I$.

Proof. Point (I) follows from Lemma 7.3 (page 16) and Theorem 9.4 from which we infer that $\mathcal{W}^{\prime}(A)$ is type II for every $\mathbb{A} \in \mathcal{S} \mathcal{M}_{N}$ (because every semiminimal $N$-tuple is unitarily disjoint from $\left.\mathbb{J}_{I} \boxplus \mathbb{J}_{I I I}\right)$. To prove (II), proceed similarly as in the proof of Theorem 9.4. Take a 
maximal family $\mathcal{A}$ of mutually unitarily disjoint nontrivial members of $\mathcal{S M}_{N}$ whose representatives act in separable spaces and denote by $\mathbb{S}(\mathcal{A})$ its direct sum. Next put $\mathbb{J}_{I I}=\aleph_{0} \odot \mathbb{S}(\mathcal{A})$. Check that $\mathbb{S}(\mathcal{A}) \in \mathcal{S M}_{N}$ for every such $\mathcal{A}$. Further, show that for two maximal families $\mathcal{A}$ and $\mathcal{A}^{\prime}$ one has $\mathbb{S}(\mathcal{A}) \ll \mathbb{S}\left(\mathcal{A}^{\prime}\right) \ll \mathbb{S}(\mathcal{A})$ and consequently, by the definition of semiminimality, $\aleph_{0} \odot \mathbb{S}\left(\mathcal{A}^{\prime}\right)=\aleph_{0} \odot \mathbb{S}(\mathcal{A})$. Having this, one easily shows the uniqueness of $\mathbb{J}_{I I}$ and all suitable properties of it. (For example, if $\mathbb{E}=2 \odot \mathbb{E}$, then $\mathbb{E}=\aleph_{0} \odot \mathbb{E}$ and it suffices to apply Lemma 7.4, page 17.)

The reader should notice that $\mathbb{J}_{I I}$ corresponds to the steering projection of a type $\mathrm{II}_{\infty} \mathcal{W}^{*}$-algebra.

9.7. Remark. Point (II) of Theorem 9.6 implies that $\mathbb{J}_{I I}$ is the greatest element of the class $\mathcal{S} \mathcal{M}_{N}^{\infty}=\left\{\aleph_{0} \odot \mathbb{A}: \mathbb{A} \in \mathcal{S} \mathcal{M}_{N}\right\}$ (and hence $\mathcal{S} \mathcal{M}_{N}^{\infty}$ is a set) and that for any $\mathbb{A}, \mathbb{B} \in \mathcal{S \mathcal { M }}_{N}^{\infty}, \mathbb{A} \leqslant \mathbb{B} \Longleftrightarrow \mathbb{A} \leqslant s$.

Let us denote by $\mathbb{J}$ the $N$-tuple $\mathbb{J}_{I} \boxplus \mathbb{J}_{I I} \boxplus \mathbb{J}_{I I I}$. We call $\mathbb{I}$ the unity of $\mathcal{C D D}_{N}$. Since every $\mathcal{W}^{*}$-algebra admits a decomposition into type I, II and III parts, this yields

9.8. Proposition. For every $\mathbb{A} \in \mathcal{C D} \mathcal{D}_{N}, \mathbb{A} \ll \mathbb{J}$.

9.9. Remark. It may be worthwhile to note that $\operatorname{dim}\left(\mathbb{J}_{i}\right)=2^{\aleph_{0}}$ for $i=I, I I, I I I$. We shall prove this later (see Corollary 17.9, page 62). We conclude from this and Proposition 9.8 that for an infinite cardinal $\alpha$ there exists $\mathbb{A} \in \mathcal{C D} \mathcal{D}_{N}$ such that $\operatorname{dim}(\mathbb{A})=\alpha$ and $\mathbb{X} \leqslant \mathbb{A}$ whenever $\operatorname{dim}(\mathbb{X}) \leqslant \alpha$ iff $\alpha \geqslant 2^{\aleph_{0}}$. If the latter happens, such $\mathbb{A}$ is of course unique and one may check that $\mathbb{A}=\alpha \odot \mathbb{J}$.

In the sequel we shall also need the following

9.10. Proposition. For every nontrivial $\mathbb{A} \leqslant \mathbb{J}$ there is $\mathbb{B} \leqslant s \mathbb{A}$ such that $0<\operatorname{dim}(\mathbb{B}) \leqslant \aleph_{0}$.

Proof. By Theorem 3.4 (page 8), there is nontrivial $\mathbb{B}_{0} \leqslant \mathbb{A}$ such that $\boldsymbol{B}_{0}$ acts in a separable Hilbert space. We may assume that $\mathbb{B}_{0} \leqslant \mathbb{J}_{i}$ for some $i \in\{I, I I, I I I\}$. If $i \neq I I$, we automatically have $\mathbb{B}_{0} \leqslant s \mathbb{A}$; while when $i=I I$, it suffices to notice that $\aleph_{0} \odot \mathbb{B}_{0} \leqslant^{s} \aleph_{0} \odot \mathbb{A}$ (by (9-2)) and to apply (PR6) (page 9) to find $\mathbb{B} \leqslant{ }^{s} \mathbb{A}$ with $\aleph_{0} \odot \mathbb{B}=\aleph_{0} \odot \mathbb{B}_{0}$.

9.11. Example. When $N=1$, one may check that a bounded normal operator on a separable Hilbert space is multiplicity free iff it is *cyclic (an operator $T \in \mathcal{B}(\mathcal{H})$ is $*$-cyclic iff there is $x \in \mathcal{H}$ for which the linear span of $\{x\} \cup\left\{S_{1} \ldots S_{m} x: m \geqslant 1, S_{1}, \ldots, S_{m} \in\left\{T, T^{*}\right\}\right\}$ is dense in $\mathcal{H}$ ). Taking this into account, one may ask whether every *-cyclic type I operator is multiplicity free. As this simple example shows, it is untrue. Let $T=\left(\begin{array}{ll}0 & 0 \\ 1 & 0\end{array}\right)$ and $S=T \oplus T$. Of course, $S \notin \mathcal{M} \mathcal{F}_{1}$. However, $S$ is $*$-cyclic. (For $u=(1,0,0,1), S u=(0,1,0,0)$, $S^{*} u=(0,0,1,0)$ and $S^{*} S u=(1,0,0,0)$.) 


\section{UNITIES OF IDEALS IN $\mathcal{C D} \mathcal{D}_{N}$}

Reformulating conditions (ID1)-(ID4) (page 10) into the terms of $\mathcal{C D} \mathcal{D}_{N}$, we obtain the notion of an ideal in $\mathcal{C D} \mathcal{D}_{N}$. Equivalently, a nonempty class $\mathcal{A} \subset \mathcal{C D D} \mathcal{D}_{N}$ is an ideal provided $\mathcal{A}$ is order-complete (i.e. $\bigvee \mathcal{F} \in \mathcal{A}$ for every nonempty set $\mathcal{F} \subset \mathcal{A}$ ) and $\mathfrak{m} \odot \mathbb{A} \in \mathcal{A}$ whenever $\mathfrak{m}$ is a cardinal and $\mathbb{A} \leqslant \mathbb{B}$ for some $\mathbb{B} \in \mathcal{A}$.

Theorem 5.1 (page 10) asserts that for every ideal $\mathcal{A} \subset \mathcal{C D} \mathcal{D}_{N}$ and $\mathbb{X} \in \mathcal{C D D}_{N}$ there is unique $\mathbb{Y} \in \mathcal{A}$ such that $\mathbb{Y} \leqslant \leqslant^{s} \mathbb{X}$ and $\mathbb{X} \boxminus \mathbb{Y} \in \mathcal{A}^{\perp}$. We shall denote this unique $\mathbb{Y}$ by $\mathbb{E}(\mathbb{X} \mid \mathcal{A})$. Similarly, if $\mathbb{A}$ is any member of $\mathcal{C D D} \mathcal{D}_{N}, \mathbb{E}(\mathbb{X} \mid \mathbb{A}):=\mathbb{E}(\mathbb{X} \mid\{\mathbb{B}: \mathbb{B} \ll \mathbb{A}\})$. $\mathbb{E}(\mathbb{X} \mid \mathbb{A})$ is Ernest's $\mathbb{A}$-shadow of $\mathbb{X}$ (see $[9$, Definition 2.13]).

One may easily verify that

$$
\mathbb{E}\left(\bigoplus_{s \in S} \mathbb{X}^{(s)} \mid \mathcal{A}\right)=\bigoplus_{s \in S} \mathbb{E}\left(\mathbb{X}^{(s)} \mid \mathcal{A}\right)
$$

for every ideal $\mathcal{A} \subset \mathcal{C D} \mathcal{D}_{N}$ and any family $\left\{\mathbb{X}^{(s)}\right\}_{s \in S} \subset \mathcal{C D} \mathcal{D}_{N}$. We shall use the above property several times in the sequel.

Let $\mathcal{A}$ be an ideal in $\mathcal{C D D}_{N}$. The $N$-tuple $\mathbb{J}(\mathcal{A}):=\mathbb{E}(\mathbb{J} \mid \mathcal{A})$ is uniquely determined by $\mathcal{A}$ and is called the unity of $\mathcal{A}$. Proposition 9.8 implies that

10.1. Proposition. For every ideal $\mathcal{A}$ in $\operatorname{CDD}_{N}$,

$$
\mathcal{A}=\left\{\mathbb{X} \in \mathcal{C D D D}_{N}: \mathbb{X} \ll \mathbb{J}(\mathcal{A})\right\},
$$

$\mathbb{J}(\mathcal{A}) \leqslant^{s} \mathbb{J}$ and $\mathbb{J}(\mathcal{A})=\bigvee\{\mathbb{A} \leqslant \mathbb{I}: \mathbb{A} \in \mathcal{A}\}$.

10.2. Corollary. There is a one-to-one correspondence between ideals in $\mathcal{C D D}_{N}$ and members $\mathbb{A}$ of $\mathcal{C D D}_{N}$ such that $\mathbb{A} \leqslant s \mathbb{J}$. The correspondence is established by assignments $\mathcal{A} \mapsto \mathbb{J}(\mathcal{A})$ and $\mathbb{A} \mapsto\{\mathbb{B}: \mathbb{B} \ll \mathbb{A}\}$. In particular, there is at most $2^{2^{\aleph_{0}}}$ ideals in $\mathcal{C D D}_{N}$.

10.3. Example. Let $\mathcal{N}_{N} \subset \mathcal{C D} \mathcal{D}_{N}$ be the ideal of all normal $N$-tuples (see Examples 5.3-(E), page 12). Since $\mathcal{W}^{\prime \prime}(\boldsymbol{M})$ is commutative for every $\mathbb{M} \in \mathcal{N}_{N}, \mathcal{W}^{\prime}(\boldsymbol{M})$ is type I and hence $\mathbb{M} \ll \mathbb{J}_{I}$. Here we shall give a description for $\mathbb{J}\left(\mathcal{N}_{N}\right)$. First of all, $\mathbb{M} \leqslant \mathbb{I}$ iff $\mathcal{W}^{\prime}(\boldsymbol{M})$ is commutative (provided $\mathbb{M} \neq \mathbb{O}$ and $\mathbb{M} \in \mathcal{N}_{N}$ ). When $M$ acts in a separable Hilbert space, the latter is equivalent to the fact that $\boldsymbol{M}$ is $*$-cyclic. That is, there has to exist $x \in \overline{\mathcal{D}}(\boldsymbol{M})$ such that the smallest reducing subspace for $\boldsymbol{M}$ which contains $x$ coincides with $\overline{\mathcal{D}}(\boldsymbol{M})$. (Indeed, if $\mathbb{M} \in \mathcal{M} \mathcal{F}_{N} \cap$ $\mathcal{N}_{N}$ is such that $0<\operatorname{dim}(\mathbb{M}) \leqslant \aleph_{0}$, then both $\mathcal{W}^{\prime}(\boldsymbol{M})$ and $\mathcal{W}^{\prime \prime}(\boldsymbol{M})$ are commutative which means that $\mathcal{W}^{\prime \prime}(\boldsymbol{M})$ is a MASA and consequenty $\mathcal{W}^{\prime \prime}(\boldsymbol{M})$ is cyclic or, equivalently, $\boldsymbol{M}$ is $*$-cyclic. Conversely, if $\mathbb{M} \in$ $\mathcal{N}_{N}$ and $\boldsymbol{M}$ is $*$-cyclic, then $\boldsymbol{M}$ is unitarily equivalent to $\boldsymbol{M}_{\mu}$ for some probabilistic Borel measure $\mu$ on $\mathbb{C}^{N}$ where $\boldsymbol{M}_{\mu}=\left(M_{z_{1}}, \ldots, M_{z_{N}}\right)$ and $M_{z_{j}}$ is the multiplication operator by $z_{j}$ in $L^{2}(\mu)$. One may show that $\mathcal{W}^{\prime}\left(\boldsymbol{M}_{\mu}\right)$ coincides with the algebra of all multiplication operators by 
members of $L^{\infty}(\mu)$ and hence $\mathbb{M} \in \mathcal{M} \mathcal{F}_{N}$.) Having this, one shows that $\mathbb{J}\left(\mathcal{N}_{N}\right)$ may be represented as follows. Take a maximal family $\left\{\mu_{s}\right\}_{s \in S}$ of mutually orthogonal probabilistic Borel measures on $\mathbb{C}^{N}$. For each $s \in S$ let $\boldsymbol{M}^{(s)}=\boldsymbol{M}_{\mu_{s}}$ (defined as before). One may check that $\mathbb{J}\left(\mathcal{N}_{N}\right)=$ $\boxplus_{s \in S} \mathbb{M}^{(s)}$. Moreover, for two probabilistic Borel measures $\mu$ and $\lambda$ on $\mathbb{C}^{N}$ : (a) $M_{\mu} \leqslant M_{\lambda} \Longleftrightarrow \mu \ll \lambda$; (b) $\boldsymbol{M}_{\mu} \equiv \boldsymbol{M}_{\lambda} \Longleftrightarrow \mu \ll \lambda \ll \mu$; (c) $\boldsymbol{M}_{\mu} \perp_{u} \boldsymbol{M}_{\lambda} \Longleftrightarrow \mu \perp \lambda$. Similar (and more detailed) construction will appear in Section 22 .

Theorems 9.4 and 9.6 show that for $i \in\{I, I I, I I I\}$ the ideal $\mathcal{J}_{i}=$ $\left\{\mathbb{X} \in \mathcal{C D D}_{N}: \mathbb{X} \ll \mathbb{J}_{i}\right\}$ consists of all $N$-tuples of type $i$.

\section{Decomposition Relative to $\mathbb{J}$}

Recall that $\Lambda_{I}=$ Card, $\Lambda_{I I}=\operatorname{Card}_{\infty} \cup\{0,1\}$ and $\Lambda_{I I I}=\operatorname{Card}_{\infty} \cup\{0\}$. For simplicity, let $\Upsilon=\left\{(i, \alpha): i \in\{I, I I, I I I\}, \alpha \in \Lambda_{i}\right\}$ and $\Upsilon_{*}=$ $\Upsilon \backslash\{(I I, 1)\}$.

11.1. Theorem. For every $\mathbb{A} \in \mathcal{C D} \mathcal{D}_{N}$ there are a unique regular collection $\left\{\mathbb{E}_{\alpha}^{i}(\mathbb{A}):(i, \alpha) \in \Upsilon\right\}$ and unique $\mathbb{E}_{\text {sm }}(\mathbb{A}) \in \mathcal{C D D}_{N}$ such that for $i \in\{I, I I, I I I\}$,

$$
\mathbb{J}_{i}=\bigoplus_{\alpha \in \Lambda_{i}} \mathbb{E}_{\alpha}^{i}(\mathbb{A})
$$

$\mathbb{E}_{\text {sm }}(\mathbb{A})$ is semiminimal and $\mathbb{E}_{1}^{I I}(\mathbb{A})=\aleph_{0} \odot \mathbb{E}_{\text {sm }}(\mathbb{A})$, and

$$
\mathbb{A}=\mathbb{E}_{s m}(\mathbb{A}) \boxplus \bigoplus_{(i, \alpha) \in \Upsilon_{*}} \alpha \odot \mathbb{E}_{\alpha}^{i}(\mathbb{A}) .
$$

What is more, $\mathbb{E}_{\text {sm }}(\mathbb{A})=\mathbb{A} \wedge \mathbb{E}_{1}^{I I}(\mathbb{A})$ and $\mathbb{E}_{\alpha}^{i}(\mathbb{A})$ 's may be characterized as follows:

$$
\mathbb{E}_{1}^{I I}(\mathbb{A})=\bigvee\left\{\mathbb{E} \leqslant \mathbb{J}_{I I} \mid \mathbb{E} \ll \mathbb{A}, \forall \mathbb{F} \leqslant \mathbb{E}, \mathbb{F} \neq \mathbb{O}: \aleph_{0} \odot \mathbb{F} \nless \mathbb{A}\right\}
$$

and for $(i, \alpha) \in \Upsilon_{*}$,

$$
\mathbb{E}_{\alpha}^{i}(\mathbb{A})=\bigvee\left\{\mathbb{E} \leqslant \mathbb{J}_{i} \mid \alpha \odot \mathbb{E} \leqslant \mathbb{A}, \forall \mathbb{F} \leqslant \mathbb{E}, \mathbb{F} \neq \mathbb{O}: \alpha^{+} \odot \mathbb{F} \nless \mathbb{A}\right\} .
$$

Proof. Thanks to Proposition 9.8, there is an infinite cardinal $\gamma$ such that $\mathbb{A} \leqslant \gamma \odot \mathbb{J}=: \mathbb{B}$. Put $\mathcal{M}=\mathcal{W}^{\prime}(\boldsymbol{B})$, observe that $\boldsymbol{J}$ corresponds (by Proposition 4.1, page 9) to a steering projection of $\mathcal{M}$ and apply Theorem 8.1 (page 18). (Use Theorem 9.6 to deduce that suitable $\mathbb{E}_{s m}(\mathbb{A})$ is semiminimal. Note that if $\mathbb{X}$ and $\mathbb{Y}$ correspond, by Proposition 4.1, to projections $p$ and $q$, then $p \sim \alpha \odot q$ is equivalent to $\mathbb{X}=\alpha \odot \mathbb{Y}$.)

The system $\left\{\mathbb{E}_{\alpha}^{i}(\mathbb{A}):(i, \alpha) \in \Upsilon\right\}$ appearing in Theorem 11.1 is said to be the partition of unity induced by $\mathbb{A}$. (In general, a partition of unity is any regular collection $\left\{\mathbb{E}^{(j)}\right\}_{j \in I}$ such that $\mathbb{J}=\mathbb{\boxplus}_{j \in I} \mathbb{E}^{(j)}$. Note that in that case $\mathbb{E}^{(j)} \leqslant^{s} \mathbb{J}$ for each $j \in I$.) 
11.2. Remark. Theorem 11.1 may be formulated in an equivalent manner that after fixing a representative $\boldsymbol{J}$ for $\mathbb{J}$ for every $\boldsymbol{A} \in \mathrm{CDD}_{N}$ there are unique systems $\left\{H_{\alpha}^{i}:(i, \alpha) \in \Upsilon\right\} \subset \operatorname{cred}(\boldsymbol{J})$ and $\left\{K_{\alpha}^{i}:(i, \alpha) \in\right.$ $\Upsilon\} \subset \operatorname{cred}(\boldsymbol{A})$ such that $\overline{\mathcal{D}}\left(\boldsymbol{J}_{i}\right)=\bigoplus_{\alpha \in \Lambda_{i}} H_{\alpha}^{i}$ for $i \in\{I, I I, I I I\} ; \overline{\mathcal{D}}(\boldsymbol{A})=$ $\bigoplus_{(i, \alpha) \in \Upsilon} K_{\alpha}^{i} ; \mathcal{W}^{\prime}\left(\left.A\right|_{K_{1}^{I I}}\right)$ is type $\mathrm{II}_{1},\left.\left.\aleph_{0} \odot A\right|_{K_{1}^{I I}} \equiv \boldsymbol{J}\right|_{H_{1}^{I I}}$ and for every $(i, \alpha) \in \Upsilon_{*}$,

$$
\left.\left.\boldsymbol{A}\right|_{K_{\alpha}^{i}} \equiv \alpha \odot \boldsymbol{J}\right|_{H_{\alpha}^{i}}
$$

(In particular, $K_{0}^{I}, K_{0}^{I I}$ and $K_{0}^{I I I}$ are trivial.)

As an immediate consequence of Proposition 8.4 (page 19) we obtain

11.3. Proposition. For any $\mathbb{A}, \mathbb{B} \in \mathcal{C D} \mathcal{D}_{N}, \mathbb{A} \leqslant \mathbb{B}$ iff $\mathbb{E}_{\alpha}^{i}(\mathbb{A}) \perp_{u} \mathbb{E}_{\beta}^{i}(\mathbb{B})$ whenever $(i, \alpha),(i, \beta) \in \Upsilon$ and $\alpha>\beta$; and $\mathbb{E}_{\text {sm }}(\mathbb{A}) \wedge \mathbb{E}_{1}^{I I}(\mathbb{B}) \leqslant \mathbb{E}_{\text {sm }}(\mathbb{B})$.

One may also show that

11.4. Proposition. For any $\mathbb{A}, \mathbb{B} \in \mathcal{C D} \mathcal{D}_{N}, \mathbb{A} \leqslant s$ iff $\mathbb{E}_{\alpha}^{i}(\mathbb{A}) \leqslant \mathbb{E}_{\alpha}^{i}(\mathbb{B})$ whenever $(i, \alpha) \in \Upsilon$ is such that $\alpha \neq 0$, and $\mathbb{E}_{s m}(\mathbb{A}) \leqslant \mathbb{E}_{s m}(\mathbb{B})$.

The proofs of Propositions 11.3 and 11.4 are skipped.

Another interesting consequences of Theorem 11.1 are stated below.

11.5. Corollary. Let $\mathbb{A}, \mathbb{B} \in \mathcal{C D} \mathcal{D}_{N}$ and let $\alpha$ be an arbitrary infinite cardinal number such that $\alpha \geqslant \max (\operatorname{dim}(\mathbb{A}), \operatorname{dim}(\mathbb{B}))$.

(I) $\mathbb{A} \ll \mathbb{B} \Longleftrightarrow \alpha \odot \mathbb{A} \leqslant^{s} \alpha \odot \mathbb{B}$.

(II) $\mathbb{A} \ll \mathbb{B} \ll \mathbb{A} \Longleftrightarrow \alpha \odot \mathbb{A}=\alpha \odot \mathbb{B}$.

Proof. In both the points implication ' $\Longleftarrow$ ' is immediate. Conversely, observe that for each $\mathbb{X} \in \mathcal{C D D} \mathcal{D}_{N}$ and $(i, \beta) \in \Upsilon, \mathbb{E}_{\beta}^{i}(\mathbb{X})=\mathbb{O}$ provided $\beta>\operatorname{dim}(\mathbb{X})$. This implies that if $\beta \geqslant \max \left(\aleph_{0}, \operatorname{dim}(\mathbb{X})\right)$, then $\beta \odot \mathbb{X}=$ $\beta \odot \mathbb{E}$ for some $\mathbb{E} \leqslant^{s} \mathbb{J}$. This notice yields the inverse implication in both points (I) and (II). (Observe that if $\mathbb{E}^{\prime} \leqslant s \mathbb{E}^{\prime \prime}$, then $\gamma \odot \mathbb{E}^{\prime} \leqslant s \gamma \odot \mathbb{E}^{\prime \prime}$ for every cardinal $\gamma$.)

11.6. Corollary. A nonempty class $\mathcal{A}$ is an ideal iff $\mathcal{A}$ satisfies the following three conditions:

(a) for every $\mathbb{A} \in \mathcal{C D D}_{N}$ and $\alpha \in \operatorname{Card}_{\infty}, \mathbb{A} \in \mathcal{A} \Longleftrightarrow \alpha \odot \mathbb{A} \in \mathcal{A}$,

(b) whenever $\left\{\mathbb{A}^{(s)}\right\}_{s \in S} \subset \mathcal{A}$ is a regular family of $N$-tuples such that $0<\operatorname{dim}(\mathbb{A}) \leqslant \aleph_{0}, \boxplus_{s \in S} \mathbb{A}^{(s)} \in \mathcal{A}$,

(c) $\mathbb{A} \leqslant s$ and $\mathbb{B} \in \mathcal{A}$ imply $\mathbb{A} \in \mathcal{A}$.

Proof. The necessity is clear. The sufficiency is in fact a consequence of Corollary 11.5. Indeed, if $\mathbb{A} \leqslant \mathbb{B}$ and $\mathbb{B} \in \mathcal{A}$, then $\alpha \odot \mathbb{A} \leqslant^{s} \alpha \odot \mathbb{B}$ for some infinite cardinal $\alpha$ (by Corollary 11.5). It follows from (a) that $\alpha \odot \mathbb{B} \in \mathcal{A}$ and consequently $\alpha \odot \mathbb{A} \in \mathcal{A}$ (by (c)) and $\mathbb{A} \in \mathcal{A}$, again by (a). Finally, if $\left\{\mathbb{A}^{(j)}\right\}_{j \in I} \subset \mathcal{A}$ and $\mathbb{A}=\bigoplus_{j \in I} \mathbb{A}^{(j)}$, then for huge enough $\alpha \in \operatorname{Card}_{\infty}$ one has $\alpha \odot \mathbb{A}^{(j)}=\alpha \odot \mathbb{E}^{(j)}$ with $\mathbb{E}^{(j)} \leqslant{ }^{s} \mathbb{J}(j \in I)$ and $\alpha \odot \mathbb{A}=\alpha \odot \mathbb{E}$ for some $\mathbb{E} \leqslant^{s} \mathbb{J}$ (see the proof of Corollary 11.5). Thanks 
to (a), $\mathbb{E}^{(j)} \in \mathcal{A}$ and it is enough to show that $\mathbb{E} \in \mathcal{A}$. We see that $\mathbb{E}^{(j)} \leqslant^{s} \mathbb{E}$ and $\mathbb{E}=\bigvee_{j \in I} \mathbb{E}^{(j)}$. These imply (compare with the proof of Theorem 6.1, page 13) that there is a regular family $\left\{\mathbb{B}^{(j)}\right\}_{j \in I}$ such that $\boxplus_{j \in I} \mathbb{B}^{(j)}=\mathbb{E}$ and $\mathbb{B}^{(j)} \leqslant s \mathbb{E}^{(j)}(j \in I)$. We infer from (c) that $\mathbb{B}^{(j)} \in \mathcal{A}$ for all $j \in I$. Now thanks to Proposition 9.10, each of $\mathbb{B}^{(j)}$ 's may be written in the form $\boxplus_{s \in S_{j}} \mathbb{A}^{(s, j)}$ with $0<\operatorname{dim}\left(\mathbb{A}^{(s, j)}\right) \leqslant \aleph_{0}$. Consequently, (c) yields $\mathbb{A}^{(s, j)} \in \mathcal{A}$ and hence $\mathbb{E} \in \mathcal{A}$ as well, by (b).

11.7. Example. Sometimes it may be useful to have the common partition of unity for several members of $\mathcal{C D} \mathcal{D}_{N}$ (in particular, to find the partition of unity induced by their direct sum). It may be understood as follows. For simplicity, we shall describe this idea only for two $\mathrm{N}$ tuples. Below we involve Proposition 6.4 (page 15) several times, with no comment.

Let $\mathbb{A}, \mathbb{B} \in \mathcal{C D} \mathcal{D}_{N}$. Let $\Upsilon^{2}=\{(i, \alpha, \beta):(i, \alpha),(i, \beta) \in \Upsilon\}$ and $\Upsilon_{*}^{2}=\left\{(i, \alpha, \beta):(i, \alpha),(i, \beta) \in \Upsilon_{*}\right\}$. For $(i, \alpha, \beta) \in \Upsilon^{2}$ let $\mathbb{E}_{\alpha, \beta}^{i}=$ $\mathbb{E}_{\alpha}^{i}(\mathbb{A}) \wedge \mathbb{E}_{\beta}^{i}(\mathbb{B})$. Additionally, we put $\mathbb{E}_{s m, \alpha}=\mathbb{E}_{s m}(\mathbb{A}) \wedge \mathbb{E}_{\alpha}^{I I}(\mathbb{B})$ and $\mathbb{E}_{\alpha, s m}=\mathbb{E}_{\alpha}^{I I}(\mathbb{A}) \wedge \mathbb{E}_{s m}(\mathbb{B})$ for $\alpha \in \Lambda_{I I}$. One may check that then $\mathbb{J}_{i}=$ $\boxplus_{\alpha, \beta \in \Lambda_{i}} \mathbb{E}_{\alpha, \beta}^{i}$ for $i \in\{I, I I, I I I\} ; \mathbb{E}_{\alpha, s m}$ and $\mathbb{E}_{s m, \alpha}$ are semiminimal and

$$
\mathbb{E}_{1, \alpha}^{I I}=\aleph_{0} \odot \mathbb{E}_{s m, \alpha}, \quad \mathbb{E}_{\alpha, 1}^{I I}=\aleph_{0} \odot \mathbb{E}_{\alpha, s m}
$$

for each $\alpha \in \Lambda_{I I}$. Further,

$$
\begin{aligned}
\mathbb{A}=\left(\bigoplus_{\alpha \in \operatorname{Card}_{\infty}} \mathbb{E}_{s m, \alpha}\right) & \boxplus\left(\bigoplus_{\alpha \in \operatorname{Card}_{\infty}} \alpha \odot \mathbb{E}_{\alpha, 1}^{I I}\right) \\
& \boxplus\left(\bigoplus_{(i, \alpha, \beta) \in \Upsilon_{*}^{2}} \alpha \odot \mathbb{E}_{\alpha, \beta}^{i}\right) \boxplus\left(\mathbb{E}_{s m, 1} \boxplus \mathbb{E}_{s m, 0}\right)
\end{aligned}
$$

and correspondingly

$$
\begin{aligned}
\mathbb{B}=\left(\bigoplus_{\alpha \in \operatorname{Card} \infty} \alpha \odot \mathbb{E}_{1, \alpha}^{I I}\right) \boxplus\left(\bigoplus_{\alpha \in \operatorname{Card} \infty} \mathbb{E}_{\alpha, s m}\right) \\
\\
\boxplus\left(\bigoplus_{(i, \alpha, \beta) \in \Upsilon_{*}^{2}} \beta \odot \mathbb{E}_{\alpha, \beta}^{i}\right) \boxplus\left(\mathbb{E}_{1, s m} \boxplus \mathbb{E}_{0, s m}\right) .
\end{aligned}
$$

In particular, thanks to (11-3),

$$
\mathbb{A} \oplus \mathbb{B}=\left[\mathbb{E}_{s m, 0} \boxplus \mathbb{E}_{0, s m} \boxplus\left(\mathbb{E}_{s m, 1} \oplus \mathbb{E}_{1, s m}\right)\right] \boxplus \bigoplus_{(i, \alpha, \beta) \in \Upsilon_{\#}^{2}}(\alpha+\beta) \odot \mathbb{E}_{\alpha, \beta}^{i}
$$

where $\Upsilon_{\#}^{2}=\Upsilon^{2} \backslash\{(I I, \alpha, \beta):(\alpha, \beta)=(0,1),(1,0),(1,1)\}$. So (below $\left.(i, \gamma) \in \Upsilon_{*}\right)$,

$$
\left\{\begin{array}{l}
\mathbb{E}_{s m}(\mathbb{A} \oplus \mathbb{B})=\mathbb{E}_{s m, 0} \boxplus \mathbb{E}_{0, s m} \boxplus\left[\mathbb{E}_{s m, 1} \oplus \mathbb{E}_{1, s m}\right], \\
\mathbb{E}_{1}^{I I}(\mathbb{A} \oplus \mathbb{B})=\mathbb{E}_{0,1}^{I I} \boxplus \mathbb{E}_{1,0}^{I I} \boxplus \mathbb{E}_{1,1}^{I I}, \\
\mathbb{E}_{\gamma}^{i}(\mathbb{A} \oplus \mathbb{B})=\bigoplus\left\{\mathbb{E}_{\alpha, \beta}^{i}:(i, \alpha, \beta) \in \Upsilon_{\#}^{2}, \alpha+\beta=\gamma\right\} .
\end{array}\right.
$$


In a similar manner one may find the formulas for $\mathbb{A} \vee \mathbb{B}$ and $\mathbb{A} \wedge \mathbb{B}$ and the partitions of unity induced by them.

\section{Algebraic and order properties of $\operatorname{eDD}_{N}$}

The following is a kind of folklore (see e.g. [19, Exercise 6.9.14]): if $p$ and $q$ are two projections in a von Neumann algebra $\mathcal{M}$ such that $n \odot p \sim n \odot q$ for some $n \geqslant 1$, then $p \sim q$. This has an interesting consequence for the class $\mathcal{C D D}_{N}$ :

$(\mathrm{AO} 1) n \odot \mathbb{A}=n \odot \mathbb{B} \Longrightarrow \mathbb{A}=\mathbb{B}$

provided $n$ is positive and finite. Further properties in this style are listed below.

(AO2) For finite positive $n$ and $m: n \odot \mathbb{A}=m \odot \mathbb{B} \Longleftrightarrow \mathbb{A}=k \odot \mathbb{X}$ and $\mathbb{B}=l \odot \mathbb{X}$ for some $\mathbb{X} \in \mathcal{C D} \mathcal{D}_{N}$ with $k=m / \operatorname{GCD}(n, m)$ and $l=n / \operatorname{GCD}(n, m)$ ('GCD' is the abbreviation for the greatest common divisor). If $n \neq m$, then $n \odot \mathbb{A}=m \odot \mathbb{A} \Longleftrightarrow \mathbb{A}=$ $\aleph_{0} \odot \mathbb{A}$.

(AO3) If $\alpha$ and $\beta$ are cardinals such that $\alpha<\beta$ and $\beta$ is infinite, then

$$
\alpha \odot \mathbb{A}=\beta \odot \mathbb{B} \Longleftrightarrow \mathbb{A}=\beta \odot \mathbb{B} \text {. }
$$

((AO2) and (AO3) follow from (11-1); cf. also the beginning of Section 14.)

(AO4) For a nontrivial $\mathbb{A} \in \mathcal{C D} \mathcal{D}_{N}$ the following conditions are equivalent:

(i) for any $\mathbb{X}, \mathbb{Y} \in \mathcal{C D} \mathcal{D}_{N}, \mathbb{A} \oplus \mathbb{X}=\mathbb{A} \oplus \mathbb{Y} \Longleftrightarrow \mathbb{X}=\mathbb{Y}$,

(ii) $\mathbb{B} \leqslant^{s} \mathbb{A}$ and $\mathbb{A} \oplus \mathbb{B}=\mathbb{A}$ imply $\mathbb{B}=\mathbb{O}$,

(iii) $\mathcal{W}^{\prime}(A)$ is finite,

(iv) $\mathbb{E}_{\alpha}^{i}(\mathbb{A})=\mathbb{O}$ for each $i \in\{I, I I, I I I\}$ and infinite $\alpha$.

All $N$-tuples $\mathbb{A}$ satisfying (i) form a set, denoted by $\mathcal{F J N}_{N}$. $\left(\mathcal{F J N}_{N}, \oplus\right)$ is a semigroup which may be enlarged to an Abelian group (by (i)).

(AO5) For every $\mathbb{A} \in \mathcal{F J N}_{N}$ and $\mathbb{B} \geqslant \mathbb{A}$ there is a unique $\mathbb{X}$ such that $\mathbb{A} \oplus \mathbb{X}=\mathbb{B}$. Thus, $\mathbb{B} \ominus \mathbb{A}$ is well defined in that case.

(AO6) Let $S$ be an infinite set whose power is a limit cardinal. For every collection $\left\{\mathbb{A}^{(s)}\right\}_{s \in S} \subset \mathcal{C D} \mathcal{D}_{N}$,

$$
\bigoplus_{s \in S} \mathbb{A}^{(s)}=\bigvee\left\{\bigoplus_{s \in S^{\prime}} \mathbb{A}^{(s)}: S^{\prime} \subset S, 0<\operatorname{card}\left(S^{\prime}\right)<\operatorname{card}(S)\right\}
$$

In particular, for every sequence $\left(\mathbb{B}^{(n)}\right)_{n=1}^{\infty} \subset \mathcal{C D} \mathcal{D}_{N}$,

$$
\bigoplus_{n=1}^{\infty} \mathbb{B}^{(n)}=\bigvee_{n=1}^{\infty} \mathbb{B}^{(1)} \oplus \ldots \oplus \mathbb{B}^{(n)}
$$

and for each $\mathbb{A} \in \mathcal{C} \mathcal{D} \mathcal{D}_{N}$ and an infinite limit cardinal $\gamma$,

$$
\gamma \odot \mathbb{A}=\bigvee_{\alpha<\gamma} \alpha \odot \mathbb{A} \text {. }
$$


(By Proposition 8.8, page 21.)

(AO7) Whenever $\mathbb{A} \leqslant \mathbb{B}$, there are $(\mathbb{B} \ominus \mathbb{A})^{\nabla},(\mathbb{B} \ominus \mathbb{A})_{\Delta} \in \mathcal{C D D}_{N}$ such that $\mathbb{A} \oplus \mathbb{X}=\mathbb{B}$ iff $(\mathbb{B} \ominus \mathbb{A})_{\Delta} \leqslant \mathbb{X} \leqslant(\mathbb{B} \ominus \mathbb{A})^{\nabla}$. Moreover, if $\mathbb{B}=2 \odot \mathbb{B}$, then $(\mathbb{B} \ominus \mathbb{A})_{\Delta} \leqslant \leqslant^{s} \mathbb{B}=(\mathbb{B} \ominus \mathbb{A})^{\nabla}$. $\mathbb{B} \ominus \mathbb{A}$ is well defined iff $(\mathbb{B} \ominus \mathbb{A})^{\nabla}=(\mathbb{B} \ominus \mathbb{A})_{\Delta}$. (See Theorem 8.7, page 20.)

(AO8) If $\mathbb{A} \leqslant s \mathbb{B}$, then $(\mathbb{B} \ominus \mathbb{A})_{\Delta}=\mathbb{B} \boxminus \mathbb{A}$. (Thanks to (PR1), page 9.) $(\mathrm{AO9})(\mathbb{B} \ominus \mathbb{A})_{\Delta} \leqslant(\mathbb{B} \ominus \mathbb{X})_{\Delta} \oplus(\mathbb{X} \ominus \mathbb{A})_{\Delta} \leqslant(\mathbb{B} \ominus \mathbb{X})^{\nabla} \oplus(\mathbb{X} \ominus \mathbb{A})^{\nabla} \leqslant$ $(\mathbb{B} \ominus \mathbb{A})^{\nabla}$ whenever $\mathbb{A} \leqslant \mathbb{X} \leqslant \mathbb{B}$.

$(\mathrm{AO} 10)(\mathbb{B} \ominus \mathbb{A})_{\Delta} \leqslant(\mathbb{B} \ominus \mathbb{A})^{\nabla}$ provided $\mathbb{A} \leqslant \mathbb{B}$.

Let us prove (AO10). We use the notation of Example 11.7. We infer from (11-4) and (11-5) that $\mathbb{A} \leqslant \mathbb{B}$ iff $\mathbb{E}_{s m, 1} \leqslant \mathbb{E}_{1, s m}, \mathbb{E}_{s m, 0}=\mathbb{O}$ and for every $\gamma \in \operatorname{Card}_{\infty}$ and $(i, \alpha, \beta) \in \Upsilon_{*}^{2}$ with $\alpha>\beta$,

$$
\mathbb{E}_{\gamma, 1}^{I I}=\mathbb{E}_{\alpha, \beta}^{i}=\mathbb{O} \text {. }
$$

In that case (11-4) reduces to

$$
\mathbb{A}=\left(\bigoplus_{\alpha \in \operatorname{Card}_{\infty}} \mathbb{E}_{s m, \alpha}\right) \boxplus\left(\prod_{\substack{(i, \alpha, \beta) \in \Upsilon_{*}^{2} \\ \alpha \leqslant \beta}} \alpha \odot \mathbb{E}_{\alpha, \beta}^{i}\right) \boxplus \mathbb{E}_{s m, 1},
$$

while (11-5) is equivalent to

$$
\mathbb{B}=\left(\bigoplus_{\alpha \in \operatorname{Card} \infty} \alpha \odot \mathbb{E}_{1, \alpha}^{I I}\right) \boxplus\left(\bigoplus_{\substack{(i, \alpha, \beta) \in \Upsilon_{*}^{2} \\ \alpha \leqslant \beta}} \beta \odot \mathbb{E}_{\alpha, \beta}^{i}\right) \boxplus \mathbb{E}_{s m}(\mathbb{B}) .
$$

Now we infer from the above formulas and (AO5) that

$$
\begin{aligned}
(\mathbb{B} \ominus \mathbb{A})_{\Delta}=\left[\mathbb{E}_{s m}(\mathbb{B}) \ominus \mathbb{E}_{s m, 1}\right] \boxplus\left(\bigoplus_{\alpha \in \operatorname{Card}_{\infty}} \alpha \odot \mathbb{E}_{1, \alpha}^{I I}\right) \\
\\
\boxplus\left(\bigoplus\left\{(\beta-\alpha) \odot \mathbb{E}_{\alpha, \beta}^{i}: \quad(i, \alpha, \beta) \in \Upsilon_{*}^{2}, \quad \alpha<\beta\right\}\right)
\end{aligned}
$$

where $\beta-\alpha=\beta$ provided $\beta$ is infinite (and $\beta>\alpha$ ). The above formula may be written in the following self-contained form:

$$
\begin{aligned}
(\mathbb{B} \ominus \mathbb{A})_{\Delta}=\left[\mathbb{E}_{s m}(\mathbb{B})\right. & \left.\ominus\left(\mathbb{E}_{s m}(\mathbb{A}) \wedge \mathbb{E}_{1}^{I I}(\mathbb{B})\right)\right] \\
& \boxplus\left[\bigoplus_{(i, \alpha, \beta) \in \Upsilon_{+}^{2}}(\beta-\alpha) \odot\left(\mathbb{E}_{\alpha}^{i}(\mathbb{A}) \wedge \mathbb{E}_{\beta}^{i}(\mathbb{B})\right)\right]
\end{aligned}
$$

where $\Upsilon_{+}^{2}=\left\{(i, \alpha, \beta) \in \Upsilon^{2}: \quad \alpha<\beta,(i, \alpha, \beta) \neq(I I, 0,1)\right\}$. It is also easy to verify that $(\mathbb{B} \ominus \mathbb{A})^{\nabla}=(\mathbb{B} \ominus \mathbb{A})_{\Delta} \oplus \mathbb{X}$ where $\mathbb{X}=\boxplus_{\alpha \in \operatorname{Card}_{\infty}} \alpha \odot$ $\left[\mathbb{E}_{\alpha, \alpha}^{I} \boxplus \mathbb{E}_{\alpha, \alpha}^{I I} \boxplus \mathbb{E}_{\alpha, \alpha}^{I I I}\right]$. Since $\mathbb{X} \perp_{u}(\mathbb{B} \ominus \mathbb{A})_{\Delta}$, the proof of $(\mathrm{AO} 10)$ is finished. Recall that we have shown that

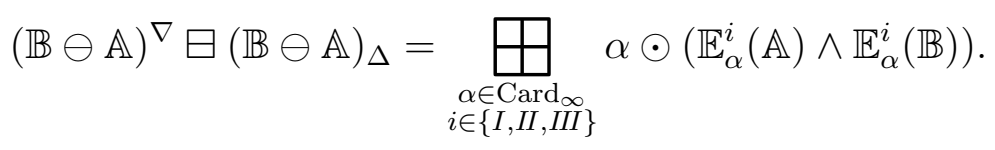


In particular, $(\mathbb{B} \ominus \mathbb{A})^{\nabla}=(\mathbb{B} \ominus \mathbb{A})_{\Delta}$ if and only if $\mathbb{E}_{\alpha}^{i}(\mathbb{A}) \perp_{u} \mathbb{E}_{\alpha}^{i}(\mathbb{B})$ for every infinite $\alpha$. This proves

(AO11) Whenever $\mathbb{A} \leqslant \mathbb{B}, B \ominus \mathbb{A}$ is well defined iff $\mathbb{E}_{\alpha}^{i}(\mathbb{A}) \perp_{u} \mathbb{E}_{\alpha}^{i}(\mathbb{B})$ for every $\alpha \in \operatorname{Card}_{\infty}$ and $i \in\{I, I I, I I I\}$.

$(\mathrm{AO} 12)(\mathbb{B} \ominus \mathbb{X})_{\Delta} \vee(\mathbb{X} \ominus \mathbb{A})_{\Delta} \leqslant(\mathbb{B} \ominus \mathbb{A})_{\Delta}$ whenever $\mathbb{A} \leqslant \mathbb{X} \leqslant \mathbb{B}$.

(AO13) For any nonempty set $\left\{\mathbb{A}^{(s)}\right\}_{s \in S} \subset \mathcal{C D} \mathcal{D}_{N}$ and $\mathbb{B} \in \mathcal{C D} \mathcal{D}_{N}$, $\mathbb{B} \vee\left(\bigwedge_{s \in S} \mathbb{A}^{(s)}\right)=\bigwedge_{s \in S}\left(\mathbb{B} \vee \mathbb{A}^{(s)}\right)$ and $\mathbb{B} \wedge\left(\bigvee_{s \in S} \mathbb{A}^{(s)}\right)=\bigvee_{s \in S}(\mathbb{B} \wedge$ $\left.\mathbb{A}^{(s)}\right)$.

(AO14) For any nonempty set $\left\{\mathbb{A}^{(s)}\right\}_{s \in S}$ of $N$-tuples, any $\mathbb{A}, \mathbb{B} \in \mathcal{C D} \mathcal{D}_{N}$ and each $\alpha \in$ Card,

$$
\begin{array}{ll}
\alpha \odot(\mathbb{A} \wedge \mathbb{B})=(\alpha \odot \mathbb{A}) \wedge(\alpha \odot \mathbb{B}), & \\
\alpha \odot\left(\bigwedge_{s \in S} \mathbb{A}^{(s)}\right)=\bigwedge_{s \in S}\left(\alpha \odot \mathbb{A}^{(s)}\right) \quad \text { if } \quad & \quad \forall \text { is finite or } \\
\alpha \odot\left(\bigvee_{s \in S} \mathbb{A}^{(s)}\right)=\bigvee_{s \in S}\left(\alpha \odot \mathbb{A}^{(s)}\right) . &
\end{array}
$$

For proofs of (AO12)-(AO14) see Corollary 15.3 (page 49), Theorem 15.10 (page 53) and Proposition 15.11 (page 54).

12.1. Example. Taking into account (AO14), it seems to be surprising that in general $\alpha \odot\left(\bigwedge_{s \in S} \mathbb{A}^{(s)}\right)$ differs from $\bigwedge_{s \in S}\left(\alpha \odot \mathbb{A}^{(s)}\right)$ for infinite cardinal $\alpha$, even if $S$ is countable. Let us give a short counterexample for this. Let $\alpha \geqslant \aleph_{0}$ and $\mathbb{X} \in \mathcal{S M}_{N}$ be nontrivial. There is a sequence $\left(\mathbb{A}^{(n)}\right)_{n=1}^{\infty}$ such that $n \odot \mathbb{A}^{(n)}=\mathbb{X}$ (see the beginning of Section 14). Then $\alpha \odot \mathbb{A}^{(n)}=\alpha \odot \mathbb{X} \neq \mathbb{O}$, while $\bigwedge_{n=1}^{\infty} \mathbb{A}^{(n)}=\mathbb{O}$.

(AO12) has an interesting consequence.

12.2. Proposition. Let $\mathcal{A}, \mathcal{B} \subset \mathcal{C D} \mathcal{D}_{N}$ be nonempty sets. Then $\bigvee(\mathcal{A} \oplus$ $\mathcal{B})=(\bigvee \mathcal{A}) \oplus(\bigvee \mathcal{B})$ and $\bigwedge(\mathcal{A} \oplus \mathcal{B})=(\bigwedge \mathcal{A}) \oplus(\bigwedge \mathcal{B})$ where $\mathcal{A} \oplus \mathcal{B}=$ $\{\mathbb{A} \oplus \mathbb{B}: \mathbb{A} \in \mathcal{A}, \mathbb{B} \in \mathcal{B}\}$.

Proof. Since the proof for the l.u.b.'s is much simpler, we shall show only the g.l.b.'s part. It is clear that $(\bigwedge \mathcal{A}) \oplus(\bigwedge \mathcal{B}) \leqslant \bigwedge(\mathcal{A} \oplus \mathcal{B})$. To see the converse inequality, assume that $\mathbb{X} \leqslant \mathbb{A} \oplus \mathbb{B}$ for any $\mathbb{A} \in \mathcal{A}$ and $\mathbb{B} \in \mathcal{B}$. Fix $\mathbb{B} \in \mathcal{B}$ and put $\mathbb{E}=\bigwedge(\mathcal{A} \oplus\{\mathbb{B}\})$. For each $\mathbb{A} \in \mathcal{A}$ we clearly have $\mathbb{A} \oplus \mathbb{B} \geqslant \mathbb{E} \geqslant \mathbb{B}$ and consequently, thanks to (AO12), $(\mathbb{E} \ominus \mathbb{B})_{\Delta} \leqslant[(\mathbb{A} \oplus \mathbb{B}) \ominus \mathbb{B}]_{\Delta} \leqslant \mathbb{A}$ where the last inequality follows from the definition of $[\ldots]_{\Delta}$. So, $(\mathbb{E} \ominus \mathbb{B})_{\Delta} \leqslant \bigwedge \mathcal{A}$ and therefore $\mathbb{E}=$ $(\mathbb{E} \ominus \mathbb{B})_{\Delta} \oplus \mathbb{B} \leqslant(\bigwedge \mathcal{A}) \oplus \mathbb{B}$. This shows that

$$
\bigwedge(\mathcal{A} \oplus\{\mathbb{B}\}) \leqslant(\bigwedge \mathcal{A}) \oplus \mathbb{B}
$$

which yields

$$
\begin{aligned}
\bigwedge(\mathcal{A} \oplus \mathcal{B})=\bigwedge_{\mathbb{B} \in \mathcal{B}}[\bigwedge(\mathcal{A} \oplus & \{\mathbb{B}\})] \leqslant \bigwedge_{\mathbb{B} \in \mathcal{B}}[(\bigwedge \mathcal{A}) \oplus \mathbb{B}]= \\
& =\bigwedge(\mathcal{B} \oplus\{\bigwedge \mathcal{A}\}) \leqslant(\bigwedge \mathcal{B}) \oplus(\bigwedge \mathcal{A})
\end{aligned}
$$

and we are done. 
12.3. Corollary. Let $\mathcal{A}_{1}, \mathcal{A}_{2}, \mathcal{A}_{3}, \ldots$ be nonempty sets of members of $\operatorname{CDD}_{N}$ and let $\mathcal{A}=\left\{\bigoplus_{n=1}^{\infty} \mathbb{A}^{(n)}: \mathbb{A}^{(n)} \in \mathcal{A}_{n}(n \geqslant 1)\right\}$. Then $\bigvee \mathcal{A}=$ $\bigoplus_{n=1}^{\infty}\left(\bigvee \mathcal{A}_{n}\right)$.

Proof. It is clear that $\bigvee \mathcal{A} \leqslant \bigoplus_{n=1}^{\infty}\left(\bigvee \mathcal{A}_{n}\right)$. Conversely, by (AO6), $\bigoplus_{n=1}^{\infty}\left(\bigvee \mathcal{A}_{n}\right)=\bigvee_{n \geqslant 1}\left[\left(\bigvee \mathcal{A}_{1}\right) \oplus \ldots \oplus\left(\bigvee \mathcal{A}_{n}\right)\right]$. Now by induction and Proposition 12.2, $\left(\bigvee \mathcal{A}_{1}\right) \oplus \ldots \oplus\left(\bigvee \mathcal{A}_{n}\right)=\bigvee\left(\mathcal{A}_{1} \oplus \ldots \oplus \mathcal{A}_{n}\right) \leqslant \bigvee \mathcal{A}$ and we are done.

In the next section we shall prove a counterpart of Corollary 12.3 for uncountable collections of sets of $N$-tuples (see Theorem 13.2, page 37).

12.4. Example. It may be seen surprising that the counterpart of Corollary 12.3 for infima fails to be true, even if each of $\mathcal{A}_{n}$ 's is a finite collection of minimal normal $N$-tuples. That is, in general $\bigwedge\left(\bigoplus_{n=1}^{\infty} \mathcal{A}_{n}\right)$ differs from $\bigoplus_{n=1}^{\infty}\left(\bigwedge \mathcal{A}_{n}\right)$ where $\bigoplus_{n=1}^{\infty} \mathcal{A}_{n}=\left\{\bigoplus_{n=1}^{\infty} \mathbb{A}^{(n)}: \mathbb{A}^{(n)} \in \mathcal{A}_{n}\right\}$. Let us justify this claim.

For every $u \in L^{\infty}([0,1])$ we shall write, for simplicity, $\boldsymbol{X}_{u}$ to denote the $N$-tuple $\left(M_{u}, \ldots, M_{u}\right)$ where $M_{u}$ is the multiplication operator by $u$ on $L^{2}([0,1])$. For each pair $(n, m)$ of naturals with $1 \leqslant m \leqslant n$ let $j_{n, m}$ be the characteristic function of $[0,1] \backslash[(m-1) / n, m / n]$. Additionally, let id $\in L^{\infty}([0,1])$ be the identity map on $[0,1]$. Put $\mathbb{A}_{n, m}=\mathbb{X}_{j_{n, m} \text { id }}$ and $\mathcal{A}_{n}=\left\{\mathbb{A}_{n, j}: j=1, \ldots, n\right\}$. Then $\mathcal{A}_{n} \subset \mathcal{M} \mathcal{F}_{N}$ (because $\mathcal{W}^{\prime}\left(X_{\text {id }}\right)=$ $\left.\left\{M_{u}: u \in L^{\infty}([0,1])\right\}\right)$ and $\bigwedge \mathcal{A}_{n}=\mathbb{O}$ for every $n \geqslant 1$. However, if $\left(m_{n}\right)_{n=1}^{\infty}$ is any sequence of natural numbers such that $1 \leqslant m_{n} \leqslant n$, then $\bigoplus_{n=1}^{\infty} \mathbb{A}_{n, m_{n}} \geqslant \bigvee_{n \geqslant 1} \mathbb{A}_{n, m_{n}}=\mathbb{X}_{\text {id }}$ (the latter equality holds true since $\bigcup_{n=1}^{\infty}\left([0,1] \backslash\left[\left(m_{n}-1\right) / n, m_{n} / n\right]\right)$ is of full Lebesgue measure in $[0,1])$. Consequently, $\bigwedge\left(\bigoplus_{n=1}^{\infty} \mathcal{A}_{n}\right) \geqslant \mathbb{X}_{\mathrm{id}} \neq \mathbb{O}=\bigoplus_{n=1}^{\infty}\left(\bigwedge \mathcal{A}_{n}\right)$.

One may deduce from Example 8.9 (page 21) that the assumption in (AO6) that the power of $S$ is a limit cardinal is essential (in the next section we shall discuss in details (12-1) for sets $S$ whose power is not limit). However, for semiminimal parts of $N$-tuples a stronger property (than in (AO6)) holds true in general (see below). For simplicity, for every set $S$ let us denote by $\mathcal{P}_{f}(S)$ and $\mathcal{P}_{\omega}(S)$ the families of all finite and, respectively, countable (finite or infinite) subsets of $S$.

12.5. Proposition. Let $S$ be an infinite set and $\left\{\mathbb{A}^{(s)}\right\}_{s \in S}$ be an arbitrary collection of $N$-tuples, $\mathbb{A}=\bigoplus_{s \in S} \mathbb{A}^{(s)}$ and

$$
\mathbb{A}^{\prime}=\bigvee\left\{\bigoplus_{s \in S_{0}} \mathbb{A}^{(s)}: S_{0} \in \mathcal{P}_{f}(S)\right\}
$$

Then $\mathbb{E}_{\text {sm }}(\mathbb{A})=\mathbb{E}_{\text {sm }}\left(\mathbb{A}^{\prime}\right)$ and $\mathbb{E}_{\alpha}^{i}(\mathbb{A})=\mathbb{E}_{\alpha}^{i}\left(\mathbb{A}^{\prime}\right)$ for each $(i, \alpha) \in \Upsilon$ with finite $\alpha$.

Proof. It is clear that $\mathbb{E}_{0}^{i}(\mathbb{A})=\mathbb{E}_{0}^{i}\left(\mathbb{A}^{\prime}\right)$ for $i \in\{I, I I, I I I\}$. Further, let us prove that

$$
\mathbb{E}_{1}^{I I}(\mathbb{A})=\mathbb{E}_{1}^{I I}\left(\mathbb{A}^{\prime}\right)
$$


Since $\mathbb{A}^{\prime} \leqslant \mathbb{A} \ll \mathbb{A}^{\prime}$, (11-2) (page 26) shows that $\mathbb{E}_{1}^{I I}(\mathbb{A}) \leqslant \mathbb{E}_{1}^{I I}\left(\mathbb{A}^{\prime}\right)$. Conversely, if $\mathbb{X}_{a}=\mathbb{E}\left(\mathbb{X} \mid \mathbb{E}_{1}^{I I}\left(\mathbb{A}^{\prime}\right)\right)$ for every $\mathbb{X} \in \mathcal{C D} \mathcal{D}_{N}$, then $\left(\mathbb{A}^{\prime}\right)_{a}=$ $\bigvee\left\{\bigoplus_{s \in S_{0}}\left(\mathbb{A}^{(s)}\right)_{a}: \quad S_{0} \in \mathcal{P}_{f}(S)\right\}$ and $\mathbb{A}_{a}=\bigoplus_{s \in S}\left(\mathbb{A}^{(s)}\right)_{a}$. But $\left(\mathbb{A}^{\prime}\right)_{a}=$ $\mathbb{E}_{s m}\left(\mathbb{A}^{\prime}\right) \in \mathcal{S} \mathcal{M}_{N}$ and hence $\left(\mathbb{A}^{\prime}\right)_{a}=\mathbb{A}_{a}$, thanks to Proposition 12.6 (see below). So, $\mathbb{A}_{a} \in \mathcal{S M}_{N}$ and consequently, again by (11-2), $\mathbb{E}_{1}^{I I}\left(\mathbb{A}^{\prime}\right) \leqslant$ $\mathbb{E}_{1}^{I I}(\mathbb{A})$. This proves $(12-4)$.

Now we have $\mathbb{E}_{s m}(\mathbb{A})=\mathbb{E}\left(\mathbb{A} \mid \mathbb{E}_{1}^{I I}(\mathbb{A})\right)=\mathbb{A}_{a}=\left(\mathbb{A}^{\prime}\right)_{a}=\mathbb{E}_{s m}\left(\mathbb{A}^{\prime}\right)$.

It remains to check that $\mathbb{E}_{n}^{I}(\mathbb{A})=\mathbb{E}_{n}^{I}\left(\mathbb{A}^{\prime}\right)$ for natural $n$. Let $\mathbb{F}=$ $\boxplus_{n=1}^{\infty} \mathbb{E}_{n}^{I}(\mathbb{A})$ and $\mathbb{F}^{\prime}=\boxplus_{n=1}^{\infty} \mathbb{E}_{n}^{I}\left(\mathbb{A}^{\prime}\right)$. It is enough to show that $\mathbb{F}=\mathbb{F}^{\prime}$ which we leave for the reader (as it is similar to the proof of (12-4)).

The following result is in the same spirit.

12.6. Proposition. Let $S$ be an infinite set, $\left\{\mathbb{A}^{(s)}\right\}_{s \in S} \subset \mathcal{C D D} \mathcal{D}_{N}$ and let

$$
\mathbb{A}=\bigvee\left\{\bigoplus_{s \in S_{0}} \mathbb{A}^{(s)}: S_{0} \in \mathcal{P}_{f}(S)\right\}
$$

If $\mathbb{A} \in \mathcal{F J N}_{N}$, then $\mathbb{A}=\bigoplus_{s \in S} \mathbb{A}^{(s)}$.

Proof. Let $\mathcal{M}=\mathcal{W}^{\prime}(\boldsymbol{A})$ and $p_{s} \in E(\mathcal{M})(s \in S)$ correspond to $\mathbb{A}^{(s)}$ (by Proposition 4.1, page 9 ). Further, let tr: $\mathcal{M} \rightarrow \mathcal{Z}(\mathcal{M})$ be the trace on $\mathcal{M}$. For every $s \in S$ put $w_{s}=\operatorname{tr}\left(p_{s}\right)$. Since $\bigoplus_{s \in S_{0}} \mathbb{A}^{(s)} \leqslant \mathbb{A}$ where $S_{0} \in \mathcal{P}_{f}(S), \sum_{s \in S_{0}} w_{s} \leqslant 1$ and consequently $\sum_{s \in S} w_{s}$ is convergent and the sum is no greater than 1 . Recall that for any $q, q^{\prime} \in E(\mathcal{M})$, $q \preccurlyeq q^{\prime} \Longleftrightarrow \operatorname{tr}(q) \leqslant \operatorname{tr}\left(q^{\prime}\right)$ (see e.g. [34, Corollary 5.2.8] or [19, Theorem 8.4.3]). This implies that it is possible, well ordering the set $S$ and using transfinite induction, to construct a family $\left\{q_{s}\right\}_{s \in S}$ of mutually orthogonal projections in $\mathcal{M}$ such that $p_{s} \sim q_{s}$ for any $s \in S$. Hence $\sum_{s \in S} q_{s} \leqslant 1$ which yields $\bigoplus_{s \in S} \mathbb{A}^{(s)} \leqslant \mathbb{A}$ and we are done.

\section{ReCONSTRUCTING INFINITE OPERATIONS}

Classical algebraic structures deal with operations on pairs (such as the action of a semigroup). However, some operations naturally make sense also for infinitely (possibly uncountably) many arguments (e.g. unions of sets) and sometimes it is necessary to use these extended 'infinite' operations in order to understand, formulate or prove some statements. Unless infinite operations can be 'defined' (or characterized) in terms of their finite versions, every such a theorem may be seen as a result from outside the theory. The most typical example of an infinite operation is the union of a family of sets. However, it may be characterized by means of the union of two sets. Namely, for any family $\mathcal{A}$ put $\mathcal{A}^{\Delta}=\{B: A \cup B=B$ for each $A \in \mathcal{A}\}$ and then $\bigcup \mathcal{A}$ is the unique set $B \in \mathcal{A}^{\Delta}$ such that $B \cup C=C$ for any $C \in \mathcal{A}^{\Delta}$. This characterization is possible for a one simple reason: the union coincides with the l.u.b. of the family with respect to the inclusion order which may be defined in terms of the union 
of a pair. When we pass to the class $\mathcal{C D} \mathcal{D}_{N}$, the direct sum operation cannot be characterized in a similar manner, because $\bigoplus_{s \in S} \mathbb{A}^{(s)}$ differs, in general, from $\bigvee\left\{\bigoplus_{s \in S_{0}} \mathbb{A}^{(s)}: S_{0}\right.$ a finite subset of $\left.S\right\}$. Nevertheless, infinite direct sums may be reconstructed from the finite ones, and this is the subject of this section. Thus, every result of the paper concerning unitary equivalence classes of $N$-tuples is a part of the theory which starts with the class $\mathcal{C} \mathcal{D D}_{N}$ and the operation $\mathcal{C D D}_{N} \times \mathcal{C D D}_{N} \ni(\mathbb{A}, \mathbb{B}) \mapsto \mathbb{A} \oplus \mathbb{B} \in \mathcal{C D D}_{N}$. (This refers to the material of Sections $1-17$, but does not to the rest.)

Our aim is to show that $\bigoplus_{s \in S} \mathbb{A}^{(s)}$ may be 'recognized' if the only admissible 'tool' in the class $\mathcal{C} \mathcal{D D}_{N}$ is the direct sum operation of a pair. Below we draw step by step how to do this. Each of the listed steps begins with the tool which may be defined.

(ST1) ' $\mathbb{O}$ ': It is the unique member $\mathbb{A}$ of $\mathcal{C D} \mathcal{D}_{N}$ such that $\mathbb{A} \oplus \mathbb{X}=\mathbb{X}$ for every $\mathbb{X} \in \mathcal{C D} \mathcal{D}_{N}$.

(ST2) ' $\leqslant$ ': $\mathbb{A} \leqslant \mathbb{B}$ iff $\mathbb{B}=\mathbb{A} \oplus \mathbb{X}$ for some $\mathbb{X} \in \operatorname{eDD}_{N}$. Accordingly, the l.u.b.'s and g.l.b.'s are well defined.

(ST3) ' $\perp_{u}$ ': $\mathbb{A} \perp_{u} \mathbb{B} \Longleftrightarrow \mathbb{A} \wedge \mathbb{B}=\mathbb{O}$.

(ST4) ' $\leqslant^{s}: \mathbb{A} \leqslant^{s} \mathbb{B}$ iff $\mathbb{B}=\mathbb{A} \oplus \mathbb{X}$ for some $\mathbb{X}$ such that $\mathbb{X} \perp_{u} \mathbb{A}$.

(ST5) ' $⿴ 囗 十$ ' and ' $\boxminus$ ': $\mathbb{A}=\bigoplus_{s \in S} \mathbb{A}^{(s)}$ (S any set) iff $\mathbb{A}^{(s)} \perp_{u} \mathbb{A}^{\left(s^{\prime}\right)}$ for distinct $s, s^{\prime} \in S$ and $\mathbb{A}=\bigvee_{s \in S} \mathbb{A}^{(s)}$; if $\mathbb{A} \leqslant^{s} \mathbb{B}, \mathbb{B} \boxminus \mathbb{A}$ is the unique $\mathbb{X}$ such that $\mathbb{X} \perp_{u} \mathbb{A}$ and $\mathbb{B}=\mathbb{X} \oplus \mathbb{A}$.

(ST6) ' $\bigoplus_{n=1}^{\infty}$ ': $\bigoplus_{n=1}^{\infty} \mathbb{A}^{(n)}=\bigvee_{n \geqslant 1} \mathbb{A}^{(1)} \oplus \ldots \oplus \mathbb{A}^{(n)}$. In particular, $\aleph_{0} \odot \mathbb{A}$ is well defined for each $\mathbb{A}$.

(ST7) ' $\ll$ ': $\mathbb{A} \ll \mathbb{B}$ iff there is no $\mathbb{X} \neq \mathbb{O}$ such that $\mathbb{X} \leqslant \mathbb{A}$ and $\mathbb{X} \perp_{u} \mathbb{B}$.

(ST8) ' $\mathbb{E}(\mathbb{A} \mid \mathbb{B})^{\prime}: \mathbb{X}=\mathbb{E}(\mathbb{A} \mid \mathbb{B}) \Longleftrightarrow \exists \mathbb{Y}: \mathbb{A}=\mathbb{X} \oplus \mathbb{Y}, \mathbb{X} \ll \mathbb{B}$ and $\mathbb{Y} \perp_{u} \mathbb{B}$.

(ST9) 'Multiplicity free $N$-tuples': $\mathbb{A} \in \mathcal{M F}_{N}$ if and only if there is no $\mathbb{X} \neq \mathbb{O}$ such that $\mathbb{X} \oplus \mathbb{X} \leqslant \mathbb{A}$. Accordingly, $\mathbb{J}_{I}$ is well defined.

(ST10) 'Minimal $N$-tuples': $\mathbb{A}$ is minimal iff $\mathbb{A} \leqslant \mathbb{X}$ whenever $\mathbb{A} \ll \mathbb{X}$.

(ST11) 'Hereditary idempotents': $\mathbb{A} \in \mathcal{H J}_{N} \Longleftrightarrow \mathbb{B}=\mathbb{B} \oplus \mathbb{B}$ for each

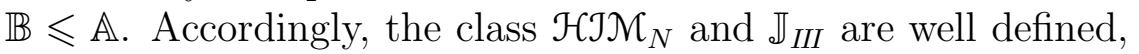
thanks to (ST10).

(ST12) 'Semiminimal $N$-tuples': Use (ST5), (ST7) and the definition of semiminimality.

(ST13) ' $\mathbb{J}_{I I}$ ': $\mathbb{J}_{I I}=\bigvee\left\{\aleph_{0} \odot \mathbb{X}: \mathbb{X} \in \mathcal{S} \mathcal{M}_{N}\right\}$ (use (ST6) and (ST12)).

(ST14) ' $\alpha \odot \mathbb{A}$ for $\mathbb{A} \leqslant \mathbb{J}_{i}$ ' with $i \in\{I, I I, I I I\}$ : Thanks to (ST6), we may assume that $\alpha>\aleph_{0}$. If $i=I I, \alpha \odot \mathbb{A}=\alpha \odot\left(\aleph_{0} \odot \mathbb{A}\right)$ and $\aleph_{0} \odot \mathbb{A} \leqslant^{s} \mathbb{J}_{I I}$; while for $i \neq I I$ one has $\mathbb{A} \leqslant^{s} \mathbb{J}_{i}$. These notices show that we may assume that $\mathbb{A} \leqslant^{s} \mathbb{J}_{i}$. Under this assumption, $\alpha \odot \mathbb{A}$ may be characterized by means of transfinite induction with respect to $\alpha$. When $\alpha$ is limit, it suffices to apply (AO6) (page 29). On the other hand, if $\alpha=\beta^{+}$and $\mathbb{A} \neq \mathbb{O}$,

$$
\alpha \odot \mathbb{A}=\bigwedge\left\{\mathbb{X} \mid \quad \forall \mathbb{B} \leqslant^{s} \mathbb{A}, \mathbb{B} \neq \mathbb{O}: \beta \odot \mathbb{B} \supsetneqq \mathbb{E}(\mathbb{X} \mid \mathbb{B})\right\}
$$


(This formula may be deduced from Theorem 11.1, page 26.)

$(\mathrm{ST} 15)$ ' $\mathbb{E}_{\alpha}^{i}(\mathbb{A})$ and $\mathbb{E}_{s m}(\mathbb{A})^{\prime}$ ': Thanks to Theorem 11.1 and previous steps.

(ST16) ' $\alpha \odot \mathbb{A}$ ' (arbitrary $\mathbb{A})$ : Use (ST15), Theorem 11.1, (ST14) and (ST5).

$(\operatorname{ST} 17) ' \aleph_{0} \cdot \operatorname{dim}(\mathbb{A}) '$ : Since

$$
\begin{aligned}
\aleph_{0} \cdot \operatorname{dim}(\mathbb{A})=\sum_{n=1}^{\infty} \aleph_{0} \cdot \operatorname{dim}\left(\mathbb{E}_{n}^{I}(\mathbb{A})\right)+ & \aleph_{0} \cdot \operatorname{dim}\left(\mathbb{E}_{1}^{I I}(\mathbb{A})\right) \\
& +\sum_{\substack{\alpha \in \operatorname{Card}_{\infty} \\
i \in\{I, I I, I I I\}}} \alpha\left[\aleph_{0} \cdot \operatorname{dim}\left(\mathbb{E}_{\alpha}^{i}(\mathbb{A})\right)\right]
\end{aligned}
$$

(cf. Theorem 11.1; $\operatorname{dim}\left(\mathbb{E}_{\text {sm }}(\mathbb{A})\right)=\operatorname{dim}\left(\mathbb{E}_{1}^{I I}(\mathbb{A})\right)$ ), it suffices to characterize the cardinal number $\aleph_{0} \cdot \operatorname{dim}(\mathbb{A})$ for $\mathbb{A} \leqslant^{s} \mathbb{J}$. But in that case it is quite easy, thanks to Proposition 9.10 (page 24$): \aleph_{0} \cdot \operatorname{dim}(\mathbb{A})$ (provided $\mathbb{A} \leqslant^{s} \mathbb{J}$ is nontrivial) is the least infinite cardinal number $\alpha$ such that each regular subfamily of $\left\{\mathbb{X}: \mathbb{O} \neq \mathbb{X} \leqslant^{s} \mathbb{A}\right\}$ has power no greater than $\alpha$.

Now we are ready to characterize infinite direct sums. For simplicity, let us put $\operatorname{Dim}(\mathbb{F})=\aleph_{0} \cdot \operatorname{dim}(\mathbb{F}), \mathbb{E}_{f}(\mathbb{F})=\mathbb{E}_{1}^{I I}(\mathbb{F}) \boxplus \boxplus_{n=1}^{\infty} \mathbb{E}_{n}^{I}(\mathbb{F})$ and $\mathbb{E}_{\alpha}(\mathbb{F})=\mathbb{E}_{\alpha}^{I}(\mathbb{F}) \boxplus \mathbb{E}_{\alpha}^{I I}(\mathbb{F}) \boxplus \mathbb{E}_{\alpha}^{I I I}(\mathbb{F})$ for $\alpha \in\{0\} \cup$ Card $_{\infty}$ and any $\mathbb{F} \in \mathcal{C D D}_{N}$. By (ST17) and (ST15), 'Dim', ' $\mathbb{E}_{f}$ ' and ' $\mathbb{E}_{\alpha}$ ' are well defined. Fix a collection $\left\{\mathbb{A}^{(s)}\right\}_{s \in S}$ of $N$-tuples and put $\mathbb{A}=\bigoplus_{s \in S} \mathbb{A}^{(s)}$ and $\mathbb{A}_{f}=\bigvee\left\{\bigoplus_{s \in S^{\prime}} \mathbb{A}^{(s)}: \quad S^{\prime} \in \mathcal{P}_{f}(S)\right\} . \quad \mathbb{A}_{f}$ is 'known' by (ST2). Thanks to (ST16) and (ST5), it suffices to find $\mathbb{E}_{s m}(\mathbb{A})$ and $\mathbb{E}_{\alpha}^{i}(\mathbb{A})$. By Proposition 12.5, $\mathbb{E}_{\alpha}^{i}(\mathbb{A})=\mathbb{E}_{\alpha}^{i}\left(\mathbb{A}^{\prime}\right)$ for $(i, \alpha) \in \Upsilon$ with finite $\alpha$ and $\mathbb{E}_{s m}(\mathbb{A})=\mathbb{E}_{s m}\left(\mathbb{A}^{\prime}\right)$. Since $\mathbb{E}_{\alpha}^{i}(\mathbb{A})=\mathbb{E}_{\alpha}(\mathbb{A}) \wedge \mathbb{J}_{i}$, we see that it remains to find $\mathbb{E}_{\alpha}(\mathbb{A})$ for infinite $\alpha$ (thanks to (ST9), (ST11) and (ST13)). To this end, let us show that

$$
\mathbb{E}_{\alpha}(\mathbb{A})=\bigvee x
$$

where

$$
\begin{aligned}
& X=\left\{\mathbb{X} \leqslant^{s} \mathbb{J} \boxminus\right.\left(\mathbb{E}_{0}(\mathbb{A}) \boxplus \mathbb{E}_{f}(\mathbb{A})\right) \mid \\
&\left.\forall \mathbb{Y} \leqslant s \mathbb{X}, \operatorname{Dim}(\mathbb{Y})=\aleph_{0}: \sum_{s \in S} \operatorname{Dim} \mathbb{E}\left(\mathbb{A}^{(s)} \mid \mathbb{Y}\right)=\alpha\right\} .
\end{aligned}
$$

It is clear that $\left.\mathbb{E}_{\alpha}(\mathbb{A}) \leqslant s\right] \boxminus\left(\mathbb{E}_{0}(\mathbb{A}) \boxplus \mathbb{E}_{f}(\mathbb{A})\right)$. Furthermore, if $\mathbb{Y} \leqslant^{s}$ $\mathbb{E}_{\alpha}(\mathbb{A})$, then $\bigoplus_{s \in S} \mathbb{E}\left(\mathbb{A}^{(s)} \mid \mathbb{Y}\right)=\mathbb{E}(\mathbb{A} \mid \mathbb{Y})=\alpha \odot \mathbb{Y}$ and thus

$$
\aleph_{0} \cdot \sum_{s \in S} \operatorname{dim}\left(\mathbb{E}\left(\mathbb{A}^{(s)} \mid \mathbb{Y}\right)\right)=\alpha
$$

provided $\operatorname{Dim}(\mathbb{Y})=\aleph_{0}$. This yields $\mathbb{E}_{\alpha}(\mathbb{A}) \in X$. Consequently, the proof of (13-1) will be finished if we show that $\mathbb{X} \leqslant \mathbb{E}_{\alpha}(\mathbb{A})$ for every $\mathbb{X} \in X$. To get this inequality, it is enough to check that $\mathbb{X} \perp_{u} \mathbb{E}_{\beta}(\mathbb{A})$ 
for any infinite $\beta \neq \alpha$. Suppose $\mathbb{Y}^{\prime}=\mathbb{X} \wedge \mathbb{E}_{\beta}(\mathbb{A})$ is nontrivial. Since $\mathbb{Y}^{\prime} \leqslant{ }^{s} \mathbb{J}$, we infer from Proposition 9.10 (page 24) that there is $\mathbb{Y} \leqslant \leqslant^{s} \mathbb{Y}^{\prime}$ with $\operatorname{Dim}(\mathbb{Y})=\aleph_{0}$. But then $\mathbb{Y} \leqslant^{s} \mathbb{X}$ and $\mathbb{E}(\mathbb{A} \mid \mathbb{Y})=\beta \odot \mathbb{Y}$ (because $\left.\mathbb{Y} \leqslant \mathbb{E}_{\beta}(\mathbb{A})\right)$. Consequently, $\aleph_{0} \cdot \sum_{s \in S} \operatorname{dim}\left(\mathbb{E}\left(\mathbb{A}^{(s)} \mid \mathbb{Y}\right)\right)=\beta$ which denies the fact that $\mathbb{X} \in \mathcal{X}$ and finishes the proof of (13-1).

The arguments of this section prove

13.1. Proposition. If $\Phi: \operatorname{CDD}_{N} \rightarrow \mathcal{C D D}_{N}$ is a bijective assignment such that $\Phi(\mathbb{A} \oplus \mathbb{B})=\Phi(\mathbb{A}) \oplus \Phi(\mathbb{B})$ for any $\mathbb{A}, \mathbb{B} \in \mathcal{C D D}_{N}$, then $\Phi$ preserves all notions, features and operations appearing in (ST1)-(ST17) and

$$
\Phi\left(\bigoplus_{s \in S} \mathbb{A}^{(s)}\right)=\bigoplus_{s \in S} \Phi\left(\mathbb{A}^{(s)}\right)
$$

for any set $\left\{\mathbb{A}^{(s)}\right\}_{s \in S} \subset \mathcal{C D} \mathcal{D}_{N}$.

Let us now discuss the relation between both sides of (12-1) for a set $S$ whose cardinality is not limit, i.e. $\operatorname{card}(S)=\gamma^{+}$for some infinite cardinal $\gamma$. Let $\mathbb{A}^{\prime}=\bigvee\left\{\bigoplus_{s \in S^{\prime}} \mathbb{A}^{(s)}: S^{\prime} \subset S, 0<\operatorname{card}\left(S^{\prime}\right) \leqslant \gamma\right\}$. By Proposition $12.5, \mathbb{E}_{s m}(\mathbb{A})=\mathbb{E}_{s m}\left(\mathbb{A}^{\prime}\right)$ and $\mathbb{E}_{\alpha}^{i}(\mathbb{A})=\mathbb{E}_{\alpha}^{i}\left(\mathbb{A}^{\prime}\right)$ for every $(i, \alpha) \in \Upsilon$ with finite $\alpha$. These equalities are more general: we claim that

$$
\mathbb{E}_{\alpha}^{i}(\mathbb{A})=\mathbb{E}_{\alpha}^{i}\left(\mathbb{A}^{\prime}\right)
$$

provided $\alpha \neq \gamma, \gamma^{+}$. To show this, it suffices to check that $\mathbb{E}_{\alpha}^{i}\left(\mathbb{A}^{\prime}\right) \leqslant$ $\mathbb{E}_{\alpha}^{i}(\mathbb{A})$ for $\alpha \neq \gamma, \gamma^{+}$and $\mathbb{E}_{\gamma}^{i}\left(\mathbb{A}^{\prime}\right) \boxplus \mathbb{E}_{\gamma^{+}}^{i}\left(\mathbb{A}^{\prime}\right) \leqslant \mathbb{E}_{\gamma}^{i}(\mathbb{A}) \boxplus \mathbb{E}_{\gamma^{+}}^{i}(\mathbb{A})$ (since we deal with partitions of unity). We need to check only infinite $\alpha$ 's.

First assume $\aleph_{0} \leqslant \alpha<\gamma$. Fix $\mathbb{X} \leqslant \mathbb{E}_{\alpha}^{i}\left(\mathbb{A}^{\prime}\right)$ with $\operatorname{Dim}(\mathbb{X})=\aleph_{0}$. Let

$$
S^{\prime}=\left\{s \in S: \mathbb{E}\left(\mathbb{A}^{(s)} \mid \mathbb{X}\right) \neq \mathbb{O}\right\} .
$$

If the power of $S^{\prime}$ was greater than $\alpha$, there would exist a subset $S^{\prime \prime}$ of $S^{\prime}$ with $\operatorname{card}\left(S^{\prime \prime}\right)=\alpha^{+} \leqslant \gamma$. Then we would have $\bigoplus_{s \in S^{\prime \prime}} \mathbb{A}^{(s)} \leqslant \mathbb{A}^{\prime}$ and $\alpha \odot \mathbb{X}=\mathbb{E}\left(\mathbb{A}^{\prime} \mid \mathbb{X}\right) \geqslant \bigoplus_{s \in S^{\prime \prime}} \mathbb{E}\left(\mathbb{A}^{(s)} \mid \mathbb{X}\right)$ which denies the fact that $\operatorname{Dim}(\alpha \odot \mathbb{X})=\alpha<\operatorname{card}\left(S^{\prime \prime}\right) \leqslant \operatorname{Dim}\left(\bigoplus_{s \in S^{\prime \prime}} \mathbb{E}\left(\mathbb{A}^{(s)} \mid \mathbb{X}\right)\right)$. We infer from this that $\operatorname{card}\left(S^{\prime}\right) \leqslant \alpha$. So, $\mathbb{E}(\mathbb{A} \mid \mathbb{X})=\mathbb{E}\left(\mathbb{A}^{\prime} \mid \mathbb{X}\right)$. Consequently, $\mathbb{E}\left(\mathbb{A} \mid \mathbb{E}_{\alpha}^{i}\left(\mathbb{A}^{\prime}\right)\right)=\mathbb{E}\left(\mathbb{A}^{\prime} \mid \mathbb{E}_{\alpha}^{i}\left(\mathbb{A}^{\prime}\right)\right)=\alpha \odot \mathbb{E}_{\alpha}^{i}\left(\mathbb{A}^{\prime}\right)$ (thanks to Proposition 9.10, page 24$)$. This means that $\mathbb{E}_{\alpha}^{i}\left(\mathbb{A}^{\prime}\right) \leqslant \mathbb{E}_{\alpha}^{i}(\mathbb{A})$.

Now assume that $\alpha \geqslant \gamma^{+}=\operatorname{card}(S)$. As before, take $\mathbb{X} \leqslant \mathbb{E}_{\alpha}^{i}\left(\mathbb{A}^{\prime}\right)$ with $\operatorname{Dim}(\mathbb{X})=\aleph_{0}$. Then $\alpha \odot \mathbb{X}=\mathbb{E}\left(\mathbb{A}^{\prime} \mid \mathbb{X}\right) \geqslant \mathbb{E}\left(\mathbb{A}^{(s)} \mid \mathbb{X}\right)$ for every $s \in S$ and thus $\mathbb{E}(\mathbb{A} \mid \mathbb{X})=\bigoplus_{s \in S} \mathbb{E}\left(\mathbb{A}^{(s)} \mid \mathbb{X}\right) \leqslant \operatorname{card}(S) \odot(\alpha \odot \mathbb{X})=$ $\alpha \odot \mathbb{X}=\mathbb{E}\left(\mathbb{A}^{\prime} \mid \mathbb{X}\right) \leqslant \mathbb{E}(\mathbb{A} \mid \mathbb{X})$. So, $\mathbb{E}(\mathbb{A} \mid \mathbb{X})=\mathbb{E}\left(\mathbb{A}^{\prime} \mid \mathbb{X}\right)$ and consequently $\mathbb{E}_{\alpha}^{i}\left(\mathbb{A}^{\prime}\right) \leqslant \mathbb{E}_{\alpha}^{i}(\mathbb{A})$.

To finish the proof of (13-2), it remains to consider $\alpha=\gamma$. For $\mathbb{X}=$ $\mathbb{E}_{\gamma}^{i}\left(\mathbb{A}^{\prime}\right)$ we readily have $\gamma \odot \mathbb{X}=\mathbb{E}\left(\mathbb{A}^{\prime} \mid \mathbb{X}\right) \leqslant \mathbb{E}(\mathbb{A} \mid \mathbb{X})=\bigoplus_{s \in S} \mathbb{E}\left(\mathbb{A}^{(s)} \mid \mathbb{X}\right) \leqslant$ $\operatorname{card}(S) \odot \mathbb{E}\left(\mathbb{A}^{\prime} \mid \mathbb{X}\right)=\gamma^{+} \odot \mathbb{X}$. These inequalities imply that $\mathbb{X} \leqslant$ $\mathbb{E}_{\gamma}^{i}(\mathbb{A}) \boxplus \mathbb{E}_{\gamma^{+}}^{i}(\mathbb{A})$ and we are done. 
Having (13-2), we obtain that $\mathbb{E}_{\gamma}^{i}\left(\mathbb{A}^{\prime}\right) \boxplus \mathbb{E}_{\gamma^{+}}^{i}\left(\mathbb{A}^{\prime}\right)=\mathbb{E}_{\gamma}^{i}(\mathbb{A}) \boxplus \mathbb{E}_{\gamma^{+}}^{i}(\mathbb{A})$. We also know that $\mathbb{E}_{\gamma^{+}}^{i}\left(\mathbb{A}^{\prime}\right) \leqslant^{s} \mathbb{E}_{\gamma^{+}}^{i}(\mathbb{A})$ (by the above argument and Theorem 6.1, page 13). So, $\mathbb{E}_{\gamma^{+}}^{i}(\mathbb{A}) \boxminus \mathbb{E}_{\gamma^{+}}^{i}\left(\mathbb{A}^{\prime}\right)$ gives full information about the difference between $\mathbb{A}$ and $\mathbb{A}^{\prime}$. We have

$$
\begin{aligned}
\mathbb{E}_{\gamma^{+}}^{i}(\mathbb{A}) \boxminus \mathbb{E}_{\gamma^{+}}^{i}\left(\mathbb{A}^{\prime}\right)= & \bigvee\left\{\mathbb{Y} \leqslant^{s} \mathbb{E}_{\gamma}^{i}\left(\mathbb{A}^{\prime}\right) \mid \quad \forall \mathbb{X} \leqslant s \mathbb{Y}, \mathbb{X} \neq \mathbb{O}:\right. \\
& \left.\operatorname{card}\left(\left\{s \in S: \mathbb{E}\left(\mathbb{A}^{(s)} \mid \mathbb{X}\right) \neq \mathbb{O}\right\}\right)=\gamma^{+}\right\}
\end{aligned}
$$

Indeed, $\mathbb{E}_{\gamma^{+}}^{i}(\mathbb{A}) \boxminus \mathbb{E}_{\gamma^{+}}^{i}\left(\mathbb{A}^{\prime}\right) \leqslant \mathbb{E}_{\gamma}^{i}\left(\mathbb{A}^{\prime}\right)$ and if $\mathbb{X} \leqslant \mathbb{E}_{\gamma^{+}}^{i}(\mathbb{A}) \boxminus \mathbb{E}_{\gamma^{+}}^{i}\left(\mathbb{A}^{\prime}\right)$ is nontrivial, then there is $\mathbb{X}^{\prime} \leqslant^{s} \mathbb{X}$ with $\operatorname{Dim}\left(\mathbb{X}^{\prime}\right)=\aleph_{0}$ (by Proposition 9.10). Then $\bigoplus_{s \in S} \mathbb{E}\left(\mathbb{A}^{(s)} \mid \mathbb{X}^{\prime}\right)=\mathbb{E}\left(\mathbb{A} \mid \mathbb{X}^{\prime}\right)=\gamma^{+} \odot \mathbb{X}^{\prime}$ and

$$
\operatorname{Dim}\left(\mathbb{E}\left(\mathbb{A}^{(s)} \mid \mathbb{X}^{\prime}\right)\right) \leqslant \operatorname{Dim}\left(\mathbb{E}\left(\mathbb{A}^{\prime} \mid \mathbb{X}^{\prime}\right)\right)=\operatorname{Dim}\left(\gamma \odot \mathbb{X}^{\prime}\right)=\gamma
$$

which yields that the set $\left\{s \in S: \mathbb{E}\left(\mathbb{A}^{(s)} \mid \mathbb{X}^{\prime}\right) \neq \mathbb{O}\right\}$ has power $\gamma^{+}$. Conversely, if $\mathbb{Y}$ is a member of the right-hand side set appearing in (13-3), then necessarily $\mathbb{Y} \perp_{u} \mathbb{E}_{\gamma^{+}}^{i}\left(\mathbb{A}^{\prime}\right)$ and $\mathbb{Y} \leqslant \mathbb{E}_{\gamma}^{i}(\mathbb{A}) \boxplus \mathbb{E}_{\gamma^{+}}^{i}(\mathbb{A})$. So, we only need to show that $\mathbb{Y} \perp_{u} \mathbb{E}_{\gamma}^{i}(\mathbb{A})$. Suppose, for the contrary, that $\mathbb{Y}^{\prime}=\mathbb{Y} \wedge \mathbb{E}_{\gamma}^{i}(\mathbb{A})\left(\leqslant^{s} \mathbb{Y}\right)$ is nontrivial. Then there is $\mathbb{X} \leqslant{ }^{s} \mathbb{Y}^{\prime}$ such that $\operatorname{Dim}(\mathbb{X})=\aleph_{0}$. Observe that $\bigoplus_{s \in S} \mathbb{E}\left(\mathbb{A}^{(s)} \mid \mathbb{X}\right)=\mathbb{E}(\mathbb{A} \mid \mathbb{X})=\gamma \odot \mathbb{X}$ which denies the fact that $\operatorname{card}\left(\left\{s \in S: \mathbb{E}\left(\mathbb{A}^{(s)} \mid \mathbb{X}\right) \neq \mathbb{O}\right\}\right)=\gamma^{+}$.

One may deduce from (13-2) and (13-3) that (below we use the notation introduced in this section)

$$
\mathbb{A}=\mathbb{A}^{\prime} \vee\left[\gamma^{+} \odot\left(\mathbb{E}_{\gamma^{+}}(\mathbb{A}) \boxminus \mathbb{E}_{\gamma^{+}}\left(\mathbb{A}^{\prime}\right)\right)\right]
$$

The above notices show that Example 8.9 (page 21) demonstrates all reasons for which it may happen that (12-1) is false. We end the section with the announced

13.2. Theorem. Let $S$ be a nonempty set and $\left\{\mathcal{A}_{s}\right\}_{s \in S}$ be a collection of nonempty subsets of $\mathrm{CDD}_{N}$. Then

$$
\bigvee\left(\bigoplus_{s \in S} \mathcal{A}_{s}\right)=\bigoplus_{s \in S}\left(\bigvee \mathcal{A}_{s}\right)
$$

where $\bigoplus_{s \in S} \mathcal{A}_{s}=\left\{\bigoplus_{s \in S} \mathbb{X}^{(s)}: \mathbb{X}^{(s)} \in \mathcal{A}_{s}(s \in S)\right\}$.

Proof. The inequality ' $\leqslant$ ' in (13-5) is clear. We shall prove the converse one by transfinite induction on $\operatorname{card}(S)$. The cases when $\operatorname{card}(S)<\aleph_{0}$ or card $(S)=\aleph_{0}$ are included in Proposition 12.2 (page 31) and Corollary 12.3 (page 32), respectively. Assume $\beta$ is an uncountable cardinal such that $(13-5)$ is fulfilled $\operatorname{provided} \operatorname{card}(S)<\beta$. Now suppose $\operatorname{card}(S)=\beta$. If $\beta$ is limit, the assertion (i.e. the inequality ' $\geqslant$ ' in (13-5)) follows from (AO6) (page 29) and the transfinite induction hypothesis. Thus we may assume that $\beta=\gamma^{+}$. Put $\mathcal{A}=\bigoplus_{s \in S} \mathcal{A}_{s}$, $\mathbb{A}^{(s)}=\bigvee \mathcal{A}_{s}(s \in S), \mathbb{A}=\bigoplus_{s \in S} \mathbb{A}^{(s)}$ and $\mathbb{A}^{\prime}=\bigvee\left\{\bigoplus_{s \in S^{\prime}} \mathbb{A}^{(s)}: S^{\prime} \subset\right.$ $\left.S, 0<\operatorname{card}\left(S^{\prime}\right) \leqslant \gamma\right\}$. We infer from the transfinite induction hypothesis that $\mathbb{A}^{\prime} \leqslant \bigvee \mathcal{A}$. Hence, according to (13-4), we only need to show 
that $\gamma^{+} \odot\left(\mathbb{E}_{\gamma^{+}}(\mathbb{A}) \boxminus \mathbb{E}_{\gamma^{+}}\left(\mathbb{A}^{\prime}\right)\right) \leqslant \bigvee \mathcal{A}$. Having in mind the partition of unity induced by $\bigvee \mathcal{A}$, we see that the latter inequality will be fulfilled if only

$$
\mathbb{E}_{\gamma^{+}}(\mathbb{A}) \boxminus \mathbb{E}_{\gamma^{+}}\left(\mathbb{A}^{\prime}\right) \perp_{u} \mathbb{E}_{\alpha}^{i}(\bigvee \mathcal{A})
$$

for every $(i, \alpha) \in \Upsilon$ with $\alpha \leqslant \gamma$. Suppose (13-6) is false for some $\alpha \leqslant \gamma$. Then Proposition 9.10 (page 24) implies that there is $\mathbb{X} \in \mathcal{C D} \mathcal{D}_{N}$ such that $0<\operatorname{dim}(\mathbb{X}) \leqslant \aleph_{0}$,

$$
\mathbb{X} \leqslant \mathbb{E}_{\alpha}^{i}(\bigvee \mathcal{A}) \quad \text { and } \quad \mathbb{X} \leqslant \leqslant^{s} \mathbb{E}_{\gamma^{+}}(\mathbb{A}) \boxminus \mathbb{E}_{\gamma^{+}}\left(\mathbb{A}^{\prime}\right)
$$

The first relation of $(13-7)$ yields that $\mathbb{E}(\bigvee \mathcal{A} \mid \mathbb{X}) \leqslant \alpha \odot \mathbb{X}$ (by the characterization of $\mathbb{E}_{\alpha}^{i}(\bigvee \mathcal{A})$ given in Theorem 11.1, page 26). Consequently,

$$
\operatorname{dim}\left(\mathbb{E}\left(\bigoplus_{s \in S} \mathbb{B}^{(s)} \mid \mathbb{X}\right)\right) \leqslant \alpha
$$

whenever $\mathbb{B}^{(s)} \in \mathcal{A}_{s}(s \in S)$. But $\mathbb{E}\left(\bigoplus_{s \in S} \mathbb{B}^{(s)} \mid \mathbb{X}\right)=\bigoplus_{s \in S} \mathbb{E}\left(\mathbb{B}^{(s)} \mid \mathbb{X}\right)$ and thus (13-8) changes into $\sum_{s \in S} \operatorname{dim}\left(\mathbb{E}\left(\mathbb{B}^{(s)} \mid \mathbb{X}\right)\right) \leqslant \alpha$. So, we have obtained that whatever $\mathbb{B}^{(s)} \in \mathcal{A}_{s}$ we choose,

$$
\operatorname{card}\left(\left\{s \in S: \mathbb{B}^{(s)} \not_{u} \mathbb{X}\right\}\right) \leqslant \gamma
$$

However, the second relation of (13-7) combined with (13-3) yields that the set $S^{\prime}=\left\{s \in S: \mathbb{A}^{(s)} \not_{u} \mathbb{X}\right\}$ has power $\gamma^{+}$. Observe that for $s \in S$, if $\mathbb{Y} \perp_{u} \mathbb{X}$ for every $\mathbb{Y} \in \mathcal{A}_{s}$, then necessarily $\mathbb{A}^{(s)}=\bigvee \mathcal{A}_{s} \perp_{u} \mathbb{X}$ and hence $s \notin S^{\prime}$. We conclude from this that for every $s \in S^{\prime}$ there is $\mathbb{B}^{(s)} \in \mathcal{A}_{s}$ such that $\mathbb{B}^{(s)} \not_{u} \mathbb{X}$. Now (13-9) denies the fact that $\operatorname{card}\left(S^{\prime}\right)=\gamma^{+}$. Consequently, (13-6) is satisfied and we are done.

\section{Semigroup of Semiminimal $N$-tuples}

This section is devoted to a deeper study of $\mathcal{S M}_{N}$. Thanks to (AO4) (page 29$), \mathcal{S M}_{N}$ is a set and $\left(\mathcal{S M N}_{N}, \oplus\right)$ is a semigroup which may be enlarged to an Abelian group.

A similar construction to the following may be found in [9] (see Proposition 1.41 there). Fix nontrivial $\mathbb{A} \in \mathcal{S} \mathcal{M}_{N}$. Since $\mathcal{W}^{\prime}(\boldsymbol{A})$ is type $\mathrm{II}_{1}$, for every $n \geqslant 1$ there is a unique (by (AO1), page 29) $\mathbb{A}^{(n)} \in \mathcal{S \mathcal { M }}_{N}$ such that $\mathbb{A}=n \odot \mathbb{A}^{(n)}$. Notation: $\frac{1}{n} \odot \mathbb{A}=\mathbb{A}^{(n)}$. Now if $w$ is a positive rational number and $w=\frac{p}{q}$ with natural coprime $p$ and $q$, we define $w \odot \mathbb{A}=p \odot\left(\frac{1}{q} \odot \mathbb{A}\right)$. Finally, for a positive real number $t$ let

$$
t \odot \mathbb{A}=\bigvee\{w \odot \mathbb{A}: w \in \mathbb{Q}, w \leqslant t\} .
$$

Additionally, put $t \odot \mathbb{O}=\mathbb{O}$ for each $t \in \mathbb{R}_{+}$. Using traces on *commutants of semiminimal $N$-tuples (i.e. on $\mathcal{W}^{\prime}(A)$ for $\mathbb{A} \in \mathcal{S} \mathcal{M}_{N}$ ), one shows that for every $s, t \in \mathbb{R}_{+}$and any $\mathbb{A}, \mathbb{B} \in \mathcal{S M}_{N}$, $(\mathrm{VS} 1) 0 \odot \mathbb{A}=\mathbb{O} ; 1 \odot \mathbb{A}=\mathbb{A}$, 
$(\mathrm{VS} 2) s \odot \mathbb{A} \leqslant t \odot \mathbb{A}$ provided $s \leqslant t$,

$(\mathrm{VS} 3) t \odot \mathbb{A}=\bigwedge\{x \odot \mathbb{A}: x>t\}$ and for $t>0, t \odot \mathbb{A}=\bigvee\{x \odot \mathbb{A}: 0 \leqslant$ $x<t\}$

$(\mathrm{VS} 4)(s t) \odot \mathbb{A}=s \odot(t \odot \mathbb{A}) ;(s+t) \odot \mathbb{A}=(s \odot \mathbb{A}) \oplus(t \odot \mathbb{A})$,

$(\mathrm{VS} 5) t \odot(\mathbb{A} \oplus \mathbb{B})=(t \odot \mathbb{A}) \oplus(t \odot \mathbb{B})$

(VS6) if ' $\sim$ ' denotes one of ' $\leqslant$ ', ' $\leqslant$ ', ' $\ll$ ', ' $\perp_{u}$ ' and $t>0$, then $t \odot \mathbb{A} \sim$ $t \odot \mathbb{B} \Longleftrightarrow \mathbb{A} \sim \mathbb{B}$,

(VS7) if $\mathbb{A}=\bigoplus_{s \in S} \mathbb{A}^{(s)}$, then $t \odot \mathbb{A}=\bigoplus_{s \in S}\left(t \odot \mathbb{A}^{(s)}\right.$ ) (this follows from (VS5), (VS6) and Proposition 12.6, page 33),

$(\mathrm{VS} 8) \mathfrak{b}(\mathbb{A}) \in \mathcal{S M}_{N}$ and $\mathfrak{b}(t \odot \mathbb{A})=t \odot \mathfrak{b}(\mathbb{A})$

(VS9) for every sequence $\left(t_{n}\right)_{n=1}^{\infty}$ of nonnegative reals, $\left(\sum_{n=1}^{\infty} t_{n}\right) \odot \mathbb{A}=$ $\bigoplus_{n=1}^{\infty} t_{n} \odot \mathbb{A}$ (where $\infty$ is identified with $\aleph_{0}$, if needed).

Now by (VS1), (VS4), (VS5) and (AO4) (page 29), there is a real vector space

$$
\left(\mathcal{E}_{N},+, \cdot\right) \supset\left(\mathcal{S M K}_{N}, \oplus, \odot\right) .
$$

The above inclusion means that the addition and multiplication by reals in $\mathcal{E}_{N}$ extend ' $\oplus$ ' and the just defined ' $\odot$ '. $\mathcal{S} \mathcal{M}_{N}$ as a subset of $\mathcal{E}_{N}$ is a cone $\left(\mathcal{S} \mathcal{M}_{N}+\mathcal{S} \mathcal{M}_{N} \subset \mathcal{S} \mathcal{M}_{N}, \mathbb{R}_{+} \cdot \mathcal{S} \mathcal{M}_{N} \subset \mathcal{S} \mathcal{M}_{N}\right.$ and $\mathcal{S} \mathcal{M}_{N} \cap\left(-\mathcal{S} \mathcal{M}_{N}\right)=$ $\{0\}=\{\mathbb{O}\}$ ). We may assume that $\mathcal{E}_{N}=\mathcal{S} \mathcal{M}_{N}-\mathcal{S} \mathcal{M}_{N}$. Under such an assumption, we may consider the partial order on $\mathcal{E}_{N}$ induced by $\mathcal{S M}_{N}: \xi_{1} \leqslant \varepsilon \xi_{2} \Longleftrightarrow \xi_{2}-\xi_{1} \in \mathcal{S M}_{N}\left(\xi_{1}, \xi_{2} \in \mathcal{E}_{N}\right)$. It may be checked that for $\mathbb{A}, \mathbb{B} \in \mathcal{S \mathcal { M }}_{N}, \mathbb{A} \leqslant \varepsilon \mathbb{B} \Longleftrightarrow \mathbb{A} \leqslant \mathbb{B}$. So, ' $\leqslant \varepsilon$ ' extends ' $\leqslant$ ' and therefore we shall omit the subscript ' $\mathcal{E}$ ' in ' $\leqslant \mathcal{E}$ ' and we shall simply write ' $\leqslant$ ' instead of ' $\leqslant \varepsilon$ '. Since every nonempty subset of $\mathcal{S} \mathcal{M}_{N}$ which is upper bounded in $\mathcal{S \mathcal { N }}_{N}$ has the l.u.b. $\left(\operatorname{in} \mathcal{S M}_{N}\right), \mathcal{E}_{N}$ is a conditionally complete lattice (which means that every nonempty upper bounded subset of $\mathcal{E}_{N}$ has the l.u.b. in $\mathcal{E}_{N}$ ). Our aim is to find a 'model' for the lattice $\varepsilon_{N}$.

From now to the end of the section we fix a representative $\boldsymbol{J}_{I I}$ of $\mathbb{J}_{I I}$, a compact Hausdorff space $\Omega_{I I}$ homeomorphic to the Gelfand spectrum of $\mathcal{Z}\left(\mathcal{W}^{\prime}\left(\boldsymbol{J}_{I I}\right)\right)$ and an isomorphism $\Psi: \mathcal{Z}\left(\mathcal{W}^{\prime}\left(\boldsymbol{J}_{I I}\right)\right) \rightarrow \mathcal{C}\left(\Omega_{I I}\right)$ of $*$-algebras where $\mathcal{C}\left(\Omega_{I I}\right)$ is the algebra of all continuous complex-valued functions on $\Omega_{I I}$. Every $\mathbb{A} \leqslant^{s} \mathbb{J}_{I I}$ corresponds to a unique central projection $z_{\mathbb{A}}$ in $\mathcal{W}^{\prime}\left(\boldsymbol{J}_{I I}\right)$. Let $U_{\mathbb{A}}$ be a clopen (i.e. simultaneously closed and open) subset of $\Omega_{I I}$ whose characteristic function coincides with $\Psi\left(z_{\mathbb{A}}\right)$. $\Omega_{I I}$ is extremely disconnected (that is, the closure of every open subset of $\Omega_{I I}$ is open as well; see [18, Theorem 5.2.1]) and the assignment $\mathbb{A} \mapsto U_{\mathbb{A}}$ establishes a one-to-one correspondence between all $N$-tuples $\mathbb{X} \in \mathcal{S \mathcal { N }}_{N}^{\infty}\left(\right.$ where $\mathcal{S \mathcal { M }}_{N}^{\infty}=\left\{\mathbb{X} \in \mathcal{C D D}_{N}: \mathbb{X} \leqslant \leqslant^{s} \mathbb{J}_{I I}\right\}=\left\{\aleph_{0} \odot \mathbb{A}: \mathbb{A} \in\right.$ $\left.\left.\mathcal{S \mathcal { M }}_{N}\right\}\right)$ and all clopen subsets of $\Omega_{I I}$. Moreover, for $\mathbb{A}, \mathbb{B} \in \mathcal{S \mathcal { M }}_{N}^{\infty}$, $\mathbb{A} \leqslant^{s} \mathbb{B} \Longleftrightarrow U_{\mathbb{A}} \subset U_{\mathbb{B}}$.

For every $\mathbb{A} \in \mathcal{S} \mathcal{M}_{N}, \widetilde{\mathbb{A}}=\aleph_{0} \odot \mathbb{A} \leqslant^{s} \mathbb{J}_{I I}$ and thus $U_{\widetilde{\mathbb{A}}}$ makes sense. The latter set is said to be the support of $\mathbb{A}$ and denoted by $\operatorname{supp}_{\Omega_{I I}} \mathbb{A}$. There is no difficulty in verifying that $\operatorname{supp}_{\Omega_{I I}} \mathbb{A} \subset \operatorname{supp}_{\Omega_{I I}} \mathbb{B}$ 
(respectively supp $\Omega_{\Omega_{I I}} \mathbb{A} \cap \operatorname{supp}_{\Omega_{I I}} \mathbb{B}=\varnothing$ ) provided $\mathbb{A} \ll \mathbb{B}$ (respectively $\left.\mathbb{A} \perp_{u} \mathbb{B}\right)$ and $\mathbb{A}, \mathbb{B} \in \mathcal{S} \mathcal{M}_{N}$.

The following idea comes from theory of $\mathcal{W}^{*}$-algebras (compare with [18, Definition 5.6.5]) especially when working with the so-called extended center valued traces (see the notes on page 329 of [34] and Definition V.2.33 there). We consider the set

$$
\mathcal{M}\left(\Omega_{I I}\right)=\left\{f \in \mathcal{C}\left(\Omega_{I I},[-\infty,+\infty]\right): f^{-1}(\mathbb{R}) \text { is dense in } \Omega_{I I}\right\} .
$$

To make the space $\mathcal{M}\left(\Omega_{I I}\right)$ a real vector space, we need the following well-known result (it follows from [18, Corollary 5.2.11] or [34, Corollary III.1.8]; see also [12] for more general results in this direction).

14.1. Lemma. If $X$ and $K$ are compact Hausdorff spaces and $X$ is extremely disconnected, then every continuous function of an arbitrary open dense subset of $\mathcal{X}$ into $K$ is extendable to a continuous function of $X$ into $K$.

Now if $f, g \in \mathcal{M}\left(\Omega_{I I}\right)$, the set $D=f^{-1}(\mathbb{R}) \cap g^{-1}(\mathbb{R})$ is open and dense in $\Omega_{I I}$ and the function $\left.f\right|_{D}+\left.g\right|_{D}$ is well defined and continuous. Consequently, thanks to Lemma 14.1, there is a unique member of $\mathcal{M}\left(\Omega_{I I}\right)$, which we shall denote by $f+g$, which coincides with the usual sum on $D$. Similarly one defines $f \cdot g$ and $t \cdot f$ for $t \in \mathbb{R}$. We leave this as an exercise that $\mathcal{M}\left(\Omega_{I I}\right)$ is a real vector space with these operations. Further, we equip $\mathcal{N}\left(\Omega_{I I}\right)$ with the pointwise order. One may easily check that $\mathcal{M}\left(\Omega_{I I}\right)$ is a lattice (i.e. every finite nonempty subset of $\mathcal{M}\left(\Omega_{I I}\right)$ has the l.u.b. and g.l.b.). What is more, $\mathcal{M}\left(\Omega_{I I}\right)$ is conditionally complete, since $\Omega_{I I}$ is extremely disconnected (it follows from [34, Proposition III.1.7]). In the sequel we shall show that $\mathcal{E}_{N}$ and $\mathcal{M}\left(\Omega_{I I}\right)$ are lattice-isomorphic. For every $f \in \mathcal{M}\left(\Omega_{I I}\right)$ let supp $f$ be the closure of the set $\left\{x \in \Omega_{I I}: f(x) \neq 0\right\}$. Since $\Omega_{I I}$ is extremely disconnected, $\operatorname{supp} f$ is clopen.

When $\mathcal{X}$ is a clopen subset of $\Omega_{I I}$, let $\mathcal{N}\left(\Omega_{I I} \mid \mathcal{X}\right)$ be the set of all $f \in$ $\mathcal{M}\left(\Omega_{I I}\right)$ for which supp $f \subset \mathcal{X} \cdot \mathcal{M}\left(\Omega_{I I} \mid \mathcal{X}\right)$ is a sublattice of $\mathcal{M}\left(\Omega_{I I}\right)$. By $\mathcal{M}_{+}\left(\Omega_{I I}\right)$ and $\mathcal{M}_{+}\left(\Omega_{I I} \mid \mathcal{X}\right)$ we denote the cones of nonnegative elements of the suitable lattices.

For the next step of our considerations we need

14.2. Lemma. Let $\Omega$ be the Gelfand spectrum of a commutative $\mathcal{W}^{*}$ algebra. Every dense $\mathcal{G}_{\delta}$ subset of $\Omega$ has dense interior. What is more, for each Borel function $f: \Omega \rightarrow \mathbb{R}$ there is an open dense set $D \subset \Omega$ such that $\left.f\right|_{D}$ is continuous.

Lemma 14.2 follows from [18, Lemma 5.2.10] combined with Proposition III.1.15 and Theorem III.1.17 of [34] (see also the statement preceding Corollary III.1.16 there).

Let $\left\{f_{n}\right\}_{n=1}^{\infty} \subset \mathcal{M}_{+}\left(\Omega_{I I}\right)$ be such that $\sum_{k=1}^{n} f_{k} \leqslant g$ for some $g \in$ $\mathcal{M}_{+}\left(\Omega_{I I}\right)$ and each $n$. We define $\sum_{n=1}^{\infty} f_{n} \in \mathcal{M}_{+}\left(\Omega_{I I}\right)$ as follows. Let 
$f: \Omega \rightarrow \mathbb{R}$ be given by $f(x)=\sum_{n=1}^{\infty} f_{n}(x)$ provided the latter series is convergent and $f(x)=0$ otherwise. By Lemma 14.2, there is an open dense subset $D$ of $\Omega_{I I}$ such that $\left.f\right|_{D}$ is continuous. We define $\sum_{n=1}^{\infty} f_{n} \in \mathcal{M}_{+}\left(\Omega_{I I}\right)$ as the unique continuous extension of $\left.f\right|_{D}$. Since $f \leqslant g,\left(\sum_{n=1}^{\infty} f_{n}\right)(x)=\sum_{n=1}^{\infty} f_{n}(x)$ for $x$ belonging to an open dense subset of $\Omega_{I I}$. One may check that $\sum_{n=1}^{\infty} f_{n}=\sup \mathcal{M}\left(\Omega_{I I}\right)\left\{\sum_{k=1}^{n} f_{k}\right\}_{n \geqslant 1}$.

Fix nontrivial $\mathbb{X} \in \mathcal{S M}_{N}$. Let $\mathcal{L}[\mathbb{X}]=\left\{\mathbb{F} \in \mathcal{S M}_{N}: \mathbb{F} \ll \mathbb{X}\right\}$ and $\mathcal{X}=\operatorname{supp}_{\Omega_{I I}} \mathbb{X}$. Then we have

14.3. Theorem. There is a unique operator

$$
\mathcal{L}[\mathbb{X}] \ni \mathbb{F} \mapsto \frac{\mathrm{d} \mathbb{F}}{\mathrm{d} \mathbb{X}} \in \mathcal{M}_{+}\left(\Omega_{I I} \mid X\right)
$$

such that for any $\mathbb{F}, \mathbb{F}^{(n)} \in \mathcal{S \mathcal { M }}_{N}(n=1,2,3, \ldots)$ :

(TR0) $\frac{d \mathbb{X}}{d \mathbb{X}}=$ the characteristic function of $X$,

(TR1) $\operatorname{supp} \frac{d \mathbb{F}}{d \mathbb{X}} \subset \operatorname{supp}_{\Omega_{I I}} \mathbb{F}$,

$(\mathrm{TR} 2) \frac{\mathrm{d}\left(\oplus_{n=1}^{\infty} \mathbb{F}^{(n)}\right)}{\mathrm{d} \mathbb{X}}=\sum_{n=1}^{\infty} \frac{\mathrm{d} \mathbb{F}^{(n)}}{\mathrm{d} \mathbb{X}}$ if $\bigoplus_{n=1}^{\infty} \mathbb{F}^{(n)} \in \mathcal{S} \mathcal{M} \mathcal{M}_{N}$ (see the note preceding the theorem).

Moreover, the above operator has further properties:

(TR1') supp $\frac{\mathrm{d} \mathbb{F}}{\mathrm{dX}}=\operatorname{supp}_{\Omega_{I I}} \mathbb{F}$ for every $\mathbb{F} \in \mathcal{L}[\mathbb{X}]$,

(TR2') whenever $\mathbb{A} \in \mathcal{L}[\mathbb{X}]$ is of the form $\mathbb{A}=\bigoplus_{s \in S} \mathbb{A}^{(s)}$,

$$
\frac{\mathrm{d} \mathbb{A}}{\mathrm{d} \mathbb{X}}=\sum_{s \in S} \frac{\mathrm{d} \mathbb{A}^{(s)}}{\mathrm{d} \mathbb{X}}:=\sup \mathcal{M}\left(\Omega_{I I} \mid X\right)\left\{\sum_{s \in S_{0}} \frac{\mathrm{d}^{(s)}}{\mathrm{d} \mathbb{X}}: S_{0} \in \mathcal{P}_{f}(S)\right\},
$$

(TR4) $\frac{\mathrm{d}(t \odot \mathbb{F})}{\mathrm{d} \mathbb{X}}=t \frac{\mathrm{d} \mathbb{F}}{\mathrm{d} \mathbb{X}}$ for each $\mathbb{F} \in \mathcal{L}[\mathbb{X}]$,

(TR5) for any $\mathbb{A}, \mathbb{B} \in \mathcal{L}[\mathbb{X}], \mathbb{A} \leqslant \mathbb{B} \Longleftrightarrow \frac{\mathrm{d} \mathbb{A}}{\mathrm{d} \mathbb{X}} \leqslant \frac{\mathrm{d} \mathbb{B}}{\mathrm{d} \mathbb{X}}$,

(TR6) for every $f \in \mathcal{M}_{+}\left(\Omega_{I I} \mid \mathcal{X}\right)$ there is unique $\mathbb{F} \in \mathcal{L}[\mathbb{X}]$ with $\frac{\mathrm{d} \mathbb{F}}{\mathrm{d} \mathbb{X}}=f$.

Proof. The existence of the operator may be deduced from the result on faithful normal extended center valued traces for semifinite $\mathcal{W}^{*}$ algebras $\left(\left[34\right.\right.$, Theorem V.2.34]) applied to $\mathcal{W}^{\prime}(\boldsymbol{Y})$ with $\mathbb{Y}=\aleph_{0} \odot \mathbb{X}$. The operator may also be constructed as follows. By Theorem 9.6 (page 23), there is $\mathbb{X}^{\prime} \in \mathcal{S} \mathcal{M}_{N}$ such that $\mathbb{J}_{I I}=\aleph_{0} \odot\left(\mathbb{X} \boxplus \mathbb{X}^{\prime}\right)$. Put $\widetilde{\mathbb{X}}=\mathbb{X} \boxplus \mathbb{X}^{\prime}$ and let $\mathcal{M}=\mathcal{W}^{\prime \prime}(\widetilde{\boldsymbol{X}})$. Then $\mathcal{M}^{\prime}=\mathcal{W}^{\prime}(\widetilde{\boldsymbol{X}})$. Since $\widetilde{\mathbb{X}} \in \mathcal{S} \mathcal{M}_{N}$, $\mathcal{M}^{\prime}$ is type $\mathrm{II}_{1}$ and hence there is a trace $\operatorname{tr}: \mathcal{M}^{\prime} \rightarrow \mathcal{Z}\left(\mathcal{M}^{\prime}\right)$ (with $\operatorname{tr}(1)=1)$. Since $\mathcal{Z}\left(\mathcal{M}^{\prime}\right)=\mathcal{Z}(\mathcal{M}), \mathcal{Z}\left(\mathcal{W}^{\prime \prime}\left(\boldsymbol{J}_{I I}\right)\right)=\mathcal{Z}\left(\mathcal{W}^{\prime}\left(\boldsymbol{J}_{I I}\right)\right)$ and a function $\mathcal{M} \ni T \mapsto \aleph_{0} \odot T \in \mathcal{W}^{\prime \prime}\left(\boldsymbol{J}_{I I}\right)$ is an isomorphism of $*$-algebras, a function $\kappa: \mathcal{Z}\left(\mathcal{M}^{\prime}\right) \ni T \mapsto \aleph_{0} \odot T \in \mathcal{Z}\left(\mathcal{W}^{\prime}\left(J_{I I}\right)\right)$ is a well defined *-isomorphism. Define $\operatorname{Tr}: \mathcal{M}^{\prime} \rightarrow \mathcal{C}\left(\Omega_{I I}\right)$ by $\operatorname{Tr}=\Psi \circ \kappa \circ$ tr. Now if $\mathbb{A} \ll \mathbb{X}$, by Definition 9.5 (page 23), $\mathbb{A}$ may be written in the form $\mathbb{A}=\boxplus_{n=1}^{\infty} \mathbb{A}^{(n)}$ with $\mathbb{A}^{(n)} \leqslant n \odot \mathbb{X}$. The latter implies that $\frac{1}{n} \odot \mathbb{A}^{(n)} \leqslant \widetilde{\mathbb{X}}$ and thus there is a projection $p_{n}$ in $\mathcal{M}^{\prime}(\widetilde{\boldsymbol{X}})$ which corresponds (by 
Proposition 4.1 , page 9$)$ to $\frac{1}{n} \odot \mathbb{A}^{(n)}$. We put

$$
\frac{\mathrm{d} \mathbb{A}}{\mathrm{d} \mathbb{X}}=\sum_{n=1}^{\infty} n \operatorname{Tr}\left(p_{n}\right) \text {. }
$$

Since $\mathbb{A}^{(n)} \perp_{u} \mathbb{A}^{(m)}$ for $n \neq m$, supp $\operatorname{Tr}\left(p_{n}\right) \cap \operatorname{supp} \operatorname{Tr}\left(p_{m}\right)=\varnothing$ and thus (14-1) is well understood, by Lemma 14.1. We leave this as an exercise that such a definition is independent of the choice of $\left(\mathbb{A}^{(n)}\right)_{n=1}^{\infty}$ and that all conditions of the theorem are fulfilled (observe that $\mathbb{X}$ corresponds to a central projection in $\left.\mathcal{M}^{\prime}(\tilde{X})\right)$. Here we focus on the uniqueness of the operator.

When $\mathbb{A} \leqslant^{s} \mathbb{X}$, then $\mathbb{X}=\mathbb{A} \boxplus \mathbb{B}$ with $\mathbb{B}=\mathbb{X} \boxminus \mathbb{A}$ and $\operatorname{supp}_{\Omega_{I I}} \mathbb{B}=$ $\operatorname{supp}_{\Omega_{I I}} \mathbb{X} \backslash \operatorname{supp}_{\Omega_{I I}} \mathbb{A}$. Consequently, by (TR0)-(TR2), $\frac{\mathrm{d} \mathbb{A}}{\mathrm{d} \mathbb{X}}$ is the characteristic function of $\operatorname{supp}_{\Omega_{I I}} \mathbb{A}$. This shows that $\frac{d \mathbb{F}}{d \mathbb{X}}$ is uniquely determined by $(\mathrm{TR} 0)-(\mathrm{TR} 2)$ for $\mathbb{F} \in \mathcal{F}_{0}:=\left\{w \odot \mathbb{A}: w \in \mathbb{Q}_{+}, \mathbb{A} \leqslant^{s} \mathbb{X}\right\}$.

Further, if $\mathbb{A} \leqslant \mathbb{X}$, then $\mathbb{A}$ may be written in the form $\mathbb{A}=\bigoplus_{n=1}^{\infty} \mathbb{F}^{(n)}$ with $\mathbb{F}^{(n)} \in \mathcal{F}_{0}$ (this may be deduced, by means of the trace, from the representation of a continuous function on an extremely disconnected compact Hausdorff space as a series of continuous functions with finite ranges). So, according to (TR2), $\frac{d \mathbb{B}}{d \mathbb{X}}$ is uniquely determined by (TR0)(TR2) for $\mathbb{B}=w \odot \mathbb{A}$ with rational $w$ and $\mathbb{A} \leqslant \mathbb{X}$. To this end, it suffices to recall that if $\mathbb{A} \in \mathcal{L}[\mathbb{X}]$, then $\mathbb{A}=\boxplus_{n=1}^{\infty} \mathbb{A}^{(n)}$ with $\frac{1}{n} \odot \mathbb{A}^{(n)} \leqslant \mathbb{X}$.

14.4. Corollary. $\mathcal{E}_{N}$ and $\mathcal{M}\left(\Omega_{I I}\right)$ are isomorphic as ordered vector spaces.

Proof. Take $\mathbb{X} \in \mathcal{S} \mathcal{M}_{N}$ such that $\aleph_{0} \odot \mathbb{X}=\mathbb{J}_{I I}$ and define $\Phi_{+}: \mathcal{S M}_{N} \ni$ $\mathbb{F} \mapsto \frac{\mathrm{d} \mathbb{F}}{\mathrm{d}} \in \mathcal{M}_{+}\left(\Omega_{I I}\right)$. By Theorem 14.3, $\Phi_{+}$is an additive bijection preserving orders. Now it suffices to extend $\Phi_{+}$in a standard way: $\Phi(\xi)=\Phi\left(\xi_{+}\right)-\Phi\left(\xi_{-}\right)$.

14.5. Proposition. If $\mathbb{A}, \mathbb{X}, \mathbb{Y} \in \mathcal{S M}_{N}$ are such that $\mathbb{A} \ll \mathbb{X} \ll \mathbb{Y}$, then

$$
\frac{d \mathbb{A}}{d \mathbb{Y}}=\frac{d \mathbb{A}}{d \mathbb{X}} \cdot \frac{d \mathbb{X}}{d \mathbb{Y}}
$$

Proof. Arguing as in the uniqueness part of Theorem 14.3, we only need to check that (14-2) is fulfilled for $\mathbb{A} \leqslant s \mathbb{X}$. When $\mathbb{A}=\mathbb{X},(14-2)$ is clear. So, for arbitrary $\mathbb{A} \leqslant^{s} \mathbb{X},(14-2)$ follows from (TR1) and (TR2).

We end the section with the following two remarks.

14.6. Remark. The notation ' $\frac{\mathrm{d} \mathbb{A}}{\mathrm{d}}$ ' suggests to denote the inverse operator, of $\mathcal{M}_{+}\left(\Omega_{I I} \mid X\right)$ onto $\mathcal{L}[\mathbb{X}]$, by $\int f \mathrm{~d} \mathbb{X}$. Thus, for $f \in \mathcal{M}_{+}\left(\Omega_{I I} \mid X\right)$, $\int f \mathrm{~d} \mathbb{X}=\mathbb{B}$ iff $\mathbb{B} \in \mathcal{L}[\mathbb{X}]$ is such that $\frac{d \mathbb{B}}{d \mathbb{X}}=f$. Arguing similarly as in the proof of Theorem 14.3, one may show that the operator $\mathcal{M}_{+}\left(\Omega_{I I} \mid X\right) \ni f \mapsto \int f d \mathbb{X} \in \mathcal{L}[\mathbb{X}]$ is uniquely determined by the following three conditions: 
(AD1) $\int j_{x} \mathrm{~d} \mathbb{X}=\mathbb{X}$ where $j_{x}$ is the characteristic function of $X$,

$(\mathrm{AD} 2) \operatorname{supp}_{\Omega_{I I}}\left(\int f \mathrm{~d} \mathbb{X}\right) \subset \operatorname{supp} f$ for each $f \in \mathcal{M}_{+}\left(\Omega_{I I} \mid X\right)$,

(AD3) whenever $f \in \mathcal{M}_{+}\left(\Omega_{I I} \mid \mathcal{X}\right)$ has the form $f=\sum_{n=1}^{\infty} f_{n}$ (with $\left.f_{n} \in \mathcal{M}_{+}\left(\Omega_{I I} \mid X\right)\right)$,

$$
\int f \mathrm{~d} \mathbb{X}=\bigoplus_{n=1}^{\infty} \int f_{n} \mathrm{~d} \mathbb{X}
$$

It seems to be interesting that (AD3) reminds classical Lebesgue's monotone convergence theorem.

14.7. Remark. Specialists in Hilbert space operators probably would prefer the version of ' $\frac{d \mathbb{Y}}{d \mathbb{X}}$ ' whose values are operators rather than functions. This is possible and may be provide as follows. Since every bounded member of $\mathcal{M}_{+}\left(\Omega_{I I}\right)$ corresponds, by $\Psi$, to a nonnegative element of $\mathcal{Z}\left(\mathcal{W}^{\prime}\left(\boldsymbol{J}_{I I}\right)\right)$, each member of $\mathcal{M}_{+}\left(\Omega_{I I}\right)$ corresponds to a (possibly unbounded) nonnegative selfadjoint operator $A$ such that $\mathfrak{b}(A) \in \mathcal{Z}\left(\mathcal{W}^{\prime}\left(\boldsymbol{J}_{I I}\right)\right)$ (in von Neumann algebra theory such an operator $A$ is said to be affiliated with $\mathcal{Z}\left(\mathcal{W}^{\prime}\left(\boldsymbol{J}_{I I}\right)\right)$; see e.g. [18, Definition 5.6.2]). Thus, if we let $\boldsymbol{L}[\boldsymbol{X}]$ and $\widehat{\mathcal{Z}}_{+}\left(\mathcal{W}^{\prime}\left(\boldsymbol{J}_{I I}\right)\right)$ denote, respectively, the classes of all $Y \in \mathrm{CDD}_{N}$ whose unitary equivalence class is semiminimal and which are covered by $\boldsymbol{X}$ (i.e. $\boldsymbol{Y} \ll \boldsymbol{X}$ ) and of all last mentioned operators $A$, then Theorem 14.3 may be adapted to these settings in such a way that $\frac{\mathrm{d} \boldsymbol{Y}}{\mathrm{d} \boldsymbol{X}} \in \widehat{\mathcal{Z}}_{+}\left(\mathcal{W}^{\prime}(X)\right)$ for any $\boldsymbol{Y} \in \boldsymbol{L}[\boldsymbol{X}]$ and (here we list only these properties which do not need additional explanations): (a) $\frac{\mathrm{d} \boldsymbol{X}}{\mathrm{d} \boldsymbol{X}}$ is the unit of $\mathcal{Z}\left(\mathcal{W}^{\prime}(\boldsymbol{X})\right)$ (so, $\frac{\mathrm{d} \boldsymbol{X}}{\mathrm{d} \boldsymbol{X}}$ is a central projection in $\mathcal{W}^{\prime}\left(\boldsymbol{J}_{I I}\right)$ ); (b) $\frac{\mathrm{d} \boldsymbol{Y}^{\prime}}{\mathrm{d} \boldsymbol{X}}=\frac{\mathrm{d} \boldsymbol{Y}^{\prime \prime}}{\mathrm{d} \boldsymbol{X}}$ iff $\boldsymbol{Y}^{\prime}$ and $\boldsymbol{Y}^{\prime \prime}$ are unitarily equivalent; (c) if $\boldsymbol{A} \leqslant m \odot \boldsymbol{X}$ and $\boldsymbol{B} \leqslant n \odot \boldsymbol{X}$ for some natural numbers $m$ and $n$, then both $\frac{\mathrm{d} \boldsymbol{A}}{\mathrm{d} \boldsymbol{X}}$ and $\frac{\mathrm{d} \boldsymbol{B}}{\mathrm{d} \boldsymbol{X}}$ are bounded and $\frac{\mathrm{d}(\boldsymbol{A} \oplus \boldsymbol{B})}{\mathrm{d} \boldsymbol{X}}=\frac{\mathrm{d} \boldsymbol{A}}{\mathrm{d} \boldsymbol{X}}+\frac{\mathrm{d} \boldsymbol{B}}{\mathrm{d} \boldsymbol{X}}$; (d) if $\boldsymbol{Y}_{t}$ is such that $\mathbb{Y}_{t}=t \odot \mathbb{Y}$ (for some $\boldsymbol{Y} \in \boldsymbol{L}[\boldsymbol{X}]$ and $t>0$ ), then $\frac{\mathrm{d} \boldsymbol{Y}_{t}}{\mathrm{~d} \boldsymbol{X}}=t \frac{\mathrm{d} \boldsymbol{Y}}{\mathrm{d} \boldsymbol{X}}$. The reader interested in this approach should consult [18, Theorem 5.6.15].

\section{MODEL FOR $\mathcal{C D} \mathcal{D}_{N}$}

Now we shall develop the idea of the previous part. This will also be an adaptation of the dimension theory for $\mathcal{W}^{*}$-algebras. Let $J$ be a representative of $\mathbb{J}, \Omega$ be a compact Hausdorff space homeomorphic to the Gelfand spectrum of $\mathcal{Z}\left(\mathcal{W}^{\prime}(\boldsymbol{J})\right)$ and let $\Psi: \mathcal{Z}\left(\mathcal{W}^{\prime}(\boldsymbol{J})\right) \rightarrow \mathcal{C}(\Omega)$ be an isomorphism of $*$-algebras. When the triple $(\boldsymbol{J}, \Omega, \Psi)$ is fixed, $\mathbb{J}_{i}$ for $i=I, I I, I I I$ corresponds to a clopen subset $\Omega_{i}$ of $\Omega$. In what follows, we assume that Card $\cap \mathbb{R}_{+}=\mathbb{Z} \cap \mathbb{R}_{+}$. We add and multiply two reals and two infinite cardinals in the usual way and additionally, we put $0 \cdot \alpha=\alpha \cdot 0=0$ and $t+\alpha=\alpha+t=\alpha+0=0+\alpha=\alpha=t \cdot \alpha=\alpha \cdot t$ for $t \in \mathbb{R}_{+} \backslash\{0\}$ and $\alpha \in \operatorname{Card}_{\infty}$. We also extend the natural total orders on $\mathbb{R}_{+}$and $\operatorname{Card}_{\infty}$ assuming that $t<\alpha$ for every real $t$ and an infinite cardinal $\alpha$. In this way the order on $\mathbb{R}_{+} \cup$ Card is total and complete. We equip every set $Y \subset \mathbb{R}_{+} \cup$ Card with the topology inherited from 
the one of the linearly ordered space $I_{\alpha}:=\left\{\xi \in \mathbb{R}_{+} \cup\right.$ Card: $\left.\xi \leqslant \alpha\right\}$ where $\alpha=\sup \left(Y \cup\left\{\aleph_{0}\right\}\right)$ (cf. [8, Problem 1.7.4]). Since the topology of the linearly ordered space $I_{\alpha}$ coincides with the one inherited from the topology of the linearly ordered space $I_{\beta}$ whenever $\aleph_{0} \leqslant \alpha<\beta$, such a definition of the topology on $Y$ makes no confusion. For every topological space $X$, we call a function $f: X \rightarrow \mathbb{R}_{+} \cup$ Card continuous if $f$ is continuous as a function of $X$ into $f(X)$. One may check that for every $\alpha \in \operatorname{Card}_{\infty}, I_{\alpha}$ is compact, the order is a closed subset of $I_{\alpha} \times I_{\alpha}$ and functions $I_{\alpha} \times I_{\alpha} \ni\left(\xi, \xi^{\prime}\right) \mapsto \xi+\xi^{\prime} \in I_{\alpha}$ and $I_{\alpha} \times I_{\alpha} \ni$ $\left(\xi, \xi^{\prime}\right) \mapsto \xi \cdot \xi^{\prime} \in I_{\alpha}$ are continuous.

Let $\Lambda(\Omega)$ be the class of all continuous functions $u: \Omega \rightarrow \mathbb{R}_{+} \cup$ Card such that $u\left(\Omega_{I}\right) \subset$ Card and $u\left(\Omega_{I I I}\right) \subset\{0\} \cup \operatorname{Card}_{\infty}$. We add and multiply members of $\Lambda(\Omega)$ pointwisely. We shall also multiply elements of $\Lambda(\Omega)$ by cardinal numbers pointwisely and we equip $\Lambda(\Omega)$ with the pointwise order. For each $f \in \Lambda(\Omega)$, supp $f$ is the closure of the (open) set $\{x \in \Omega: f(x) \neq 0\}$. Observe that supp $f$ is clopen.

Suppose $\left\{f_{s}\right\}_{s \in S} \subset \Lambda(\Omega)$ is any family such that $\operatorname{supp} f_{s} \cap \operatorname{supp} f_{s^{\prime}}=$ $\varnothing$ for distinct $s, s^{\prime} \in S$. We define $\sum_{s \in S} f_{s} \in \Lambda(\Omega)$ in the following manner. Let $D_{0}=\bigcup_{s \in S} \operatorname{supp} f_{s}, D=D_{0} \cup \operatorname{int}\left(\Omega \backslash D_{0}\right)$ ('int' denotes the interior of a set) and $u: D \rightarrow \mathbb{R}_{+} \cup$ Card be given by $u(x)=f_{s}(x)$ for $x \in \operatorname{supp} f_{s}(s \in S)$ and $u(x)=0$ for $x \in \operatorname{int}\left(\Omega \backslash D_{0}\right)$. It is clear that $D$ is open and dense in $\Omega$ and $u$ is continuous. Now by Lemma 14.1, $u$ may be (uniquely) continuously extended to a member of $\Lambda(\Omega)$, denoted by $\sum_{s \in S} f_{s}$. One may check that in that case $\sum_{s \in S} f_{s}=\sup _{\Lambda(\Omega)}\left\{\sum_{s \in S_{0}} f_{s}: S_{0} \in \mathcal{P}_{f}(S)\right\}$.

15.1. Lemma. Let $\left\{f_{n}\right\}_{n=1}^{\infty} \subset \Lambda(\Omega)$ and $u, v: \Omega \rightarrow \mathbb{R}_{+} \cup$ Card be given by $u(x)=\inf _{n \geqslant 1} f_{n}(x)$ and $v(x)=\sup _{n \geqslant 1} f_{n}(x)(x \in \Omega)$. There are open dense subsets $U$ and $V$ of $\Omega$ such that $\left.u\right|_{U}$ and $\left.v\right|_{V}$ are continuous.

Proof. Since the proofs for $u$ and $v$ differ, we shall present both of them. We start with $u$ for which the proof is simpler. Let $U_{0}=u^{-1}\left(\mathbb{R}_{+}\right)$and for $\alpha \in \operatorname{Card}_{\infty}$ let $U_{\alpha}=\operatorname{int} u^{-1}(\{\alpha\})$. Since $U_{0}=\bigcup_{n=1}^{\infty} f_{n}^{-1}\left(\mathbb{R}_{+}\right), U_{0}$ is open. Now a function $u^{\prime}: \Omega \rightarrow \mathbb{R}_{+}$given by $u^{\prime}(x)=u(x)$ for $x \in U_{0}$ and $u^{\prime}(x)=0$ otherwise is Borel (because on $U_{0}$ it coincides with the infimum of a sequence of continuous functions taking values in $[0, \infty]$, after suitable change of $f_{n}$ 's). Thus, according to Lemma 14.2, there is a dense open subset $U^{\prime}$ of $\Omega$ such that $\left.u^{\prime}\right|_{U^{\prime}}$ is continuous. Consequently, $\left.u\right|_{U_{1}}$ is continuous where $U_{1}=U_{0} \cap U^{\prime}$ is open and dense in $U_{0}$. We see that $U=U_{1} \cup \bigcup_{\alpha \in \operatorname{Card}_{\infty}} U_{\alpha}$ is open and $\left.u\right|_{U}$ is continuous. To show that $U$ is dense in $\Omega$, it remains to check that the set $G=\operatorname{int}\left[\Omega \backslash\left(U_{0} \cup\right.\right.$ $\left.\left.\bigcup_{\alpha \in \operatorname{Card}_{\infty}} U_{\alpha}\right)\right]$ is empty. Suppose, for the contrary, that $G \neq \varnothing$. Note that $G$ is clopen and $u(G) \subset \operatorname{Card}_{\infty}$. Let $\alpha=\min u(G) \geqslant \aleph_{0}$. We conclude from the definition of $u$ that $f_{n}(x) \geqslant \alpha$ for all $x \in G$ and $n \geqslant 1$. What is more, there is $x_{0} \in G$ such that $u\left(x_{0}\right)=\alpha$ and there exists $m \geqslant 1$ with $u\left(x_{0}\right)=f_{m}\left(x_{0}\right)$. Since $\alpha$ is an isolated point of 
$f_{m}(G)$, the set $G_{0}=f_{m}^{-1}(\{\alpha\}) \cap G$ is clopen (and nonempty). We see that then $u(x)=\alpha$ for each $x \in G_{0}$ and hence $G_{0} \subset U_{\alpha}$ which denies the definition of $G$. This finishes the proof for $u$.

To show the assertion for $v$, we begin similarly: let $F=v^{-1}\left(I_{\aleph_{0}}\right)$ and $V_{\infty}=\bigcup_{\alpha \in \operatorname{Card}_{\infty}} \operatorname{int} v^{-1}(\{\alpha\})$. The set $F$ is closed since $F=$ $\bigcap_{n=1}^{\infty} f_{n}^{-1}\left(I_{\aleph_{0}}\right)$. We claim that

$$
F \cup \operatorname{cl} V_{\infty}=\Omega
$$

('cl' stands for the closure of a set). Again, we argue by contradiction. Suppose that the set $D=\Omega \backslash\left(F \cup \mathrm{cl} V_{\infty}\right)$ is nonempty. Since $D$ is open, there is a clopen set $G \neq \varnothing$ such that $G \subset D$. Notice that $v(G) \subset \operatorname{Card}_{\infty} \backslash\left\{\aleph_{0}\right\}$. Let $\gamma$ be the first infinite cardinal number such that

$$
\operatorname{int}\left[G \cap v^{-1}\left(I_{\gamma}\right)\right] \neq \varnothing .
$$

Let $W$ be any nonempty clopen subset of $G \cap v^{-1}\left(I_{\gamma}\right)$. Let us show that

$$
\gamma=\sup \left\{\sup f_{n}(W): n \geqslant 1\right\}=\sup v(W)>\aleph_{0} .
$$

Put $\gamma^{\prime}=\sup \left\{\sup f_{n}(W): n \geqslant 1\right\}$. It is clear that $\gamma^{\prime} \leqslant \sup v(W) \leqslant \gamma$ (because $\left.v(W) \subset I_{\gamma}\right)$. On the other hand, by the definition of $v$, $v(x) \leqslant \gamma^{\prime}$ for each $x \in W$ which yields that $\gamma^{\prime}>\aleph_{0}$ (since $W \subset G$ ) and $W \subset v^{-1}\left(I_{\gamma^{\prime}}\right) \cap G$. We infer from this and the definition of $\gamma$ that $\gamma \leqslant \gamma^{\prime}$. This proves (15-3).

Now let $W_{0}$ be an arbitrary nonempty clopen subset of $G \cap v^{-1}\left(I_{\gamma}\right)$ (cf. (15-2)). Put $Z=W_{0} \cap \bigcup_{n=1}^{\infty} f_{n}^{-1}(\{\gamma\}) . Z$ is $\mathcal{F}_{\sigma}$ and, by Baire's theorem, int $Z=\varnothing$ (because, thanks to (15-3), int $\left[W_{0} \cap f_{n}^{-1}(\{\gamma\})\right] \subset$ $\operatorname{int} v^{-1}(\{\gamma\}) \subset V_{\infty}$ and $\left.W_{0} \cap V_{\infty}=\varnothing\right)$. An application of Lemma 14.2 shows that $\operatorname{int}(\operatorname{cl} Z)=\varnothing$. This implies that there is a nonempty clopen set $W \subset W_{0} \backslash Z$. We conclude from the definition of $Z$ that $f_{n}(x)<\gamma$ for any $x \in W$ and $n \geqslant 1$. But since $W$ is compact, $f_{n}$ assumes its maximum on $W$ and consequently $\gamma_{n}:=\max \left(\aleph_{0}, \sup f_{n}(W)\right)<\gamma$. Now by (15-3),

$$
\sup _{n \geqslant 1} \gamma_{n}=\gamma
$$

Further, by the minimality of $\gamma$, each of the sets $G_{n}=G \cap v^{-1}\left(I_{\gamma_{n}}\right)$ has empty interior. Moreover, $G_{n}$ 's are closed $\left(G_{n}=G \cap \bigcap_{k=1}^{\infty} f_{k}^{-1}\left(I_{\gamma_{n}}\right)\right)$. Consequently, another applications of Baire's theorem and Lemma 14.2 give $\operatorname{int}\left[\operatorname{cl}\left(G_{\infty}\right)\right]=\varnothing$ where $G_{\infty}=\bigcup_{n=1}^{\infty} G_{n}$. But $G_{\infty}=G \cap v^{-1}\left(I_{\gamma} \backslash\right.$ $\{\gamma\}$ ) (by (15-4)). Finally, by (15-2), we obtain

$\operatorname{int}\left[G \cap v^{-1}(\{\gamma\})\right]=\operatorname{int}\left(G \cap v^{-1}\left(I_{\gamma}\right) \backslash G_{\infty}\right) \supset \operatorname{int}\left[G \cap v^{-1}\left(I_{\gamma}\right)\right] \backslash \operatorname{cl} G_{\infty} \neq \varnothing$ which denies the fact that $G \cap V_{\infty}=\varnothing$. This finishes the proof of $(15-1)$.

Relation (15-1) means that the set $E=\Omega \backslash \mathrm{cl} V_{\infty}$ is contained in $F$ and consequently $v(E) \subset I_{\aleph_{0}}$. Observe that $E$ is clopen and $I_{\aleph_{0}}$ is both 
homeomorphic and order-isomorphic to $[0,1]$. Therefore $\left.v\right|_{E}$ is Borel and by Lemma 14.2 there is an open dense subset $V_{0}$ of $E$ such that $\left.v\right|_{V_{0}}$ is continuous. To end the proof, put $V=V_{0} \cup V_{\infty}$.

Now assume $\left(f_{n}\right)_{n=1}^{\infty}$ is a sequence of members of $\Lambda(\Omega)$. Let $v: \Omega \ni$ $x \mapsto \sum_{n=1}^{\infty} f_{n}(x) \in \mathbb{R}_{+} \cup$ Card. (The series $\sum_{n=1}^{\infty} f_{n}(x)$ is understood as the supremum of its partial sums.) It is clear that $v\left(\Omega_{I}\right) \subset$ Card and $v\left(\Omega_{I I I}\right) \subset\{0\} \cup \operatorname{Card}_{\infty}$. By Lemma 15.1, there is an open dense subset $D$ of $\Omega$ such that $\left.v\right|_{D}$ is continuous. Consequently, thanks to Lemma 14.1 (page 40), there is a unique $\tilde{v} \in \Lambda(\Omega)$ which extends $\left.v\right|_{D}$. This unique extension $\tilde{v}$ will be denoted by $\sum_{n=1}^{\infty} f_{n}$. One may check that $\sum_{n=1}^{\infty} f_{n}=\sup _{\Lambda(\Omega)}\left\{\sum_{k=1}^{n} f_{k}: n \geqslant 1\right\}$.

Now let $\mathbb{A} \in \mathcal{C D D}_{N}$. Put

$$
s(\mathbb{A})=\mathbb{J} \boxminus\left(\mathbb{E}_{0}^{I}(\mathbb{A}) \boxplus \mathbb{E}_{0}^{I I}(\mathbb{A}) \boxplus \mathbb{E}_{0}^{I I I}(\mathbb{A})\right) .
$$

Since $s(\mathbb{A}) \leqslant^{s} \mathbb{J}, s(\mathbb{A})$ corresponds to a unique central projection $z_{\mathbb{A}} \in \mathcal{M}^{\prime}(\boldsymbol{J})$. There is a unique clopen set, denoted by $\operatorname{supp}_{\Omega} \mathbb{A}$, in $\Omega$ whose characteristic function coincides with $\Psi\left(z_{\mathbb{A}}\right)$. It is clear that for $\mathbb{A}, \mathbb{B} \in \mathcal{C D D}_{N}, \mathbb{A} \ll \mathbb{B} \Longleftrightarrow \operatorname{supp}_{\Omega} \mathbb{A} \subset \operatorname{supp}_{\Omega} \mathbb{B}$; and $\mathbb{A} \perp_{u} \mathbb{B} \Longleftrightarrow \operatorname{supp}_{\Omega} \mathbb{A} \cap \operatorname{supp}_{\Omega} \mathbb{B}=\varnothing$. When $\mathbb{X}, \mathbb{Y} \in \mathcal{S \mathcal { M }}_{N}$ are such that $\mathbb{X} \ll \mathbb{Y}, u=\frac{\mathrm{d} \mathbb{X}}{\mathrm{d} \mathbb{Y}}$ is defined on $\Omega_{I I}$ and real-valued on an open dense subset $D$ of $\Omega_{I I}$. Extending $\left.u\right|_{D}$ to a continuous function of $\Omega$ into $I_{\aleph_{0}}$ by putting zero on $\Omega_{I} \cup \Omega_{I I I}$ and applying Lemma 14.1, we may consider $\frac{d \mathbb{X}}{d \mathbb{Y}}$ as a member of $\Lambda(\Omega)$, as it is done in this section. Under such an understanding,

$$
\begin{aligned}
&\left\{\frac{\mathrm{d} \mathbb{X}}{\mathrm{d} \mathbb{Y}}: \mathbb{X} \in \mathcal{S \mathcal { M }}_{N},\right.\mathbb{X} \ll \mathbb{Y}\}=\{u \in \Lambda(\Omega): \\
&\left.\operatorname{supp} u \subset \operatorname{supp}_{\Omega} \mathbb{Y}, u^{-1}\left(\mathbb{R}_{+}\right) \text {is dense in } \Omega\right\}
\end{aligned}
$$

(by Theorem 14.3). Since the addition is continuous on $I_{\aleph_{0}}, \frac{\mathrm{d}\left(\mathbb{X}^{\prime} \oplus \mathbb{X}^{\prime \prime}\right)}{\mathrm{d} \mathbb{Y}}=$ $\frac{d \mathbb{X}^{\prime}}{d \mathbb{Y}}+\frac{d \mathbb{X}^{\prime \prime}}{d \mathbb{Y}}$ whenever $\mathbb{X}^{\prime}, \mathbb{X}^{\prime \prime} \ll \mathbb{Y}$.

Everywhere below $j_{E}$ denotes the characteristic function of a set $E \subset \Omega$.

15.2. Theorem. Let $\mathbb{T} \in \mathcal{S M}_{N}$ be such that $\aleph_{0} \odot \mathbb{T}=\mathbb{J}_{I I}$ (there exists such $\mathbb{T})$. There is a unique assignment $\Phi_{\mathbb{T}}: \operatorname{CDD}_{N} \rightarrow \Lambda(\Omega)$ such that

(D0) $\Phi_{\mathbb{T}}(\mathbb{T})=j_{\Omega_{I I}}, \Phi_{\mathbb{T}}\left(\mathbb{J}_{I}\right)=j_{\Omega_{I}}$ and $\Phi_{\mathbb{T}}\left(\mathbb{J}_{I I I}\right)=\aleph_{0} \cdot j_{\Omega_{I I I}}$,

(D1) $\operatorname{supp} \Phi_{\mathbb{T}}(\mathbb{A}) \subset \operatorname{supp}_{\Omega} \mathbb{A}$ for each $\mathbb{A} \in \mathcal{C D D}_{N}$,

(D2) $\Phi_{\mathbb{T}}(\alpha \odot \mathbb{A})=\alpha \cdot \Phi_{\mathbb{T}}(\mathbb{A})$ for any $\alpha \in$ Card and $\mathbb{A} \in \mathcal{C D D}_{N}$,

(D3) whenever $\left\{\mathbb{A}^{(s)}\right\}_{s \in S} \subset \mathcal{C D D}_{N}$ is a regular family (cf. (D1) and notes on page 44),

$$
\Phi_{\mathbb{T}}\left(\bigoplus_{s \in S} \mathbb{A}^{(s)}\right)=\sum_{s \in S} \Phi_{\mathbb{T}}\left(\mathbb{A}^{(s)}\right)
$$


(D4) whenever $\left(\mathbb{A}^{(n)}\right)_{n=1}^{\infty} \subset \mathcal{C D} \mathcal{D}_{N}$ is such that $\bigoplus_{n=1}^{\infty} \mathbb{A}^{(n)} \in \mathcal{S M}_{N}$ (see notes on page 46$)$,

$$
\Phi_{\mathbb{T}}\left(\bigoplus_{n=1}^{\infty} \mathbb{A}^{(n)}\right)=\sum_{n=1}^{\infty} \Phi_{\mathbb{T}}\left(\mathbb{A}^{(n)}\right)
$$

What is more, $\Lambda(\Omega)$ is order-complete and $\Phi_{\mathbb{T}}$ has further properties (below $\mathbb{A}, \mathbb{B} \in \mathcal{C D} \mathcal{D}_{N}$ ):

$\left(\mathrm{D} 1^{\prime}\right) \operatorname{supp} \Phi_{\mathbb{T}}(\mathbb{A})=\operatorname{supp}_{\Omega} \mathbb{A} ;$ in particular, $\mathbb{A} \ll \mathbb{B}\left(\mathbb{A} \perp_{u} \mathbb{B}\right)$ iff $\operatorname{supp}_{\Omega} \Phi_{\mathbb{T}}(\mathbb{A}) \subset \operatorname{supp}_{\Omega} \Phi_{\mathbb{T}}(\mathbb{B})\left(\operatorname{supp}_{\Omega} \Phi_{\mathbb{T}}(\mathbb{A}) \cap \operatorname{supp}_{\Omega} \Phi_{\mathbb{T}}(\mathbb{B})=\varnothing\right)$,

(D4') for any sequence $\left(\mathbb{A}^{(n)}\right)_{n=1}^{\infty} \subset \mathcal{C D} \mathcal{D}_{N}$,

$$
\Phi_{\mathbb{T}}\left(\bigoplus_{n=1}^{\infty} \mathbb{A}^{(n)}\right)=\sum_{n=1}^{\infty} \Phi_{\mathbb{T}}\left(\mathbb{A}^{(n)}\right)
$$

in particular,

$$
\Phi_{\mathbb{T}}(\mathbb{A} \oplus \mathbb{B})=\Phi_{\mathbb{T}}(\mathbb{A})+\Phi_{\mathbb{T}}(\mathbb{B}),
$$

(D5) $\mathbb{A} \leqslant \mathbb{B} \Longleftrightarrow \Phi_{\mathbb{T}}(\mathbb{A}) \leqslant \Phi_{\mathbb{T}}(\mathbb{B})$,

(D6) $\mathbb{A} \leqslant \leqslant^{s} \mathbb{B} \Longleftrightarrow \Phi_{\mathbb{T}}(\mathbb{A})=\Phi_{\mathbb{T}}(\mathbb{B}) \cdot j_{E}$ for some clopen set $E \subset \Omega$,

(D7) for every $\mathbb{X} \in \mathcal{S} \mathcal{M}_{N}, \Phi_{\mathbb{T}}(\mathbb{X})=\frac{\mathrm{d} \mathbb{X}}{\mathrm{d} \mathbb{T}}$,

(D8) for every $u \in \Lambda(\Omega)$ there is a unique $\mathbb{X} \in \mathcal{C D D}_{N}$ such that $\Phi_{\mathbb{T}}(\mathbb{X})=u$.

Proof. Let us start with uniqueness of $\Phi_{\mathbb{T}}$. First of all, for $\mathbb{A} \in \mathcal{S} \mathcal{M}_{N}$, $s(\mathbb{A})=\aleph_{0} \odot \mathbb{A}$ and hence $\operatorname{supp}_{\Omega} \mathbb{A}$ coincides with $\operatorname{supp}_{\Omega_{I I}} \mathbb{A}$ introduced in the previous section. Therefore (D0), (D1) and (D4) combined with Theorem 14.3 (page 41 ) yield that $\Phi_{\mathbb{T}}(\mathbb{A})=\frac{\mathrm{d} \mathbb{A}}{\mathrm{d} \mathbb{T}}$ for $\mathbb{A} \in \mathcal{S \mathcal { M }}_{N}$ (notice that $\mathbb{A} \ll \mathbb{T}$ for every such $\mathbb{A}$ ). Further, we infer from (D0) and (D2) that $\Phi_{\mathbb{T}}\left(\mathbb{J}_{I I}\right)=\aleph_{0} \cdot j_{\Omega_{I I}}$ and consequently, by (D3) and (D0),

$$
\Phi_{\mathbb{T}}(\mathbb{J})=j_{\Omega_{I}}+\aleph_{0} \cdot j_{\Omega_{I I} \cup \Omega_{I I I}} .
$$

Now if $\mathbb{X} \leqslant^{s} \mathbb{J},(\mathrm{D} 3)$ implies that $\Phi_{\mathbb{T}}(\mathbb{I})=\Phi_{\mathbb{T}}(\mathbb{X})+\Phi_{\mathbb{T}}(\mathbb{Y})$ with $\mathbb{Y}=$ $\mathbb{J} \boxminus \mathbb{X}$. What is more, $\operatorname{supp}_{\Omega} \mathbb{X} \cap \operatorname{supp}_{\Omega} \mathbb{Y}=\varnothing$ from which we conclude, thanks to $(\mathrm{D} 1)$, that $\Phi_{\mathbb{T}}(\mathbb{X})=\Phi_{\mathbb{T}}(\mathbb{J}) \cdot j_{\operatorname{supp}_{\Omega} \mathbb{X}}$. Finally, if $\mathbb{A} \in \mathcal{C D} \mathcal{D}_{N}$ is arbitrary, the above notices combined with (D3) and (D2) give

$$
\Phi_{\mathbb{T}}(\mathbb{A})=\frac{\mathrm{d} \mathbb{E}_{s m}(\mathbb{A})}{\mathrm{d} \mathbb{T}}+\sum_{(i, \alpha) \in \Upsilon_{*}} \alpha \cdot \Phi_{\mathbb{T}}(\mathbb{J}) \cdot j_{\operatorname{supp}_{\Omega} \mathbb{E}_{\alpha}^{i}(\mathbb{A})} .
$$

To establish the existence of $\Phi_{\mathbb{T}}$ together with all suitable properties, define $\Phi_{\mathbb{T}}(\mathbb{A})$ by (15-9) with $\Phi_{\mathbb{T}}(\mathbb{J})$ given by (15-8). Observe that (D0), (D1'), (D2) and (D7) are fulfilled. We shall show now (15-7). We shall apply here calculations presented in Example 11.7 (page 28). Under 
the notation of that example, (15-9) and (11-6) give

$$
\begin{aligned}
\Phi_{\mathbb{T}}(\mathbb{A} \oplus \mathbb{B})=\frac{\mathrm{d} \mathbb{E}_{s m, 0}}{\mathrm{~d} \mathbb{T}}+\frac{\mathrm{d} \mathbb{E}_{s m, 1}}{\mathrm{~d} \mathbb{T}}+\frac{\mathrm{d} \mathbb{E}_{0, s m}}{\mathrm{~d} \mathbb{T}}+\frac{\mathrm{d} \mathbb{E}_{1, s m}}{\mathrm{~d} \mathbb{T}} \\
+\sum_{(i, \alpha, \beta) \in \Upsilon_{\#}^{2}}(\alpha+\beta) \cdot\left(\Phi_{\mathbb{T}}(\mathbb{J}) \cdot j_{\left.\operatorname{supp}_{\Omega} \mathbb{E}_{\alpha, \beta}^{i}\right) .} .\right.
\end{aligned}
$$

Further, it follows from Theorem 14.3 (page 41) that

$$
\begin{aligned}
& \frac{d \mathbb{E}_{s m}(\mathbb{A})}{\mathrm{d} \mathbb{T}}=\frac{d \mathbb{E}_{s m, 0}}{\mathrm{~d} \mathbb{T}}+\frac{\mathrm{d} \mathbb{E}_{s m, 1}}{\mathrm{~d} \mathbb{T}}+\sum_{\alpha \in \operatorname{Card}_{\infty}} \frac{\mathrm{d} \mathbb{E}_{s m, \alpha}}{\mathrm{d} \mathbb{T}}, \\
& \frac{\mathrm{d} \mathbb{E}_{s m}(\mathbb{B})}{\mathrm{d} \mathbb{T}}=\frac{\mathrm{d} \mathbb{E}_{0, s m}}{\mathrm{~d} \mathbb{T}}+\frac{\mathrm{d} \mathbb{E}_{1, s m}}{\mathrm{~d} \mathbb{T}}+\sum_{\alpha \in \operatorname{Card}_{\infty}} \frac{\mathrm{d} \mathbb{E}_{\alpha, s m}}{\mathrm{~d} \mathbb{T}} .
\end{aligned}
$$

On the other hand, $(i, \alpha) \in \Upsilon_{*}, \mathbb{E}_{\alpha}^{i}(\mathbb{A})=\boxplus_{\beta \in \Lambda_{i}} \mathbb{E}_{\alpha, \beta}^{i}$ and $\mathbb{E}_{\alpha}^{i}(\mathbb{B})=$ $\boxplus_{\beta \in \Lambda_{i}} \mathbb{E}_{\beta, \alpha}^{i}$ which means that

$$
j_{\operatorname{supp}_{\Omega} \mathbb{E}_{\alpha}^{i}(\mathbb{A})}=\sum_{\beta \in \Lambda_{i}} j_{\operatorname{supp}_{\Omega} \mathbb{E}_{\alpha, \beta}^{i}} \text { and } \quad j_{\operatorname{supp}_{\Omega} \mathbb{E}_{\alpha}^{i}(\mathbb{B})}=\sum_{\beta \in \Lambda_{i}} j_{\operatorname{supp}_{\Omega} \mathbb{E}_{\beta, \alpha}^{i}} .
$$

Substituting the above formulas in the ones for $\Phi_{\mathbb{T}}(\mathbb{A})$ and $\Phi_{\mathbb{T}}(\mathbb{B})$, we see that (15-7) is satisfied.

Now let $g$ be an arbitrary member of $\Lambda(\Omega)$. For $(i, \alpha) \in \Upsilon_{*}$ let $U_{\alpha}^{i}=\Omega_{i} \cap \operatorname{int} g^{-1}(\{\alpha\})$ and let $U_{1}^{I I}$ be the closure of $g^{-1}\left(\mathbb{R}_{+} \backslash\{0\}\right) \cap \Omega_{I I}$. Since $\Omega$ is extremely disconnected, the sets $U_{\alpha}^{i}$ 's (with $(i, \alpha) \in \Upsilon$ ) are clopen and pairwise disjoint. The arguments used in the proof of Lemma 15.1 show that their union is dense in $\Omega$. This implies that there is a partition of unity $\left\{\mathbb{E}_{\alpha}^{i}\right\}_{(i, \alpha) \in \Upsilon} \subset \mathcal{C D} \mathcal{D}_{N}$ such that $\operatorname{supp}_{\Omega} \mathbb{E}_{\alpha}^{i}=$ $U_{\alpha}^{i}$ for every $(i, \alpha) \in \Upsilon$. Moreover, thanks to (15-6), there is $\mathbb{E}_{s m} \in$ $\mathcal{S \mathcal { M }}_{N}$ such that $\frac{\mathrm{dE} \mathbb{E}_{s m}}{\mathrm{dT}}=g \cdot j_{U_{1}^{I I}}$. The latter implies that $\operatorname{supp}_{\Omega} \mathbb{E}_{s m}=$ $\operatorname{supp}_{\Omega} \mathbb{E}_{1}^{I I}$ and hence $\mathbb{E}_{1}^{I I}=\aleph_{0} \odot \mathbb{E}_{s m}$. Now the formulas $\mathbb{E}_{\alpha}^{i}(\mathbb{A}):=\mathbb{E}_{\alpha}^{i}$ and $\mathbb{E}_{s m}(\mathbb{A}):=\mathbb{E}_{s m}$ well defines $\mathbb{A} \in \mathcal{C D D}_{N}$ such that $\Phi_{\mathbb{T}}(\mathbb{A})=g$. Further, if $\Phi_{\mathbb{T}}(\mathbb{B})=g$ and $V_{\alpha}^{i}=\operatorname{supp}_{\Omega} \mathbb{E}_{\alpha}^{i}(\mathbb{B})((i, \alpha) \in \Upsilon)$, then $V_{\alpha}^{i} \subset$ $U_{\alpha}^{i}$ for $(i, \alpha) \in \Upsilon$, by (15-9). But the union of all $V_{\alpha}^{i}$ 's is dense in $\Omega$ and $U_{\alpha}^{i} \backslash V_{\alpha}^{i}$ is open. We infer from this that $V_{\alpha}^{i}=U_{\alpha}^{i}$ and consequently $\frac{\mathrm{d} \mathbb{B}}{\mathrm{d} \mathbb{T}}=\frac{\mathrm{d} \mathbb{A}}{\mathrm{d} \mathbb{T}}$ and $\mathbb{B}=\mathbb{A}$. This shows (D8).

We are now able to prove easily (D5) and (D8). Indeed, if $\mathbb{A} \leqslant \mathbb{B}$, then $\mathbb{B}=\mathbb{A} \oplus \mathbb{X}$ for some $\mathbb{X}$ and then, by $(15-7), \Phi_{\mathbb{T}}(\mathbb{B})=\Phi_{\mathbb{T}}(\mathbb{A})+$ $\Phi_{\mathbb{T}}(\mathbb{X}) \geqslant \Phi_{\mathbb{T}}(\mathbb{A})$. Conversely, if $\Phi_{\mathbb{T}}(\mathbb{A}) \leqslant \Phi_{\mathbb{T}}(\mathbb{B})$, there is $g \in \Lambda(\Omega)$ (see Corollary 15.3 below) for which $\Phi_{\mathbb{T}}(\mathbb{B})=\Phi_{\mathbb{T}}(\mathbb{A})+g$. We know from the previous argument that $g=\Phi_{\mathbb{T}}(\mathbb{X})$ for some $\mathbb{X} \in \mathcal{C D} \mathcal{D}_{N}$. Consequently, $\Phi_{\mathbb{T}}(\mathbb{B})=\Phi_{\mathbb{T}}(\mathbb{A} \oplus \mathbb{X})$ and by $(\mathrm{D} 8), \mathbb{B}=\mathbb{A} \oplus \mathbb{X}$ and we are done.

We have shown that $\Phi_{\mathbb{T}}$ is a bijective order isomorphism. This implies that $\Lambda(\Omega)$ is order-complete (by Theorem 4.2, page 9) and for 
every nonempty set $\left\{\mathbb{A}^{(s)}\right\}_{s \in S} \subset \mathcal{C D} \mathcal{D}_{N}$,

$$
\begin{aligned}
\Phi_{\mathbb{T}}\left(\bigvee\left\{\bigoplus_{s \in S_{0}} \mathbb{A}^{(s)}: S_{0} \in \mathcal{P}_{f}(S)\right\}\right)= & \\
& =\sup _{\Lambda(\Omega)}\left\{\sum_{s \in S_{0}} \Phi_{\mathbb{T}}\left(\mathbb{A}^{(s)}\right): S_{0} \in \mathcal{P}_{f}(S)\right\} .
\end{aligned}
$$

But this and (AO6) (page 29) imply (D3), (D4) and (D4'). Point (D6) is left for the reader.

Every topological space homeomorphic to $\Omega$ is called by us an underlying model space for $\mathcal{C D D}_{N}$. In the sequel we shall show that underlying model spaces for $\mathcal{C D} \mathcal{D}_{N}$ and $\mathcal{C D} \mathcal{D}_{N^{\prime}}$ are homeomorphic for any natural numbers $N$ and $N^{\prime}$. We shall also propose a simplified form of them.

Let us now list a few basic consequences of Theorem 15.2. Some of them were announced in Section 12. For simplicity, we fix $\mathbb{T} \in \mathcal{S M}_{N}$ such that $\aleph_{0} \odot \mathbb{T}=\mathbb{J}_{I I}$ and for each $\mathbb{A} \in \mathcal{C D} \mathcal{D}_{N}, \widehat{\mathbb{A}}$ will denote $\Phi_{\mathbb{T}}(\mathbb{A})$. Since $\Lambda(\Omega)$ is order-complete, for every nonempty set $\left\{f_{s}\right\}_{s \in S} \subset \Lambda(\Omega)$, $\bigvee_{s \in S} f_{s}$ and $\bigwedge_{s \in S} f_{s}$ will stand for, respectively, $\sup _{\Lambda(\Omega)}\left\{f_{s}: s \in S\right\}$ and $\inf _{\Lambda(\Omega)}\left\{f_{s}: s \in S\right\}$.

15.3. Corollary. $(\mathbb{B} \ominus \mathbb{X})_{\Delta} \vee(\mathbb{X} \ominus \mathbb{A})_{\Delta} \leqslant(\mathbb{B} \ominus \mathbb{A})_{\Delta}$ provided $\mathbb{A} \leqslant \mathbb{X} \leqslant \mathbb{B}$.

Proof. It suffices to prove a counterpart of the corollary in the class $\Lambda(\Omega)$. Let $f, g \in \Lambda(\Omega)$ be such that $f \leqslant g$. The set $D_{0}=\{x \in$ $\Omega: f(x)<f(y)$ or $\left.f(y) \in \mathbb{R}_{+}\right\}$is open in $\Omega$ and there is a unique function $u_{0}: D_{0} \rightarrow \mathbb{R}_{+} \cup$ Card such that $g(x)=u_{0}(x)+f(x)$ for every $x \in D_{0}$. It may be easily seen that $u_{0}$ is continuous. Let $D(f, g)=D_{0} \cup$ $\operatorname{int}\left(\Omega \backslash D_{0}\right)$ and $u \in \Lambda(\Omega)$ be a unique continuous function (guaranteed by Lemma 14.1, page 40) such that $u(x)=u_{0}(x)$ for $x \in D_{0}$ and $u(x)=0$ for $x \in D(f, g) \backslash D_{0}$. We see that $g=f+u$ on $D(f, g)$ and hence $g=f+u$ on $\Omega$. It is easily seen that $u$ is the least member of $(\Lambda(\Omega), \leqslant)$ with the latter property. We shall denote this $u$ by $(g-f)_{\Delta}$. It is clear that

$$
(\widehat{\mathbb{B} \ominus \mathbb{A}})_{\Delta}=(\widehat{\mathbb{B}}-\widehat{\mathbb{A}})_{\Delta}
$$

whenever $\mathbb{A} \leqslant \mathbb{B}$. Thus, we need to check that $(h-g)_{\Delta} \vee(g-f)_{\Delta} \leqslant$ $(h-f)_{\Delta}$ if only $f \leqslant g \leqslant h$. It suffices to check suitable inequality on a dense subset of $\Omega$. We leave this as a simple exercise that for $x \in D(f, g) \cap D(g, h) \cap D(f, h)$ it is fulfilled.

15.4. Remark. Using the same idea as in the proof of Corollary 15.3, one may show that whenever $\mathbb{A}, \mathbb{B} \in \mathcal{C D} \mathcal{D}_{N}$ are such that $\mathbb{A} \leqslant \mathbb{B}$, then

$$
\begin{aligned}
{\left[\mathbb{B} \ominus(\mathbb{B} \ominus \mathbb{A})^{\nabla}\right]_{\Delta} \leqslant } & \leqslant[B( \\
& \left.\left.\leqslant[\mathbb{B} \ominus \mathbb{A})_{\Delta}\right]_{\Delta} \leqslant^{s} \mathbb{A} \leqslant(\mathbb{B} \ominus \mathbb{A})_{\Delta}\right]^{\nabla}=\left[\mathbb{B} \ominus(\mathbb{B} \ominus \mathbb{A})^{\nabla}\right]^{\nabla} .
\end{aligned}
$$


Recall that the Souslin number of a topological space $X$, denoted by $c(X)([8$, Problem 1.7.12]), is the least infinite cardinal number $\alpha$ such that every family of mutually disjoint nonempty open subsets of $X$ has power no greater than $\alpha$. Let us modify this by putting $c_{*}(\varnothing)=0$ and $c_{*}(X)=c(X)$ for nonempty topological spaces $X$. It turns out that the modified Souslin numbers of certain clopen subsets of $\Omega$ may be used to give the formula for $\operatorname{dim}(\mathbb{A})$ if only this dimension is infinite. Namely,

15.5. Proposition. Let $\mathbb{A} \in \mathcal{C D} \mathcal{D}_{N}$ and $f=\widehat{\mathbb{A}}$. Let $U_{1}^{I I}$ be the closure of $f^{-1}\left(\mathbb{R}_{+} \backslash\{0\}\right) \cap \Omega_{I I}$ and for $(i, \alpha) \in \Upsilon_{*}$ let $U_{\alpha}^{i}=\Omega_{i} \cap \operatorname{int} f^{-1}(\{\alpha\})$. Then

$$
\aleph_{0} \cdot \operatorname{dim}(\mathbb{A})=\sum_{(i, \alpha) \in \Upsilon} \alpha \cdot c_{*}\left(U_{\alpha}^{i}\right) .
$$

Proof. In extremely disconnected spaces, the closures of two disjoint open sets are disjoint as well. Consequently, whenever $E$ is a clopen subset of $\Omega, c(E)$ is the least infinite cardinal $\alpha$ such that every family of pairwise disjoint nonempty clopen sets has power no greater than $\alpha$. Since clopen sets correspond to $N$-tuples $\mathbb{A}$ such that $\mathbb{A} \leqslant^{s} \mathbb{J}$, the assertion follows from the argument used in (ST17) (page 35). The details are left for the reader (cf. the proof of point (D8) in Theorem 15.2).

15.6. Remark. It is worthwhile to mention that it is impossible to recognize $N$-tuples whose representatives act on finite-dimensional spaces by means of corresponding to them members of $\Lambda(\Omega)$, unless we distinguish some special subsets of $\Omega$, as it is done in the next section. To convince of that, it suffices to note that $\widehat{\mathbb{A}}$ is the characteristic function of a one-point subset of $\Omega_{I}$ if e.g. $A=(T, \ldots, T) \in \mathrm{CDD}_{N}$ where $T$ is either the identity operator on $\mathbb{C}$ or a unilateral shift on $l^{2}$.

We shall now prove a useful

15.7. Lemma. (A) For every clopen nonempty set $E \subset \Omega$ there is a family $\left\{E_{s}\right\}_{s \in S}$ of pairwise disjoint clopen nonempty sets such that $c\left(E_{s}\right)=\aleph_{0}$ for every $s \in S$ and $\bigcup_{s \in S} E_{s}$ is a dense subset of $E$.

(B) Let $\left\{f_{s}\right\}_{s \in S}$ be a nonempty set of members of $\Lambda(\Omega)$ and let $u=$ $\bigwedge_{s \in S} f_{s}$ and $v=\bigvee_{s \in S} f_{s}$. For every clopen nonempty set $E \subset \Omega$ with $c(E)=\aleph_{0}$ there are a nonempty set $S(E) \in \mathcal{P}_{\omega}(S)$ and an open dense subset $D(E)$ of $E$ with the following property. Whenever $S^{\prime} \supset S(E)\left(S^{\prime} \subset S\right)$ and $x \in D(E)$,

$$
u(x)=\inf _{s \in S^{\prime}} f_{s}(x)
$$

and if, in addition, $v(E) \subset I_{\aleph_{0}}$, then also

$$
v(x)=\sup _{s \in S^{\prime}} f_{s}(x) \text {. }
$$


Proof. (A): Let $\mathcal{E}=\left\{E_{s}\right\}_{s \in S}$ be a maximal family of pairwise disjoint nonempty clopen sets such that $c\left(E_{s}\right)=\aleph_{0}$ and $E_{s} \subset E$ for every $s \in S$. Let $D=E \backslash \operatorname{cl}\left(\bigcup_{s \in S} E_{s}\right)$. We have to show that $D$ is empty. But this follows from Proposition 9.10 (page 24). Indeed, we infer from this result that every nonempty clopen subset of $\Omega$ contains a nonempty clopen set $G$ with $c(G)=\aleph_{0}$. Consequently, since $D$ is clopen and $\mathcal{E}$ is maximal, $D=\varnothing$.

(B): Let $U_{1}=\operatorname{cl} u^{-1}\left(\mathbb{R}_{+}\right) \cap E$ and $U_{\alpha}=\operatorname{int} u^{-1}(\{\alpha\}) \cap E$ for $\alpha \in$ $\operatorname{Card}_{\infty}$. We know (cf. the proof of Lemma 15.1, page 44) that the collection $\mathcal{U}=\left\{U_{\alpha}: \alpha \in \operatorname{Card}_{\infty} \cup\{1\}\right\}$ consists of pairwise disjoint clopen sets whose union is dense in $E$. Further, for each $\alpha \in \operatorname{Card}_{\infty}$ and $s \in S$ put $U_{\alpha, s}=U_{\alpha} \cap f_{s}^{-1}(\{\alpha\})$. Since $f_{s} \geqslant \alpha$ on $U_{\alpha}$ and $\alpha$ is an isolated point of Card $\backslash\{\beta \in$ Card: $\beta<\alpha\}, U_{\alpha, s}$ is clopen. It is clear that $\bigcup_{s \in S} U_{\alpha, s}$ is dense in $U_{\alpha}$. (Indeed, the set $G=U_{\alpha} \backslash \operatorname{cl}\left(\bigcup_{s \in S} U_{\alpha, s}\right)$ is clopen and $f_{s}(x) \geqslant \alpha^{+}$for any $x \in G$ and $s \in S$ and thus $u^{\prime} \in \Lambda(\Omega)$ given by $\left.u^{\prime}\right|_{G} \equiv \alpha^{+}$and $u^{\prime}=u$ on $\Omega \backslash G$ is such that $u^{\prime} \leqslant f_{s}(s \in S)$ which gives $u^{\prime} \leqslant u$ and consequently $G=\varnothing$.) Let ' $<$ ' be a well order on $S$ with the first element $s_{*}$. We define clopen sets $V_{\alpha, s}$ by transfinite induction as follows. Let $V_{\alpha, s_{*}}=U_{\alpha, s_{*}}$ and for any $s \in S \backslash\left\{s_{*}\right\}$,

$$
V_{\alpha, s}=U_{\alpha, s} \backslash \operatorname{cl}\left(\bigcup_{s^{\prime}<s} V_{\alpha, s^{\prime}}\right) \text {. }
$$

We see that $V_{\alpha, s} \subset U_{\alpha, s}$ and hence

$$
\left.u\right|_{V_{\alpha, s}}=\left.f_{s}\right|_{V_{\alpha, s}} .
$$

Further, the sets $V_{\alpha, s}(s \in S)$ are pairwise disjoint. Using transfinite induction one may check that $\operatorname{cl}\left(\bigcup_{s^{\prime}<s} V_{\alpha, s^{\prime}}\right)=\operatorname{cl}\left(\bigcup_{s^{\prime}<s} U_{\alpha, s^{\prime}}\right)$ for each $s \in S$ and thus

$$
\operatorname{cl}\left(\bigcup_{s \in S} V_{\alpha, s}\right)=U_{\alpha}
$$

Now we pass to the set $U_{1}$. By the definition, $U_{1}$ is clopen and $u\left(U_{1}\right) \subset$ $I_{\aleph_{0}}$. In what follows, we assume $U_{1}$ is nonempty. Let $g_{s}=f_{s} \wedge \aleph_{0}$. We naturally identify $I_{\aleph_{0}}$ with $[0, \infty]$. Let $\tau:[0, \infty] \ni x \mapsto \frac{x}{x+1} \in[0,1]$ (with convention that $\left.\frac{\infty}{\infty+1}=1\right)$. Put $u^{\prime}=\left.\tau \circ u\right|_{U_{1}} \in \mathcal{C}\left(U_{1},[0,1]\right)$ and $g_{s}^{\prime}=\left.\tau \circ g_{s}\right|_{U_{1}} \in \mathcal{C}\left(U_{1},[0,1]\right)$. Note that

$$
u^{\prime}=\bigwedge_{s \in S} g_{s}^{\prime} .
$$

Since $U_{1}$ is clopen in $\Omega$ and $\mathcal{C}(\Omega)$ is a $\mathcal{W}^{*}$-algebra, so is $\mathcal{C}\left(U_{1}\right)$. Further, we conclude from the fact that $c\left(U_{1}\right)=\aleph_{0}$ that $\mathcal{C}\left(U_{1}\right)$ is countably decomposable. Thus, it may be infered from [34, Theorem III.1.18] or [29, Proposition 1.18.1] that $\mathcal{C}\left(U_{1}\right)$ is isomorphic to $L^{\infty}(\mu)$ for some probabilistic space $(X, \mathfrak{M}, \mu)$. Under the isomorphism, $g_{s}^{\prime}$ and $u^{\prime}$ correspond to, respectively, $\xi_{s} \in L^{\infty}(\mu)$ and $w \in L^{\infty}(\mu)$. Consequently, 
$w=\inf _{L^{\infty}(\mu)}\left\{\xi_{s}: s \in S\right\}$ (by (15-13)). For a nonempty set $S_{0} \in \mathcal{P}_{\omega}(S)$ let $w_{S_{0}}: X \ni x \mapsto \inf _{s \in S_{0}} \xi_{s}(x) \in[0,1]$. Since $S_{0}$ is countable, $w_{S_{0}}$ is measurable and hence $w_{S_{0}} \in L^{\infty}(\mu)$. Let

$$
c=\inf \left\{\int_{X} w_{S_{0}} \mathrm{~d} \mu: S_{0} \in \mathcal{P}_{\omega}(S)\right\} .
$$

It is easily seen that there is $S_{1} \in \mathcal{P}_{\omega}(S)$ for which $c=\int_{X} w_{S_{1}} \mathrm{~d} \mu$. Now if $s$ is an arbitrary element of $S$, then $w_{S_{1} \cup\{s\}} \leqslant w_{S_{1}}$ and $\int_{X} w_{S_{1} \cup\{s\}} \mathrm{d} \mu \geqslant$ $c=\int_{X} w_{S_{1}} \mathrm{~d} \mu$. These imply that $w_{S_{1} \cup\{s\}}=w_{S_{1}}$ ( $\mu$-almost everywhere) and consequently $\xi_{s} \geqslant w_{S_{1}}$ in $L^{\infty}(\mu)$. The latter gives $w \geqslant$ $w_{S_{1}}=\inf _{L^{\infty}(\mu)}\left\{\xi_{s}: s \in S_{1}\right\}$ and therefore $w=w_{S_{1}}$ (in $L^{\infty}(\mu)$ ). In $\mathcal{C}\left(U_{1}\right)$ this is interpreted as $u^{\prime}=\bigwedge_{s \in S_{1}} g_{s}^{\prime}$ which is equivalent to $\left.u\right|_{U_{1}}=\left.\bigwedge_{s \in S_{1}} g_{s}\right|_{U_{1}}$. Now by Lemma 15.1 (page 44), $u(x)=\inf _{s \in S_{1}} g_{s}(x)$ for $x \in D_{1}$ where $D_{1}$ is an open dense subset of $U_{1}$. This implies that for each $x \in D_{1}(E):=D_{1} \cap u^{-1}\left(\mathbb{R}_{+}\right) \cap E$ there is $s_{x} \in S_{1}$ such that $g_{s_{x}}(x) \in \mathbb{R}_{+}$. Consequently, $g_{s_{x}}(x)=f_{s_{x}}(x)$ and hence

$$
u(x)=\inf _{s \in S_{1}} f_{s}(x)
$$

for $x \in D_{1}(E)$. Notice that $D_{1}(E)$ is dense in $U_{1}$.

Further, observe that the family $\left\{U_{1}\right\} \cup\left\{V_{\alpha, s}: s \in S, \alpha \in \operatorname{Card}_{\infty}\right\}$ constists of pairwise disjoint clopen subsets of $E$. Since $c(E)=\aleph_{0}$, the set $J:=\left\{(\alpha, s): s \in S, \alpha \in \operatorname{Card}_{\infty}, U_{\alpha, s} \neq \varnothing\right\}$ is countable (finite or not). Put $S(E)=S_{1} \cup\{s:(\alpha, s) \in J\}$ and $D(E)=D_{1}(E) \cup$ $\bigcup_{(\alpha, s) \in J} V_{\alpha, s}$. We see that $S(E) \in \mathcal{P}_{\omega}(S)$ and $D(E)$ is open and dense in $E$ (by (15-12) and the density of $D_{1}(E)$ in $U_{1}$ ). Take an arbitrary set $S^{\prime}$ such that $S(E) \subset S^{\prime} \subset S$. For each $x \in \Omega$ one has $\inf _{s \in S^{\prime}} f_{s}(x) \geqslant u(x)$. On the other hand, if $x \in D(E)$, then either $x \in D_{1}(E)$ or $x \in V_{\alpha, s}$ for some $(\alpha, s) \in J$. In the first case the inequality $\inf _{s \in S^{\prime}} f_{s}(x) \leqslant u(x)$ follows from (15-14), while in the second one from (15-11).

If we additionally assume that $v(E) \subset I_{\aleph_{0}}$, we have to enlarge the above defined set $S(E)$ and decrease $D(E)$. Arguing as in the paragraph for $U_{1}$ (that is, representing $\mathcal{C}(E)$ as $L^{\infty}(\mu)$ for some probabilistic measure $\mu$ ), we see that there is $S_{2} \in \mathcal{P}_{\omega}(S)$ such that $\left.v\right|_{E}=\bigvee_{s \in S_{2}} f_{s}$. By Lemma 15.1, there is an open dense subset $D_{2}$ of $E$ such that $v(x)=\sup _{s \in S_{2}} f_{s}(x)$. Now it suffices to replace $S(E)$ by $S(E) \cup S_{2}$ and $D(E)$ by $D(E) \cap D_{2}$. (The details are left for the reader.)

Both points of Lemma 15.7 yield

15.8. Corollary. Let $\left\{f_{s}\right\}_{s \in S}$ be a nonempty subset of $\Lambda(\Omega)$.

(A) There is an open dense subset $D$ of $\Omega$ such that for all $x \in D$,

$$
\left(\bigwedge_{s \in S} f_{s}\right)(x)=\inf _{s \in S} f_{s}(x)
$$


(B) If $E$ is a clopen subset of $\Omega$ such that $\left(\bigvee_{s \in S} f_{s}\right)(E) \subset I_{\aleph_{0}}$, then there exists an open dense subset $G$ of $E$ such that for any $x \in G$, $\left(\bigvee_{s \in S} f_{s}\right)(x)=\sup _{s \in S} f_{s}(x)$.

15.9. Remark. We suspect that the counterpart of point (A) of Corollary 15.8 for suprema fails to be true in general. However, partial results in this direction may be shown. Let $u=\bigvee_{s \in S} f_{s}$. Put $U_{1}=u^{-1}\left(I_{\aleph_{0}}\right)$ and $U_{\alpha}=\operatorname{int} f^{-1}(\{\alpha\})$ for $\alpha \in \operatorname{Card}_{\infty} \backslash\left\{\aleph_{0}\right\}$. The argument used in the proof of Lemma 15.1 (page 44) shows that $U_{1} \cup \bigcup_{\alpha>\aleph_{0}} U_{\alpha}$ is dense in $\Omega$. By Corollary 15.8, there is an open dense subset of $U_{1}$ such that

$$
u(x)=\sup _{s \in S} f_{s}(x)
$$

for $x \in D_{1}$. We ask for which $\alpha \in \operatorname{Card}_{\infty} \backslash\left\{\aleph_{0}\right\}$ there is an open dense subset $D_{\alpha}$ of $U_{\alpha}$ such that (15-15) is satisfied for all $x \in D_{\alpha}$. It is quite easy to show that this is true when $\alpha=\beta^{+}$for some $\beta \geqslant \aleph_{0}$ (indeed, it suffices to put $D_{\alpha}=U_{\alpha} \cap \bigcup_{s \in S} f_{s}^{-1}(\{\alpha\})$; since $f_{s} \leqslant \alpha$ on $U_{\alpha}$ and $\alpha$ is an isolated point of $I_{\alpha}$, the set $D_{\alpha}$ is open; that $\mathrm{cl} D_{\alpha}=U_{\alpha}$ may be proved by a standard argument on the difference of the latter sets). A little bit more difficult is to prove that $D_{\alpha}$ exists for every limit cardinal $\alpha$ which has countable cofinality. The latter means that there is a sequence $\left(\beta_{n}\right)_{n=1}^{\infty}$ of cardinals such that $\beta_{n}<\alpha$ for every $n$, and $\alpha=\sup _{n \geqslant 1} \beta_{n}$. In that case we put $G=U_{\alpha} \cap \bigcap_{n=1}^{\infty} \bigcup_{s \in S} f_{s}^{-1}\left(\right.$ Card $\left.\backslash I_{\beta_{n}}\right)$ and $D=U_{\alpha} \backslash \operatorname{cl} G$. Our first claim is that $D$ is empty. For if not, there would exist a nonempty clopen set $E \subset D$. Then put $E_{n}=E \cap \bigcap_{s \in S} f_{s}^{-1}\left(I_{\beta_{n}}\right)$. Noticing that $E=\bigcup_{n=1}^{\infty} E_{n}$ (since $E \cap G=\varnothing$ ) and $E_{n}$ 's are closed, infer from Baire's theorem that $W=\operatorname{int} E_{n}$ is nonempty for some $n$ and thus $\bigvee_{s \in S}\left(\left.f_{s}\right|_{W}\right) \leqslant \beta_{n}$ (W is clopen), contradictory to the fact that $\left[\bigvee_{s \in S}\left(\left.f_{s}\right|_{W}\right)\right](x)=u(x)=\alpha$ for $x \in W$. So, $D$ is indeed empty and hence $G$ is a dense $\mathcal{G}_{\delta}$ subset of $U_{\alpha}$. Now an application of Lemma 14.2 (page 40) yields that $D_{\alpha}=\operatorname{int} G$ is dense in $U_{\alpha}$ as well.

The above arguments show that if $\left(\bigvee_{s \in S} f_{s}\right)(\Omega) \cap \operatorname{Card}_{\infty}$ consists only of cardinals which are non-limit or have countable cofinality, then $\bigvee_{s \in S} f_{s}$ may be computed pointwisely on an open dense set.

15.10. Theorem. For every nonempty set $\left\{\mathbb{A}^{(s)}\right\}_{s \in S} \subset \mathcal{C D D} \mathcal{D}_{N}$ and each $\mathbb{B} \in \mathcal{C D D}_{N}$

$$
\begin{aligned}
& \mathbb{B} \wedge\left(\bigvee_{s \in S} \mathbb{A}^{(s)}\right)=\bigvee_{s \in S}\left(\mathbb{B} \wedge \mathbb{A}^{(s)}\right) \\
& \mathbb{B} \vee\left(\bigwedge_{s \in S} \mathbb{A}^{(s)}\right)=\bigwedge_{s \in S}\left(\mathbb{B} \vee \mathbb{A}^{(s)}\right)
\end{aligned}
$$

Proof. As usual, we pass to $\Lambda(\Omega)$. Put $f_{s}=\widehat{\mathbb{A}^{(s)}}$ and $g=\widehat{\mathbb{B}}$. Let $u=\bigwedge_{s \in S} f_{s}$ and $u^{\prime}=\bigwedge_{s \in S}\left(g \vee f_{s}\right)$. By Corollary 15.8, there are open dense sets $D$ and $D^{\prime}$ such that $u(x)=\inf _{s \in S} f_{s}(x)$ for $x \in D$ and 


$$
\begin{aligned}
& u^{\prime}(x)=\inf _{s \in S}\left(g \vee f_{s}\right)(x) \text { for } x \in D^{\prime} . \text { Then for } x \in D \cap D^{\prime}, \\
& \quad(g \vee u)(x)=\max \left(g(x), \inf _{s \in S} f_{s}(x)\right)=\inf _{s \in S}\left(\max \left(g(x), f_{s}(x)\right)=u^{\prime}(x)\right.
\end{aligned}
$$

which gives (15-17). Now we pass to (15-16).

Let $v=\bigvee_{s \in S} f_{s}$ and $v^{\prime}=\bigvee_{s \in S}\left(g \wedge f_{s}\right)$. We only need to show that $v^{\prime} \geqslant g \wedge v$. As usual, put $U_{0}=g^{-1}\left(I_{\aleph_{0}}\right) \cap v^{-1}\left(I_{\aleph_{0}}\right), U_{1}=g^{-1}\left(I_{\aleph_{0}}\right) \backslash$ $v^{-1}\left(I_{\aleph_{0}}\right)$ and $U_{\alpha}=\operatorname{int} g^{-1}(\{\alpha\})$ for $\alpha \in \operatorname{Card}_{\infty} \backslash\left\{\aleph_{0}\right\}$. We know that each of the just defined sets is clopen and their union is dense in $\Omega$. Hence it suffices to show that $g \wedge v \leqslant v^{\prime}$ on a dense subset of $U_{\alpha}$ for any $\alpha \in\{0,1\} \cup \operatorname{Card}_{\infty} \backslash\left\{\aleph_{0}\right\}$.

On $U_{0}$ it suffices to apply Corollary 15.8: if $v^{\prime}(x)=\sup _{s \in S}\left(g \wedge f_{s}\right)(x)$ for $x \in D^{\prime}$ and $v(x)=\sup _{s \in S} f_{s}(x)$ for $x \in D$, then $v^{\prime}=v \wedge g$ on $D \cap D^{\prime}$. Further, since $v>\aleph_{0}$ on $U_{1}$, the set $D_{1}=U_{1} \cap \bigcup_{s \in S} f_{s}^{-1}\left(\right.$ Card $\left.\backslash I_{\aleph_{0}}\right)$ is dense in $U_{1}$. What is more, for every $x \in D_{1}$ there is $s \in S$ with $f_{s}(x)>\aleph_{0}$ and therefore $v^{\prime}(x) \geqslant\left(f_{s} \wedge g\right)(x)=g(x)$. Consequently, $v^{\prime} \geqslant g \wedge v$ on $D_{1}$ and we are done.

Now fix $\alpha \in \operatorname{Card}_{\infty} \backslash\left\{\aleph_{0}\right\}$. We divide $U_{\alpha}$ into two clopen parts: $V_{1}=$ $U_{\alpha} \cap v^{-1}\left(I_{\alpha}\right)$ and $V_{2}=U_{\alpha} \backslash v^{-1}\left(I_{\alpha}\right)$. Let $D_{\alpha}=V_{1} \cup \bigcup_{s \in S}\left[U_{\alpha} \backslash f_{s}^{-1}\left(I_{\alpha}\right)\right]$. Notice that $f_{s} \leqslant \alpha$ on $V_{1}$ (hence $v^{\prime}=v$ on $V_{1}$ ) and for every $x \in D_{\alpha} \backslash V_{1}$ there is $s \in S$ such that $f_{s}(x)>\alpha$ (so, $v^{\prime}=g$ on $D_{\alpha} \backslash V_{1}$ ). This proves that $v^{\prime} \geqslant v \wedge g$ on $D_{\alpha}$. Finally, standard argument shows that $D_{\alpha} \cap V_{2}$ is dense in $V_{2}$ and this finishes the proof.

15.11. Proposition. The assertion of (AO14) (page 31) is satisfied.

Proof. Again, it suffices to prove the counterpart of (AO14) in the realm $\Lambda(\Omega)$. It is clear that $\alpha \cdot(f \vee g)=(\alpha \cdot f) \vee(\alpha \cdot g)$ and $\alpha \cdot(f \wedge g)=$ $(\alpha \cdot f) \wedge(\alpha \cdot g)$ for all $f, g \in \Lambda(\Omega)$ and each $\alpha \in$ Card. Now let $\alpha=k$ be a positive finite cardinal. In order to show that $k \cdot\left(\bigvee_{s \in S} f_{s}\right)=\bigvee_{s \in S}\left(k \cdot f_{s}\right)$ and $k \cdot\left(\bigwedge_{s \in S} f_{s}\right)=\bigwedge_{s \in S}\left(k \cdot f_{s}\right)$, let us consider an 'extended' version of $\Lambda(\Omega)$, namely $\widetilde{\Lambda}(\Omega)$ which is defined in the same way as $\Lambda(\Omega)$ with the only difference that members of $\widetilde{\Lambda}(\Omega)$ send $\Omega_{I}$ into $\mathbb{R}_{+} \cup$ Card. We shall prove in Corollary 23.2 (page 108) that $\Omega_{I}$ is homeomorphic to $\Omega_{I I}$. Consequently, $\widetilde{\Lambda}(\Omega)$ is order-complete. It is immediate that the assignment $\widetilde{\Lambda}(\Omega) \ni f \mapsto k \cdot f \in \widetilde{\Lambda}(\Omega)$ is a bijective order isomorphism. Hence it preserves g.l.b.'s and l.u.b.'s computed in the space $\widetilde{\Lambda}(\Omega)$. So, we only need to check that $u:=\sup _{\widetilde{\Lambda}(\Omega)} F$ and $v:=\inf _{\widetilde{\Lambda}(\Omega)} F$ are members of $\Lambda(\Omega)$ for every nonempty set $F \subset \Lambda(\Omega)$. Since the proof for $u$ is similar, we shall only show that $v \in \Lambda(\Omega)$. Let $D_{0}=$ $\Omega_{I} \cap \operatorname{int} v^{-1}(\{0\}), B_{0}=v^{-1}(\{0\}) \cap \Omega_{I} \backslash D_{0}$ and for positive integer $m$ let $D_{m}=\Omega_{I} \cap \operatorname{int} v^{-1}((m-1, m])$ and $B_{m}=v^{-1}((m-1, m]) \cap \Omega_{I} \backslash D_{m}$. We claim that $D=\left(\Omega_{I} \cap v^{-1}\left(\operatorname{Card}_{\infty}\right)\right) \cup \bigcup_{m=0}^{\infty} D_{m}$ is dense in $\Omega_{I}(D$ is of course open). Indeed, $\Omega_{I} \backslash D=\bigcup_{m=0}^{\infty} B_{m}$. Since each of $B_{m}$ 's is nowhere dense (by Lemma 14.2, page 40), Baire's theorem yields our assertion. Now let $v^{\prime} \in \Lambda(\Omega)$ be a function such that $v^{\prime}=v$ on 
$\left(\Omega_{I} \cap v^{-1}\left(\operatorname{Card}_{\infty}\right)\right) \cup \Omega_{I I} \cup \Omega_{I I I}$ and $v\left(D_{m}\right) \subset\{m\}$ for every integer $m \geqslant 0$ (see Lemma 14.1, page 40). We see that $v(x) \leqslant v^{\prime}(x)$ for $x \in$ $D \cup \Omega_{I I} \cup \Omega_{I I I}$ and consequently $v \leqslant v^{\prime}$. Moreover, since $v \leqslant f \in \Lambda(\Omega)$ for any $f \in F, v^{\prime} \leqslant f$ as well $(f \in F)$ and hence $v=v^{\prime} \in \Lambda(\Omega)$.

In the second part of the second claim of (AO14) one assumes that $\mathbb{E}_{s m}\left(\mathbb{A}^{(s)}\right)=\mathbb{O}$ which corresponds to $f_{s}\left(\Omega_{I I}\right) \subset\{0\} \cup$ Card $_{\infty}$. Here we shall weaken this, assuming that $f_{s}\left(\Omega_{I I}\right) \subset$ Card for each $s \in S$. It follows from Corollary 15.8 that there is an open dense subset $D$ of $\Omega$ such that for all $x \in D,\left(\bigwedge_{s \in S} f_{s}\right)(x)=\inf _{s \in S} f_{s}(x)$ as well as $\left[\left(\bigwedge_{s \in S}\left(\alpha \cdot f_{s}\right)\right](x)=\inf _{s \in S}\left(\alpha \cdot f_{s}\right)(x)\right.$. Since all values of (all) $f_{s}$ 's are cardinals, we see that in both the latter formulas 'inf' may be replaced by 'min'. But $\alpha \cdot \min _{s \in S} f_{s}(x)=\min _{s \in S}\left(\alpha \cdot f_{s}(x)\right)$ and thus $\alpha \cdot\left(\bigwedge_{s \in S} f_{s}\right)(x)=\left[\bigwedge_{s \in S}\left(\alpha \cdot f_{s}\right)\right](x)$ for $x \in D$ and we are done.

We now pass to the last claim: that $\alpha \cdot \bigvee_{f \in F} f=\bigvee_{f \in F}(\alpha \cdot f)$ for every nonempty set $F \subset \Lambda(\Omega)$ and $\alpha \in \operatorname{Card}_{\infty}$. The inequality ' $\geqslant$ ' is clear. To prove the converse, put $u=\bigvee_{f \in F}(\alpha \cdot f)$. It is enough to show that $\alpha \cdot u=u$. Equivalently, we have to check that for each $x \in \Omega, u(x) \geqslant \alpha$ or $u(x)=0$. Suppose, for the contrary, that $0<$ $u\left(x_{0}\right)<\alpha$ for some $x_{0} \in \Omega$. Take a closed set $B \subset I_{\alpha} \backslash\{\alpha\}$ such that $u\left(x_{0}\right) \in \operatorname{int} B$ and put $D=\operatorname{int} u^{-1}(B) . D$ is clopen and $x_{0} \in D$. Now let $u^{\prime} \in \Lambda(\Omega)$ be given by $u^{\prime}=u$ on $\Omega \backslash D$ and $u^{\prime}=0$ on $D$. We see that $u^{\prime}\left(x_{0}\right)<u\left(x_{0}\right)$. However, $\alpha \cdot f \leqslant u^{\prime}$ for every $f \in F$. Indeed, if $x \in D$, then $\alpha>u(x) \geqslant \alpha \cdot f(x)$ which implies that $f(x)=0$. Thus, $u$ is not the l.u.b. of $\alpha \cdot F$ and this finishes the proof.

15.12. Remark. It is natural to ask which element of $\Lambda(\Omega)$ corresponds to $\mathbb{A}=\bigoplus_{s \in S} \mathbb{A}^{(s)}$ for an uncountable set $S$. In other words, how to express $\sum_{s \in S} f_{s}:=\widehat{\mathbb{A}}$ by means of $f_{s}=\widehat{\mathbb{A}^{(s)}}(s \in S)$. Lemma 15.1 (page 44) and Theorem 15.2 (page 46) show that for countable $S$, $\sum_{s \in S} f_{s}$ may be computed pointwisely on an open dense subset of $\Omega$. Let us demonstrate how to find $\sum_{s \in S} f_{s}$ when $S$ is uncountable. We shall use here the arguments of Section 13. First of all, let $g=$ $\bigvee\left\{\sum_{s \in S^{\prime}} f_{s}: \quad S^{\prime} \in \mathcal{P}_{f}(S)\right\}$ and $U_{f}=\operatorname{cl} g^{-1}\left(\mathbb{R}_{+}\right)$. It may be deduced from the arguments of Section 13 that $\sum_{s \in S} f_{s}=g$ on $U_{f}$ and the function $f:=\sum_{s \in S} f_{s}$ takes infinite values on $\Omega \backslash U_{f}$. So, we only need to characterize $U_{\alpha}=\operatorname{int} f^{-1}(\{\alpha\})$ for $\alpha \in \operatorname{Card}_{\infty}$ (since we know that $U_{f} \cup \bigcup_{\alpha \in \operatorname{Card}_{\infty}} U_{\alpha}$ is dense in $\Omega$ ). This is possible thanks to (13-1) (page 35). For this purpose, we define $\operatorname{dim}_{E} u$ for $u \in \Lambda(\Omega)$ and a nonempty clopen set $E \subset \Omega$ with $c(E)=\aleph_{0}$ as follows:

$$
\begin{aligned}
\operatorname{dim}_{E} u=\sum\left\{\alpha \in \operatorname{Card}_{\infty}: E \cap \operatorname{int} u^{-1}\right. & (\{\alpha\}) \neq \varnothing\} \\
& +c_{*}\left(E \cap \operatorname{cl} u^{-1}\left(\mathbb{R}_{+} \backslash\{0\}\right)\right)
\end{aligned}
$$

(notice that the latter summand is either 0 or $\aleph_{0}$ ). Now one may conclude from (13-1) that $U_{\alpha}$ is the closure of the union of all clopen 
sets $V \subset \Omega \backslash U_{f}$ such that $\sum_{s \in S} \operatorname{dim}_{E} f_{s}=\alpha$ for every nonempty clopen set $E \subset V$ with $c(E)=\aleph_{0}$ (of course, $U_{\alpha}$ may be empty). We leave the details for the interested reader.

15.13. Remark. It is clear that the formula for $\Phi_{\mathbb{T}}$ essentially depends on $\mathbb{T}$. However, there is a quite simple connection between $\Phi_{\mathbb{T}}$ and $\Phi_{\mathbb{S}}$ for any two semiminimal $N$-tuples $\mathbb{T}$ and $\mathbb{S}$ such that $\aleph_{0} \odot \mathbb{T}=\aleph_{0} \odot \mathbb{S}=\mathbb{J}_{I I}$. Put $u=j_{\Omega_{I} \cup \Omega_{I I I}}+\frac{\mathrm{d} \mathbb{S}}{\mathrm{dT}}$ and $D:=u^{-1}\left(\mathbb{R}_{+} \backslash\{0\}\right)$. We leave this as an easy exercise that $D$ is dense in $\Omega$ and for every $\mathbb{X} \in \mathcal{C D} \mathcal{D}_{N}, \Phi_{\mathbb{S}}(\mathbb{X})$ is the unique continuous extension of $\left.\left(\frac{1}{u} \Phi_{\mathbb{T}}(\mathbb{X})\right)\right|_{D}$.

\section{TYPES OF $N$-TUPLES}

As in the previous section, $\widehat{\mathbb{A}}=\Phi_{\mathbb{T}}(\mathbb{A})$ for each $\mathbb{A} \in \mathcal{C D} \mathcal{D}_{N}$ where $\Phi_{\mathbb{T}}$ is as in Theorem 15.2 (page 46). This notation is obligatory to the end of the paper.

The following result is an immediate consequence of Proposition 10.1 (page 25).

16.1. Proposition. For every clopen set $E \subset \Omega$ the class $\mathcal{J}[E]:=\{\mathbb{A} \in$ $\left.\mathcal{C D D}_{N}: \operatorname{supp} \widehat{\mathbb{A}} \subset E\right\}$ is an ideal in $\mathcal{C D D}_{N}$. Conversely, for every ideal $\mathcal{A} \subset \mathcal{C D D}_{N}$ there is a (unique) clopen set $K \subset \Omega$ such that $\mathcal{A}=\mathfrak{J}[K]$. What is more, $K=\operatorname{supp} \widehat{\mathbb{J ( A})}$.

For every ideal $\mathcal{A}$, the unique clopen set $K$ such that $\mathcal{A}=\mathcal{J}[K]$ will be denoted by $\operatorname{supp}_{\Omega} \mathcal{A}$. Below we give some illustrative examples related to this subject.

16.2. Examples. (A) Fix nonnegative real number $r$ and let $\mathcal{J}(r)$ be the class of all $N$-tuples $\mathbb{X}$ for which $\|\mathbb{X}\| \leqslant r$. It is clear that $\mathcal{J}(r)$ is an ideal. Put $\Omega(r):=\operatorname{supp}_{\Omega} \mathcal{J}(r)$ and $\Omega(\mathrm{bd}):=\bigcup_{r \geqslant 0} \Omega(r)$. The set $\Omega(\mathrm{bd})$ is open in $\Omega$ and for every $\mathbb{X} \in \mathcal{C D} \mathcal{D}_{N}$,

$$
\|\mathbb{X}\|<\infty \Longleftrightarrow \operatorname{supp} \widehat{\mathbb{X}} \subset \Omega(\mathrm{bd})
$$

(indeed, use the fact that supp $\widehat{\mathbb{X}}$ is compact). What is more, if $\|\mathbb{X}\|<\infty$, then $\|\mathbb{X}\|=\min \{r \geqslant 0: \operatorname{supp} \widehat{\mathbb{X}} \subset \Omega(r)\}$. The ideal $\mathcal{J}[\mathrm{cl} \Omega(\mathrm{bd})]$ consists of all $N$-tuples which are direct sums of bounded $N$-tuples. Further, whenever $0 \leqslant r<s$, the ideal $\mathcal{J}[\Omega(r) \backslash$ $\Omega(s)$ ] consists of all $N$-tuples whose every nontrivial reduced part has norm greater than $s$ but no greater than $r$. We conclude from this that $\Omega(s)=\operatorname{int}\left(\bigcap_{r>s} \Omega(r)\right)$ for any $s \geqslant 0$. For positive $r$ put $\Omega\{r\}=\Omega(r) \backslash \operatorname{cl}\left(\bigcup_{s<r} \Omega(s)\right)$ and $\mathcal{J}\{r\}=\mathcal{J}[\Omega\{r\}]$. The ideal $\mathcal{J}\{r\}$ constists of all $N$-tuples whose every nontrivial reduced part has norm equal to $r$.

(B) Now let $\mathcal{J}(\mathfrak{b}):=\left\{\mathfrak{b}(\mathbb{A}): \mathbb{A} \in \mathcal{C D} \mathcal{D}_{N}\right\}$. It follows from suitable properties of the $\mathfrak{b}$-transform that $\mathcal{J}(\mathfrak{b})$ is an ideal. Let $\Omega(\mathfrak{b})=$ $\operatorname{supp}_{\Omega} \mathcal{J}(\mathfrak{b})$. Notice that $\mathcal{J}(\mathfrak{b})$ consists of all $N$-tuples $\mathbb{X}$ such that 
either $\|\mathbb{X}\|<1$ or $\|\mathbb{X}\|=1$ and $\mathbb{X}$ does not assume its norm. Consequently, $\Omega(\mathfrak{b}) \varsubsetneqq \Omega(1)$. The ideal $\mathcal{J}[E]$ with $E=\Omega(1) \backslash \Omega(\mathfrak{b})$ constists of all $N$-tuples whose each nontrivial reduced part has norm 1 and assumes its norm. In particular, $E \subset \Omega\{1\}$ and the ideal $\mathcal{J}[\Omega\{1\} \backslash E]=\mathcal{J}[\Omega\{1\} \cap \Omega(\mathfrak{b})]$ coincides with the class of all $N$-tuples whose every nontrivial reduced part has norm equal to 1 and does not assume its norm.

As a consequence of Theorem 5.1 (page 10) and Examples 16.2 we obtain

16.3. Corollary. Every contraction $T$ acting on a Hilbert space $\mathcal{H}$ induces a unique decomposition $\mathcal{H}=\mathcal{H}_{0} \oplus \mathcal{H}_{1} \oplus \mathcal{H}_{2}$ such that $\mathcal{H}_{0}, \mathcal{H}_{1}, \mathcal{H}_{2} \in$ $\operatorname{red}(T)$ and

(a) every nontrivial reduced part of $\left.T\right|_{\mathcal{H}_{0}}$ admits a nontrivial reduced part of norm less than 1 ,

(b) $\left.T\right|_{\mathcal{H}_{1}}$ does not assume its norm (unless $\mathcal{H}_{1}$ is trivial) and every its nontrivial reduced part has norm equal to 1 ,

(c) every nontrivial reduced part of $\left.T\right|_{\mathcal{H}_{2}}$ has norm 1 and assumes its norm.

What is more, $\mathcal{H}_{0}, \mathcal{H}_{1}, \mathcal{H}_{2} \in \operatorname{cred}(T)$.

As it was done by Ernest [9], the types of $\mathcal{W}^{\prime \prime}(X)$ and $\mathcal{W}^{\prime}(X)$ may be assigned to $X$. It is easily seen (and in fact, this was used by us in Theorem 11.1, page 26) that for every nontrivial $X \in \mathrm{CDD}_{N}$ :

- $\mathcal{W}^{\prime}(\boldsymbol{X})$ is type $\mathrm{I}_{\alpha}(\alpha \in$ Card $\backslash\{0\})$ iff $\mathbb{X}=\alpha \odot \mathbb{E}$ for some unique $\mathbb{E} \leqslant s \mathbb{J}_{I}$,

- $\mathcal{W}^{\prime}(X)$ is type $\operatorname{III}_{\alpha}\left(\alpha \in \operatorname{Card}_{\infty}\right)$ iff $\mathbb{X}=\alpha \odot \mathbb{E}$ for some unique $\mathbb{E} \leqslant \mathbb{J}_{I I I}$,

- $\mathcal{W}^{\prime}(X)$ is type $\mathrm{II}_{1}$ iff $\mathbb{X}$ is semiminimal,

- $\mathcal{W}^{\prime}(X)$ is type $\operatorname{II}_{\alpha}\left(\alpha \in \operatorname{Card}_{\infty}\right)$ iff $\mathbb{X}=\alpha \odot \mathbb{E}$ for some unique $\mathbb{E} \leqslant \mathbb{J}_{I I}$.

Ernest calls a bounded operator $T$ of type $i_{\alpha}$ provided $\mathcal{W}^{\prime}(T)$ is of this type (cf. [9, Definition 1.28]). We call a nontrivial $N$-tuple $\mathbb{X} \in \mathcal{C D} \mathcal{D}_{N}$ (of) type $\mathrm{I}^{n}$ (with $\left.n=1,2, \ldots, \infty\right)$ ) $\mathrm{II}^{1}$, $\mathrm{II}^{\infty}$ or $\mathrm{III}^{(\infty)}$ iff $\mathcal{W}^{\prime \prime}(X)$ is of this type. Additionally, we agree that the trivial $N$-tuple is of each of these types.

Since a von Neumann algebra is type I, II, III iff so is its commutant, we see that for nontrivial $\mathbb{X}, \mathcal{W}^{\prime \prime}(X)$ is type III iff so is $\mathcal{W}^{\prime}(X)$ and thus the above definition makes no confusion. Later we shall see that if nontrivial $\mathbb{X}$ is type $i^{\infty}(i \in\{I, I I, I I I\})$, then $\mathcal{W}^{\prime \prime}(X)$ is type $i_{\aleph_{0}}$ and thus there is no need to use uncountable cardinals here.

Fix $i^{n} \in\left\{I^{1}, I^{2}, \ldots, I^{\infty}, I I^{1}, I I^{\infty}, I I I^{\infty}\right\}$ and let $\mathcal{J}_{i_{n}}$ be the class of all $N$-tuples of type $i^{n}$. Our first goal is

16.4. Proposition. $\mathcal{J}_{i_{n}}$ is an ideal in $\mathrm{eDD}_{N}$. 
Proof. It suffices to verify all points of Corollary 11.6 (page 27). The point (a) is fulfilled since for every $\alpha \in \operatorname{Card}_{\infty}$ and nontrivial $\mathbb{X}$, the von Neumann algebras $\mathcal{W}^{\prime \prime}(\boldsymbol{X})$ and $\mathcal{W}^{\prime \prime}(\boldsymbol{Y})$ are isomorphic where $\mathbb{Y}=\alpha \odot \mathbb{X}$. Point (b) follows from the following result on $\mathcal{W}^{*}$-algebras: if $\mathcal{M}$ is a $\mathcal{W}^{*}$-algebra and $\left\{z_{s}\right\}_{s \in S}$ is a family of mutually orthogonal central projections in $\mathcal{M}$ which sum up to 1 and $\mathcal{M} z_{s}$ is type $i_{n}$ for each $s \in S$, then $\mathcal{M}$ itself is type $i_{n}$. Finally, point (c) is a consequence of a similar result: if $\mathcal{M}$ is a type $i_{n} \mathcal{W}^{*}$-algebra and $z$ is a (nonzero) central projection in $\mathcal{M}$, then $\mathcal{M} z$ is type $i_{n}$ as well.

Now put $\Omega_{i_{n}}=\operatorname{supp}_{\Omega} \mathcal{J}_{i_{n}}$. It is clear that the sets $\Omega_{I_{1}}, \Omega_{I_{2}}, \ldots, \Omega_{I_{\infty}}$, $\Omega_{I I_{1}}, \Omega_{I I_{\infty}}$ and $\Omega_{I I I_{\infty}}$ are pairwise disjoint and their union is dense in $\Omega$. It is obvious that $\Omega_{I I I_{\infty}}=\Omega_{I I I}$. Let us now check that if $\mathbb{X}$ is type $i^{\infty}$ (and nontrivial), then $\mathcal{W}^{\prime \prime}(\boldsymbol{X})$ is type $i_{\aleph_{0}}$. Indeed, there is $\mathbb{E} \leqslant^{s} \mathbb{J}$ (namely, $\mathbb{E}=s(\mathbb{X})$, cf. (15-5), page 46) and an infinite cardinal $\alpha$ such that $\alpha \odot \mathbb{X}=\alpha \odot \mathbb{E}$. The latter implies that $\mathcal{W}^{\prime \prime}(\boldsymbol{X})$ and $\mathcal{W}^{\prime \prime}(\boldsymbol{E})$ are isomorphic as $\mathcal{W}^{*}$-algebras and thus $\mathcal{W}^{\prime \prime}(\boldsymbol{E})$ is type $i_{\infty}$. Further, we conclude from Proposition 9.10 (page 24) that $\mathbb{E}=\boxplus_{s \in S} \mathbb{E}^{(s)}$ for suitable family such that $0<\operatorname{dim}\left(\mathbb{E}^{(s)}\right) \leqslant \aleph_{0}$. Consequently, $\mathcal{W}^{\prime \prime}\left(\boldsymbol{E}^{(s)}\right)$ is type $i_{\infty}$ for each $s \in S$ and therefore (since $\boldsymbol{E}^{(s)}$ act in a separable Hilbert space) $\mathcal{W}^{\prime \prime}\left(\boldsymbol{E}^{(s)}\right)$ is type $i_{\aleph_{0}}$. This yields that $\mathcal{W}^{\prime \prime}(\boldsymbol{E})$ (and hence $\left.\mathcal{W}^{\prime \prime}(X)\right)$ is type $i_{\aleph_{0}}$ as well.

One may easily check that $\mathcal{J}_{I_{1}}$ coincides with the ideal $\mathcal{N}_{N}$ introduced in Examples 5.3-(E) (page 12) and studied in Example 10.3 (page 25). Thus $\Omega_{I_{1}}$ corresponds to normal $N$-tuples.

The sets $\Omega_{I_{n}}$ may be used to compute $\operatorname{dim}(\mathbb{X})$ for every $\mathbb{X} \in \mathcal{C D} \mathcal{D}_{N}$ by means of $\widehat{\mathbb{X}}$. For this, let us introduce the strict Souslin number, $c_{f}(X)$, of a topological space $X$. Namely, $c_{f}(X)=c(X)$ iff $X$ is an infinite set and $c_{f}(X)=\operatorname{card}(X)$ otherwise.

16.5. Proposition. Let $\mathbb{X} \in \mathcal{C D D}_{N}, f=\widehat{\mathbb{X}}, U_{\alpha}^{i}=\Omega_{i} \cap \operatorname{int} f^{-1}(\{\alpha\})$ for $(i, \alpha) \in \Upsilon_{*}$ and $U_{1}^{I I}=\Omega_{I I} \cap \mathrm{cl} f^{-1}\left(\mathbb{R}_{+} \backslash\{0\}\right)$. Then

$$
\begin{aligned}
& \operatorname{dim}(\mathbb{X})=\sum_{n, m=1}^{\infty} n m \cdot c_{f}\left(U_{n}^{I} \cap \Omega_{I_{m}}\right)+\aleph_{0} \sum_{n=1}^{\infty} c_{f}\left(U_{n}^{I} \cap \Omega_{I_{\infty}}\right) \\
&+\aleph_{0} \cdot c_{f}\left(U_{1}^{I I}\right)+\sum_{\alpha \in \operatorname{Card}_{\infty}} \alpha\left[c_{f}\left(U_{\alpha}^{I}\right)+c_{f}\left(U_{\alpha}^{I I}\right)+c_{f}\left(U_{\alpha}^{I I I}\right)\right] .
\end{aligned}
$$

Proof. As in the proof of Proposition 15.5 (page 50), we see that $\operatorname{dim}(\mathbb{X})=\sum_{(i, \alpha) \in \Upsilon} \alpha \cdot \operatorname{dim}\left(\mathbb{E}_{\alpha}^{i}(\mathbb{X})\right)$ and $\aleph_{0} \cdot \operatorname{dim}\left(\mathbb{E}_{\alpha}^{i}(\mathbb{X})\right)=c_{*}\left(U_{\alpha}^{i}\right)=$ $\aleph_{0} \cdot c_{f}\left(U_{\alpha}^{i}\right)$. Moreover, $\operatorname{dim}\left(\mathbb{E}_{1}^{I I}(\mathbb{X})\right) \in \operatorname{Card}_{\infty} \cup\{0\}$. So, to show (16-1), it suffices to check that $\operatorname{dim}\left(\mathbb{E}_{n}^{I}(\mathbb{X})\right)=\aleph_{0} \cdot c_{f}\left(U_{n}^{I} \cap \Omega_{I_{\infty}}\right)+\sum_{m=1}^{\infty} m$. $c_{f}\left(U_{n}^{I} \cap \Omega_{I_{m}}\right)$. Write $\mathbb{E}_{n}^{I}(\mathbb{X})=\bigoplus_{m=1}^{m=\infty} \mathbb{E}_{n, m}$ with $\mathbb{E}_{n, m} \in \mathcal{J}_{I_{m}}$ and observe that $\operatorname{supp}_{\Omega} \mathbb{E}_{n, m}=U_{n}^{I} \cap \Omega_{I_{m}}=: V_{n, m}$. So, it is enough to show that

$$
\operatorname{dim}\left(\mathbb{E}_{n, m}\right)=m \cdot c_{f}\left(V_{n, m}\right)
$$


(for $m=\infty$ the above means that $\operatorname{dim}\left(\mathbb{E}_{n, \infty}\right)=\aleph_{0} \cdot c_{f}\left(V_{n, \infty}\right)$ ). If the set $V_{n, m}$ is infinite, then we may 'divide' it into arbitrarily (finitely) many pairwise disjoint nonempty clopen sets which yields that representatives of $\mathbb{E}_{n, m}$ act in infinite-dimensional Hilbert spaces and hence (16-2) is fulfilled in that case (e.g. by Proposition 15.5). On the other hand, if $V_{n, m}$ is finite, $\mathbb{E}_{n, m}$ may be decomposed into card $\left(V_{n, m}\right)$ irreducible $N$-tuples of type $I^{m}$. Now (16-2) easily follows since an irreducible $N$-tuple of type $I^{m}$ acts in an $m$-dimensional Hilbert space.

\section{Primes, Semiprimes, atoms and fractals}

Prime numbers may be defined in two ways (below $n, k$ and $l$ are positive integers):

- $n$ is prime iff $n \neq 1$, and $n=k l$ implies $k=1$ or $l=1$,

- $n$ is prime iff $n \neq 1$, and $n=k l$ implies $k, l \in\{1, n\}$.

These two conditions may naturally be adapted in more general algebraic structures (especially in monoids, i.e. semigroups with neutral elements). However, in some structures they may be nonequivalent. We will see in the sequel that this occurs in $\mathcal{C D} \mathcal{D}_{N}$. Therefore we distinguish the following two classes of $N$-tuples.

17.1. Definition. Let $\mathbb{A} \in \mathcal{C D} \mathcal{D}_{N}$ be nontrivial. We say $\mathbb{A}$ is a prime iff $\mathbb{A}=\mathbb{X} \oplus \mathbb{Y}$ implies $\mathbb{X}, \mathbb{Y} \in\{\mathbb{O}, \mathbb{A}\}$. $\mathbb{A}$ is an atom iff $\mathbb{A}=\mathbb{X} \oplus \mathbb{Y}$ implies $\mathbb{X}=\mathbb{O}$ or $\mathbb{Y}=\mathbb{O}$.

In case of a single bounded operator, our definition of an atom is equivalent to Ernest's one of an irreducible operator ([9]). It is clear that every atom is a prime. But not conversely. To convince of this, let us first shortly prove

17.2. Proposition. For a nontrivial $\mathbb{A} \in \mathcal{C D D}_{N}$ the following conditions are equivalent:

(i) $\mathcal{W}^{\prime}(A)$ is a factor,

(ii) $\mathcal{W}^{\prime \prime}(A)$ is a factor,

(iii) $\left\{\mathbb{X} \in \mathcal{C D D}_{N}: \mathbb{X} \leqslant s\right\}=\{\mathbb{O}, \mathbb{A}\}$,

(iv) exactly one of the following three conditions is fulfilled:

(a) there are unique $\mathbb{X} \in \mathcal{M F F}_{N}$ and a unique positive cardinal $\alpha$ such that $\mathbb{A}=\alpha \odot \mathbb{X}$ and $\mathcal{W}^{\prime}(\boldsymbol{X})$ constists precisely of scalar multiples of the identity operator; what is more, $0<\operatorname{dim}(\mathbb{X}) \leqslant$ $\aleph_{0}$,

(b) there are unique $\mathbb{X} \in \mathcal{H}_{\mathcal{J M}}$ and a unique infinite cardinal $\alpha$ such that $\mathbb{A}=\alpha \odot \mathbb{X}$ and $\mathcal{W}^{\prime}(\boldsymbol{X})$ is a (type III) factor; what is more, $\operatorname{dim}(\mathbb{X})=\aleph_{0}$,

(c) there are (nonunique) $\mathbb{X} \in \mathcal{S M}_{N}$ and a unique cardinal $\alpha \in$ $\{1\} \cup \operatorname{Card}_{\infty}$ such that $\mathbb{A}=\alpha \odot \mathbb{X}$ and $\mathcal{W}^{\prime}(X)$ is a (type $I I_{1}$ ) factor; what is more, $\operatorname{dim}(\mathbb{X})=\aleph_{0}$. 
Proof. The points (i) and (ii) are clearly equivalent. Further, it follows from (PR3) (page 9) that (i) is equivalent to (iii). Consequently, we infer from Theorem 11.1 (page 26) that if $\mathcal{W}^{\prime}(\boldsymbol{A})$ is a factor, then either $\mathbb{A}=\mathbb{E}_{s m}(\mathbb{A})$ or $\mathbb{A}=\beta \odot \mathbb{E}_{\beta}^{i}(\mathbb{A})$ for some $(i, \beta) \in \Upsilon_{*}$. In the first situation put $\mathbb{X}=\mathbb{E}_{s m}$ and $\alpha=1$; in the second one we distinguish between two cases: if $i \neq I I$, put $\mathbb{X}=\mathbb{E}_{\beta}^{i}(\mathbb{A})$, otherwise take $\mathbb{X} \in \mathcal{S M}_{N}$ such that $\aleph_{0} \odot \mathbb{X}=\mathbb{E}_{\beta}^{I I}$; in both cases we put $\alpha=\beta$. Note that $\mathbb{A}=\alpha \odot \mathbb{X}$. Further, we conclude from (PR6) (page 9) that $\mathcal{W}^{\prime}(\boldsymbol{A})$ is a factor iff so is $\mathcal{W}^{\prime}(\boldsymbol{X})$. Now Proposition 9.10 (page 24) implies that $\operatorname{dim}(\mathbb{X}) \leqslant \aleph_{0}$ provided $\left\{\mathbb{Y} \in \mathcal{C D D}_{N}: \mathbb{Y} \leqslant \leqslant^{s} \mathbb{X}\right\}=\{\mathbb{O}, \mathbb{X}\}$. All these notices show that (i) is equivalent to (iv).

We now have

17.3. Proposition. Let $\mathbb{A} \in \mathcal{C D} \mathcal{D}_{N}$ be nontrivial.

(A) $\mathbb{A}$ is an atom iff $\mathcal{W}^{\prime}(A)$ consists precisely of scalar multiples of the identity operator. If $\mathbb{A}$ is an atom, then $\mathbb{A} \leqslant \mathbb{J}_{I}$ and $0<\operatorname{dim}(\mathbb{A}) \leqslant$ $\aleph_{0}$.

(B) Suppose $\mathbb{A} \in \mathcal{C D} \mathcal{D}_{N}$ is not an atom. Then $\mathbb{A}$ is a prime iff $\operatorname{dim}(\mathbb{A})=\aleph_{0}$ and $\mathcal{W}^{\prime}(\boldsymbol{A})$ is a type III factor.

Proof. Point (A) is left for the reader. We pass to (B).

First note that if $\mathbb{A}$ is type III, then $\mathbb{A} \ll \mathbb{J}_{I I I}$. Consequently, if in addition $\operatorname{dim} \overline{\mathbb{A}}=\aleph_{0}, \mathbb{A}=\mathbb{E}_{\aleph_{0}}^{I I I}(\mathbb{A})$ and thus $\mathbb{A}$ is minimal. But then $\left\{\mathbb{X} \in \mathcal{C D D}_{N}: \mathbb{X} \leqslant \mathbb{A}\right\}=\left\{\mathbb{X} \in \mathcal{C D D}_{N}: \mathbb{X} \leqslant s \mathbb{A}\right\}$. So, the sufficiency of the conditions formulated in proposition for $\mathbb{A}$ to be a prime follows from Proposition 17.2. Conversely, if $\mathbb{A}$ is a prime but not an atom, an application of Proposition 17.2 gives us that $\mathbb{A}=\alpha \odot \mathbb{X}$ for suitable $\alpha$ and $\mathbb{X}$. Since $\mathbb{X} \leqslant \mathbb{A}$, we infer that $\mathbb{A}=\mathbb{X}$. So, $\mathbb{X} \notin \mathcal{M} \mathcal{F}_{N}$ (because $\mathbb{A}$ is not an atom) and $\mathbb{X}$ is not semiminimal since $\mathbb{O} \neq \frac{1}{2} \odot \mathbb{Y} \supsetneqq \mathbb{Y}$ for every nontrivial $\mathbb{Y} \in \mathcal{S M}_{N}$. We infer from this that $\mathbb{X} \in \mathcal{H J M M}_{N}$. Thus, $\mathcal{W}^{\prime}(\boldsymbol{A})$ is type III and, of course, it is a factor.

Let $\mathbb{A}$ be a prime which is not an atom. It follows from Proposition 17.3 that $\mathbb{A}=\aleph_{0} \odot \mathbb{A}$. Consequently, $\operatorname{red}(\boldsymbol{A})$ is an infinite set. However, for every $E \in \operatorname{red}(\boldsymbol{A}),\left.\boldsymbol{A}\right|_{E} \equiv \boldsymbol{A}$ (because $\mathbb{A}$ is prime). Conversely, if $\boldsymbol{B} \in \mathrm{CDD}_{N}$ is such that $\operatorname{card}(\operatorname{red}(\boldsymbol{B}))>2$ and $\left.\boldsymbol{B}\right|_{E} \equiv \boldsymbol{B}$ for any $E \in \operatorname{red}(\boldsymbol{B})$, then $\mathbb{B}$ is a prime and not an atom. This observation leads us to

17.4. Definition. A fractal is a prime which is not an atom.

We see that every prime $\mathbb{A}$ is either an atom (if $\mathbb{A} \neq 2 \odot \mathbb{A}$ ) or a fractal (if $\mathbb{A}=2 \odot \mathbb{A}$ ) and that $\mathbb{A}$ is type I or type III. It is immediate that two different primes are unitarily disjoint.

A counterpart of primes for type II $N$-tuples are semiprimes.

17.5. Definition. A nontrivial $N$-tuple $\mathbb{A}$ is said to be a semiprime iff $\mathbb{A}$ is not of the form $n \odot \mathbb{B}$ where $n$ is a natural number and $\mathbb{B}$ is a 
prime and the following condition is fulfilled: whenever $\mathbb{O} \neq \mathbb{X} \leqslant \mathbb{A}$, there is a natural number $m$ such that $\mathbb{A} \leqslant m \odot \mathbb{X}$.

Semiprimes may be characterized as follows.

17.6. Proposition. (I) A nontrivial $N$-tuple $\mathbb{A}$ is a semiprime iff $\mathcal{W}^{\prime}(A)$ is a type $I I_{1}$ factor.

(II) Let $\mathbb{A}$ be a semiprime. Then $\mathbb{A}$ is semiminimal and $\operatorname{dim}(\mathbb{A})=$ $\aleph_{0}$. If $\mathbb{B} \ll \mathbb{A}$, then $\mathbb{B}$ is a semiprime iff $\mathbb{B}=t \odot \mathbb{A}$ for some $t \in \mathbb{R}_{+} \backslash\{0\}$.

Proof. First assume that $\mathcal{W}^{\prime}(\boldsymbol{A})$ is a type $\mathrm{II}_{1}$ factor. Then necessarily $\mathbb{A} \neq n \odot \mathbb{B}$ for any prime $\mathbb{B}$, and $\mathbb{A} \in \mathcal{S} \mathcal{M}_{N}$. Moreover, $\mathcal{W}^{\prime}\left(\aleph_{0} \odot \boldsymbol{A}\right)$ is a factor as well. We conclude from this that $\operatorname{supp}_{\Omega_{I I}} \mathbb{A}$ consists of a single point (see Section 14). This implies that if $\mathbb{O} \neq \mathbb{X} \leqslant \mathbb{A}$, then $\frac{d \mathbb{X}}{d \mathbb{A}}=\lambda \cdot \frac{d \mathbb{A}}{d \mathbb{A}}$ for some real number $\lambda>0$. But $\lambda \cdot \frac{\mathrm{d} \mathbb{A}}{d \mathbb{A}}=\frac{\mathrm{d}(\lambda \odot \mathbb{A})}{\mathrm{d} \mathbb{A}}$ and therefore $\mathbb{X}=\lambda \odot \mathbb{A}$. Now it suffices to take a natural number $m$ such that $m \lambda \geqslant 1$ to ensure that $\mathbb{A} \leqslant m \odot \mathbb{X}$. Consequently, $\mathbb{A}$ is a semiprime.

We now assume that $\mathbb{A}$ is a semiprime. Observe that then $\mathbb{A}=$ $\mathbb{X} \boxplus \mathbb{Y}$ implies $\mathbb{X}=\mathbb{O}$ or $\mathbb{Y}=\mathbb{O}$. We infer from this that $\mathcal{W}^{\prime}(A)$ is a factor. So, according to Proposition $17.2, \mathbb{A}=\alpha \odot \mathbb{X}$ for suitable $\alpha$ and $\mathbb{X}$. Since $\mathbb{A}$ is a semiprime and $\mathbb{O} \neq \mathbb{X} \leqslant \mathbb{A}, \alpha \odot \mathbb{X} \leqslant m \odot \mathbb{X}$ for some natural number $m$. This implies that either $\mathbb{A}=\mathbb{X} \in \mathcal{H}^{\mathcal{T} \mathcal{M}_{N}}$ or $\alpha \leqslant m$. Again taking into account that $\mathbb{A}$ is a semiprime, we see that $\mathbb{A}=\mathbb{X} \in \mathcal{S \mathcal { M }}_{N}$ and hence $\mathcal{W}^{\prime}(\boldsymbol{A})$ is type $\mathrm{II}_{1}$ and $\operatorname{dim}(\mathbb{A})=\aleph_{0}$. Further, if $\mathbb{B}=t \odot \mathbb{A}$, then $\mathbb{B}$ is semiminimal (hence $\mathcal{W}^{\prime}(\boldsymbol{B})$ is type $\mathrm{II}_{1}$ ) and the $\mathcal{W}^{*}$-algebras $\mathcal{Z}\left(\mathcal{W}^{\prime}(\boldsymbol{B})\right), \mathcal{Z}\left(\mathcal{W}^{\prime}\left(\aleph_{0} \odot \boldsymbol{B}\right)\right), \mathcal{Z}\left(\mathcal{W}^{\prime}\left(\aleph_{0} \odot \boldsymbol{A}\right)\right)$ and $\mathcal{Z}\left(\mathcal{W}^{\prime}(\boldsymbol{A})\right)$ are isomorphic (since $\aleph_{0} \odot \mathbb{B}=\aleph_{0} \odot \mathbb{A}$ ) which implies that $\mathcal{W}^{\prime}(B)$ is a factor. Consequently, $\mathbb{B}$ is a semiprime. Finally, if $\mathbb{B}$ is a semiprime such that $\mathbb{B} \ll \mathbb{A}$, then from semiminimality of $\mathbb{B}$ it follows that $\frac{1}{n} \odot \mathbb{B} \leqslant \mathbb{A}$ for some natural number $n$. Now the first paragraph of the proof shows that then $\frac{1}{n} \odot \mathbb{B}=\lambda \odot \mathbb{A}$ for some $\lambda>0$ and we are done.

The reader will now easily check that if $\mathbb{A}$ is a prime or a semiprime and $\mathbb{X} \in \mathcal{C D D}_{N}$ is arbitrary, then either $\mathbb{A} \leqslant n \odot \mathbb{X}$ for some natural number $n$ or $\mathbb{A} \perp_{u} \mathbb{X}$. It turns out that much stronger property may be established, similar to a suitable property of prime numbers. Namely:

17.7. Proposition. Let $\left\{\mathbb{X}^{(s)}\right\}_{s \in S} \in \mathcal{C D} \mathcal{D}_{N}$ be a nonempty set and let $\mathbb{A} \leqslant \bigoplus_{s \in S} \mathbb{X}^{(s)}$.

(I) If $\mathbb{A}$ is a prime, there is $s \in S$ such that $\mathbb{A} \leqslant \mathbb{X}^{(s)}$.

(II) Suppose $\mathbb{A}$ is a semiprime. For each $s \in S$ let $\lambda_{s}=\sup \{t \in$ $\left.\mathbb{R}_{+}: t \odot \mathbb{A} \leqslant \mathbb{X}^{(s)}\right\} \in \mathbb{R}_{+} \cup\left\{\aleph_{0}\right\}$. Then $\lambda_{s} \odot \mathbb{A} \leqslant \mathbb{X}^{(s)}(s \in S)$ and $\sum_{s \in S} \lambda_{s} \geqslant 1$. 
Proof. To prove (I), observe that there is $s \in S$ such that $\mathbb{A}$ and $\mathbb{X}^{(s)}$ are not unitarily disjoint. Since $\mathbb{A}$ is a prime, the latter yields that $\mathbb{A} \leqslant \mathbb{X}^{(s)}$.

We now pass to (II). By (VS3) (page 39), $\mathbb{A}^{(s)}:=\lambda_{s} \odot \mathbb{A} \leqslant \mathbb{X}^{(s)}$. Assume that $\lambda_{s}<1$ for every $s \in S$ and $\lambda=\sum_{s \in S} \lambda_{s}<\infty$. By the maximality of $\lambda_{s},\left(\left(1-\lambda_{s}\right) \odot \mathbb{A}=\right) \mathbb{A} \ominus \mathbb{A}^{(s)} \perp_{u} \mathbb{X}^{(s)} \ominus \mathbb{A}^{(s)}=$ : $\mathbb{Y}^{(s)}$ and consequently $\mathbb{A} \perp_{u} \mathbb{Y}^{(s)}$. Thus, $\mathbb{A} \perp_{u} \bigoplus_{s \in S} \mathbb{Y}^{(s)}$. Now since $\bigoplus_{s \in S} \mathbb{X}^{(s)}=\left(\bigoplus_{s \in S} \mathbb{A}^{(s)}\right) \oplus\left(\bigoplus_{s \in S} \mathbb{Y}^{(s)}\right)$, we infer from (PR1) (page 9) that $\mathbb{A} \leqslant \bigoplus_{s \in S} \mathbb{A}^{(s)}$. Further, we see that $\bigvee\left\{\bigoplus_{s \in S^{\prime}} \mathbb{A}^{(s)}: S^{\prime} \in\right.$ $\left.\mathcal{P}_{f}(S)\right\}=\lambda \odot \mathbb{A}$. This combined with Proposition 12.6 (page 33) yields that $\lambda \odot \mathbb{A}=\bigoplus_{s \in S} \mathbb{A}^{(s)}$. So, $\mathbb{A} \leqslant \lambda \odot \mathbb{A}$ and hence $\lambda \geqslant 1$.

Denote by $\mathfrak{a}_{N}, \mathfrak{f}_{N}$ and $\mathfrak{s}_{N}$ the sets of all, respectively, atoms, fractals and semiprimes in $\mathcal{C D D}_{N}$. Further, for $n=1,2, \ldots, \infty$ let $\mathfrak{a}_{N}(n)$ be the set of all atoms of type $I^{n}$. Similarly, we denote by $\mathfrak{s}_{N}(1)$ and $\mathfrak{s}_{N}(\infty)$ the sets of all semiprimes of type $\mathrm{II}^{1}$ and $\mathrm{II}^{\infty}$, respectively. The reader should notice that an atom $\mathbb{A}$ belongs to $\mathfrak{a}_{N}(n)$ for some finite $n$ iff $\operatorname{dim}(\mathbb{A})=n\left(\right.$ and $\mathbb{A} \in \mathfrak{a}_{N}(\infty)$ iff $\left.\operatorname{dim}(\mathbb{A})=\aleph_{0}\right)$. Finally, we put $\mathfrak{p}_{N}=\mathfrak{a}_{N} \cup \mathfrak{f}_{N} \cup \mathfrak{s}_{N}$.

17.8. Proposition. The sets $\mathfrak{a}_{N}(n)(n=1,2, \ldots, \infty), \mathfrak{f}_{N}, \mathfrak{s}_{N}(1)$ and $\mathfrak{s}_{N}(\infty)$ have power $2^{\aleph_{0}}$. Each of these sets contains a subset of power $2^{\aleph_{0}}$ consisting of mutually unitarily disjoint $N$-tuples.

Proof. Let us first justify that each of the sets $\mathfrak{a}_{1}(n), \mathfrak{f}_{1}, \mathfrak{s}_{1}(1)$ and $\mathfrak{s}_{1}(\infty)$ contains at least one bounded nonzero operator. For $\mathfrak{a}_{1}(n)$ this is clear, while for $\mathfrak{f}_{1}, \mathfrak{s}_{1}(1)$ and $\mathfrak{s}_{1}(\infty)$ this follows from the existence of factors of each type and the results on generators of such factors [37], [11] (the same was in fact observed by Ernest, cf. [9, Proposition 1.30]).

Now let $T$ be a bounded nonzero operator of a suitable type (here by a type we mean an atom of type $\mathrm{I}^{n}$, a fractal or a semiprime of type $\left.\mathrm{II}^{n}\right)$. Notice that then $\left\{(r T, \ldots, r T) \in \mathrm{CDD}_{N}: r \in(0, \infty)\right\}$ is a family of mutually unitarily disjoint $N$-tuples of the same type as $T$ (indeed, if $\mathbb{X}$ is a bounded semiprime, then $\|t \odot \mathbb{X}\|=\|\mathbb{X}\|$ for each $t \in \mathbb{R}_{+} \backslash\{0\}$ and thus $r \boldsymbol{X} \perp_{u} s \boldsymbol{X}$ for distinct $r$ and $\left.s\right)$. This proves the second claim of the proposition. To show the first one, it suffices to apply Lemma 9.1 (page 22) and observe that if $\mathbb{X}$ is a semiprime, then $\operatorname{card}\left(\left\{\mathbb{Y} \in \mathfrak{s}_{N}: \mathbb{Y} \not \not_{u} \mathbb{X}\right\}\right)=\operatorname{card}\left(\left\{t \odot \mathbb{X}: t \in \mathbb{R}_{+} \backslash\{0\}\right\}\right)=2^{\aleph_{0}}$

As an immediate consequence of Proposition 17.8 we obtain the following result, announced in Remark 9.9 (page 24).

17.9. Corollary. For $i=\{I, I I, I I I\}, \operatorname{dim}\left(\mathbb{J}_{i}\right)=2^{\aleph_{0}}$.

Denote by $\mathcal{J}^{d}$ the ideal generated by $\mathfrak{p}_{N}$ and let $\mathcal{J}^{c}=\left(\mathcal{J}^{d}\right)^{\perp}$. In other words, $\mathbb{A} \in \mathcal{J}^{d}$ if $\mathbb{A}=\bigoplus_{\mathbb{X} \in \mathfrak{p}_{N}} \beta_{\mathbb{X}} \odot \mathbb{X}$ for some family $\left\{\beta_{\mathbb{X}}\right\}_{\mathbb{X} \in \mathfrak{p}_{N}} \subset$ Card; and $\mathbb{A} \in \mathcal{J}^{c}$ if $\mathbb{B} \notin \mathfrak{p}_{N}$ for every $\mathbb{B} \leqslant \mathbb{A}$. Similarly, whenever $\mathcal{A}$ is an ideal in $\mathcal{C D D}_{N}, \mathcal{A}^{d}$ and $\mathcal{A}^{c}$ denote, respectively, the ideals $\mathcal{A} \cap \mathfrak{J}^{d}$ and 
$\mathcal{A} \cap \mathcal{J}^{c} . \mathcal{A}^{d}$ and $\mathcal{A}^{c}$ are called the discrete and continuous parts of $\mathcal{A}$. For example, we shall write $\mathcal{J}_{I I I}^{c}, \mathcal{J}_{I_{1}}^{d}$, etc. We also define the discrete and continuous parts of every member of $\mathcal{C D D}_{N}$ and each clopen set in $\Omega: \mathbb{X}^{d}=\mathbb{E}\left(\mathbb{X} \mid \mathcal{J}^{d}\right)$ and $\mathbb{X}^{c}=\mathbb{E}\left(\mathbb{X} \mid \mathcal{J}^{c}\right)$ for $\mathbb{X} \in \mathcal{C D D}_{N} ; \Omega^{d}=\operatorname{supp}_{\Omega} \mathcal{J}^{d}$ and $\Omega^{c}=\operatorname{supp}_{\Omega} \mathcal{J}^{c}$; and $E^{d}=E \cap \Omega^{d}$ and $E^{c}=E \cap \Omega^{c}$ for a clopen set $E \subset \Omega$. We should underline that classically the terms discrete and continuous as kinds of operators mean type $I$ and without type I parts, respectively (as it is practised e.g. by Ernest, see [9, Definition 1.22]).

It may be easily checked that $\mathbb{A} \in \mathfrak{p}_{N}$ iff $\widehat{\mathbb{A}}$ has the form $\widehat{\mathbb{A}}=c \cdot j_{\{x\}}$ where either $c=1$ and $x \in \Omega_{I}$ or $c \in \mathbb{R}_{+} \backslash\{0\}$ and $x \in \Omega_{I I}$, or $c=\aleph_{0}$ and $x \in \Omega_{I I I}$. Therefore $\Omega^{d}$ is the closure of the set of all isolated points of $\Omega$. Consequently, we infer from Lemma 14.1 (page 40) and Proposition 17.8 that

17.10. Proposition. Each of the spaces $\Omega_{I_{n}}^{d}(n=1,2, \ldots, \infty), \Omega_{I I_{1}}^{d}$, $\Omega_{I I \infty}^{d}$ and $\Omega_{I I I}^{d}$ is the Cech-Stone compactification of the discrete space of cardinality $2^{\aleph_{0}}$.

Proposition 17.10 and the next two results will be used later to classify ideals in $\mathcal{C D D}_{N}$ up to isomorphism (see Section 23 for definition and details).

17.11. Proposition. Every nonempty clopen set $E \subset \Omega^{c}$ with $c(E)=$ $\aleph_{0}$ is homeomorphic to the Gelfand spectrum of $L^{\infty}([0,1])$.

Proof. There is (unique) nontrivial $\mathbb{A} \in \mathcal{C D} \mathcal{D}_{N}$ such that $\mathbb{A} \leqslant^{s} \mathbb{J}$ and $\operatorname{supp} \widehat{\mathbb{A}}=E$. Since $E \subset \Omega^{c}$ and $c(E)=\aleph_{0}$,

$$
\mathbb{A} \in \mathcal{J}^{c} \quad \text { and } \quad \operatorname{dim}(\mathbb{A})=\aleph_{0} .
$$

Further, since $\mathcal{Z}\left(\mathcal{W}^{\prime}(\boldsymbol{J})\right)$ is isomorphic to $\mathcal{C}(\Omega), \mathcal{Z}\left(\mathcal{W}^{\prime}(\boldsymbol{A})\right)$ is isomorphic to $\mathcal{C}(E)$ (because $\left.\mathbb{A} \leqslant^{s} \mathbb{J}\right)$. The latter means that $E$ is the Gelfand spectrum of $\mathcal{Z}\left(\mathcal{W}^{\prime}(A)\right)$. Now the assertion easily follows from (17-1) and Theorem III.1.22 of [34]. (The last mentioned result asserts that every commutative von Neumann algebra acting on a separable Hilbert space which has no nonzero minimal projections is isomorphic to $L^{\infty}([0,1])$.)

Now for a clopen set $E \subset \Omega$ let $\kappa_{d}(E)$ be the power of the set of all isolated points of $E$ and let $\kappa_{c}(E)=c_{*}\left(E^{c}\right)$. Additionally, let us denote by $D(\mathfrak{m})$ the discrete space of cardinality $\mathfrak{m}$ and by $\mathfrak{X}$ the Gelfand spectrum of $L^{\infty}([0,1])$. Recall that for every completely regular topological space $X, \beta X$ stands for the Cech-Stone compactification of $X$.

17.12. Theorem. Any clopen set $E \subset \Omega$ is homeomorphic to the topological disjoint union of $\beta D\left(\kappa_{d}(E)\right)$ and $\beta\left[D\left(\kappa_{c}(E)\right) \times \mathfrak{X}\right]$.

Proof. Thanks to Lemma 15.7 (page 50) and Proposition 17.11, $E^{c}$ contains an open dense subset homeomorphic to $D\left(\kappa_{c}(E)\right) \times \mathfrak{X}$. Now 
it suffices to apply Lemma 14.1 (page 40) to infer that $E^{d}$ and $E^{c}$ are homeomorphic to, respectively, $\beta D\left(\kappa_{d}(E)\right)$ and $\beta\left[D\left(\kappa_{c}(E)\right) \times \mathfrak{X}\right]$.

17.13. Example. It is clear that $\mathfrak{a}_{N}(1)$ is the collection of all $N$-tuples acting on a one-dimensional Hilbert space. So, $\mathfrak{a}_{N}(1)$ may naturally be identified with $\mathbb{C}^{N}$.

One may also easily check that $\mathfrak{a}_{N}(2)$ consists of all $N$-tuples acting on a two-dimensional Hilbert space which are not of type $\mathcal{J}_{1}$. In other words, if $\boldsymbol{A}=\left(A_{1}, \ldots, A_{N}\right)$ where $A_{1}, \ldots, A_{N}$ are 2 by 2 matrices, then $\mathbb{A} \in \mathfrak{a}_{N}(2)$ iff $A_{j} A_{k}^{*} \neq A_{k}^{*} A_{j}$ for some $j, k \in\{1, \ldots, N\}$.

For $n \geqslant 3$ the characterization of members of $\mathfrak{a}_{N}(n)$ is much more complicated.

\section{Strongly UNITARILY DISJOINT FAMILIES}

Thanks to (BT3) (page 8) and suitable characterizations of the kinds of $N$-tuples appearing below, we see that for every $\mathbb{X} \in \mathcal{C D D}_{N}$ the following equivalences hold true:

$\mathbb{X}$ is type $I, I^{n}, I I, I I^{1}, I I^{\infty}, I I I$, minimal, multiplicity free, a hereditary idempotent, semiminimal, a prime, an atom, a fractal or a semiprime iff so is $\mathfrak{b}(\mathbb{X})$.

However, so far there was no need to use the $\mathfrak{b}$-transform, beside Theorem 3.4 (page 8). From now on, this transform will intensively be involved and without it the presentation would be much more complicated.

We say that two classes $\mathcal{A}, \mathcal{B} \subset \mathrm{CDD}_{N}$ are unitarily disjoint iff $\mathcal{A} \perp_{u}$ $\mathcal{B}$, that is, if $A \perp_{u} B$ for any $A \in \mathcal{A}$ and $B \in \mathcal{B}$. We begin with a classical

18.1. Proposition. Let $\boldsymbol{A}, \boldsymbol{B} \in \mathrm{CDD}_{N}$ be nontrivial $N$-tuples and let $\boldsymbol{X}=\boldsymbol{A} \oplus \boldsymbol{B}$. The following conditions are equivalent:

(i) $A \perp_{u} B$,

(ii) $\mathcal{W}^{\prime}(\boldsymbol{X})=\left\{S \oplus T: S \in \mathcal{W}^{\prime}(\boldsymbol{A}), T \in \mathcal{W}^{\prime}(\boldsymbol{B})\right\}=: \mathcal{W}^{\prime}(\boldsymbol{A}) \oplus \mathcal{W}^{\prime}(\boldsymbol{B})$,

(iii) $I \oplus 0 \in \mathcal{W}^{\prime \prime}(X)$ (where $I$ is the identity operator on $\overline{\mathcal{D}}(\boldsymbol{A})$ and 0 is the zero operator on $\overline{\mathcal{D}}(\boldsymbol{B}))$.

Proof. Using $\mathfrak{b}$-transform and taking into account properties (BT3)(BT5) (page 8), we may assume that $\boldsymbol{A}$ and $\boldsymbol{B}$ are bounded. In that case the equivalence of (i) and (ii) follows from Schur's lemma (cf. Theorem 1.5 in [9]; see also Corollary 1.8 there). Further, (ii) easily implies (iii), since $I \oplus 0$ commutes with every member of $\mathcal{W}^{\prime}(A) \oplus$ $\mathcal{W}^{\prime}(B)$. Finally, if (iii) is satisfied, then all elements of $\mathcal{W}^{\prime}(X)$ commute with $I \oplus 0$ and thus are of the form $S \oplus T$. It is now easily verified that $S \oplus T$ commutes with each entry of $X$ if and only if $S \in \mathcal{W}^{\prime}(A)$ and $T \in \mathcal{W}^{\prime}(B)$.

We are mainly interested in the equivalence of (i) and (iii) in Proposition 18.1. 
Adapting the concept due to Ernest [9] (see Definition 1.31 and §5.7.f there, especially notes on page 187 there), let us consider the free complex algebra $F=F\left(z_{1}, \ldots, z_{N} ; w_{1}, \ldots, w_{N}\right)$ on $2 N$ non-commuting variables $z_{1}, \ldots, z_{N}, w_{1}, \ldots, w_{N}$. Each member of $F$ may naturally be identified with a polynomial in $2 N$ non-commuting variables. Let $*$ be a unique involution on the algebra $F$ such that $z_{j}^{*}=w_{j}$ for $j=1, \ldots, N$. We denote by $\mathcal{P}(N)$ obtained in this way *-algebra equipped with a norm gived by

$$
\left\|p\left(z_{1}, \ldots, z_{N} ; z_{1}^{*}, \ldots, z_{N}^{*}\right)\right\|=\sup _{\left\|T_{j}\right\| \leqslant 1}\left\|p\left(T_{1}, \ldots, T_{N} ; T_{1}^{*}, \ldots, T_{N}^{*}\right)\right\|
$$

where the supremum is taken over all $N$-tuples of contractions acting on a (common, arbitrary) Hilbert space. It follows from the definition that for every $p \in \mathcal{P}(N)$ and $\boldsymbol{X} \in \mathrm{CDD}_{N}$ with $\|\boldsymbol{X}\| \leqslant 1,\left\|p\left(\boldsymbol{X}, \boldsymbol{X}^{*}\right)\right\| \leqslant\|p\|$. The following is left as an easy exercise (use the separability of $\mathcal{P}(N)$ ).

18.2. Lemma. There is a sequence $\left\{\boldsymbol{M}_{n}\right\}_{n=1}^{\infty}$ of atoms in $\mathrm{CDD}_{N}$ acting on finite-dimensional Hilbert spaces such that $\left\|\boldsymbol{M}_{n}\right\| \leqslant 1(n \geqslant 1)$ and for every $p \in \mathcal{P}(N)$,

$$
\|p\|=\sup _{n \geqslant 1}\left\|p\left(\boldsymbol{M}_{n}, \boldsymbol{M}_{n}^{*}\right)\right\| .
$$

With use of the above result and Kaplansky's density theorem [20] (cf. [18, Theorem 5.3.5], [34, Theorem II.4.8], [29, Theorem 1.9.1]) we shall now prove a result which is a starting point for our further investigations. By $\mathcal{P}_{1}(N)$ we denote the closed unit ball of $\mathcal{P}(N)$. Everywhere below $I$ and 0 denote the identity and zero operators on suitable Hilbert spaces. Recall that a net $\left(T_{\sigma}\right)_{\sigma \in \Sigma}$ of bounded operators acting on a Hilbert space $\mathcal{H}$ converges $*$-strongly to an operator $T \in \mathcal{B}(\mathcal{H})$ iff for any $x \in \mathcal{H}, T_{\sigma} x \rightarrow T x(\sigma \in \Sigma)$ and $T_{\sigma}^{*} x \rightarrow T^{*} x(\sigma \in \Sigma)$. We shall denote this by $T_{\sigma} \stackrel{* s}{\rightarrow} T$.

18.3. Proposition. (I) Let $\mathcal{A}$ and $\mathcal{B}$ be arbitrary subsets of $\mathrm{CDD}_{N}$. The following conditions are equivalent:

(i) $\mathcal{A}$ and $\mathcal{B}$ are unitarily disjoint,

(ii) there is a net $\left(p_{\sigma}\right)_{\sigma \in \Sigma} \subset \mathcal{P}_{1}(N)$ such that for any $A \in \mathcal{A}$ and $\boldsymbol{B} \in \mathcal{B}, p_{\sigma}\left(\mathfrak{b}(\boldsymbol{A}), \mathfrak{b}(\boldsymbol{A})^{*}\right) \stackrel{* s}{\rightarrow} I$ and $p_{\sigma}\left(\mathfrak{b}(\boldsymbol{B}), \mathfrak{b}(\boldsymbol{B})^{*}\right) \stackrel{* s}{\rightarrow} 0$.

(II) If $\boldsymbol{A}$ and $\boldsymbol{B}$ are two $N$-tuples acting in separable Hilbert spaces, then $\boldsymbol{A} \perp_{u} \boldsymbol{B}$ iff there is a sequence $\left(p_{n}\right)_{n=1}^{\infty} \subset \mathcal{P}_{1}(N)$ such that $p_{n}\left(\mathfrak{b}(\boldsymbol{A}), \mathfrak{b}(\boldsymbol{A})^{*}\right) \stackrel{* s}{\rightarrow} I$ and $p_{n}\left(\mathfrak{b}(\boldsymbol{B}), \mathfrak{b}(\boldsymbol{B})^{*}\right) \stackrel{* s}{\rightarrow} 0$.

Proof. (I): By (BT5) (page 8), (i) is implied by (ii). To prove the converse, assume $\mathcal{A} \perp_{u} \mathcal{B}$. Let $A=\bigoplus\{X: X \in \mathcal{A}\}$ and $B=\bigoplus\{Y: Y \in$ $\mathcal{B}\}$. Thanks to (PR2) (page 9), $\boldsymbol{A} \perp_{u} \boldsymbol{B}$. Further, let $\left\{\boldsymbol{M}_{n}\right\}_{n=1}^{\infty}$ be as in Lemma 18.2. Let $\boldsymbol{M}$ be the direct sum of all $\boldsymbol{M}_{n}$ 's which are unitarily disjoint from $\mathfrak{b}(\boldsymbol{B})\left(\boldsymbol{M}\right.$ is trivial provided $\boldsymbol{M}_{n} \leqslant \mathfrak{b}(\boldsymbol{B})$ for each $\left.n\right)$. Again by (PR2) and (BT5), $\boldsymbol{M} \oplus \mathfrak{b}(\boldsymbol{A}) \perp_{u} \mathfrak{b}(\boldsymbol{B})$. Put $\boldsymbol{X}=(\boldsymbol{M} \oplus \mathfrak{b}(\boldsymbol{A})) \boxplus \mathfrak{b}(\boldsymbol{B})$, 
$\mathcal{H}_{1}=\overline{\mathcal{D}}(\boldsymbol{M} \oplus \mathfrak{b}(\boldsymbol{A}))$ and $\mathcal{H}_{2}=\overline{\mathcal{D}}(\mathfrak{b}(B))$. It follows from our construction that for each $p \in \mathcal{P}(N)$,

$$
\|p\|=\left\|p\left(\boldsymbol{X}, \boldsymbol{X}^{*}\right)\right\| \text {. }
$$

Let $\mathcal{M}=\left\{p\left(\boldsymbol{X}, \boldsymbol{X}^{*}\right): \quad p \in \mathcal{P}(N)\right\} . \mathcal{M}$ is a unital selfadjoint subalgebra of $\mathcal{B}\left(\mathcal{H}_{1} \oplus \mathcal{H}_{2}\right)$. We infer from von Neumann's double commutant theorem [24] ([18, Theorem 5.3.1], [34, Theorem II.3.9], [29, Theorem 1.20.3]) that the closure of $\mathcal{M}$ in the strong operator topology coincides with $\mathcal{W}^{\prime \prime}(\boldsymbol{X})$. Further, (18-1) yields that the closed unit ball in $\mathcal{M}$ coincides with $\left\{p\left(\boldsymbol{X}, \boldsymbol{X}^{*}\right): p \in \mathcal{P}_{1}(N)\right\}$. An application of Proposition 18.1 shows that $I \oplus 0 \in \mathcal{W}^{\prime \prime}(\boldsymbol{X})$ where $I \in \mathcal{B}\left(\mathcal{H}_{1}\right)$ and $0 \in \mathcal{B}\left(\mathcal{H}_{2}\right)$. Finally, Kaplansky's density theorem asserts that there is a net $\left(p_{\sigma}\right)_{\sigma \in \Sigma} \in \mathcal{P}_{1}(N)$ such that $p_{\sigma}\left(\boldsymbol{X}, \boldsymbol{X}^{*}\right) \stackrel{* s}{\rightarrow} I \oplus 0$. Since every member of $\mathcal{A}$ and $\mathcal{B}$ is a summand of $\boldsymbol{A}$ and $\boldsymbol{B}$, respectively, the assertion of (ii) is fulfilled.

To prove (II), repeat the above argument and observe that in that case both $\mathcal{H}_{1}$ and $\mathcal{H}_{2}$ are separable and hence Kaplansky's density theorem asserts the existence of a suitable sequence, since the closed unit ball in $\mathcal{B}(\mathcal{H})$ for separable $\mathcal{H}$ is metrizable in the $*$-strong topology (see e.g. [9, Proposition 2.2]).

Let us now introduce the following

18.4. Definition. Let $\mathcal{A}$ and $\mathcal{B}$ be arbitrary collections (sets or classes) of $N$-tuples. We say that $\mathcal{A}$ and $\mathcal{B}$ are strongly unitarily disjoint, in symbol $\mathcal{A} \perp_{s} \mathcal{B}$, if there is a sequence $\left(p_{n}\right)_{n=1}^{\infty} \subset \mathcal{P}_{1}(N)$ such that $p_{n}\left(\mathfrak{b}(\boldsymbol{A}), \mathfrak{b}(\boldsymbol{A})^{*}\right) \oplus p_{n}\left(\mathfrak{b}(\boldsymbol{B}), \mathfrak{b}(\boldsymbol{B})^{*}\right) \stackrel{* s}{\rightarrow} I \oplus 0$ for any $\boldsymbol{A} \in \mathcal{A}$ and $\boldsymbol{B} \in \mathcal{B}$. Two $N$-tuples $\boldsymbol{X}, \boldsymbol{Y} \in \mathrm{CDD}_{N}$ are strongly unitarily disjoint $\left(\boldsymbol{X} \perp_{s} \boldsymbol{Y}\right)$ provided so are the sets $\{\boldsymbol{X}\}$ and $\{\boldsymbol{Y}\}$.

The reader should easily notice that for two sets $\mathcal{A}$ and $\mathcal{B}$ of $N$ tuples, $\mathcal{A} \perp_{s} \mathcal{B}$ iff $(\bigoplus \mathcal{A}) \perp_{s}(\bigoplus \mathcal{B})$. It is also clear that if $\mathcal{A}$ and $\mathcal{B}$ are strongly unitarily disjoint, then $\mathcal{A} \perp_{u} \mathcal{B}$.

18.5. Remark. Let $\boldsymbol{A}$ and $\boldsymbol{A}^{\prime}$ be two unitarily equivalent $N$-tuples. Observe that then $p\left(\mathfrak{b}(\boldsymbol{A}), \mathfrak{b}(\boldsymbol{A})^{*}\right) \equiv p\left(\mathfrak{b}\left(\boldsymbol{A}^{\prime}\right), \mathfrak{b}\left(\boldsymbol{A}^{\prime}\right)^{*}\right)$ for every $p \in \mathcal{P}(N)$. What is more, for every complex number $\lambda$ and a net $\left(p_{\sigma}\right)_{\sigma \in \Sigma} \subset$ $\mathcal{P}(N), p_{\sigma}\left(\mathfrak{b}(\boldsymbol{A}), \mathfrak{b}(\boldsymbol{A})^{*}\right) \rightarrow \lambda I *$-strongly (strongly, weakly, etc.) iff $p_{\sigma}\left(\mathfrak{b}\left(\boldsymbol{A}^{\prime}\right), \mathfrak{b}\left(\boldsymbol{A}^{\prime}\right)^{*}\right) \rightarrow \lambda I$ in the same topology. This means that for any $\mathbb{A} \in \mathcal{C D} \mathcal{D}_{N}$ and $p \in \mathcal{P}(N), p\left(\mathfrak{b}(\mathbb{A}), \mathfrak{b}(\mathbb{A})^{*}\right)$ is a well defined member of eDD and

$$
p_{\sigma}\left(\mathfrak{b}(\mathbb{A}), \mathfrak{b}(\mathbb{A})^{*}\right) \stackrel{* s}{\rightarrow} \lambda I
$$

is well understood. (We do not write in (18-2) ' $\mathbb{I}$ ' instead of ' $I$ ' because ' $I$ ' represents here the identity operator on a Hilbert space of (suitable) arbitrary dimension. The usage of $\mathbb{I}$ may lead to misunderstandings. In fact, (18-2) expresses only a property of the net $\left.\left\{p_{\sigma}\left(\mathfrak{b}(\mathbb{A}), \mathfrak{b}(\mathbb{A})^{*}\right)\right\}_{\sigma \in \Sigma} \cdot\right)$ 
Consequently, in the samy way as in Definition 18.4 we may define strongly unitarily disjoint subclasses of $\mathcal{C D D}_{N}$. We follow this concept in next sections.

Surely the main problem concerning the strong unitary disjointness is the question of when two unitarily disjoint families of $N$-tuples acting in separable Hilbert spaces are strongly unitarily disjoint. We will not answer this question in this treatise. However, the reader should remember that strong unitary disjointness and unitary disjointness are nonequivalent even for families of $N$-tuples acting on a one-dimensional Hilbert space. Indeed, such $N$-tuples may naturally be identified with points of $\mathbb{C}^{N}$. If $p_{1}, p_{2}, \ldots$ is an arbitrary sequence of members of $\mathcal{P}(N)$ and $\lambda \in \mathbb{C}$, the set $\left\{z \in \mathbb{C}^{N}: p_{n}\left(\mathfrak{b}(z), \mathfrak{b}(z)^{*}\right) \rightarrow \lambda\right\}$ is $\mathcal{F}_{\sigma \delta}$ in $\mathbb{C}^{N}$. Thus, if $A \subset \mathbb{C}^{N}$ is not $\mathcal{F}_{\sigma \delta}, A \perp_{u} \mathbb{C}^{N} \backslash A$ but $A$ and $\mathbb{C}^{N} \backslash A$ are not strongly unitarily disjoint.

The next result is a consequence of Proposition 18.3. We omit its proof.

18.6. Proposition. Let $\mathcal{A}$ and $\mathcal{B}$ be two countable families of $N$ tuples acting in separable Hilbert spaces. Then $\mathcal{A} \perp_{u} \mathcal{B}$ if and only if $\mathcal{A} \perp_{s} \mathcal{B}$.

In the sequel we shall also need the following simple

18.7. Lemma. Let $\boldsymbol{A}$ be a bounded $N$-tuple acting on a separable Hilbert space such that $\|A\| \leqslant 1$. For every $T \in \mathcal{W}(A)$ with $\|T\| \leqslant 1$ there is a sequence $\left(p_{n}\right)_{n=1}^{\infty} \subset \mathcal{P}_{1}(N)$ such that $p_{n}\left(A, A^{*}\right) \stackrel{* s}{\rightarrow} T$.

Proof. We mimic the proof of Proposition 18.3. As there, we see that there is a sequence $\left\{\boldsymbol{M}_{n}\right\}_{n=1}^{\infty}$ of $N$-tuples of contraction matrices such that $\boldsymbol{M}_{n} \perp_{u} \boldsymbol{A}$ for each $n$ and $\|p\|=\| p\left(\boldsymbol{M} \oplus \boldsymbol{A}, \boldsymbol{M}^{*} \oplus\right.$ $\left.\boldsymbol{A}^{*}\right) \|$ for every $p \in \mathcal{P}(N)$ with $\boldsymbol{M}=\bigoplus_{n=1}^{\infty} \boldsymbol{M}_{n}$. Since $\boldsymbol{M} \perp_{u} \boldsymbol{A}$, $\mathcal{W}^{\prime}(\boldsymbol{M} \oplus \boldsymbol{A})=\mathcal{W}^{\prime}(\boldsymbol{M}) \oplus \mathcal{W}^{\prime}(\boldsymbol{A})$ (by Proposition 18.1). Consequently, $\mathcal{W}^{\prime \prime}(\boldsymbol{M}) \oplus \mathcal{W}^{\prime \prime}(\boldsymbol{A}) \subset \mathcal{W}^{\prime \prime}(\boldsymbol{M} \oplus \boldsymbol{A})$ and thus $0 \oplus T \in \mathcal{W}(\boldsymbol{M} \oplus \boldsymbol{A})$. Finally, since $\boldsymbol{M} \oplus \boldsymbol{A}$ acts on a separable Hilbert space, Kaplansky's density theorem finishes the proof (cf. the proof of Proposition 18.3).

18.8. Remark. Let $\mathfrak{P}=\left\{p_{\sigma}\right\}_{\sigma \in \Sigma} \subset \mathcal{P}_{1}(N)$ be any net and let $\lambda \in \mathbb{C}$. Denote by $\mathcal{J}_{\mathfrak{P}}(\lambda)$ the class of all $\mathbb{X} \in \mathcal{C D} \mathcal{D}_{N}$ for which

$$
p_{\sigma}\left(\mathfrak{b}(\mathbb{X}), \mathfrak{b}(\mathbb{X})^{*}\right) \stackrel{* s}{\rightarrow} \lambda I .
$$

One easily checks that $\mathcal{J}_{\mathfrak{P}}(\lambda)$ is an ideal and $\mathcal{J}_{\mathfrak{P}}(\lambda) \perp_{u} \mathcal{J}_{\mathfrak{P}}\left(\lambda^{\prime}\right)$ whenever $\lambda^{\prime} \neq \lambda$. For every subclass $\mathcal{A}$ of $\mathcal{C D D}_{N}$ let $J(\mathcal{A})$ denote the smallest ideal in $\mathcal{C D D}_{N}$ which contains $\mathcal{A}$. The above notice shows that for arbitrary two subclasses $\mathcal{A}$ and $\mathcal{B}$ of $\mathcal{C D} \mathcal{D}_{N}, \mathcal{A} \perp_{s} \mathcal{B}$ iff $J(\mathcal{A}) \perp_{s} J(\mathcal{B})$, iff $\mathbb{J}(J(\mathcal{A})) \perp_{s} \mathbb{J}(J(\mathcal{B}))$. In particular, strong unitary disjointness of sets or classes may always be reduced to the issue of strong unitary disjointness of suitable $N$-tuples $\mathbb{X}$ and $\mathbb{Y}$ such that $\mathbb{X} \leqslant s$ and $\mathbb{Y} \leqslant s \mathbb{~}$. 


\section{MeAsure-THEORETIC PRELIMINARIES}

Our next objective is to propose the prime decomposition of $N$-tuples (Theorem 22.14, page 104). Essentially this will be based on the same idea (that is, on central decompositions of von Neumann algebras) as Ernest's central decomposition of a bounded operator ([9, Chapter 3]). The difference between his and our approaches (beside bigger generality) is the following. Ernest has focused on a single operator $T$ and studied its (nonscalar) spectrum $\widehat{T}$ and quasi-spectrum $\widetilde{T}$. Central decomposition of the operator $T$ 'takes place' in $\widetilde{T}$. Further the author compares operators (and their central decompositions) which have the same quasi-spectra. It seems to us that Ernest's work was inspired by the spectral theorem for a normal operator. Our treatise is inspired by the prime decomposition of natural numbers. Our interpretation is therefore in a more algebraic fashion. Also comparing Ernest's work and our, we may say that his approach is local, while our is global.

The road to Prime Decomposition Theorem is long because of measure-theoretic technicalities. First we shall define the Borel structure on $\mathcal{C D} \mathcal{D}_{N}$ (this is done in this part), next we shall generalize the notion of a direct integral to the context of $N$-tuples (Section 20) to define 'continuous' direct sums (Section 21) among which we shall distinguish regular ones (which deal with unitary disjointness) and finally we shall show that every member of $\mathcal{C D D}_{N}$ admits an (in a sense) unique regular prime decomposition (Section 22).

The concept of direct integrals (of Hilbert spaces, operators, von Neumann algebras, etc.) is essentially due to von Neumann and is widely discussed in many classical textbooks on von Neumann algebras. Here we shall focus on main ideas and many proofs will be omitted. The reader interested in details should consult e.g. Chapters 2 and 3 of [9]; [6, 7]; [19, Chapter 14]; §IV.8, §V.6 and Appendix in [34]; [29, Chapter 3]; [30, Chapter I]; or the original paper by von Neumann [25]. It is also assumed that the reader is well oriented in basics of measure theory as well as in aspects of reduction theory of von Neumann algebras.

Measurable sets (i.e. elements of a given $\sigma$-algebra) will also be called Borel. Everywhere below by a measurable or Borel function of a measurable space $(X, \mathfrak{M})$ into a measurable space $(Y, \mathfrak{N})$ we mean any function $f: X \rightarrow Y$ such that $f^{-1}(B) \in \mathfrak{M}$ for any $B \in \mathfrak{N}$. The function $f$ is a Borel isomorphism if $f$ is a bijection and $f$ and $f^{-1}$ are measurable. For two measures $\mu$ and $\nu$ defined on a common $\sigma$-algebra $\mathfrak{M}$ we shall write $\mu \ll \nu$ iff $\mu$ is absolutely continuous with respect to $\nu$ and we call $\mu$ and $\nu$ (mutually) singular iff $\mu \perp \nu$, i.e. if $\mu$ and $\nu$ are concetrated on disjoint measurable sets. If $A \in \mathfrak{M},\left.\mu\right|_{A}$ denotes the measure on $\mathfrak{M}$ given by $\left.\mu\right|_{A}(B)=\mu(A \cap B)$. For a topological space $X, \mathfrak{B}(X)$ stands for the smallest $\sigma$-algebra containing all open 
subsets of $X$. Following Takesaki [34, Appendix], we call a measurable space $(X, \mathfrak{M})$ a standard Borel space iff $(X, \mathfrak{M})$ is Borel isomorphic to $(Y, \mathfrak{B}(Y))$ where $Y$ is a Borel subset of a separable complete metric space. Equivalently, $(X, \mathfrak{M})$ is standard iff $(X, \mathfrak{M})$ is Borel isomorphic to $(A, \mathfrak{B}(A))$ where $A$ is a countable (finite or not) subset of $[0,1]$ or $A=[0,1]$ (cf. [34, Corollary A.11]). If $(X, \mathfrak{M})$ and $(Y, \mathfrak{N})$ are standard Borel spaces and $f: X \rightarrow Y$ is measurable, then $(X \times Y, \mathfrak{M} \otimes \mathfrak{N})$ is a standard Borel space as well and $\Gamma(f) \in \mathfrak{M} \otimes \mathfrak{N}$ where $\Gamma(f)=$ $\{(x, f(x)): x \in X\}$ is the graph of $f$. The space $(X, \mathfrak{M})$ is SouslinBorel iff it is the image of a standard Borel space under a Borel function and $X$ is countably separated (the latter means that there are sets $E_{1}, E_{2}, \ldots \in \mathfrak{M}$ such that for any two distinct points $x$ and $y$ of $X$ there is $n$ with $\left.\operatorname{card}\left(\{x, y\} \cap E_{n}\right)=1\right)$. In what follows, we shall often identify $I_{\aleph_{0}}$ with $[0, \infty]$.

Let $(X, \mathfrak{M}, \mu)$ be a measure space ( $\mu$ need not be $\sigma$-finite nor complete). We denote by $\mathcal{N}(\mu)$ the null $\sigma$-ideal in $\mathfrak{M}$ induced by $\mu$, that is, $\mathcal{N}(\mu)=\{A \in \mathfrak{M}: \quad \mu(A)=0\} . \quad(X, \mathfrak{M}, \mu)$ is said to be a standard measure space (or, equivalently, $\mu$ is standard) iff $\mu$ is nonzero $\sigma$-finite and $X \backslash Z$ is a standard Borel space for some $Z \in \mathcal{N}(\mu)$. By [34, Corollary A.14], every $\sigma$-finite measure on a Souslin-Borel space is standard.

For $n=1,2, \ldots$ let $\mathcal{H}_{n}$ be a fixed Hilbert space of dimension $n$ and let $\mathcal{H}_{\infty}$ be a fixed separable infinite-dimensional Hilbert space (these spaces are fixed for this and the next two sections). Further, let $\mathcal{H}$ denote one of the spaces $\mathcal{H}_{1}, \mathcal{H}_{2}, \ldots, \mathcal{H}_{\infty}$. The norm and the weak topologies of $\mathcal{H}$ induces the same $\sigma$-algebra on $\mathcal{H}$ which is for us the default Borel structure of $\mathcal{H}$. Similarly, the $*$-strong, strong and weak operator topologies induces the same Borel structures on $\mathcal{B}(\mathcal{H})$. In other words, the $\sigma$-algebra $\mathfrak{W}_{\mathcal{H}}$ generated by all open sets with respect to any of these topologies is independent of the topology we choose. Moreover, $\left(\mathcal{B}(\mathcal{H}), \mathfrak{W}_{\mathcal{H}}\right)$ is a standard Borel space, which means that $\left(\mathcal{B}(\mathcal{H}), \mathfrak{W}_{\mathcal{H}}\right)$ is isomorphic as a measurable space to $([0,1], \mathfrak{B}([0,1]))$. The addition and multiplication are measurable as functions of $\left(\mathcal{B}(\mathcal{H}) \times \mathcal{B}(\mathcal{H}), \mathfrak{W}_{\mathcal{H}} \otimes \mathfrak{W}_{\mathcal{H}}\right)$ into $\left(\mathcal{B}(\mathcal{H}), \mathfrak{W}_{\mathcal{H}}\right)$ and the functions $T \mapsto T^{*}, T \mapsto|T|, T \mapsto Q_{T}$ and $T \mapsto T^{-1}$ are measurable as well (the last mentioned function comes from the set of all invertible operators which is measurable).

The following result will enable us to define a Borel structure on the set $\operatorname{CDD}(\mathcal{H})$.

19.1. Lemma. The open unit ball $B$ of $\mathcal{B}(\mathcal{H})$ and the set $\mathfrak{b}(\mathcal{H})$ of all $T \in \mathcal{B}(\mathcal{H})$ such that $\|T x\|<\|x\|$ for any nonzero $x \in \mathcal{H}$ are measurable. The $\mathfrak{b}$-transform is an isomorphism between measurable spaces $\mathcal{B}(\mathcal{H})$ and $B$.

Proof. We shall only explain why $\mathfrak{b}(\mathcal{H})$ is measurable. Notice that $T \in \mathfrak{b}(\mathcal{H})$ iff $\mathcal{N}\left(I-T^{*} T\right)$ is trivial. Now if $P_{T}$ denotes the orthogonal 
projection onto $\mathcal{N}\left(I-T^{*} T\right)$, then the function $T \mapsto P_{T}$ is measurable, thanks to [9, Proposition 2.4], and we are done.

Since the $\mathfrak{b}$-transform establishes a one-to-one correspondence between members of $\operatorname{CDD}(\mathcal{H})$ and $\mathfrak{b}(\mathcal{H})$, we may introduce

19.2. Definition. The Borel structure of $\operatorname{CDD}(\mathcal{H})$ is the unique Borel structure which makes the $\mathfrak{b}$-transform an isomorphism. In other words, a set $F \subset \operatorname{CDD}(\mathcal{H})$ is measurable, in symbol $F \in \mathfrak{B}(\operatorname{CDD}(\mathcal{H}))$, iff $\{\mathfrak{b}(X): X \in F\} \in \mathfrak{W}_{\mathcal{H}}$.

Lemma 19.1 implies that $\operatorname{CDD}(\mathcal{H})$ is a standard Borel space, that $\mathcal{B}(\mathcal{H})$ is a measurable subset of $\operatorname{CDD}(\mathcal{H})$ and that the original Borel structure of $\mathcal{B}(\mathcal{H})$ coincides with the one inherited from the Borel structure of $\operatorname{CDD}(\mathcal{H})$.

Recall that $\operatorname{CDD}_{N}(\mathcal{H})=\operatorname{CDD}(\mathcal{H})^{N}$. We equip $\operatorname{CDD}_{N}(\mathcal{H})$ with the product $\sigma$-algebra $\mathfrak{B}\left(\operatorname{CDD}_{N}(\mathcal{H})\right)=\mathfrak{B}(\operatorname{CDD}(\mathcal{H})) \otimes \ldots \otimes \mathfrak{B}(\operatorname{CDD}(\mathcal{H}))$. Observe that $\operatorname{CDD}_{N}(\mathcal{H})$ is a standard Borel space and the $\mathfrak{b}$-transform is an isomorphism of the measurable space $\operatorname{CDD}_{N}(\mathcal{H})$ onto a measurable set $\mathfrak{b}(\mathcal{H})^{N}$. Moreover, it follows from suitable properties of the $\mathfrak{b}$-transform that each of the functions $X \mapsto X^{*}, X \mapsto|X|$ and $\boldsymbol{X} \mapsto \boldsymbol{Q}_{\boldsymbol{X}}$ (of $\mathrm{CDD}_{N}(\mathcal{H})$ into itself) are measurable.

Now let $\mathcal{S} \mathcal{E} \mathcal{P}_{N}$ be the set of all $\mathbb{A} \in \mathcal{C D} \mathcal{D}_{N}$ such that $0<\operatorname{dim}(\mathbb{A}) \leqslant$ $\aleph_{0}$. Observe that the function $\Phi: \bigcup_{n=1}^{n=\infty} \operatorname{CDD}_{N}\left(\mathcal{H}_{n}\right) \ni X \mapsto \mathbb{X} \in$ $\mathcal{S} \mathcal{E} \mathcal{P}_{N}$ is a surjection. We define a $\sigma$-algebra $\mathfrak{B}_{N}$ on $\mathcal{S} \mathcal{E} \mathcal{P}_{N}$ by the rule: $\mathcal{F} \in \mathfrak{B}_{N}$ iff for every $n \in\{1,2,3, \ldots, \infty\}, \Phi^{-1}(\mathcal{F}) \cap \operatorname{CDD}_{N}\left(\mathcal{H}_{n}\right) \in$ $\mathfrak{B}\left(\operatorname{CDD}_{N}\left(\mathcal{H}_{n}\right)\right)$. It is obviously seen that the definition of $\mathfrak{B}_{N}$ is independent of the choice of $\mathcal{H}_{n}$ 's. For every $\mathcal{A} \in \mathfrak{B}_{N}$ we shall denote by $\mathfrak{B}(\mathcal{A})$ the $\sigma$-algebra of all sets $\mathcal{B} \in \mathfrak{B}_{N}$ contained in $\mathcal{A}$.

As it was shown by Ernest (see [9, Corollary 2.33]), $\mathcal{S} \mathcal{E} \mathcal{P}_{N}$ is not countably separated. This causes that investigating of the Borel structure of $\mathcal{S E \mathcal { P }}{ }_{N}$ is difficult and complicated. The rest of this section is devoted to establish measurability of some (important for us) sets and functions. For $n=1,2, \ldots, \infty$ let $\mathcal{S E P}_{N}(n)$ consist of all $\mathbb{A} \in$ $\mathcal{S E P}_{N}$ with $\operatorname{dim}(\mathbb{A})=n$. It follows from the definition of $\mathfrak{B}_{N}$ that $\mathcal{S E P}_{N}(n) \in \mathfrak{B}_{N}$ for every $n$. When $n$ is finite, much more can be said about $\mathcal{S E P}_{N}(n)$ (cf. Proposition 2.46 and Corollary 2.47 in [9]). Namely,

19.3. Proposition. For every finite $n, \mathcal{S E P}_{N}(n)$ is a standard Borel space and there are a Borel set $S_{n} \subset \mathrm{CDD}_{N}\left(\mathcal{H}_{n}\right)$ and a Borel isomorphism $\chi_{n}: \mathcal{S E \mathcal { P }}_{N}(n) \ni \mathbb{A} \mapsto T_{\mathbb{A}} \in S_{n}$ such that $\boldsymbol{T}_{\mathbb{A}}$ is a representative of $\mathbb{A}$ for every $\mathbb{A}$.

Proof. It is clear that $\mathcal{C D} \mathcal{D}_{N}\left(\mathcal{H}_{n}\right)$ coincides with the space $M_{n}^{N}$ of all $N$-tuples of $n \times n$ matrices. Let $\pi: M_{n}^{N} \rightarrow \mathcal{S} \mathcal{E} \mathcal{P}_{N}(n)$ be the quotient map (i.e. $\pi(\boldsymbol{X})=\mathbb{X}$ ). Equip $\mathcal{S} \mathcal{E} \mathcal{P}_{N}(n)$ with the quotient topology (induced by $\pi$ ). Since the unitary group of $n \times n$ matrices is compact, 
$\mathcal{S E P}_{N}(n)$ is locally compact and $\pi$ is a proper continuous mapping. What is more, $\mathcal{S E P}_{N}(n)$ is separable and metrizable. It is now clear that the $\sigma$-algebra generated by all open sets coincides with the one inherited from $\mathfrak{B}_{N}$. This yields that $\mathcal{S} \mathcal{E} \mathcal{P}_{N}(n)$ is a standard Borel space. The existence of $S_{n}$ and $\chi_{n}$ may easily be deduced e.g. from [22, Corollary XIV.2.1] applied to the partition $\left\{\pi^{-1}(\{\mathbb{X}\}): \mathbb{X} \in \mathcal{S} \mathcal{E} \mathcal{P}_{N}(n)\right\}$, or from [22, Corollary XIV.1.1] (see also [4]) applied to the multifunction $\mathcal{S E P}_{N}(n) \ni \mathbb{X} \mapsto \pi^{-1}(\{\mathbb{X}\}) \subset M_{n}$.

We are now mainly interested in the Borel structure of $\mathcal{S} \mathcal{E} \mathcal{P}_{N}(\infty)$. However, in some arguments we shall need to work also with $N$-tuples acting on finite-dimensional Hilbert spaces and therefore below we explore $\operatorname{CDD}_{N}\left(\mathcal{H}_{\infty}\right)$ as well as $\operatorname{CDD}_{N}\left(\mathcal{H}_{n}\right)$ with finite $n$. Since our main interest are primes and semiprimes, we may restrict our considerations to factor $N$-tuples defined below. Similar results to those presented below the reader may find in Chapter 2 of [9].

As before, $\mathcal{H}$ denotes one of the spaces $\mathcal{H}_{1}, \mathcal{H}_{2}, \ldots, \mathcal{H}_{\infty}$. The functions $\mathrm{CDD}_{N}(\mathcal{H}) \ni \boldsymbol{X} \mapsto \mathcal{W}^{\prime \prime}(\boldsymbol{X}) \in \mathscr{W}(\mathcal{H})$ and $\mathrm{CDD}_{N}(\mathcal{H}) \ni \boldsymbol{X} \mapsto$ $\mathcal{W}^{\prime}(X) \in \mathscr{W}(\mathcal{H})$ are measurable when $\mathscr{W}(\mathcal{H})$ denotes the collection of all von Neumann subalgebras of $\mathcal{B}(\mathcal{H})$ and is equipped with the Effros Borel structure $[6,7]$ (cf. [9, page 54] combined with Theorem IV.8.4 and Corollary IV.8.6 in [34]). Consequently, the following sets are measurable subsets of $\operatorname{CDD}_{N}(\mathcal{H})$ (compare with notes on page 55 of [9]; [34, Theorem V.6.6] and [26]):

- the set of all atoms $\mathfrak{a}_{N}(\mathcal{H})=\left\{A \in \mathrm{CDD}_{N}(\mathcal{H}): \mathbb{A} \in \mathfrak{a}_{N}\right\}$,

- the set of all fractals $\mathfrak{f}_{N}(\mathcal{H})=\left\{A \in \mathrm{CDD}_{N}(\mathcal{H}): \mathbb{A} \in \mathfrak{f}_{N}\right\}$,

- the set of all semiprimes $\mathfrak{s}_{N}(\mathcal{H})=\left\{A \in \mathrm{CDD}_{N}(\mathcal{H}): \mathbb{A} \in \mathfrak{s}_{N}\right\}$,

- the set of all factor $N$-tuples

$$
\mathfrak{F}_{N}(\mathcal{H})=\left\{A \in \mathrm{CDD}_{N}(\mathcal{H}): \mathcal{W}^{\prime \prime}(A) \text { is a factor }\right\},
$$

- the sets of all factor $N$-tuples of type I, $\mathrm{I}^{n}, \mathrm{II}, \mathrm{II}^{1}, \mathrm{II}^{\infty}$ and III.

(The above properties imply that $\mathfrak{a}_{N}, \mathfrak{f}_{N}, \mathfrak{s}_{N}$ as well as $\mathfrak{F}_{N}:=\{\mathbb{F} \in$ $\mathcal{S E P}_{N}: \mathcal{W}^{\prime \prime}(\boldsymbol{F})$ is a factor $\}$ are members of $\mathfrak{B}_{N}$. When $\mathcal{H}$ is finitedimensional, $\mathfrak{s}_{N}(\mathcal{H})$ and $\mathfrak{f}_{N}(\mathcal{H})$ are of course empty.) We infer from Proposition 17.2 (page 59) that for every $\boldsymbol{F} \in \mathfrak{F}_{N}(\mathcal{H}) \backslash\left(\mathfrak{a}_{N}(\mathcal{H}) \cup \mathfrak{f}_{N}(\mathcal{H}) \cup\right.$ $\mathfrak{s}_{N}(\mathcal{H})$ ) either there exist a unique $n \in\left\{2,3,4, \ldots, \aleph_{0}\right\}$ and a unique $\mathbb{A} \in \mathfrak{a}_{N}$ such that $\mathbb{F}=n \odot \mathbb{A}$ or there is (nonunique) $\mathbb{A} \in \mathfrak{s}_{N}$ for which $\mathbb{F}=\aleph_{0} \odot \mathbb{A}$.

Everywhere below $n$ and $m$ represent positive integer as well as $\infty$.

The following result appears in [9] as Corollary 2.11. Below we give a shorter proof.

\subsection{Lemma. The set}

$$
\mathfrak{D}_{N}(n, m)=\left\{(\boldsymbol{A}, \boldsymbol{B}) \in \mathrm{CDD}_{N}\left(\mathcal{H}_{n}\right) \times \mathrm{CDD}_{N}\left(\mathcal{H}_{m}\right): \boldsymbol{A} \perp_{u} \boldsymbol{B}\right\}
$$

is measurable (i.e. $\mathfrak{D}_{N}(n, m) \in \mathfrak{B}\left(\mathrm{CDD}_{N}\left(\mathcal{H}_{n}\right)\right) \otimes \mathfrak{B}\left(\mathrm{CDD}_{N}\left(\mathcal{H}_{m}\right)\right)$ ). 
Proof. Let $\mathcal{K}=\mathcal{H}_{\infty}$ and let $U_{j}: \aleph_{0} \odot \mathcal{H}_{j} \rightarrow \mathcal{K}$ be unitary $\left(\aleph_{0} \odot \mathcal{H}_{j}\right.$ symbolizes the Hilbert space in which act $N$-tuples of the form $\aleph_{0} \odot \boldsymbol{X}$ with $\left.X \in \operatorname{CDD}_{N}\left(\mathcal{H}_{j}\right)\right)$. Let $\mathcal{Q}$ be the set of all $p \in \mathcal{P}$ such that $\|p\| \leqslant 2$ and all coefficients of $p$ belong to $\mathbb{Q}+i \mathbb{Q}$. It may be deduced from Proposition 18.1 (page 64) and Lemma 18.7 (page 67) that $\boldsymbol{A} \perp_{u} \boldsymbol{B}$ where $\boldsymbol{A} \in \mathrm{CDD}_{N}\left(\mathcal{H}_{n}\right)$ and $\boldsymbol{B} \in \mathrm{CDD}_{N}\left(\mathcal{H}_{m}\right)$ iff there is a sequence $\left(p_{k}\right)_{k=1}^{\infty} \subset \mathcal{Q}$ such that $U_{n} p_{k}\left(\mathfrak{b}\left(\aleph_{0} \odot \boldsymbol{A}\right), \mathfrak{b}\left(\aleph_{0} \odot \boldsymbol{A}\right)^{*}\right) U_{n}^{-1} \rightarrow I$ and $U_{m} p_{k}\left(\mathfrak{b}\left(\aleph_{0} \odot \boldsymbol{B}\right), \mathfrak{b}\left(\aleph_{0} \odot \boldsymbol{B}\right)^{*}\right) U_{m}^{-1} \rightarrow 0$ strongly as $k \rightarrow \infty$. Now if $d$ is a metric on $D=\{T \in \mathcal{B}(\mathcal{K}):\|T\| \leqslant 2\}$ which induces the strong operator topology of $D$, then for every $p \in \mathcal{Q}$ the function $\psi_{p}^{j}: \operatorname{CDD}_{N}\left(\mathcal{H}_{j}\right) \ni \boldsymbol{X} \mapsto U_{j} p\left(\mathfrak{b}\left(\aleph_{0} \odot \boldsymbol{X}\right), \mathfrak{b}\left(\aleph_{0} \odot X\right)^{*}\right) U_{j}^{-1} \in D$ is measurable and thus so is $\theta_{p}: \operatorname{CDD}_{N}\left(\mathcal{H}_{n}\right) \times \operatorname{CDD}_{N}\left(\mathcal{H}_{m}\right) \ni(\boldsymbol{X}, \boldsymbol{Y}) \mapsto$ $d\left(\psi_{p}^{j}(\boldsymbol{X}), I\right)+d\left(\psi_{p}^{m}(\boldsymbol{Y}), 0\right) \in \mathbb{R}_{+}$. Finally, since $\mathcal{Q}$ is countable, also the function $u: \operatorname{CDD}_{N}\left(\mathcal{H}_{n}\right) \times \operatorname{CDD}_{N}\left(\mathcal{H}_{m}\right) \ni(\boldsymbol{X}, \boldsymbol{Y}) \mapsto \inf _{p \in \mathcal{Q}} \theta_{p}(\boldsymbol{X}, \boldsymbol{Y}) \in$ $\mathbb{R}_{+}$is measurable. The observation that $\mathfrak{D}_{N}(n, m)=u^{-1}(\{0\})$ finishes the proof.

19.5. Theorem. The sets

$$
\Delta_{N}(n, m)=\left\{(\boldsymbol{A}, \boldsymbol{B}) \in \mathfrak{F}_{N}\left(\mathcal{H}_{n}\right) \times \mathfrak{F}_{N}\left(\mathcal{H}_{m}\right): \boldsymbol{A} \equiv \boldsymbol{B}\right\}
$$

and $\unlhd_{N}(n, m)=\left\{(\boldsymbol{A}, \boldsymbol{B}) \in \mathfrak{F}_{N}\left(\mathcal{H}_{n}\right) \times \mathfrak{F}_{N}\left(\mathcal{H}_{m}\right): \boldsymbol{A} \leqslant \boldsymbol{B}\right\}$ are measurable.

Proof. First of all, note that for $(\boldsymbol{A}, \boldsymbol{B}) \in \mathfrak{F}_{N}\left(\mathcal{H}_{n}\right) \times \mathfrak{F}_{N}\left(\mathcal{H}_{m}\right), \boldsymbol{A} \not \not_{u}$ $\boldsymbol{B} \Longleftrightarrow \aleph_{0} \odot \mathbb{A}=\aleph_{0} \odot \mathbb{B}$. So, Lemma 19.4 implies that the set $C(n, m)=\left\{(\boldsymbol{A}, \boldsymbol{B}) \in \mathfrak{F}_{N}\left(\mathcal{H}_{n}\right) \times \mathfrak{F}_{N}\left(\mathcal{H}_{m}\right): \aleph_{0} \odot \mathbb{A}=\aleph_{0} \odot \mathbb{B}\right\}$ is measurable. Put $L_{N}(n, m)=\left\{(\boldsymbol{A}, \boldsymbol{B}) \in \mathfrak{F}_{N}\left(\mathcal{H}_{n}\right) \times \mathfrak{F}_{N}\left(\mathcal{H}_{m}\right): \boldsymbol{A} \supsetneqq \boldsymbol{B}\right\}$ and $R_{N}(n, m)=\left\{(\boldsymbol{A}, \boldsymbol{B}):(\boldsymbol{B}, \boldsymbol{A}) \in L_{N}(n, m)\right\}$. Observe that

$$
\unlhd_{N}(n, m)=\Delta_{N}(n, m) \cup L_{N}(n, m),
$$

$C(n, m)=\Delta_{N}(n, m) \cup L_{N}(n, m) \cup R_{N}(n, m)$ and the sets $\Delta_{N}(n, m)$, $L_{N}(n, m)$ and $R_{N}(n, m)$ are pairwise disjoint. Since $C(n, m)$ is a standard Borel space, it suffices therefore to show that each of the latter sets is Souslin (cf. [34, Theorem A.3]). We see that $\Delta(n, m)=\varnothing$ if $n \neq m$ and $\Delta_{N}(n, n)=\left\{\left(\boldsymbol{A}, U \boldsymbol{A} U^{-1}\right): U \in \mathcal{U}\left(\mathcal{H}_{n}\right), \quad \boldsymbol{A} \in \mathfrak{F}_{N}\left(\mathcal{H}_{n}\right)\right\}$ (where $U\left(A_{1}, \ldots, A_{N}\right) U^{-1}=\left(U A_{1} U^{-1}, \ldots, U A_{N} U^{-1}\right)$ ) is the image of a standard Borel space $\mathcal{U}\left(\mathcal{H}_{n}\right) \times \mathfrak{F}_{N}\left(\mathcal{H}_{n}\right)$ under a Borel function and thus $\Delta_{N}(n, n)$ is Souslin. Finally, the set $\mathfrak{F}_{N}^{f i n}\left(\mathcal{H}_{n}\right)$ of all $N$-tuples $\boldsymbol{X} \in \mathfrak{F}_{N}\left(\mathcal{H}_{n}\right)$ such that $\mathcal{W}^{\prime}(\boldsymbol{X})$ is finite is Borel and therefore $L_{N}(n, m)$ is Souslin, since $L(n, m)=\varnothing$ for $n>m$ or $n=m<\infty$, for $n<m$ :

$$
\begin{array}{r}
L_{N}(n, m)=\left\{\left(\boldsymbol{A}, U(\boldsymbol{A} \oplus \boldsymbol{G}) U^{-1}\right): U \in \mathcal{U}\left(\mathcal{H}_{n} \oplus \mathcal{H}_{m-n}, \mathcal{H}_{m}\right),\right. \\
\left.\boldsymbol{A} \in \mathfrak{F}_{N}\left(\mathcal{H}_{n}\right),(\boldsymbol{A}, \boldsymbol{G}) \in C(n, m-n)\right\},
\end{array}
$$


and

$$
\begin{array}{r}
L_{N}(\infty, \infty)=\bigcup_{k=1}^{k=\infty}\left\{\left(\boldsymbol{A}, U(\boldsymbol{A} \oplus \boldsymbol{G}) U^{-1}\right): U \in \mathcal{U}\left(\mathcal{H}_{\infty} \oplus \mathcal{H}_{k}, \mathcal{H}_{\infty}\right),\right. \\
\left.\boldsymbol{A} \in \mathfrak{F}_{N}^{f i n}\left(\mathcal{H}_{\infty}\right),(\boldsymbol{A}, \boldsymbol{G}) \in C(\infty, k)\right\} .
\end{array}
$$

The note that $R_{N}(n, m)$ is the Borel image of $L_{N}(n, m)$ finishes the proof.

19.6. Corollary. Let $\mathcal{F}$ be such a Borel subset of $\mathfrak{F}_{N}\left(\mathcal{H}_{n}\right)$ that the function $\Phi: \mathcal{F} \ni \boldsymbol{X} \mapsto \mathbb{X} \in \mathcal{C D D}_{N}$ is one-to-one. Then $\widehat{\mathcal{F}}=\{\boldsymbol{Y} \in$ $\mathrm{CDD}_{N}\left(\mathcal{H}_{n}\right): \boldsymbol{Y} \equiv \boldsymbol{X}$ for some $\left.\boldsymbol{X} \in \mathcal{F}\right\}$ is a Borel subset of $\mathrm{CDD}_{N}\left(\mathcal{H}_{n}\right)$ and $\mathcal{F}=\{\mathbb{X}: \quad X \in \mathcal{F}\} \subset \mathcal{C D D}_{N}$ is measurable and it is a standard Borel space.

Proof. By Theorem 19.5, the set $\mathcal{D}=\Delta_{N}(n, n) \cap\left(\mathrm{CDD}_{N}\left(\mathcal{H}_{n}\right) \times \mathcal{F}\right)$ is Borel. What is more, it follows from the assumptions that the function $\mathcal{D} \ni(\boldsymbol{A}, \boldsymbol{B}) \mapsto \boldsymbol{A} \in \widehat{\mathcal{F}}$ is a bijection. It is also Borel and thus $\widehat{\mathcal{F}} \in$ $\mathfrak{B}\left(\mathrm{CDD}_{N}\left(\mathcal{H}_{n}\right)\right)$, by [34, Corollary A.7]. Since $\left\{\boldsymbol{X} \in \mathrm{CDD}_{N}\left(\mathcal{H}_{n}\right): \mathbb{X} \in\right.$ $\mathcal{F}\}=\widehat{\mathcal{F}}$, we obtain that $\mathcal{F} \in \mathfrak{B}_{N}$.

It is clear that $\Phi$ is a Borel bijection of $\mathcal{F}$ onto $\mathcal{F}$. However, if $\mathcal{B}$ is a Borel subset of $\mathcal{F}$, then the above argument shows that $\{\mathbb{X}: X \in \mathcal{B}\} \in$ $\mathfrak{B}_{N}$ and hence $\Phi$ is a Borel isomorphism and the assertion follows.

A variation of Theorem 19.5 is contained in

19.7. Lemma. For each $t \in(0, \infty)$ the sets $\Delta_{N}^{t}=\left\{(\boldsymbol{A}, \boldsymbol{B}) \in \mathfrak{s}_{N}\left(\mathcal{H}_{\infty}\right) \times\right.$ $\left.\mathfrak{s}_{N}\left(\mathcal{H}_{\infty}\right): \mathbb{A}=t \odot \mathbb{B}\right\}$ and $\unlhd_{N}^{t}=\left\{(\boldsymbol{A}, \boldsymbol{B}) \in \mathfrak{s}_{N}\left(\mathcal{H}_{\infty}\right) \times \mathfrak{s}_{N}\left(\mathcal{H}_{\infty}\right): \mathbb{A} \leqslant\right.$ $t \odot \mathbb{B}\}$ are measurable.

Proof. Since $\Delta_{N}^{t}=\unlhd_{N}^{t} \cap \unrhd_{N}^{t}$ where $\unrhd_{N}^{t}=\left\{(\boldsymbol{A}, \boldsymbol{B}): \quad(\boldsymbol{B}, \boldsymbol{A}) \in \unlhd_{N}^{t}\right\}$, it is enough to prove that $\unlhd_{N}^{t}$ is measurable. It is clear that for every $n \geqslant 1$ the function $\mathfrak{s}_{N}\left(\mathcal{H}_{\infty}\right) \ni \boldsymbol{A} \mapsto n \odot \boldsymbol{A} \in \mathfrak{s}_{N}\left(n \odot \mathcal{H}_{\infty}\right)$ is measurable. Consequently, thanks to Theorem 19.5, the set $D(n, m)=\{(\boldsymbol{A}, \boldsymbol{B}) \in$ $\left.\mathfrak{s}_{N}\left(\mathcal{H}_{\infty}\right) \times \mathfrak{s}_{N}\left(\mathcal{H}_{\infty}\right): n \odot \mathbb{A} \leqslant m \odot \mathbb{B}\right\}$ is measurable as well. Now if $w_{k}=\frac{m_{k}}{n_{k}}$ are rationals which decrease to $t$ (as $k$ increases to $\infty$ ), then $\unlhd_{N}^{t}=\bigcap_{k=1}^{\infty} D\left(n_{k}, m_{k}\right)$ and we are done.

Whenever $\mathbb{A}, \mathbb{B} \in \mathfrak{s}_{N}$ are such that $\mathbb{A} \ll \mathbb{B}$, there is a unique positive real number denoted by $\mathbb{A}: \mathbb{B}$ such that

$$
\mathbb{A}=(\mathbb{A}: \mathbb{B}) \odot \mathbb{B} \text {. }
$$

Further, we put $\mathbb{O}: \mathbb{X}=0$ and $(\alpha \odot \mathbb{X}): \mathbb{X}=\alpha$ for any $\mathbb{X} \in \mathfrak{F}_{N}$ and $\alpha \in \operatorname{Card}_{\infty}$, and $(n \odot \mathbb{A}):(m \odot \mathbb{A})=n / m$ for every $\mathbb{A} \in \mathfrak{a}_{N}$ and positive integers $n$ and $m$. It is clear that (19-1) is fulfilled whenever $\mathbb{B} \in \mathfrak{F}_{N}^{\text {fin }}:=\mathfrak{F}_{N} \cap \mathcal{F J N}_{N}$ and $\mathbb{A} \in \mathcal{C D} \mathcal{D}_{N}$ are such that $\mathbb{A} \ll \mathbb{B}$.

For $n, m \in\{1,2, \ldots, \infty\}$ put $\nabla_{N}(m, n)=\left\{(\boldsymbol{A}, \boldsymbol{B}) \in \mathfrak{F}_{N}\left(\mathcal{H}_{m}\right) \times\right.$ $\left.\mathfrak{F}_{N}^{f i n}\left(\mathcal{H}_{n}\right): \mathbb{A} \ll \mathbb{B}\right\}$. It follows from Lemma 19.4 that $\nabla_{N}(m, n)$ is a 
Borel subset of $\operatorname{CDD}_{N}\left(\mathcal{H}_{m}\right) \times \operatorname{CDD}_{N}\left(\mathcal{H}_{n}\right)$. What we want is to show measurability of the function

$$
\text { Div : } \nabla_{N}(m, n) \ni(\boldsymbol{X}, \boldsymbol{Y}) \mapsto \mathbb{X}: \mathbb{Y} \in I_{\aleph_{0}} .
$$

It may be easily shown that Div is measurable on $\nabla_{N}(m, n)$ for finite $n$ and on $\nabla_{N}(\infty, \infty) \backslash\left(\mathfrak{s}_{N}\left(\mathcal{H}_{\infty}\right) \times \mathfrak{s}_{N}\left(\mathcal{H}_{\infty}\right)\right)(\nabla(n, \infty)$ is empty if $n$ is finite). On the other hand, $\operatorname{Div}^{-1}((0, t]) \cap\left(\mathfrak{s}_{N}\left(\mathcal{H}_{\infty}\right) \times \mathfrak{s}_{N}\left(\mathcal{H}_{\infty}\right)\right)=\unlhd_{N}^{t}$ and therefore Div is measurable on $\nabla_{N}(\infty, \infty) \cap\left(\mathfrak{s}_{N}\left(\mathcal{H}_{\infty}\right) \times \mathfrak{s}_{N}\left(\mathcal{H}_{\infty}\right)\right)$ as well. As a corollary of this we get that the sets $\{(t, \boldsymbol{A}, \boldsymbol{B}) \in(0, \infty) \times$ $\left.\mathfrak{s}_{N}\left(\mathcal{H}_{\infty}\right) \times \mathfrak{s}_{N}\left(\mathcal{H}_{\infty}\right): \mathbb{A} \leqslant t \odot \mathbb{B}\right\}$ and

$$
\begin{aligned}
& \mathscr{R}_{N}(n, m)=\left\{(\mathbb{A}: \mathbb{B}, \boldsymbol{B}, \boldsymbol{A}) \in I_{\aleph_{0}} \times \mathfrak{F}_{N}^{\text {fin }}\left(\mathcal{H}_{n}\right) \times \mathfrak{F}_{N}\left(\mathcal{H}_{m}\right) \mid\right. \\
&\mathbb{A} \ll \mathbb{B}\} \cup\left\{\left(\aleph_{0}, \boldsymbol{B}, \boldsymbol{B}\right): \boldsymbol{B} \in \mathfrak{F}_{N}\left(\mathcal{H}_{n}\right) \backslash \mathfrak{F}_{N}^{\text {fin }}\left(\mathcal{H}_{n}\right)\right\}
\end{aligned}
$$

are measurable. This fact will be used in the proof of

19.8. Theorem. Let $(X, \mathfrak{M}, \mu)$ be a standard measure space, $\mathcal{F} \subset \mathfrak{F}_{N}$ be a countably separated measurable set and $\Phi: X \ni x \mapsto \mathbb{A}^{(x)} \in \mathcal{F}$ be a measurable function. Further, let $f: X \rightarrow I_{\aleph_{0}} \backslash\{0\}$ be a Borel function such that $f\left(X \backslash \Phi^{-1}\left(\mathfrak{s}_{N}\right)\right) \subset$ Card. Then there are measurable sets $X_{1}, X_{2}, \ldots, X_{\infty} \subset X$ and Borel functions $\Phi_{n}: X_{n} \ni x \mapsto \boldsymbol{B}^{(x)} \in$ $\mathrm{CDD}_{N}\left(\mathcal{H}_{n}\right)(n=1,2, \ldots, \infty)$ such that $\mathbb{B}^{(x)}=f(x) \odot \mathbb{A}^{(x)}$ for each $x \in X^{\prime}:=\bigcup_{n=1}^{n=\infty} X_{n}$ and $\mu\left(X \backslash X^{\prime}\right)=0$. If, in addition,

$$
\mathbb{A}^{(x)} \perp_{u} \mathbb{A}^{(y)}
$$

for distinct $x, y \in X$, then $\Phi_{n}\left(X_{n}\right) \in \mathfrak{B}\left(\operatorname{CDD}_{N}\left(\mathcal{H}_{n}\right)\right)$ and $\Phi_{n}$ is a Borel isomorphism of $X_{n}$ onto its range.

Proof. Since $\Phi^{-1}\left(\mathfrak{F}_{N} \backslash \mathfrak{F}_{N}^{\text {fin }}\right)$ is measurable, we may change the function $f$ (with no change of $f(x) \odot \mathbb{A}^{(x)}$ ) so that $f(x)=\aleph_{0}$ whenever $\Phi(x) \notin$ $\mathfrak{F}_{N}^{f i n}$. But then for every $x \in X$,

$$
\left(f(x) \odot \mathbb{A}^{(x)}\right): \mathbb{A}^{(x)}=f(x) .
$$

Let $\nu: \mathfrak{B}(\mathcal{F}) \ni A \mapsto \mu\left(\Phi^{-1}(A)\right) \in[0, \infty]$. Since $\mathcal{F}$ is the Borel image of a standard Borel space $\bigcup_{n=1}^{n=\infty}\left\{X \in \mathrm{CDD}_{N}\left(\mathcal{H}_{n}\right): \mathbb{X} \in \mathcal{F}\right\}$ (and $\mathcal{F}$ is countably separated), $\mathcal{F}$ is a Souslin-Borel space and therefore $\nu$ is a standard measure on $\mathcal{F}$ (cf. [34, Corollary A.14]). So, we may assume (reducing $\mathcal{F}$ and $X$ ) that $\mathcal{F}$ and $X$ are standard Borel spaces. For each $n=1,2, \ldots, \infty$ let $\mathcal{G}_{n}$ be the set of all $N$-tuples $\boldsymbol{X} \in \mathrm{CDD}_{N}\left(\mathcal{H}_{n}\right)$ such that $\mathbb{X} \in \mathcal{F}(n):=\mathcal{F} \cap \mathcal{S E} \mathcal{P}_{N}(n)$. Note that $\mathcal{G}_{n} \in \mathfrak{B}\left(\operatorname{CDD}_{N}\left(\mathcal{H}_{n}\right)\right)$. Since $\mathcal{F}$ is a standard Borel space, it follows from [34, Theorem A.16] that there are a set $\mathcal{F}_{n} \in \mathfrak{B}(\mathcal{F}(n))$ and a measurable function $\mathcal{F}_{n} \ni \mathbb{X} \mapsto$ $\boldsymbol{G}^{(\mathbb{X})} \in \mathcal{G}_{n}$ such that $\nu\left(\mathcal{F}(n) \backslash \mathcal{F}_{n}\right)=0$ and $\mathbb{G}^{(\mathbb{X})}=\mathbb{X}$ for each $\mathbb{X} \in \mathcal{F}_{n}$. Again, we may assume that $\mathcal{F}=\bigcup_{n=1}^{n=\infty} \mathcal{F}_{n}\left(\right.$ since $\left.\nu\left(\mathcal{F} \backslash \bigcup_{n=1}^{n=\infty} \mathcal{F}_{n}\right)=0\right)$. Put $X(n)=\left\{x \in X: \mathbb{A}^{(x)} \in \mathcal{F}_{n}\right\}$ and $T^{(x)}=G^{\left(\mathbb{A}^{(x)}\right)}$ for $x \in X(n)$. Note that the function $X(n) \ni x \mapsto \boldsymbol{T}^{(x)} \in \mathrm{CDD}_{N}\left(\mathcal{H}_{n}\right)$ is measurable. This implies that the set $\Gamma_{n}=\left\{\left(x, f(x), \boldsymbol{T}^{(x)}\right): x \in X(n)\right\}$ is Borel in 
$X(n) \times I_{\aleph_{0}} \times \operatorname{CDD}_{N}\left(\mathcal{H}_{n}\right)$ (as the graph of a Borel function) and consequently for each $m=1,2, \ldots, \infty$ the set $\mathscr{B}_{n, n m}=\{(x, \boldsymbol{Y}): \mathbb{Y}=$ $\left.f(x) \odot \mathbb{A}^{(x)}, x \in X(n), \quad \boldsymbol{Y} \in \mathfrak{F}_{N}\left(\mathcal{H}_{n m}\right)\right\}$, as the image of $\left(\Gamma_{n} \times\right.$ $\left.\mathfrak{F}_{N}\left(\mathcal{H}_{n m}\right)\right) \cap\left(X(n) \times \mathscr{R}_{N}(n, n m)\right)$ under the projection map (cf. (19-2) and (19-4)) which is one-to-one on the latter set, is Borel as well. Now put $X(n, n m)=\left\{x \in X(n): f(x) \cdot \operatorname{dim}\left(\mathbb{A}^{(x)}\right)=n m\right\}$ and note that $X(n, n m)$ 's are measurable sets such that $X(n)=\bigcup_{m=1}^{m=\infty} X(n, n m)$. Since the function $p_{n, n m}: \mathscr{B}_{n, n m} \ni(x, \boldsymbol{Y}) \mapsto x \in X(n, n m)$ is a Borel surjection, we deduce from [34, Theorem A.16] that there is a Borel function $w_{n, n m}: X(n, n m) \rightarrow \mathscr{B}_{n, n m}$ such that $\left(p_{n, n m} \circ w_{n, n m}\right)(x)=$ $x$ for $\mu$-almost all $x \in X(n, n m)$. For $x \in X(n, n m)$ let $\boldsymbol{B}^{(x)} \in$ $\mathfrak{F}_{N}\left(\mathcal{H}_{n m}\right)$ be the second coordinate of $w_{n, n m}(x)$. Then the function $\Phi_{n, n m}: X(n, n m) \ni x \mapsto \boldsymbol{B}^{(x)} \in \mathrm{CDD}_{N}\left(\mathcal{H}_{n m}\right)$ is measurable and for $\mu$-almost all $x \in X$,

$$
\mathbb{B}^{(x)}=f(x) \odot \mathbb{A}^{(x)} .
$$

Again, by reducing $X$, we may assume that (19-5) is fulfilled for all $x \in X$. To this end, put $X_{k}=\bigcup\{X(n, n m): n m=k\}$ and let $\Phi_{k}: X_{k} \rightarrow \mathrm{CDD}_{N}\left(\mathcal{H}_{k}\right)$ be given by $\Phi_{k}(x)=\Phi_{n, n m}(x)$ provided $n m=k$ and $x \in X(n, n m)$. Since the sets $X(n, n m)$ are pairwise disjoint, $\Phi_{k}$ is well defined and Borel. Finally, if (19-3) is fulfilled, (19-5) implies that $\Phi_{k}$ is one-to-one and thus the assertion follows.

\section{DiRECT integrals AND MEASURABle DOMAins OF STRONG UNITARY DISJOINTNESS}

In this part we establish only the most relevant (for our further investigations) properties of direct integrals. The 'continuous' operation in $\mathcal{C} \mathcal{D} \mathcal{D}_{N}$ is defined and main results on it are placed in the next two sections.

We now fix a standard measure space $(X, \mathfrak{M}, \mu)$. For a separable Hilbert space $\mathcal{H}$ the Hilbert space $L^{2}(X, \mathcal{H})=L^{2}(\mu, \mathcal{H})$ consists of all (equivalence classes of) measurable functions $\xi: X \rightarrow \mathcal{H}$ such that $\|\xi\|_{2}^{2}=\int_{X}\|\xi(x)\|^{2} \mathrm{~d} \mu(x)<\infty\left(L^{2}(\mu, \mathcal{H})\right.$ is separable). Let $X \ni x \mapsto$ $T_{x} \in \operatorname{CDD}(\mathcal{H})$ be a measurable function. We define an operator $T:=$ $\int_{X}^{\oplus} T_{x} \mathrm{~d} \mu(x)$ in $L^{2}(\mu, \mathcal{H})$ by

$$
\begin{aligned}
& \mathcal{D}(T)=\left\{\xi \in L^{2}(\mu, \mathcal{H}): \xi(x) \in \mathcal{D}\left(T_{x}\right) \text { for } \mu \text {-almost all } x \in X\right. \\
&\text { and } \left.\int_{X}\left\|T_{x} \xi(x)\right\|^{2} \mathrm{~d} \mu(x)<\infty\right\}
\end{aligned}
$$

and $(T \xi)(x)=T_{x} \xi(x)$ for $\xi \in \mathcal{D}(T)$ and ( $\mu$-almost all) $x \in X$. It is not obvious that $T \xi$ is measurable (for $\xi \in \mathcal{D}(T)$ ) and that $T \in \operatorname{CDD}(\mathcal{H})$. These are guaranteed by the next result which may be deduced from [35, Lemma VI.3.3] (cf. [35, Definition VI.3.4]). 
20.1. Proposition. For every measurable function $X \ni x \mapsto T_{x} \in$ $\operatorname{CDD}(\mathcal{H})$ the operator $\int_{X}^{\oplus} T_{x} \mathrm{~d} \mu(x)$ is well defined, closed and densely defined. What is more, $\mathfrak{b}\left(\int_{X}^{\oplus} T_{x} \mathrm{~d} \mu(x)\right)=\int_{X}^{\oplus} \mathfrak{b}\left(T_{x}\right) \mathrm{d} \mu(x)$.

Now let $\Phi: X^{\prime} \ni x \mapsto T^{(x)} \in \bigcup_{n=1}^{n=\infty} \operatorname{CDD}_{N}\left(\mathcal{H}_{n}\right)$ where $X \backslash X^{\prime} \in$ $\mathcal{N}(\mu)$ be any function and $\boldsymbol{T}^{(x)}=\left(\boldsymbol{T}_{1}^{(x)}, \ldots, \boldsymbol{T}_{N}^{(x)}\right)$ for each $x \in X^{\prime}$. If there are measurable sets $X_{1}, X_{2}, \ldots, X_{\infty} \subset X^{\prime}$ such that $\mu\left(X^{\prime}\right)$ $\left.\bigcup_{n=1}^{n=\infty} X_{n}\right)=0, \Phi\left(X_{j}\right) \subset \operatorname{CDD}_{N}\left(\mathcal{H}_{j}\right)$ (the latter implies that $X_{j}$ 's are pairwise disjoint) and $\left.\Phi\right|_{X_{j}}: X_{j} \rightarrow \operatorname{CDD}_{N}\left(\mathcal{H}_{j}\right)$ is measurable for each $j$, we call $\Phi$ integrable and define the direct integral $\int_{X}^{\oplus} \boldsymbol{T}^{(x)} \mathrm{d} \mu(x)$ of the field $\left\{\boldsymbol{T}^{(x)}\right\}_{x \in X^{\prime}}$ by

$$
\int_{X}^{\oplus} \boldsymbol{T}^{(x)} \mathrm{d} \mu(x)=\bigoplus_{n=1}^{n=\infty}\left(\int_{X_{n}}^{\oplus} T_{1}^{(x)} \mathrm{d} \mu(x), \ldots, \int_{X_{n}}^{\oplus} T_{N}^{(x)} \mathrm{d} \mu(x)\right) .
$$

Below we list most important (for our investigations) properties of direct integrals of measurable fields of $N$-tuples.

(di0) $\operatorname{dim} \overline{\mathcal{D}}\left(\int_{X}^{\oplus} \boldsymbol{T}^{(x)} \mathrm{d} \mu(x)\right) \leqslant \aleph_{0}$.

(di1) $\mathfrak{b}\left(\int_{X}^{\oplus} \boldsymbol{T}^{(x)} \mathrm{d} \mu(x)\right)=\int_{X}^{\oplus} \mathfrak{b}\left(\boldsymbol{T}^{(x)}\right) \mathrm{d} \mu(x)$.

(di2) If $X_{1}, X_{2}, X_{3}, \ldots$ are pairwise disjoint measurable subsets of $X$ such that $\mu\left(X_{j}\right)>0$ for each $j$ and $\mu\left(X \backslash \bigcup_{n=1}^{\infty} X_{n}\right)=0$, then

$$
\int_{X}^{\oplus} \boldsymbol{A}^{(x)} \mathrm{d} \mu(x) \equiv \bigoplus_{n=1}^{\infty} \int_{X_{n}}^{\oplus} \boldsymbol{A}^{(x)} \mathrm{d} \mu(x)
$$

(di3) $\bigoplus_{n=1}^{\infty}\left(\int_{X}^{\oplus} \boldsymbol{T}_{n}^{(x)} \mathrm{d} \mu(x)\right) \equiv \int_{X}^{\oplus}\left(\bigoplus_{n=1}^{\infty} \boldsymbol{T}_{n}^{(x)}\right) \mathrm{d} \mu(x)$.

(di4) If $\boldsymbol{T}^{(x)} \equiv \boldsymbol{S}^{(x)}$ for $\mu$-almost all $x \in X$, then $\int_{X}^{\oplus} \boldsymbol{T}^{(x)} \mathrm{d} \mu(x) \equiv$ $\int_{X}^{\oplus} S^{(x)} \mathrm{d} \mu(x)$. This follows from (BT5) (page 8), (di1) and the proof of [34, Theorem IV.8.28].

(di5) If $\nu$ is a $\sigma$-finite measure on $(X, \mathfrak{M})$ such that $\nu \ll \mu \ll \nu$ (that is, $\mathcal{N}(\mu)=\mathcal{N}(\nu))$, then $\int_{X}^{\oplus} \boldsymbol{T}^{(x)} \mathrm{d} \mu(x) \equiv \int_{X}^{\oplus} \boldsymbol{T}^{(x)} \mathrm{d} \nu(x)$.

(di6) If $(Y, \mathfrak{N}, \nu)$ is a standard measure space, $X_{0} \in \mathcal{N}(\mu), Y_{0} \in \mathcal{N}(\nu)$ and $\psi: Y \backslash Y_{0} \rightarrow X \backslash X_{0}$ is a Borel isomorphism such that $\mu(\psi(A))=\nu(A)$ for every $A \in \mathfrak{N}$ disjoint from $Y_{0}$, then

$$
\int_{X}^{\oplus} \boldsymbol{T}^{(x)} \mathrm{d} \mu(x) \equiv \int_{Y}^{\oplus} \boldsymbol{T}^{(\psi(y))} \mathrm{d} \nu(y) .
$$

Further, let $X \ni x \mapsto \mathbb{A}^{(x)} \in \mathcal{S} \mathcal{E} \mathcal{P}_{N}$ be any function. If there exist sets $X_{1}, X_{2}, \ldots, X_{\infty} \in \mathfrak{M}$ and measurable functions

$$
X_{n} \ni x \mapsto \boldsymbol{A}^{(x)} \in \mathrm{CDD}_{N}\left(\mathcal{H}_{n}\right)
$$

$(n=1,2, \ldots, \infty)$ such that $\mu\left(X \backslash \bigcup_{n=1}^{n=\infty} X_{n}\right)=0$ and for each $x \in$ $\bigcup_{n=1}^{n=\infty} X_{n}, A^{(x)}$ is a representative of $\mathbb{A}^{(x)}$, we say the field $\left\{\mathbb{A}^{(x)}\right\}_{x \in X}$ is integrable and define the direct integral $\int_{X}^{\oplus} \mathbb{A}^{(x)} \mathrm{d} \mu(x)$ as the unitary 
equivalence class of

$$
\bigoplus_{n=1}^{n=\infty} \int_{X_{n}}^{\oplus} A^{(x)} \mathrm{d} \mu(x) .
$$

Thanks to (di4), $\int_{X}^{\oplus} \mathbb{A}^{(x)} \mathrm{d} \mu(x)$ is well defined, i.e. it does not depend of the choice of measurable functions (20-1) of representatives. As it is easily seen, in the above situation the function $\bigcup_{n=1}^{n=\infty} X_{n} \ni x \mapsto \mathbb{A}^{(x)} \in$ $\mathcal{S} \mathcal{E} \mathcal{P}_{N}$ is measurable. We call a field $\Psi: X \ni x \mapsto \mathbb{B}^{(x)} \in \mathcal{S} \mathcal{E} \mathcal{P}_{N}$ almost measurable (or almost Borel) iff $\left.\Psi\right|_{X \backslash X_{0}}$ is Borel for some $X_{0} \in \mathcal{N}(\mu)$. Thus, every integrable field is almost measurable.

In our investigations all almost measurable fields are defined on standard measure spaces. Properties (di0)-(di6) may naturally be translated into the realm of unitary equivalence classes of $N$-tuples:

(DI0) $\int_{X}^{\oplus} \mathbb{A}^{(x)} \mathrm{d} \mu(x) \in \mathcal{S E} \mathcal{P}_{N}$.

(DI1) $\mathfrak{b}\left(\int_{X}^{\oplus} \mathbb{A}^{(x)} \mathrm{d} \mu(x)\right)=\int_{X}^{\oplus} \mathfrak{b}\left(\mathbb{A}^{(x)}\right) \mathrm{d} \mu(x)$.

(DI2) If $X_{1}, X_{2}, X_{3}, \ldots$ are pairwise disjoint measurable subsets of $X$ such that $\mu\left(X_{j}\right)>0$ for each $j$ and $\mu\left(X \backslash \bigcup_{n=1}^{\infty} X_{n}\right)=0$, then

$$
\int_{X}^{\oplus} \mathbb{A}^{(x)} \mathrm{d} \mu(x)=\bigoplus_{n=1}^{\infty} \int_{X_{n}}^{\oplus} \mathbb{A}^{(x)} \mathrm{d} \mu(x) .
$$

(DI3) $\bigoplus_{n=1}^{\infty}\left(\int_{X}^{\oplus} \mathbb{T}_{n}^{(x)} \mathrm{d} \mu(x)\right)=\int_{X}^{\oplus}\left(\bigoplus_{n=1}^{\infty} \mathbb{T}_{n}^{(x)}\right) \mathrm{d} \mu(x)$.

(DI4) If $(Y, \mathfrak{N}, \nu)$ is a standard measure space, $X_{0} \in \mathcal{N}(\mu), Y_{0} \in \mathcal{N}(\nu)$, $\psi: Y \backslash Y_{0} \rightarrow X \backslash X_{0}$ is a Borel isomorphism and $\{\psi(B): B \in$ $\left.\mathcal{N}(\nu), B \cap Y_{0}=\varnothing\right\}=\left\{A \in \mathcal{N}(\nu): A \cap X_{0}=\varnothing\right\}$, then

$$
\int_{X}^{\oplus} \mathbb{A}^{(x)} \mathrm{d} \mu(x)=\int_{Y}^{\oplus} \mathbb{A}^{(\psi(y))} \mathrm{d} \nu(y) .
$$

A counterpart of regular collections and direct sums ((UE4), page 7) for direct integrals are regular fields and regular direct integrals ' $\int^{\boxplus \text {, }}$ which we define as follows. Assume $X \ni x \mapsto \mathbb{A}^{(x)} \in \mathcal{S} \mathcal{E} \mathcal{P}_{N}$ is an integrable field. If for any two disjoint Borel sets $A, B \subset X$ one has

$$
\int_{A}^{\oplus} \mathbb{A}^{(x)} \mathrm{d} \mu(x) \perp_{u} \int_{B}^{\oplus} \mathbb{A}^{(x)} \mathrm{d} \mu(x),
$$

we call the field $\left\{\mathbb{A}^{(x)}\right\}_{x \in X}$ regular and write $\int_{X}^{\boxplus} \mathbb{A}^{(x)} \mathrm{d} \mu(x)$ in place of $\int_{X}^{\oplus} \mathbb{A}^{(x)} \mathrm{d} \mu(x)$. (Condition (20-3) naturally corresponds to (PR2),

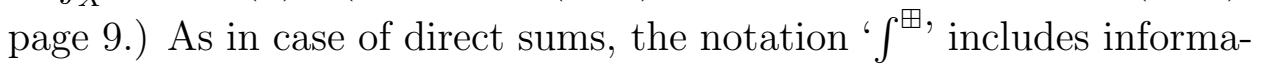
tion that the integrable field is regular.

In practice it is quite difficult to verify whether an almost measurable field is integrable. However, as an immediate consequence of Proposition 19.3 we obtain

20.2. Proposition. Every almost measurable field of a standard measure space into $\mathcal{S} \mathcal{E} \mathcal{P}_{N} \backslash \mathcal{S} \mathcal{E} \mathcal{P}_{N}(\infty)$ is integrable. 
Proof. Let $\Phi: X \rightarrow \mathcal{S} \mathcal{E} \mathcal{P}_{N} \backslash \mathcal{S} \mathcal{E} \mathcal{P}_{N}(\infty)$ be measurable. The sets $X_{n}=$ $\Phi^{-1}\left(\mathcal{S} \mathcal{E} \mathcal{P}_{N}(n)\right)$ are Borel and if $\chi_{n}$ 's are as in Proposition 19.3 (page 70), then $\left.\chi_{n} \circ \Phi\right|_{X_{n}}$ is a measurable field of representatives for $\Phi$.

In general we are unable to characterize integrable fields taking values in $\mathcal{S} \mathcal{E} \mathcal{P}_{N}$. This is in fact not of our interest. More preferable are regular fields taking values in $\mathfrak{F}_{N}$. In that case a characterization is possible and we formulate it in the next result. For this purpose we introduce

20.3. Definition. A set $\mathcal{F} \in \mathfrak{B}_{N}$ is said to be a measurable domain of strong unitary disjointness iff there is a sequence $\left(\mathcal{E}_{n}\right)_{n=1}^{\infty}$ of subsets of $\operatorname{CDD}_{N}$ which separates points of $\mathcal{F}$ and for every $n \geqslant 1$ the families $\mathcal{F} \cap \mathcal{E}_{n}$ and $\mathcal{F} \backslash \mathcal{E}_{n}$ are strongly unitarily disjoint (cf. Remark 18.5, page 66). We shall shorten the name of this and we shall speak briefly of measurable domains.

It follows from the definition that measurable domains consist of pairwise unitarily disjoint $N$-tuples. It may also be easily verified that the union of a countable family of measurable domains each two of which are strongly unitarily disjoint as well as every measurable subset of a measurable domain is again a measurable domain. Another important property of measurable domains is that they are Souslin-Borel. Indeed, when $\mathcal{F}$ is a measurable domain, it is the Borel image of a standard Borel space (by measurability of $\mathcal{F}$ ) and $\mathcal{F}$ is countably separated, since if $\mathcal{E} \subset \mathcal{C D D} \mathcal{D}_{N}$ is such that $\mathcal{F} \cap \mathcal{E} \perp_{s} \mathcal{F} \backslash \mathcal{E}$, then $\mathcal{F} \cap \mathcal{E} \in \mathfrak{B}_{N}$ (because for every sequence $\left(p_{n}\right)_{n=1}^{\infty} \subset \mathcal{P}_{1}(N)$ and each complex scalar $\lambda$ the set of all $\boldsymbol{T} \in \mathrm{CDD}_{N}\left(\mathcal{H}_{k}\right)$ such that $p_{n}\left(\mathfrak{b}(\boldsymbol{T}), \mathfrak{b}(\boldsymbol{T})^{*}\right)$ converges $*$-strongly to $\lambda I$ is Borel and invariant under unitary equivalence), and thus the assertion follows from Definition 20.3.

Measurable domains are useful to produce regular fields, as it is shown by

20.4. Proposition. Let $(X, \mathfrak{M}, \mu)$ be a standard measure space and $\Phi: X \ni x \mapsto \mathbb{A}^{(x)} \in \mathfrak{F}_{N}$ be any field. Then the following conditions are equivalent:

(i) $\left\{\mathbb{A}^{(x)}\right\}_{x \in X}$ is regular,

(ii) there is a Borel set $X^{\prime} \subset X$ such that $X \backslash X^{\prime} \in \mathcal{N}(\mu), \Phi\left(X^{\prime}\right)$ is a measurable domain and $\left.\Phi\right|_{X^{\prime}}$ is a Borel isomorphism of $X^{\prime}$ onto its range.

Proof. First of all, by reducing $X$, we may assume that $X$ is a standard Borel space. Suppose condition (i) is satisfied. This yields that there is $Z \in \mathcal{N}(\mu)$ and an integrable field $\left\{\boldsymbol{A}^{(x)}\right\}_{x \in X \backslash Z} \subset \bigcup_{n=1}^{n=\infty} \mathrm{CDD}_{N}\left(\mathcal{H}_{n}\right)$ of representatives for $\Phi$. Take a separating sequence $X_{1}, X_{2}, \ldots$ of measurable subsets of $X$. We infer from (di0), (20-3) and Proposition 18.3 (page 65) that for each $k \geqslant 1$ there is a sequence $\left(q_{n}^{(k)}\right)_{n=1}^{\infty} \subset \mathcal{P}_{1}(N)$ 
such that

$$
\begin{gathered}
q_{n}^{(k)}\left(\mathfrak{b}\left(\int_{X_{k}}^{\oplus} \boldsymbol{A}^{(x)} \mathrm{d} \mu(x)\right), \mathfrak{b}\left(\int_{X_{k}}^{\oplus} \boldsymbol{A}^{(x)} \mathrm{d} \mu(x)\right)^{*}\right) \stackrel{* s}{\rightarrow} I, \\
q_{n}^{(k)}\left(\mathfrak{b}\left(\int_{X \backslash X_{k}}^{\oplus} \boldsymbol{A}^{(x)} \mathrm{d} \mu(x)\right), \mathfrak{b}\left(\int_{X \backslash X_{k}}^{\oplus} \boldsymbol{A}^{(x)} \mathrm{d} \mu(x)\right)^{*}\right) \stackrel{* s}{\rightarrow} 0 .
\end{gathered}
$$

Now taking into account that

$$
\begin{aligned}
p\left(\mathfrak{b}\left(\int_{D}^{\oplus} A^{(x)} \mathrm{d} \mu(x)\right), \mathfrak{b}\left(\int_{D}^{\oplus} A^{(x)} \mathrm{d} \mu(x)\right)^{*}\right)= \\
\quad=\int_{D}^{\oplus} p\left(\mathfrak{b}\left(\boldsymbol{A}^{(x)}\right), \mathfrak{b}\left(\boldsymbol{A}^{(x)}\right)^{*}\right) \mathrm{d} \mu(x)
\end{aligned}
$$

for every measurable set $D \subset X$ and $p \in \mathcal{P}(N)$ (cf. (di1)), we infer from [29, Proposition 3.2.7] that there are a subsequence $\left(p_{n}^{(k)}\right)_{n=1}^{\infty}$ of $\left(q_{n}^{(k)}\right)_{n=1}^{\infty}$ and a measurable set $X_{k}^{\prime} \subset X \backslash Z$ such that $X \backslash X_{k}^{\prime} \in \mathcal{N}(\mu)$ and

$$
p\left(\mathfrak{b}\left(\boldsymbol{A}^{(x)}\right), \mathfrak{b}\left(\boldsymbol{A}^{(x)}\right)^{*}\right) \stackrel{* s}{\rightarrow} j_{k}(x) I
$$

for any $x \in X_{k}^{\prime}$ where $j_{k}$ is the characteristic function of $X_{k}$. Put $X^{\prime}=\bigcap_{k=1}^{\infty} X_{k}^{\prime}$ and note that $\mu\left(X \backslash X^{\prime}\right)=0$. Since $\left\{X_{k}\right\}_{k \geqslant 1}$ is a separating family and thanks to $(20-5),\left.\Phi\right|_{X^{\prime}}$ is one-to-one. It may be also deduced from Corollary 19.6 (page 73 ) that $\Phi\left(X^{\prime}\right)$ is measurable. Consequently, $\Phi\left(X^{\prime}\right)$ is a measurable domain, by (20-5). Now it suffices to apply [34, Corollary A.10] to get that $\left.\Phi\right|_{X^{\prime}}$ is a Borel isomorphism.

We now pass to the converse implication. It follows from Theorem 19.8 (page 74) that $\Phi$ is integrable. So, let

$$
\left\{A^{(x)}\right\}_{x \in X^{\prime \prime}} \subset \bigcup_{n=1}^{n=\infty} \operatorname{CDD}_{N}\left(\mathcal{H}_{n}\right)
$$

be an integrable field of representatives for $\Phi$ where $X^{\prime \prime} \subset X^{\prime}$ and $X \backslash X^{\prime \prime} \in \mathcal{N}(\mu)$. Put $\boldsymbol{A}=\int_{X}^{\oplus} \boldsymbol{A}^{(x)} \mathrm{d} \mu(x)$. Let $\mathcal{E}_{1}, \mathcal{E}_{2}, \ldots$ be a separating family for $\Phi\left(X^{\prime}\right)$ such that

$$
\Phi\left(X^{\prime}\right) \cap \mathcal{E}_{k} \perp_{s} \Phi\left(X^{\prime}\right) \backslash \mathcal{E}_{k}
$$

for every $k$. It follows from the note preceding the proposition that $\mathcal{E}_{k} \cap \Phi\left(X^{\prime}\right) \in \mathfrak{B}_{N}$. Consequently, the sets $X_{k}=\Phi^{-1}\left(\mathcal{E}_{k}\right) \cap X^{\prime \prime}(k=$ $1,2, \ldots)$ are measurable and separate the points of $X^{\prime \prime}$ (because $\Phi$ is one-to-one on $\left.X^{\prime} \supset X^{\prime \prime}\right)$. We infer from this, thanks to [34, Corollary A.12], that the $\sigma$-algebra of subsets of $X^{\prime \prime}$ generated by the sets $X_{k}$ 's coincides with $\mathfrak{M}^{\prime \prime}:=\left\{A \subset X^{\prime \prime} \mid \quad A \in \mathfrak{M}\right\}$. Further, the space $\overline{\mathcal{D}}(A)$ has the form $\bigoplus_{n=1}^{n=\infty} L^{2}\left(X_{n}^{\prime \prime}, \mathcal{H}_{n}\right)$ where $X_{1}^{\prime \prime}, X_{2}^{\prime \prime}, \ldots$ are pairwise disjoint members of $\mathfrak{M}^{\prime \prime}$ whose union is $X^{\prime \prime}$. For each $k$ let $M_{k}$ be the multiplication operator by the characteristic function $j_{k}$ of $X_{k}^{\prime \prime}$ on $\overline{\mathcal{D}}(A)$. Fix for a moment $k$. By (20-6), there is a sequence $\left(p_{n}\right)_{n=1}^{\infty} \subset \mathcal{P}_{1}(N)$ 
such that $p_{n}\left(\mathfrak{b}\left(\boldsymbol{A}^{(x)}\right), \mathfrak{b}\left(\boldsymbol{A}^{(x)}\right)^{*}\right)$ converges $*$-strongly to $j_{k}(x) I$ for every $x \in X^{\prime \prime}$. Since in addition $\left\|p_{n}\left(\mathfrak{b}\left(\boldsymbol{A}^{(x)}\right), \mathfrak{b}\left(\boldsymbol{A}^{(x)}\right)^{*}\right)\right\| \leqslant 1$, Proposition 3.2.7 of [29] implies that

$$
\int_{X^{\prime \prime}}^{\oplus} p_{n}\left(\mathfrak{b}\left(\boldsymbol{A}^{(x)}\right), \mathfrak{b}\left(\boldsymbol{A}^{(x)}\right)^{*}\right) \mathrm{d} \mu(x) \stackrel{* s}{\rightarrow} \int_{X^{\prime \prime}}^{\oplus} j_{k}(x) I \mathrm{~d} \mu(x) .
$$

This combined with $(20-4)$ gives $p_{n}\left(\mathfrak{b}(\boldsymbol{A}), \mathfrak{b}(\boldsymbol{A})^{*}\right) \stackrel{* s}{\rightarrow} M_{k}$ and consequently $M_{k} \in \mathcal{W}^{\prime \prime}(A)$. In this way we have shown that $\left\{X_{1}, X_{2}, \ldots\right\} \subset$ $\mathfrak{N}$ where $\mathfrak{N}$ consists of all $B \in \mathfrak{M}^{\prime \prime}$ such that the multiplication operator $M(B)$ by the characteristic function of $B$ belongs to $\mathcal{W}^{\prime \prime}(A)$. Since $\mathfrak{N}$ is a $\sigma$-algebra, we finally obtain that $\mathfrak{N}=\mathfrak{M}^{\prime \prime}$.

Since $\mathcal{W}^{\prime \prime}(A)=\mathcal{W}(\mathfrak{b}(A))$ and each entry of $\mathfrak{b}(A)$ is a decomposable operator, $\mathcal{W}^{\prime \prime}(A)$ consists of decomposable operators. If $B$ is an arbitrary member of $\mathfrak{M}, M\left(B \cap X^{\prime \prime}\right)$ is a diagonalizable operator and hence $M\left(B \cap X^{\prime \prime}\right) \in \mathcal{Z}\left(\mathcal{W}^{\prime \prime}(A)\right)$. So, $\int_{B}^{\oplus} \mathbb{A}^{(x)} \mathrm{d} \mu(x)\left(=\int_{B \cap X^{\prime \prime}}^{\oplus} \mathbb{A}^{(x)} \mathrm{d} \mu(x)\right)$ and $\int_{X \backslash B}^{\oplus} \mathbb{A}^{(x)} \mathrm{d} \mu(x)$ correspond (by Proposition 4.1, page 9 ) to mutually orthogonal central projections in $\mathcal{W}^{\prime \prime}(A)$ from which we conclude that $\int_{B}^{\oplus} \mathbb{A}^{(x)} \mathrm{d} \mu(x) \perp_{u} \int_{X \backslash B}^{\oplus} \mathbb{A}^{(x)} \mathrm{d} \mu(x)$. Now (20-3) is implied by (di2).

20.5. Remark. Since every Borel injection of a standard Borel space into a Souslin-Borel one has measurable image and is a Borel isomorphism between its domain and range (cf. Theorem A.6 and Corollary A.7 in [34]), condition (ii) of Proposition 20.4 may be weakened by replacing the assumption that $\Phi\left(X^{\prime}\right)$ is a measurable domain and $\left.\Phi\right|_{X^{\prime}}$ is a Borel isomorphism by the one that $\left.\Phi\right|_{X^{\prime}}$ is Borel and one-to-one and $\Phi\left(X^{\prime}\right)$ is contained in a measurable domain.

For simplicity, let us call a $\sigma$-finite measure $\nu$ on a measurable set $\mathcal{B} \subset \mathfrak{F}_{N}$ a regularity measure $(\nu \in \operatorname{rgm}(\mathcal{B}))$ if $\nu$ is standard and the identity field of $\mathcal{B}$ into $\mathfrak{F}_{N}$ is regular. Equivalently, $\nu \in \operatorname{rgm}(\mathcal{B})$ iff $\nu$ is concentrated on a measurable domain (since measurable domains are Souslin-Borel and all $\sigma$-finite measures on the latter sets are standard). To shorten statements, we shall write $(\mu, \Phi) \in \operatorname{RGS}(X, \mathfrak{M})$ to express that $\mu$ is a standard measure on $(X, \mathfrak{M})$ and $\Phi: X \rightarrow \mathfrak{F}_{N}$ is a regular field.

Suppose $(\mu, \Phi) \in \operatorname{RGS}(X, \mathfrak{M})$ is a regular field. Let $X^{\prime}$ be as in point (ii) of Proposition 20.4. Define a measure $\nu=\Phi^{*}(\mu): \mathfrak{B}\left(\mathfrak{F}_{N}\right) \rightarrow$ $[0, \infty]$ by $\nu(\mathcal{B})=\mu\left(\Phi^{-1}(\mathcal{B}) \cap X^{\prime}\right)$. Notice that $\nu \in \operatorname{rgm}\left(\mathfrak{F}_{N}\right)$ and that $\int_{X}^{\boxplus} \Phi(x) \mathrm{d} \mu(x)=\int_{\mathfrak{F}_{N}}^{\boxplus} \mathbb{F} \mathrm{d} \nu(\mathbb{F})$, thanks to (DI4). This observation shows that it suffices to consider regularity measures instead of abstract regular fields.

The following result is a link between regular fields and central decompositions of von Neumann algebras. 
20.6. Proposition. Let $(X, \mathfrak{M}, \mu)$ be a standard measure space, $\Phi: X \ni$ $x \mapsto A^{(x)} \in \bigcup_{n=1}^{n=\infty} \mathfrak{F}_{N}\left(\mathcal{H}_{n}\right)$ an integrable field and let

$$
\boldsymbol{A}=\int_{X}^{\oplus} \boldsymbol{A}^{(x)} \mathrm{d} \mu(x) .
$$

Then the following conditions are equivalent:

(i) $\left\{\mathbb{A}^{(x)}\right\}_{x \in X}$ is regular,

(ii) $\left\{\mathbb{X} \in \mathcal{C D D}_{N}: \mathbb{X} \leqslant^{s} \mathbb{A}\right\}=\left\{\int_{B}^{\oplus} \mathbb{A}^{(x)} \mathrm{d} \mu(x): B \in \mathfrak{M}\right\}$,

(iii) $\int_{X}^{\oplus} \mathcal{W}^{\prime \prime}\left(A^{(x)}\right) \mathrm{d} \mu(x)$ is the central decomposition of the von Neumann algebra $\mathcal{W}^{\prime \prime}(A)$.

Proof. First of all, note that the field $\left\{\mathcal{W}^{\prime \prime}\left(\boldsymbol{A}^{(x)}\right)\right\}_{x \in X}$ is measurable according to [29, Definition 3.2.9], since $\mathcal{W}^{\prime \prime}\left(A^{(x)}\right)=\mathcal{W}\left(\mathfrak{b}\left(A^{(x)}\right)\right)$. Further, under the assumptions of the proposition, (iii) is equivalent to

(iii') the von Neumann algebra $\mathcal{A}$ of all diagonalizable operators is contained in $\mathcal{W}^{\prime \prime}(A)$.

It is clear that (iii') is implied by (iii). Conversely, when (iii') is fulfilled, $\mathcal{W}^{\prime}(\boldsymbol{A})$ consists of (some) decomposable operators (thanks to [34, Corollary IV.8.16] or [19, Theorem 14.1.10]). We see that so does $\mathcal{W}^{\prime \prime}(\boldsymbol{A})$ (since $\mathfrak{b}(\boldsymbol{A})$ is an $N$-tuple of decomposable operators) and hence $\mathcal{A} \subset \mathcal{W}^{\prime}(\boldsymbol{A})$. This yields that $\mathcal{A} \subset \mathcal{Z}\left(\mathcal{W}^{\prime \prime}(\boldsymbol{A})\right)$. Now using the terminology of Kadison and Ringrose [19], we conclude that $\mathcal{W}^{\prime \prime}(\boldsymbol{A})$ is decomposable (Theorem 14.1.16 and Proposition 14.1.18 in $[19])$, i.e. $\mathcal{W}^{\prime \prime}(A)=\int_{X}^{\oplus} \mathcal{M}_{x} \mathrm{~d} \mu(x)$ for some measurable field $\left\{\mathcal{M}_{x}\right\}_{x \in X}$ of von Neumann algebras. By the uniqueness of the decomposition $\mathfrak{b}(A)=\int_{X}^{\oplus} \mathfrak{b}\left(A^{(x)}\right) \mathrm{d} \mu(x)$ (cf. (di1), page 76), $\mathcal{W}\left(\mathfrak{b}\left(A^{(x)}\right)\right) \subset \mathcal{M}_{x}$ for $\mu$ almost all $x \in X$ and thus $\int_{X}^{\oplus} \mathcal{W}^{\prime \prime}\left(A^{(x)}\right) \mathrm{d} \mu(x) \subset \mathcal{W}^{\prime \prime}(A)$. Since the converse inclusion is immediate, we get $\mathcal{W}^{\prime \prime}(A)=\int_{X}^{\oplus} \mathcal{W}^{\prime \prime}\left(A^{(x)}\right) \mathrm{d} \mu(x)$. This proves (iii) because $\mathcal{W}^{\prime \prime}\left(A^{(x)}\right)$ is a factor for all $x \in X$ and consequently (by [34, Corollary IV.8.20]) $\mathcal{Z}\left(\mathcal{W}^{\prime \prime}(\boldsymbol{A})\right)=\int_{X}^{\oplus} \mathcal{Z}\left(\mathcal{W}^{\prime \prime}\left(\boldsymbol{A}^{(x)}\right)\right) \mathrm{d} \mu(x)=\mathcal{A}$.

We leave this as a simple exercise that the whole assertion of the proposition now easily follows.

As an important for us consequence of Proposition 20.6 we now obtain

20.7. Corollary. Let $(\mu, \Phi) \in \operatorname{RGS}(X, \mathfrak{M}),(\nu, \Psi) \in \operatorname{RGS}(Y, \mathfrak{N})$ and let $\widehat{\mu}=\Phi^{*}(\mu)$ and $\widehat{\nu}=\Psi^{*}(\nu)$. For

$$
\mathbb{X}=\int_{X}^{\boxplus} \Phi(x) \mathrm{d} \mu(x) \quad \text { and } \quad \mathbb{Y}=\int_{Y}^{\boxplus} \Psi(y) \mathrm{d} \nu(y)
$$

we have:

(a) $\mathbb{X}=\mathbb{Y} \Longleftrightarrow \widehat{\mu} \ll \widehat{\nu} \ll \widehat{\mu}$,

(b) $\mathbb{X} \leqslant^{s} \mathbb{Y} \Longleftrightarrow \widehat{\mu} \ll \widehat{\nu}$. 
Proof. We know that $\mathbb{X}=\int_{\mathfrak{F}_{N}}^{\boxplus} \mathbb{F} \mathrm{d} \widehat{\mu}(\mathbb{F})$ and $\mathbb{Y}=\int_{\mathfrak{F}_{N}}^{\boxplus} \mathbb{F} \mathrm{d} \widehat{\nu}(\mathbb{F})$. Observe that point (b) follows from (a) and Proposition 20.6, and implication ' $\Longleftarrow$ ' in (a) is a consequence of (DI4). To prove the converse one, assume $\boldsymbol{X}=\int_{X}^{\boxplus} \boldsymbol{A}^{(x)} \mathrm{d} \mu(x)$ with $\mathbb{A}^{(x)}=\Phi(x)$ for $\mu$-almost all $x \in X$, $\boldsymbol{Y}=\int_{Y}^{\boxplus} \boldsymbol{B}^{(y)} \mathrm{d} \nu(y)$ with $\mathbb{B}^{(y)}=\Psi(y)$ for $\nu$-almost all $y \in Y$, and $U$ is a unitary operator such that $U \cdot X \cdot U^{-1}=Y$. It then follows from Proposition 20.6 that $U$ sends the algebra of all diagonalizable operators on $\overline{\mathcal{D}}(\boldsymbol{X})$ onto the algebra of all diagonalizable operators on $\bar{D}(\boldsymbol{Y})$. Thus, according to [34, Theorem IV.8.23], there is a Borel isomorphism $\kappa: Y \backslash Y_{0} \rightarrow X \backslash X_{0}$ where $X_{0} \in \mathcal{N}(\mu)$ and $Y_{0} \in \mathcal{N}(\nu)$ such that

$$
\kappa^{*}(\nu) \ll \mu \ll \kappa^{*}(\nu)
$$

and $U$ may be written in the form $U=\int_{X}^{\oplus} U_{x} \sqrt{\frac{\mathrm{d} \kappa^{*}(\nu)}{\mathrm{d} \mu}(x)} \mathrm{d} \mu(x)$ where $\left\{U_{x}\right\}_{x \in X}$ is a certain measurable field of unitary operators (for the details we refer to Takesaki's book [34]). Since $U \cdot \mathfrak{b}(\boldsymbol{X})=\mathfrak{b}(Y) \cdot U$, we conclude from (di1) (page 76) that $\int_{X}^{\oplus} U_{x} \cdot \mathfrak{b}\left(A^{(x)}\right) \sqrt{\frac{\mathrm{d} \kappa^{*}(\nu)}{\mathrm{d} \mu}(x)} \mathrm{d} \mu(x)=$ $\int_{X}^{\oplus} \mathfrak{b}\left(\boldsymbol{B}^{(\kappa(x))}\right) \cdot U_{x} \sqrt{\frac{\mathrm{d} \kappa^{*}(\nu)}{\mathrm{d} \mu}(x)} \mathrm{d} \mu(x)$. Now thanks to the uniqueness of the decomposition of a bounded decomposable operator and positivity of the function $\sqrt{\frac{\mathrm{d} \kappa^{*}(\nu)}{\mathrm{d} \mu}}$, the latter equation implies that $U_{x} \cdot \mathfrak{b}\left(A^{(x)}\right)=$ $\mathfrak{b}\left(\boldsymbol{B}^{(\kappa(x))}\right) \cdot U_{x}$ for $\mu$-almost all $x \in X$. Consequently, $\mathbb{B}^{(\kappa(x))}=\mathbb{A}^{(x)}$ for $\mu$-almost all $x \in X$. We leave this as an exercise that the latter combined with (20-7) gives $\widehat{\mu} \ll \widehat{\nu} \ll \widehat{\mu}$ which finishes the proof.

A similar result was obtained by Ernest (cf. [9, Theorem 3.8]). However, he was working (when speaking of the central decomposition of an operator) with quasi-equivalence classes instead of unitary equivalence ones.

To avoid repetitions, let us say a function $f: X \rightarrow I_{\aleph_{0}}$ fits to $(\mu, \Phi) \in$ $\operatorname{RGS}(X, \mathfrak{M})$ iff $f$ is almost measurable and there are disjoint measurable sets $X_{1}$ and $X_{2}$ such that $\mu\left(X \backslash\left(X_{1} \cup X_{2}\right)\right)=0, f\left(X_{1}\right) \subset$ Card and $\Phi\left(X_{2}\right) \subset \mathfrak{s}_{N}$. Note that if the latter happens, the function $f \odot \Phi$ given by $(f \odot \Phi)(x)=f(x) \odot \Phi(x)$ is well defined on $X_{1} \cup X_{2}$.

20.8. Lemma. Let $(\mu, \Phi) \in \operatorname{RGS}(X, \mathfrak{M})$ and $f: X \rightarrow I_{\aleph_{0}} \backslash\{0\}$ be a function which fits to $(\mu, \Phi)$. Then $(\mu, f \odot \Phi) \in \operatorname{RGS}(X, \mathfrak{M})$ as well.

Proof. It follows from Theorem 19.8 (page 74) that $f \odot \Phi$ is integrable. Further, we infer from (DI3) (page 77) that $\aleph_{0} \odot \int_{D}^{\oplus} f(x) \odot \Phi(x) \mathrm{d} \mu(x)=$ $\int_{D}^{\oplus}\left(\aleph_{0} \cdot f(x)\right) \odot \Phi(x) \mathrm{d} \mu(x)=\aleph_{0} \odot \int_{D}^{\oplus} \Phi(x) \mathrm{d} \mu(x)$ and thus $\int_{D}^{\oplus} f(x) \odot$ $\Phi(x) \mathrm{d} \mu(x) \perp_{u} \int_{X \backslash D}^{\oplus} f(x) \odot \Phi(x) \mathrm{d} \mu(x)$ since

$$
\int_{D}^{\oplus} \Phi(x) \mathrm{d} \mu(x) \perp_{u} \int_{X \backslash D}^{\oplus} \Phi(x) \mathrm{d} \mu(x) .
$$


Whenever a function $f: X \rightarrow I_{\aleph_{0}}$ fits to $(\mu, \Phi) \in \operatorname{RGS}(X, \mathfrak{M})$, we define $\int_{X}^{\boxplus} f(x) \odot \Phi(x) \mathrm{d} \mu(x)$ as follows. Put $s(f)=\{x \in X: f(x)>0\}$ and take $X_{0} \in \mathcal{N}(\mu)$ such that $f$ is measurable on $X \backslash X_{0}$. If $\mu(s(f) \backslash$ $\left.X_{0}\right)>0, \int_{X}^{\boxplus} f(x) \odot \Phi(x) \mathrm{d} \mu(x)$ denotes $\int_{s(f) \backslash X_{0}}^{\boxplus} f(x) \odot \Phi(x) \mathrm{d} \mu(x)$ (see Lemma 20.8). Otherwise let $\int_{X}^{\boxplus} f(x) \odot \Phi(x) \mathrm{d} \mu(x)=\mathbb{O}$. The usage of ' $\int^{\boxplus}$ here is justified by Lemma 20.8 .

Below we formulate a variation of [9, Proposition 3.2]. We shall use it in our theorem on prime decomposition.

20.9. Lemma. Let $\mathbb{A} \in \mathcal{S} \mathcal{E} \mathcal{P}_{N}$ be the direct sum of a minimal $N$-tuple and a semiminimal one.

(A) There is $\mu_{\mathbb{A}} \in \operatorname{rgm}\left(\mathfrak{p}_{N}\right)$ such that $\mathbb{A}=\int_{\mathfrak{p}_{N}}^{\boxplus} \mathbb{P} \mathrm{d} \mu_{\mathbb{A}}(\mathbb{P})$. For $\mu \in$ $\operatorname{rgm}\left(\mathfrak{p}_{N}\right), \mathbb{A}=\int_{\mathfrak{p}_{N}}^{\boxplus} \mathbb{P} \mu(\mathbb{P}) \Longleftrightarrow \mu \ll \mu_{\mathbb{A}} \ll \mu$.

(B) For $\mathbb{B} \in \mathcal{S} \mathcal{E} \mathcal{P}_{N}$ the following conditions are equivalent:

(i) $\mathbb{B} \ll \mathbb{A}$,

(ii) there is an almost measurable function $f: \mathfrak{p}_{N} \rightarrow I_{\aleph_{0}}$ such that $f\left(\mathfrak{a}_{N}\right) \subset$ Card, $f\left(\mathfrak{f}_{N}\right) \subset\left\{0, \aleph_{0}\right\}$ and

$$
\mathbb{B}=\int_{\mathfrak{p}_{N}}^{\boxplus} f(\mathbb{P}) \odot \mathbb{P} \mathrm{d} \mu_{\mathbb{A}}(\mathbb{P}) .
$$

(C) Let $(\mu, \Phi) \in \operatorname{RGS}(X, \mathfrak{M})$.

(a) If $\Phi(X) \subset \mathfrak{a}_{N}, \int_{X}^{\boxplus} \Phi(x) \mathrm{d} \mu(x) \in \mathcal{M} \mathcal{F}_{N}$.

(b) If $\Phi(X) \subset \mathfrak{f}_{N}, \int_{X}^{\boxplus} \Phi(x) \mathrm{d} \mu(x) \in \mathcal{H}^{\boxplus} \mathcal{J M}_{N}$.

(c) If $\Phi(X) \subset \mathfrak{s}_{N}$ and $f: X \rightarrow \mathbb{R}_{+}$is almost measurable, $\int_{X}^{\boxplus} f(x) \odot$ $\Phi(x) \mathrm{d} \mu(x) \in \mathcal{S \mathcal { M }}_{N}$.

Proof. Let $\mathbb{F}$ be an arbitrary member of $\mathcal{S} \mathcal{E} \mathcal{P}_{N}$ and let $\boldsymbol{F}$ be a representative of $\mathbb{F}$. It follows from the reduction theory of von Neumann algebras (see e.g. [34, Theorem IV.8.21]) that there is a standard Borel space $(X, \mathfrak{M})$ with a probabilistic Borel measure $\lambda$ and a measurable field $\left\{\mathcal{M}_{x}\right\}_{x \in X}$ of factors (each of which acts on some $\mathcal{H}_{n}$ ) such that the von Neumann algebras $\mathcal{M}:=\int_{X}^{\oplus} \mathcal{M}_{x} \mathrm{~d} \lambda(x)$ and $\mathcal{W}^{\prime \prime}(\boldsymbol{F})$ are spatially isomorphic. Write $\mathfrak{b}(\boldsymbol{F})=\left(T_{1}, \ldots, T_{N}\right) . T_{j}$ corresponds (under the spatial isomorphism) to $T_{j}^{\prime} \in \mathcal{M}$. Since then $\mathfrak{b}(\boldsymbol{F}) \equiv\left(T_{1}^{\prime}, \ldots, T_{N}^{\prime}\right)$, we see that there is $\boldsymbol{F}^{\prime} \in \mathrm{CDD}_{N}$ such that $\mathfrak{b}\left(\boldsymbol{F}^{\prime}\right)=\left(T_{1}^{\prime}, \ldots, T_{N}^{\prime}\right)$ and consequently $\boldsymbol{F}^{\prime} \equiv \boldsymbol{F}$. Thus replacing $\boldsymbol{F}$ by $\boldsymbol{F}^{\prime}$, we may assume that $\mathcal{W}^{\prime \prime}(\boldsymbol{F})=\mathcal{M}$. Write $T_{j}=\int_{X}^{\oplus} T_{j}^{(x)} \mathrm{d} \lambda(x)$ where $T_{j}^{(x)} \in \mathcal{M}_{x}$ for $\lambda$-almost all $x \in X$. Since $\left\|T_{j}\right\| \leqslant 1$, we also have $\left\|T_{j}^{(x)}\right\| \leqslant 1 \lambda$-almost everywhere. Further, the function $x \mapsto \mathcal{N}\left(I-\left(T_{j}^{(x)}\right)^{*} T_{j}^{(x)}\right)$ is measurable (in the target space we consider the Effros Borel structure separately on each of $\mathcal{H}_{n}$ 's) and hence the set $X_{0}=\left\{x \in X: \mathcal{N}\left(I-\left(T_{j}^{(x)}\right)^{*} T_{j}^{(x)}\right) \neq\{0\}\right\}$ is measurable. Suppose $\lambda\left(X_{0}\right)>0$. Then there exists a measurable vector field 
$x \mapsto \xi_{x}$ such that $\xi_{x} \in \mathcal{N}\left(I-\left(T_{j}^{(x)}\right)^{*} T_{j}^{(x)}\right)$ and $\left\|\xi_{x}\right\| \leqslant 1$ for $\lambda$-almost all $x \in X$, and $\int_{X}\left\|\xi_{x}\right\|^{2} \mathrm{~d} \lambda(x)>0$ (see Corollary after Theorem 2 in [6]; or [34, Corollary IV.8.3]). We infer from this that $\xi=\int_{X}^{\oplus} \xi_{x} \mathrm{~d} \lambda(x)$ is well defined and nonzero, and $T_{j}^{*} T_{j} \xi=\xi$ which denies the fact that $T_{j}$ is a value of the $\mathfrak{b}$-transform. This shows that $\lambda\left(X_{0}\right)=0$ and hence for $\lambda$-almost all $x \in X$ there is an operator $F_{j}^{(x)} \in$ CDD such that $\mathfrak{b}\left(F_{j}^{(x)}\right)=T_{j}^{(x)}$. Put $\boldsymbol{F}^{(x)}=\left(F_{1}^{(x)}, \ldots, F_{N}^{(x)}\right)$ and observe that the function $x \mapsto \boldsymbol{F}^{(x)}$ is measurable (since the $\mathfrak{b}$-transform is an isomorphism) and $\boldsymbol{F}=\int_{X}^{\oplus} \boldsymbol{F}^{(x)} \mathrm{d} \lambda(x)$. Since the field $x \mapsto \mathcal{W}^{\prime \prime}\left(\boldsymbol{F}^{(x)}\right)$ is measurable and $\mathcal{W}^{\prime \prime}\left(\boldsymbol{F}^{(x)}\right) \subset \mathcal{M}_{x}, \int_{X}^{\oplus} \mathcal{W}^{\prime \prime}\left(\boldsymbol{F}^{(x)}\right) \mathrm{d} \lambda(x) \subset \mathcal{M}=\mathcal{W}^{\prime \prime}(\boldsymbol{F})$. At the same time, $T_{1}, \ldots, T_{N} \in \int_{X}^{\oplus} \mathcal{W}^{\prime \prime}\left(\boldsymbol{F}^{(x)}\right) \mathrm{d} \lambda(x)$ and therefore $\mathcal{W}^{\prime \prime}(\boldsymbol{F}) \subset$ $\int_{X}^{\oplus} \mathcal{W}^{\prime \prime}\left(\boldsymbol{F}^{(x)}\right) \mathrm{d} \lambda(x)$ as well. We conclude from this that $\mathcal{W}^{\prime \prime}\left(\boldsymbol{F}^{(x)}\right)=\mathcal{M}_{x}$ for $\lambda$-almost all $x \in X$ and consequently $\int_{X}^{\oplus} \mathcal{W}^{\prime \prime}\left(\boldsymbol{F}^{(x)}\right) \mathrm{d} \lambda(x)$ is the central decomposition of $\mathcal{W}^{\prime \prime}(\boldsymbol{F})$. In particular, $\mathbb{F}^{(x)} \in \mathfrak{F}_{N}$ for $\lambda$-almost all $x \in X$. Now Proposition 20.6 implies that $\mathbb{F}=\int_{X}^{\boxplus} \Phi(x) \mathrm{d} \lambda(x)$ where $\Phi: X \ni x \mapsto \mathbb{F}^{(x)} \in \mathfrak{F}_{N}$. Let $\mu_{\mathbb{F}}=\Phi^{*}(\lambda) \in \operatorname{rgm}\left(\mathfrak{F}_{N}\right)$. We know that then

$$
\mathbb{F}=\int_{\mathfrak{F}_{N}}^{\boxplus} \mathbb{X} \mathrm{d} \mu_{\mathbb{F}}(\mathbb{X}) .
$$

Further, since central decompositions of von Neumann algebras preserve the types ([18, Theorem 14.1.21] or [34, Corollary V.6.7]), we infer from this that $\mathbb{F}$ is type I, $\mathrm{I}^{n}, \mathrm{II}, \mathrm{II}^{1}, \mathrm{II}^{\infty}$ or III iff $\mu_{\mathbb{F}^{-}}$-almost all $\mathbb{X} \in \mathfrak{F}_{N}$ are such. In particular, if $\mathbb{F}$ is the direct sum of a minimal $N$ tuple and a semiminimal one, $\mathcal{W}^{\prime \prime}(\boldsymbol{F})$ decomposes into type $\mathrm{I}_{1}, \mathrm{II}_{1}$ and III parts (and no other parts) and consequently $\mu_{\mathbb{F}}$-almost all $\mathbb{X} \in \mathfrak{F}_{N}$ are type $\mathrm{I}^{1}$ (atoms) or $\mathrm{II}^{1}$ (semiprimes), or III (fractals) - cf. Propositions 17.3 (page 60) and 17.6 (page 61). This proves the first claim of (A). The remainder of (A) follows from Corollary 20.7.

We pass to (B). First of all, note that (20-8) makes sense thanks to Lemma 20.8. Suppose $\mathbb{B}$ is given by (20-8). We may assume that $f$ is measurable. Then $s(f)=\left\{\mathbb{P} \in \mathfrak{p}_{N}: f(\mathbb{P})>0\right\} \in \mathfrak{B}_{N}$. It follows from (DI3) (page 77) that $\aleph_{0} \odot \mathbb{B}=\int_{\mathfrak{p}_{N}}^{\oplus}\left(\aleph_{0} \cdot f(\mathbb{P})\right) \odot \mathbb{P} \mathrm{d} \mu_{\mathbb{A}}(\mathbb{P})=$ $\aleph_{0} \odot \int_{s(f)}^{\oplus} \mathbb{P} \mathrm{d} \mu_{\mathbb{A}}(\mathbb{P}) \leqslant \aleph_{0} \odot \mathbb{A}$ and thus $\mathbb{B} \ll \mathbb{A}$.

Now assume that $\mathbb{B} \ll \mathbb{A}$. Let $\mu_{\mathbb{B}} \in \operatorname{rgm}\left(\mathfrak{F}_{N}\right)$ be as in (20-9) with $\mathbb{F}=$ $\mathbb{B}$. Since $\mathbb{B} \ll \mathbb{A}$ and $\mathbb{A}, \mathbb{B} \in \mathcal{S} \mathcal{E} \mathcal{P}_{N}, \aleph_{0} \odot \mathbb{B} \leqslant^{s} \aleph_{0} \odot \mathbb{A}$ (cf. Corollary 11.5, page 27). So, (PR6) (page 9) and Proposition 20.6 yield that there is a measurable set $\mathcal{B} \subset \mathfrak{p}_{N}$ such that $\aleph_{0} \odot \mathbb{B}=\aleph_{0} \odot \int_{\mathcal{B}}^{\boxplus} \mathbb{P} \mathrm{d} \mu_{\mathbb{A}}(\mathbb{P})$. Now we infer from (DI3) and Lemma 20.8 that

$$
\int_{\mathfrak{F}_{N}}^{\boxplus} \aleph_{0} \odot \mathbb{F} \mathrm{d} \mu_{\mathbb{B}}(\mathbb{F})=\int_{\mathcal{B}}^{\boxplus} \aleph_{0} \odot \mathbb{P} \mathrm{d} \mu_{\mathbb{A}}(\mathbb{P}) .
$$


An application of Proposition 20.4 shows that there are measurable domains $\mathcal{F}_{0} \subset \mathcal{B}$ and $\mathcal{G}_{0} \subset \mathfrak{F}_{N}$ such that $\mu_{\mathbb{A}}\left(\mathcal{B} \backslash \mathcal{F}_{0}\right)=0, \mu_{\mathbb{B}}\left(\mathfrak{F}_{N} \backslash \mathcal{G}_{0}\right)=$ $0, \mathcal{F}_{0}^{*}=\left\{\aleph_{0} \odot \mathbb{P}: \mathbb{P} \in \mathcal{F}_{0}\right\} \in \mathfrak{B}_{N}, \mathcal{G}_{0}^{*}=\left\{\aleph_{0} \odot \mathbb{F}: \mathbb{F} \in \mathcal{G}_{0}\right\} \in \mathfrak{B}_{N}$, the sets $\mathcal{F}_{0}, \mathcal{G}_{0}, \mathcal{F}_{0}^{*}$ and $\mathcal{G}_{0}^{*}$ are standard Borel spaces and the functions $\Phi: \mathcal{F}_{0} \ni$ $\mathbb{P} \mapsto \aleph_{0} \odot \mathbb{P} \in \mathcal{F}_{0}^{*}$ and $\Psi: \mathcal{G}_{0} \ni \mathbb{F} \mapsto \aleph_{0} \odot \mathbb{F} \in \mathcal{G}_{0}^{*}$ are Borel isomorphisms. Put $\mathcal{F}=\Phi^{-1}\left(\mathcal{F}_{0}^{*} \cap \mathcal{G}_{0}^{*}\right) \in \mathfrak{B}_{N}$ and $\mathcal{G}=\Psi^{-1}\left(\mathcal{F}_{0}^{*} \cap \mathcal{G}_{0}^{*}\right) \in \mathfrak{B}_{N}$. Let $\Theta=\left.\Psi^{-1} \circ \Phi\right|_{\mathcal{F}}$. Observe that $\Theta$ is a Borel isomorphism of $\mathcal{F}$ onto $\mathcal{G}$. One may deduce from Corollary 20.7 and (20-10) that $\mu_{\mathbb{A}}(\mathcal{B} \backslash \mathcal{F})=0$ and $\mu_{\mathbb{B}}\left(\mathfrak{F}_{N} \backslash \mathcal{G}\right)=0$, and $\left.\lambda \ll \mu_{\mathbb{A}}\right|_{\mathcal{F}} \ll \lambda$ where $\lambda(\sigma)=\mu_{\mathbb{B}}(\Theta(\sigma \cap \mathcal{F}))$ for measurable $\sigma \subset \mathfrak{p}_{N}$. Consequently (by (DI4), page 77),

$$
\mathbb{Y}=\int_{\mathcal{F}}^{\boxplus} \Theta(\mathbb{P}) \mathrm{d} \mu_{\mathbb{A}}(\mathbb{P}) .
$$

Since $\Theta(\mathbb{P}) \ll \mathbb{P}$ for any $\mathbb{P} \in \mathcal{F}$, we may define $f: \mathfrak{p}_{N} \rightarrow I_{\aleph_{0}}$ by $f(\mathbb{P})=$ $\Theta(\mathbb{P}): \mathbb{P}$ for $\mathbb{P} \in \mathcal{F}$ and $f(\mathbb{P})=0$ for $\mathbb{P} \in \mathfrak{p}_{N} \backslash \mathcal{F}$. Thanks to $(20-11)$, it suffices to show that $\left.f\right|_{\mathcal{F}}$ is measurable. Since $\mathcal{F}$ and $\mathcal{G}$ are standard Borel spaces, the graph $\Gamma=\{(\mathbb{P}, \Theta(\mathbb{P})): \mathbb{P} \in \mathcal{F}\}$ of $\Theta$ is a Borel subset of $\mathcal{F} \times \mathcal{G}$ and $u: \mathcal{F} \ni \mathbb{P} \mapsto(\mathbb{P}, \Theta(\mathbb{P})) \in \Gamma$ is a Borel isomorphism. Finally, since Div is Borel (see Section 19, page 74), so is the function $v: \Gamma \ni(\mathbb{A}, \mathbb{B}) \mapsto \mathbb{B}: \mathbb{A} \in I_{\aleph_{0}}$ (here is important that $\mathcal{F}$ and $\mathcal{G}$ are standard Borel spaces). The notice that $\left.f\right|_{\mathcal{F}}=v \circ u$ finishes the proof.

Finally, point (C) follows from Proposition 20.6 and the previously mentioned fact that central decompositions of von Neumann algebras preserve the types.

The formula (20-9) corresponds to Ernest's central decomposition of a bounded operator [9, Chapter III]. It is however not of our interest. Also a variation of (20-8) appears in [9, Lemma 4.4].

In the sequel we shall need one more result.

20.10. Lemma. For $\mu, \nu \in \operatorname{rgm}\left(\mathfrak{F}_{N}\right)$ the following conditions are equivalent:

(i) $\int_{\mathfrak{F}_{N}}^{\boxplus} \mathbb{F} \mathrm{d} \mu(\mathbb{F}) \perp_{u} \int_{\mathfrak{F}_{N}}^{\boxplus} \mathbb{F} \mathrm{d} \nu(\mathbb{F})$,

(ii) there are measurable sets $\mathcal{A}, \mathcal{B} \subset \mathfrak{F}_{N}$ such that $\mu\left(\mathfrak{F}_{N} \backslash \mathcal{A}\right)=0$, $\nu\left(\mathfrak{F}_{N} \backslash \mathcal{B}\right)=0$ and $\mathcal{A} \perp_{u} \mathcal{B}$,

(iii) there are measurable sets $\mathcal{A}, \mathcal{B} \subset \mathfrak{F}_{N}$ such that $\mu\left(\mathfrak{F}_{N} \backslash \mathcal{A}\right)=0$, $\nu\left(\mathfrak{F}_{N} \backslash \mathcal{B}\right)=0$ and $\mathcal{A} \perp_{s} \mathcal{B}$,

(iv) $\mu \perp \nu$ and $\mu+\nu \in \operatorname{rgm}\left(\mathfrak{F}_{N}\right)$.

Proof. (i) $\Longrightarrow$ (iv): Put $\mathbb{A}=\int_{\mathfrak{F}_{N}}^{\boxplus} \mathbb{X} \mathrm{d} \mu(\mathbb{X}), \mathbb{B}=\int_{\mathfrak{F}_{N}}^{\boxplus} \mathbb{X} \nu(\mathbb{X})$ and $\mathbb{F}=$ $\mathbb{A} \boxplus \mathbb{B}$, and let $\lambda=\mu_{\mathbb{F}}$ where $\mu_{\mathbb{F}}$ is as in (20-9). Since $\mathbb{A}, \mathbb{B} \leqslant^{s} \mathbb{F}$, we infer from Corollary 20.7 that $\mu, \nu \ll \lambda$. So, $\mu+\nu \ll \lambda$ and therefore $\mu+\nu \in \operatorname{rgm}\left(\mathfrak{F}_{N}\right)$. Further, there are measurable sets $\mathcal{A}, \mathcal{B} \subset \mathfrak{F}_{N}$ such that $\left.\mu \ll \lambda\right|_{\mathcal{A}} \ll \mu$ and $\left.\nu \ll \lambda\right|_{\mathcal{B}} \ll \nu$ and consequently, again by Corollary 20.7, $\mathbb{A}=\int_{\mathcal{A}}^{\boxplus} \mathbb{X} \mathrm{d} \lambda(\mathbb{X})$ and $\mathbb{B}=\int_{\mathcal{B}}^{\boxplus} \mathbb{X} \mathrm{d} \lambda(\mathbb{X})$. Since then $\int_{\mathcal{A} \cap \mathcal{B}}^{\boxplus} \mathbb{X} \mathrm{d} \lambda(\mathbb{X}) \leqslant^{s} \mathbb{A}, \mathbb{B}$, one has $\lambda(\mathcal{A} \cap \mathcal{B})=0$ and hence $\mu \perp \nu$. 
(iv) $\Longrightarrow$ (iii): Put $\lambda=\mu+\nu$ and let $\mathcal{A}_{0}$ and $\mathcal{B}_{0}$ be disjoint measurable subsets of $\mathfrak{F}_{N}$ on which (respectively) $\mu$ and $\nu$ are concentrated. Since $\lambda \in \operatorname{rgm}\left(\mathfrak{F}_{N}\right), \int_{\mathcal{A}_{0}}^{\oplus} \mathbb{F} d \lambda(\mathbb{F}) \perp_{u} \int_{\mathcal{B}_{0}}^{\oplus} \mathbb{F} \mathrm{d} \lambda(\mathbb{F})$ which yields (cf. Proposition 18.3, page 65, and the proof of Proposition 20.4, page 78, or [29, Proposition 3.2.7]) that there exist a sequence $\left(p_{n}\right)_{n=1}^{\infty} \subset \mathcal{P}_{1}(N)$ and a set $\mathcal{Z} \in \mathcal{N}(\lambda)$ such that $p_{n}\left(\mathfrak{b}(\mathbb{F}), \mathfrak{b}(\mathbb{F})^{*}\right) \stackrel{* s}{\rightarrow} j(\mathbb{F}) I$ for each $\mathbb{F} \in$ $\left(\mathcal{A}_{0} \cup \mathcal{B}_{0}\right) \backslash \mathcal{Z}$ where $j$ is the characteristic function of $\mathcal{A}_{0}$. Consequently, $\mu$ and $\nu$ are concentrated on, respectively, $\mathcal{A}=\mathcal{A}_{0} \backslash \mathcal{Z}$ and $\mathcal{B}=\mathcal{B}_{0} \backslash \mathcal{Z}$, and $\mathcal{A} \perp_{s} \mathcal{B}$.

Since (ii) obviously follows from (iii), it remains to show that (i) is implied by (ii). Suppose (i) is false. This means that there are nontrivial $N$-tuples $\mathbb{A} \leqslant s \int_{\mathfrak{F}_{N}}^{\boxplus} \mathbb{F} \mu(\mathbb{F})$ and $\mathbb{B} \leqslant^{s} \int_{\mathfrak{F}_{N}}^{\boxplus} \mathbb{F} \mathrm{d} \nu(\mathbb{F})$ such that $\aleph_{0} \odot \mathbb{A}=\aleph_{0} \odot \mathbb{B}$. By Corollary 20.7, there are measurable sets $\mathcal{A}_{1}, \mathcal{B}_{1} \subset$ $\mathfrak{F}_{N}$ such that $\mathbb{A}=\int_{\mathcal{A}_{1}}^{\boxplus} \mathbb{F} \mathrm{d} \mu(\mathbb{F})$ and $\mathbb{B}=\int_{\mathcal{B}_{1}}^{\boxplus} \mathbb{\mathrm { F }} \nu(\mathbb{F})$. All these combined with (DI3) (page 77) and Lemma 20.8 give

$$
\int_{\mathcal{A}_{1} \cap \mathcal{A}}^{\boxplus} \aleph_{0} \odot \mathbb{F} \mathrm{d} \mu(\mathbb{F})=\int_{\mathcal{B}_{1} \cap \mathcal{B}}^{\boxplus} \aleph_{0} \odot \mathbb{F} \mathrm{d} \nu(\mathbb{F})
$$

where $\mathcal{A}$ and $\mathcal{B}$ are as in (ii). Thanks to Proposition 20.4 (page 78), we may assume that $\mathcal{F}=\left\{\aleph_{0} \odot \mathbb{F}: \mathbb{F} \in \mathcal{A}_{1} \cap \mathcal{A}\right\}$ and $\mathcal{G}=\left\{\aleph_{0} \odot \mathbb{F}: \mathbb{F} \in\right.$ $\left.\mathcal{B}_{1} \cap \mathcal{B}\right\}$ are measurable. We conclude from unitary disjointness of $\mathcal{A}$ and $\mathcal{B}$ that

$$
\Phi^{*}(\mu)(\mathcal{G})=0 \quad \text { and } \quad \Phi^{*}(\nu)(\mathcal{F})=0
$$

where $\Phi: \mathfrak{F}_{N} \ni \mathbb{F} \mapsto \aleph_{0} \odot \mathbb{F} \in \mathfrak{F}_{N}$. But (20-12) yields, by Corollary 20.7, that $\Phi^{*}(\mu) \ll \Phi^{*}(\nu) \ll \Phi^{*}(\mu)$. Consequently, it follows from (20-13) that $\mu\left(\mathcal{A}_{1} \cap \mathcal{A}\right)=0$ and $\nu\left(\mathcal{B} \cap \mathcal{B}_{1}\right)=0$ which denies the fact that $\mathbb{A}$ and $\mathbb{B}$ were nonzero.

Taking into account the above result, for arbitrary two measures $\mu, \nu \in \operatorname{rgm}\left(\mathfrak{F}_{N}\right)$ we shall write $\mu \perp_{s} \nu$ iff any of the equivalent conditions (i)-(iv) of Lemma 20.10 is fulfilled.

\section{1. 'Continuous' Direct Sums}

Property (DI4) (page 77) suggests replacing standard measures $\mu$ by their null $\sigma$-ideals $\mathcal{N}(\mu)$. In this section we follow this concept. In that way we shall extend the notion of the (standard 'discrete') direct sum to more general context. We begin with

21.1. Definition. A measurable space with nullity is a triple $(\mathscr{X}, \mathfrak{M}, \mathcal{N})$ where $(\mathscr{X}, \mathfrak{M})$ is a measurable space and $\mathcal{N}$ is a $\sigma$-ideal in $\mathfrak{M}$; that is, $\varnothing \in \mathcal{N} \subset \mathfrak{M}, \bigcup_{n=1}^{\infty} A_{n} \in \mathcal{N}$ whenever $\left\{A_{n}\right\}_{n=1}^{\infty} \subset \mathcal{N}$ and $\{B \in \mathfrak{M}: B \subset$ $A\} \subset \mathcal{N}$ for every $A \in \mathcal{N}$.

Whenever $(\mathscr{X}, \mathfrak{M}, \mathcal{N})$ is a measurable space with nullity, $\overline{\mathcal{N}}$ denotes the family of all (possibly nonmeasurable) sets which are contained in members of $\mathcal{N}$. Members of $\overline{\mathcal{N}}$ are called null sets, the other subsets 
of $\mathscr{X}$ are called nonnull. For $Y \in \mathfrak{M},\left(Y,\left.\mathfrak{M}\right|_{Y},\left.\mathcal{N}\right|_{Y}\right)$ is the induced measurable space with nullity, i.e. $\left.\mathfrak{M}\right|_{Y}=\{B \in \mathfrak{M}: B \subset Y\}$ and $\left.\mathcal{N}\right|_{Y}=\left.\mathfrak{M}\right|_{Y} \cap \mathcal{N}$. The space $(\mathscr{X}, \mathfrak{M}, \mathcal{N})$ is trivial iff $\mathscr{X} \in \mathcal{N}$.

A function $\Phi: \mathscr{X}_{1} \rightarrow \mathscr{X}_{2}$ is a null-isomorphism between measurable spaces with nullities $\left(\mathscr{X}_{1}, \mathfrak{M}_{1}, \mathcal{N}_{1}\right)$ and $\left(\mathscr{X}_{2}, \mathfrak{M}_{2}, \mathcal{N}_{2}\right)$ if $\Phi$ is a Borel isomorphism such that $\mathcal{N}_{2}=\left\{\Phi(Z): Z \in \mathcal{N}_{1}\right\}$. If $\Psi: X_{1} \rightarrow \mathscr{X}_{2}$ (with $X_{1} \subset \mathscr{X}_{1}$ ) is a function such that there are sets $Z_{1} \in \mathcal{N}_{1}$ and $Z_{2} \in \mathcal{N}_{2}$ for which $\mathscr{X}_{1} \backslash Z_{1} \subset X_{1}$ and $\left.\Psi\right|_{\mathscr{X}_{1} \backslash Z_{1}}$ is a null-isomorphism of $\left(\mathscr{X}_{1} \backslash Z_{1},\left.\mathfrak{M}_{1}\right|_{\mathscr{X}_{1} \backslash Z_{1}},\left.\mathcal{N}_{1}\right|_{\mathscr{X}_{1} \backslash Z_{1}}\right)$ onto $\left(\mathscr{X}_{2} \backslash Z_{2},\left.\mathfrak{M}_{2}\right|_{\mathscr{X}_{2} \backslash Z_{2}},\left.\mathcal{N}_{2}\right|_{\mathscr{X}_{2} \backslash Z_{2}}\right), \Psi$ is said to be an almost null-isomorphism and spaces $\left(\mathscr{X}_{1}, \mathfrak{M}_{1}, \mathcal{N}_{1}\right)$ and $\left(\mathscr{X}_{2}, \mathfrak{M}_{2}, \mathcal{N}_{2}\right)$ are almost isomorphic. Similarly, a function $u: X \rightarrow Y$ (where $X \subset \mathscr{X},(\mathscr{X}, \mathfrak{M}, \mathcal{N})$ is a measurable space with nullity and $(Y, \mathfrak{N})$ is a measurable space) is said to be almost measurable iff there is a set $X^{\prime} \in \mathfrak{M}$ contained in $X$ such that $X \backslash X^{\prime} \in \overline{\mathcal{N}}$ and $\left.u\right|_{X^{\prime}}$ is measurable.

Our main interest are measurable spaces whose nullities come from certain measures. For this purpose we introduce

21.2. Definition. Let $(\mathscr{X}, \mathfrak{M}, \mathcal{N})$ be a measurable space with nullity. A measurable set $A \subset \mathscr{X}$ is standard iff $\left(A,\left.\mathfrak{M}\right|_{A},\left.\mathcal{N}\right|_{A}\right)$ is almost isomorphic to $(Y, \mathfrak{N}, \mathcal{N}(\nu))$ for some standard measure space $(Y, \mathfrak{N}, \nu)$. Standard sets are nonnull.

A family $\mathcal{B}$ is said to be a base of $(\mathscr{X}, \mathfrak{M}, \mathcal{N})$ iff the following two conditions are fulfilled:

- $\mathcal{B}$ consists of pairwise disjoint measurable sets and $\mathscr{X} \backslash \bigcup \mathcal{B} \in$ $\mathcal{N}$,

- for any $A \subset \bigcup \mathcal{B}, A \in \mathfrak{M}$ (respectively $A \in \mathcal{N}$ ) iff $A \cap B \in \mathfrak{M}$ $(A \cap B \in \mathcal{N})$ for any $B \in \mathcal{B}$.

A base is standard iff it consists of standard sets. $(\mathscr{X}, \mathfrak{M}, \mathcal{N})$ is called multi-standard iff it admits a standard base.

Let $\mathscr{F}=\left\{\left(\mathscr{X}_{s}, \mathfrak{M}_{s}, \mathcal{N}_{s}\right)\right\}_{s \in S}$ be a family of measurable spaces with nullities. The direct sum of $\mathscr{F}$, denoted by $\bigoplus_{s \in S}\left(\mathscr{X}_{s}, \mathfrak{M}_{s}, \mathcal{N}_{s}\right)$, is a measurable space with nullity $(\mathscr{X}, \mathfrak{M}, \mathcal{N})$ defined as follows: $\mathscr{X}=$ $\bigcup_{s \in S}\left(\mathscr{X}_{s} \times\{s\}\right) ; \pi: \mathscr{X} \rightarrow \bigcup_{s \in S} \mathscr{X}_{s}$ is given by $\pi(x, s)=x ; A \in$ $\mathfrak{M}$ (respectively $A \in \mathcal{N})$ iff $\pi\left(A \cap\left(\mathscr{X}_{s} \times\{s\}\right)\right) \in \mathfrak{M}_{s}\left(\pi\left(A \cap\left(\mathscr{X}_{s} \times\right.\right.\right.$ $\{s\})) \in \mathcal{N}_{s}$ ) for every $s \in S$. Note that $\left\{\mathscr{X}_{s} \times\{s\}\right\}_{s \in S}$ is a base of $\bigoplus_{s \in S}\left(\mathscr{X}_{s}, \mathfrak{M}_{s}, \mathcal{N}_{s}\right)$. We call $\pi$ the canonical projection.

Let $(\mathscr{X}, \mathfrak{M}, \mathcal{N})$ be a multi-standard measurable space with nullity. Let $\mathscr{X}^{d}$ be the set of all points $x \in \mathscr{X}$ such that $\{x\} \notin \overline{\mathcal{N}}$. One may show that $\mathscr{X}^{d} \in \mathfrak{M}$ (since $\mathscr{X}$ is multi-standard), $\left.\mathfrak{M}\right|_{\mathscr{X}^{d}}$ is the power set of $\mathscr{X}^{d}$ and $\left.\mathcal{N}\right|_{\mathscr{X}^{d}}=\{\varnothing\}$. Points of $\mathscr{X}^{d}$ are called atoms, while $\mathscr{X}^{d}$ and its complement $\mathscr{X}^{c}$ are called, respectively, the discrete and continuous parts of $\mathscr{X}$. Further, if $(Y, \mathfrak{N}, \mu)$ is a nonatomic standard measure 
space, then there is $Z \in \mathcal{N}(\mu)$ such that $\left(Y \backslash Z,\left.\mathfrak{N}\right|_{Y \backslash Z},\left.\mathcal{N}(\mu)\right|_{Y \backslash Z}\right)$ is isomorphic to $\left([0,1], \mathfrak{B}([0,1]), \mathcal{L}_{0}\right)$ where $\mathcal{L}_{0}$ is the $\sigma$-ideal of all Borel subsets of $[0,1]$ whose Lebesgue measure is equal to 0 (by Theorem 14.3.9 on page 270 in [27]). Using this fact, one may check that there is a base of $(\mathscr{X}, \mathfrak{M}, \mathcal{N})$ whose every member either consists of a single point belonging to $\mathscr{X}^{d}$ or is isomorphic to $\left([0,1], \mathfrak{B}([0,1]), \mathcal{L}_{0}\right)$. Since every base of the last mentioned measurable space with nullity is countable (finite or not; to convince of that see the proof of Lemma 21.4 below), one deduces from this that either $\mathscr{X}^{c}$ is null or is a standard set, or every standard base of $(\mathscr{X}, \mathfrak{M}, \mathcal{N})$ contains the same, uncountable, number of sets almost isomorphic to $\left([0,1], \mathfrak{B}([0,1]), \mathcal{L}_{0}\right)$. We define two characteristic cardinal numbers related to $\mathscr{X}$ as follows: $\iota^{d}(\mathscr{X})=\operatorname{card}\left(\mathscr{X}^{d}\right)$ and $\iota^{c}(\mathscr{X})$ is either 0 (if $\mathscr{X}^{c}$ is null) or $\aleph_{0}$ (if $\mathscr{X}^{c}$ is standard), or is equal to the uncountable number of members of a standard base which are almost isomorphic to $\left([0,1], \mathfrak{B}([0,1]), \mathcal{L}_{0}\right)$. We see that two multi-standard measurable spaces with nullities $\mathscr{X}$ and $\mathscr{Y}$ are almost isomorphic iff $\iota^{d}(\mathscr{X})=\iota^{d}(\mathscr{Y})$ and $\iota^{c}(\mathscr{X})=\iota^{c}(\mathscr{Y})$. What is more, for any $\alpha \in$ Card and $\beta \in \operatorname{Card}_{\infty} \cup\{0\}$ there is a multistandard measurable space with nullity $\mathscr{Z}$ for which $\iota^{d}(\mathscr{Z})=\alpha$ and $\iota^{c}(\mathscr{Z})=\beta$. (Indeed, take a set $D$ of cardinality $\alpha$ and a set $S$ disjoint from $D$ whose cardinality is either $\beta$ if $\beta \neq \aleph_{0}$ or 1 if $\beta=\aleph_{0}$. For each $s \in S$ let $\left(I_{s}, \mathfrak{M}_{s}, \mathcal{N}_{s}\right)$ be a copy of $\left([0,1], \mathfrak{B}([0,1]), \mathcal{L}_{0}\right)$ and for $d \in D$ let $\left(I_{d}, \mathfrak{M}_{d}, \mathcal{N}_{d}\right)$ be a standard one-point measurable space with nullity. Now it suffices to define $\mathscr{Z}$ as $\bigoplus_{x \in D \cup S}\left(I_{x}, \mathfrak{M}_{x}, \mathcal{N}_{x}\right)$.)

From now on, $(\mathscr{X}, \mathfrak{M}, \mathcal{N})$ and $\left(\mathscr{X}^{\prime}, \mathfrak{M}^{\prime}, \mathcal{N}^{\prime}\right)$ denote multi-standard measurable spaces with nullities. Let $\Phi: \mathscr{X} \ni x \mapsto \mathbb{B}^{(x)} \in \mathcal{S} \mathcal{E} \mathcal{P}_{N}$ be any function. If there exist $Z \in \mathcal{N}$ and an integrable field $\mathscr{X} \backslash Z \ni$ $x \mapsto A^{(x)} \in \bigcup_{n=1}^{n=\infty} \operatorname{CDD}_{N}\left(\mathcal{H}_{n}\right)$ such that $\mathbb{A}^{(x)}=\Phi(x)$ for all $x \in \mathscr{X} \backslash Z$, we call $\Phi$ a summable field and define $\bigoplus_{x \in \mathscr{X}}^{\mathcal{N}} \mathbb{B}^{(x)}$ as follows. If $\mathscr{X}$ is trivial, we put $\bigoplus_{x \in \mathscr{X}}^{\mathcal{N}} \mathbb{B}^{(x)}=\mathbb{O}$. Otherwise let $\mathcal{B}$ be a standard base of $(\mathscr{X}, \mathfrak{M}, \mathcal{N})$. For every $B \in \mathcal{B}$ there is a standard measure $\mu_{B}$ on $\left(B,\left.\mathfrak{M}\right|_{B}\right)$ such that $\mathcal{N}\left(\mu_{B}\right)=\left.\mathcal{N}\right|_{B}$. We put

$$
\bigoplus_{x \in \mathscr{X}}^{\mathcal{N}} \mathbb{B}^{(x)}=\bigoplus_{B \in \mathcal{B}} \int_{B}^{\oplus} \mathbb{B}^{(x)} \mathrm{d} \mu_{B}(x) .
$$

The next result shows that $\bigoplus_{x \in \mathscr{X}}^{\mathcal{N}} \mathbb{B}^{(x)}$ is well defined.

21.3. Proposition. Formula (21-1) well defines $\bigoplus_{x \in \mathscr{X}}^{\mathcal{N}} \mathbb{B}^{(x)}$. That is, the right-hand side expression of (21-1) is independent of the choice of a standard base $\mathcal{B}$ and standard measures $\mu_{B}$ 's; and $\left\{\mathbb{B}^{(x)}\right\}_{x \in B}$ is an integrable (with respect to $\mu_{B}$ ) field for each $B \in \mathcal{B}$.

Proof. Let $\mathcal{B}_{1}$ and $\mathcal{B}_{2}$ be standard bases for $(\mathscr{X}, \mathfrak{M}, \mathcal{N})$ and $\left\{\mu_{B}^{(j)}: B \in\right.$ $\left.\mathcal{B}_{j}\right\}(j=1,2)$ corresponding families of standard measures. For each $D \in \mathcal{B}_{j}$ let $\left.D^{\prime} \in \mathfrak{M}\right|_{D}$ be such that $D \backslash D^{\prime} \in \mathcal{N}$ and $\left(D^{\prime},\left.\mathfrak{M}\right|_{D^{\prime}}\right)$ is a 
standard Borel space. Then the set

$$
I\left(D^{\prime}, \mathcal{B}_{3-j}\right)=\left\{E \in \mathcal{B}_{3-j}: D^{\prime} \cap E \neq \varnothing\right\} \quad \text { is countable }
$$

(see the last fragment of the proof of Lemma 21.4 below). Additionally put $\mathcal{I}=\left\{\left(D_{1}, D_{2}\right) \in \mathcal{B}_{1} \times \mathcal{B}_{2}: D_{1}^{\prime} \cap D_{2}^{\prime} \notin \mathcal{N}\right\}$. Thanks to (DI3) (page 77) and (21-2) we obtain

$$
\begin{aligned}
& \bigoplus_{A \in \mathcal{B}_{1}} \int_{A}^{\oplus} \mathbb{B}^{(x)} \mathrm{d} \mu_{A}^{(1)}(x)= \\
&=\bigoplus_{A \in \mathcal{B}_{1}}\left(\bigoplus\left\{\int_{A^{\prime} \cap B^{\prime}}^{\oplus} \mathbb{B}^{(x)} \mathrm{d} \mu_{A}^{(1)}(x):(A, B) \in \mathcal{I}\right\}\right)= \\
&=\bigoplus\left\{\int_{A^{\prime} \cap B^{\prime}}^{\oplus} \mathbb{B}^{(x)} \mathrm{d} \mu_{A}^{(1)}(x):(A, B) \in \mathcal{I}\right\}
\end{aligned}
$$

and similarly

$$
\bigoplus_{B \in \mathcal{B}_{2}} \int_{B}^{\oplus} \mathbb{B}^{(x)} \mathrm{d} \mu_{B}^{(2)}(x)=\bigoplus\left\{\int_{A^{\prime} \cap B^{\prime}}^{\oplus} \mathbb{B}^{(x)} \mathrm{d} \mu_{B}^{(2)}(x):(A, B) \in \mathcal{I}\right\} .
$$

Now the notice that $\mathcal{N}\left(\left.\mu_{A}^{(1)}\right|_{A^{\prime} \cap B^{\prime}}\right)=\mathcal{N}\left(\left.\mu_{B}^{(2)}\right|_{A^{\prime} \cap B^{\prime}}\right)$ combined with (DI4) (page 77) yields that

$$
\bigoplus_{A \in \mathcal{B}_{1}} \int_{A}^{\oplus} \mathbb{B}^{(x)} \mathrm{d} \mu_{A}^{(1)}(x)=\bigoplus_{B \in \mathcal{B}_{2}} \int_{B}^{\oplus} \mathbb{B}^{(x)} \mathrm{d} \mu_{B}^{(2)}(x) .
$$

The remainder is left for the reader.

It is easily seen that the restriction of a summable field to a measurable set is summable as well. Thanks to Proposition 21.3, we may rewrite (21-1) in a new form: whenever $\mathcal{B}$ is a standard base of $(\mathscr{X}, \mathfrak{M}, \mathcal{N})$ and $\left\{\mathbb{A}^{(x)}\right\}_{x \in \mathscr{X}}$ is summable,

$$
\bigoplus_{x \in \mathscr{X}}^{\mathcal{N}} \mathbb{A}^{(x)}=\bigoplus_{B \in \mathcal{B}}\left(\bigoplus_{x \in B}^{\mathcal{N}} \mathbb{A}^{(x)}\right)
$$

Using this, one may prove that (21-3) is satisfied for a completely arbitrary (unnecessarily standard) base $\mathcal{B}$.

Our next goal is to extend the notion of summability to more general context. In what follows, we equip $\mathbb{R}_{+} \cup$ Card with the Borel structure induced by the order topology (precisely, each of the sets $I_{\alpha}$ with $\alpha \in$ $\operatorname{Card}_{\infty}$ is equipped with this Borel structure).

21.4. Lemma. For a function $f: \mathscr{X} \rightarrow \mathbb{R}_{+} \cup$ Card the following conditions are equivalent:

(i) $f$ is almost measurable,

(ii) there is $Z \in \mathcal{N}$ with the following properties:
(a) $A=f^{-1}\left(\mathbb{R}_{+}\right) \backslash Z \in \mathfrak{M}$ and $\left.f\right|_{A}: A \rightarrow \mathbb{R}_{+}$is measurable,
(b) for every $\alpha \in \operatorname{Card}_{\infty}, f^{-1}(\{\alpha\}) \backslash Z \in \mathfrak{M}$, 
(c) for each standard set $B \in \mathfrak{M}$ there exists $Z_{B} \in \mathcal{N}$ such that the set $f\left(B \backslash Z_{B}\right) \cap \operatorname{Card}_{\infty}$ is countable (finite or not).

Proof. Suppose all conditions of (ii) are fulfilled. In what follows we preserve the notation of (ii). Let $\mathcal{B}$ be a standard base of $\mathscr{X}$. Put $\mathscr{Z}=Z \cup \bigcup_{B \in \mathcal{B}}\left(B \cap Z_{B}\right)$. Then $\mathscr{Z} \in \mathcal{N}$ and points (a)-(c) imply that $\left.f\right|_{\mathscr{X} \backslash \mathscr{Z}}$ is measurable.

Now assume that $Z \in \mathcal{N}$ is such that $\left.f\right|_{\mathscr{X} \backslash Z}$ is measurable. It is clear that conditions (a) and (b) are satisfied. To show (c), it suffices to prove the following claim: if $(Y, \mathfrak{N})$ is a standard Borel space and $u: Y \rightarrow I_{\gamma}$ is measurable, then $D=u(Y) \cap \operatorname{Card}_{\infty}$ is countable. Since $D$ is well ordered, $D$ is countable iff so is the subset $D_{0}$ of $D$ consisting of all elements of $D$ which have the direct precedessor (relative to $D$ ) in $D$. Note that if $\alpha \in D_{0}$, then $\{\alpha\}$ is open in $D$ with respect to the topology inherited from $I_{\gamma}$. Consequently, every subset of $D_{0}$ is open in $D$ and hence $Y_{0}=u^{-1}\left(D_{0}\right)$ is Borel and $\left.u\right|_{Y_{0}}$ is a Borel function of $Y_{0}$ (which is a standard Borel space) onto the discrete space $D_{0}$. It therefore follows from theory of Souslin sets that $D_{0}$ is countable. (Indeed, if $D_{0}$ was uncountable, there would exist a continuous mapping of $D_{0}$ onto a non-Souslin subset of $[0,1]$. It would then follow that a non-Souslin subset of $[0,1]$ could be the image of a standard Borel space under a Borel function, which is impossible.)

Lemma 21.4 has two important consequences: if $f, g: \mathscr{X} \rightarrow \mathbb{R}_{+} \cup$ Card are almost measurable and $\alpha \in$ Card, the functions $f+g$ and $\alpha \cdot f$ are almost measurable as well. We shall use these facts several times.

In the next two paragraphs $\Phi: \mathscr{D} \rightarrow \mathcal{S} \mathcal{E} \mathcal{P}_{N}$ is a summable field and $f: \mathscr{D} \rightarrow \mathbb{R}_{+} \cup$ Card is an almost measurable function where $\mathscr{D} \in \mathfrak{M}$ (notice that $\mathscr{D}$ is multi-standard).

We say that $f$ fits to $\Phi$ iff there are two disjoint measurable sets $D_{1}$ and $D_{2}$ such that $\mathscr{D} \backslash\left(D_{1} \cup D_{2}\right) \in \mathcal{N}, f\left(D_{1}\right) \subset$ Card and $\Phi\left(D_{2}\right) \subset \mathcal{S} \mathcal{M}_{N}$. (If $f$ fits to $\Phi, f(x) \odot \Phi(x)$ makes sense for almost all $x \in \mathscr{D}$.)

There is $Z \in \mathcal{N}$ such that the sets $A=f^{-1}\left(I_{\aleph_{0}} \backslash\{0\}\right) \backslash Z$ and $A_{\alpha}=$ $f^{-1}(\{\alpha\}) \backslash Z$ with uncountable $\alpha$ 's are measurable and the function $\left.f\right|_{A}: A \rightarrow I_{\aleph_{0}}$ is Borel. We call the pair $(f, \Phi)$ summable if $f$ fits to $\Phi$ and the field $A \ni x \mapsto f(x) \odot \Phi(x) \in \mathcal{S} \mathcal{E} \mathcal{P}_{N}$ is summable. If this is the case, we define $\bigoplus_{x \in \mathscr{D}}^{\mathcal{N}} f(x) \odot \Phi(x)$ by

$$
\bigoplus_{x \in \mathscr{D}}^{\mathcal{N}} f(x) \odot \Phi(x)=\left(\bigoplus_{x \in A}^{\mathcal{N}} f(x) \odot \Phi(x)\right) \oplus \bigoplus_{\alpha>\aleph_{0}}\left(\alpha \odot \bigoplus_{x \in A_{\alpha}}^{\mathcal{N}} \Phi(x)\right) .
$$

It is clear that summability of $(f, \Phi)$ and the formula for $\bigoplus_{x \in \mathscr{D}}^{\mathcal{N}} f(x) \odot$ $\Phi(x)$ is independent of the choice of $Z$. Notice that summability of $\Phi$ is equivalent to summability of $(\delta, \Phi)$ where $\delta: \mathscr{D} \rightarrow \mathbb{R}_{+} \cup$ Card is constantly equal to 1 . 
The following properties are infered from (DI0)-(DI4) (page 77) and (21-3). Everywhere below $\left(f,\left\{\mathbb{A}^{(x)}\right\}_{x \in \mathscr{X}}\right)$ is a summable pair.

(CS0) For each $\mathscr{D} \in \mathfrak{M}$ the pair $\left(f,\left\{\mathbb{A}^{(x)}\right\}_{x \in \mathscr{D}}\right)$ is summable as well and $\bigoplus_{x \in \mathscr{D}}^{\mathcal{N}} f(x) \odot \mathbb{A}^{(x)}=\mathbb{O}$ iff $s_{\mathscr{D}}(f):=\{x \in \mathscr{D}: \quad f(x) \neq 0\} \in \overline{\mathcal{N}}$ $\bigoplus_{x \in \mathscr{D}}^{\mathcal{N}} f(x) \odot \mathbb{A}^{(x)} \in \mathcal{S} \mathcal{E} \mathcal{P}_{N}$ iff there is $Z \in \mathcal{N}$ such that $s_{\mathscr{D}}(f) \backslash Z$ is standard.

(CS1) The pair $\left(f,\left\{\mathfrak{b}\left(\mathbb{A}^{(x)}\right)\right\}_{x \in \mathscr{X}}\right)$ is summable and $\mathfrak{b}\left(\bigoplus_{x \in \mathscr{X}}^{\mathcal{N}} f(x) \odot\right.$ $\left.\mathbb{A}^{(x)}\right)=\bigoplus_{x \in \mathscr{X}}^{\mathcal{N}} f(x) \odot \mathfrak{b}\left(\mathbb{A}^{(x)}\right)$.

(CS2) Whenever $\mathcal{B}$ is a base of $(\mathscr{X}, \mathfrak{M}, \mathcal{N})$,

$$
\bigoplus_{x \in \mathscr{X}}^{\mathcal{N}} f(x) \odot \mathbb{A}^{(x)}=\bigoplus_{B \in \mathcal{B}}\left(\bigoplus_{x \in B}^{\mathcal{N}} f(x) \odot \mathbb{A}^{(x)}\right) .
$$

(CS3) (A) If $\left(f,\left\{\mathbb{B}^{(x)}\right\}_{x \in \mathscr{X}}\right)$ is summable, so is $\left(f,\left\{\mathbb{A}^{(x)} \oplus \mathbb{B}^{(x)}\right\}_{x \in \mathscr{X}}\right)$ and

$$
\bigoplus_{x \in \mathscr{X}}^{\mathcal{N}} f(x) \odot\left(\mathbb{A}^{(x)} \oplus \mathbb{B}^{(x)}\right)=\left(\bigoplus_{x \in \mathscr{X}}^{\mathcal{N}} f(x) \odot \mathbb{A}^{(x)}\right) \oplus\left(\bigoplus_{x \in \mathscr{X}}^{\mathcal{N}} f(x) \odot \mathbb{B}^{(x)}\right)
$$

(B) For every $\alpha \in$ Card, the pair $\left(\alpha \cdot f,\left\{\mathbb{A}^{(x)}\right\}_{x \in \mathscr{X}}\right)$ is summable and $\bigoplus_{x \in \mathscr{X}}^{\mathcal{N}}(\alpha \cdot f(x)) \odot \mathbb{A}^{(x)}=\alpha \odot\left(\bigoplus_{x \in \mathscr{X}}^{\mathcal{N}} f(x) \odot \mathbb{A}^{(x)}\right)$.

(C) If in addition $\left(g,\left\{\mathbb{A}^{(x)}\right\}_{x \in \mathscr{K}}\right)$ is summable, so is the pair $\left(f+g,\left\{\mathbb{A}^{(x)}\right\}_{x \in \mathscr{X}}\right)$ and

$\bigoplus_{x \in \mathscr{X}}^{\mathcal{N}}(f(x)+g(x)) \odot \mathbb{A}^{(x)}=\left(\bigoplus_{x \in \mathscr{X}}^{\mathcal{N}} f(x) \odot \mathbb{A}^{(x)}\right) \oplus\left(\bigoplus_{x \in \mathscr{X}}^{\mathcal{N}} g(x) \odot \mathbb{A}^{(x)}\right)$.

(CS4) If $\psi: \mathscr{X}^{\prime} \rightarrow \mathscr{X}$ is an almost null-isomorphism, the pair ( $f \circ$ $\left.\psi,\left\{\mathbb{A}^{\left(\psi\left(x^{\prime}\right)\right)}\right\}_{x^{\prime} \in \mathscr{X}^{\prime}}\right)$ is summable and $\bigoplus_{x^{\prime} \in \mathscr{X}^{\prime}}^{\mathcal{N}^{\prime}} f\left(\psi\left(x^{\prime}\right)\right) \odot \mathbb{A}^{\left(\psi\left(x^{\prime}\right)\right)}=$ $\bigoplus_{x \in \mathscr{X}}^{\mathcal{N}} f(x) \odot \mathbb{A}^{(x)}$.

Since properties (CS3)-(B) and (CS3)-(C) are of great importance for us and are not so easy, let us prove them. It is quite simple that both the pairs appearing in the assertions of these points are summable. Thanks to (CS2), we may assume that $\mathscr{X}$ is standard. It then follows from Lemma 21.4 that we may also assume both the sets $f(\mathscr{X}) \cap \operatorname{Card}_{\infty}$ and $g(\mathscr{X}) \cap \operatorname{Card}_{\infty}$ are countable and $f$ and $g$ are Borel. We start with (CS3)-(B). Observe that (DI3) yields the assertion for $\alpha \leqslant \aleph_{0}$. So, $\aleph_{0} \odot\left(\bigoplus_{x \in \mathscr{X}}^{\mathcal{N}} f(x) \odot \mathbb{A}^{(x)}\right)=\bigoplus_{x \in \mathscr{X}}^{\mathcal{N}}\left(\aleph_{0} \cdot f(x)\right) \odot \mathbb{A}^{(x)}$. This implies that we may further assume that $f(\mathscr{X}) \subset \operatorname{Card}_{\infty}$ (replacing $f$ by $\aleph_{0} \cdot f$ and reducing $\mathscr{X}$ to $\left.s(f)=s_{\mathscr{X}}(f)\right)$. But then the assertion easily follows from (CS2) and the countability of $f(\mathscr{X})$.

We now pass to $(\mathrm{CS} 3)-(\mathrm{C})$. Put $A_{f}\left(\aleph_{0}\right)=f^{-1}\left(I_{\aleph_{0}}\right)$ and $A_{f}(\alpha)=$ $f^{-1}(\{\alpha\})$ for uncountable $\alpha$. In the same way define the sets $A_{g}(\beta)$ (corresponding to $g$ ) for $\beta \in \operatorname{Card}_{\infty}$. Notice that the sets $I_{f}=\{\alpha \in$ $\left.\operatorname{Card}_{\infty}: A_{f}(\alpha) \neq \varnothing\right\}$ and $I_{g}=\left\{\alpha \in \operatorname{Card}_{\infty}: A_{g}(\alpha) \neq \varnothing\right\}$ are countable and hence the family $\left\{A_{f}(\alpha) \cap A_{g}(\beta):(\alpha, \beta) \in I_{f} \times I_{g}\right\}$ is a base of $(\mathscr{X}, \mathfrak{M}, \mathcal{N})$. Using again $(\mathrm{CS} 2)$, we may therefore assume that $I_{f}$ 
and $I_{g}$ consist of single cardinals. The case $I_{f}=I_{g}=\left\{\aleph_{0}\right\}$ follows from (DI3), while the one when $\aleph_{0} \notin I_{f} \cup I_{g}$ is obvious. Finally, if e.g. $I_{f}=\left\{\aleph_{0}\right\}$ and $I_{g}=\{\alpha\}$ for some $\alpha>\aleph_{0}$, then (by (CS3)-(B)) $\bigoplus_{x \in \mathscr{X}}^{\mathcal{N}}(f(x)+g(x)) \odot \mathbb{A}^{(x)}=\bigoplus_{x \in \mathscr{X}}^{\mathcal{N}} g(x) \odot \mathbb{A}^{(x)}=\alpha \odot \bigoplus_{x \in \mathscr{X}}^{\mathcal{N}} \mathbb{A}^{(x)} \geqslant$ $\bigoplus_{x \in \mathscr{X}}^{\mathcal{N}} \aleph_{0} \odot \mathbb{A}^{(x)}$ and (again by (CS2) and (CS3)-(B))

$$
\begin{aligned}
\bigoplus_{x \in \mathscr{X}}^{\mathcal{N}} \aleph_{0} \odot \mathbb{A}^{(x)}= & \left(\bigoplus_{x \in \mathscr{X}}^{\mathcal{N}}\left(\aleph_{0} \cdot f(x)\right) \odot \mathbb{A}^{(x)}\right) \oplus\left(\bigoplus_{x \notin s(f)}^{\mathcal{N}} \aleph_{0} \odot \mathbb{A}^{(x)}\right) \\
& \geqslant \aleph_{0} \odot\left(\bigoplus_{x \in \mathscr{X}}^{\mathcal{N}} f(x) \odot \mathbb{A}^{(x)}\right) \geqslant \bigoplus_{x \in \mathscr{X}}^{\mathcal{N}} f(x) \odot \mathbb{A}^{(x)}
\end{aligned}
$$

which finishes the proof.

We now repeat the idea of the previous section. Let $\left(f,\left\{\mathbb{A}^{(x)}\right\}_{x \in \mathscr{X}}\right)$ be a summable pair. If

$$
\bigoplus_{x \in \mathscr{D}^{\prime}}^{\mathcal{N}} f(x) \odot \mathbb{A}^{(x)} \perp_{u} \bigoplus_{x \in \mathscr{D}^{\prime \prime}}^{\mathcal{N}} f(x) \odot \mathbb{A}^{(x)}
$$

for any two disjoint sets $\mathscr{D}^{\prime}, \mathscr{D}^{\prime \prime} \in \mathfrak{M}$, we call the pair $\left(f,\left\{\mathbb{A}^{(x)}\right\}_{x \in \mathscr{X}}\right)$ regular and we write $\bigoplus_{x \in \mathscr{X}}^{\mathcal{N}} f(x) \odot \mathbb{A}^{(x)}$ in place of $\bigoplus_{x \in \mathscr{X}}^{\mathcal{N}} f(x) \odot \mathbb{A}^{(x)}$.

Similarly, a summable field $\left\{\mathbb{A}^{(x)}\right\}_{x \in \mathscr{X}}$ is regular iff (21-4) if fulfilled with $f$ constantly equal to 1 . As usual, the usage of $\boxplus_{x \in \mathscr{X}}^{\mathcal{N}} f(x) \odot$ $\mathbb{A}^{(x)}$ includes information that $\left(f,\left\{\mathbb{A}^{(x)}\right\}_{x \in \mathscr{K}}\right)$ is regular. Note that, by definition, regular pairs and fields are summable.

The next result collects fundamental facts on the just defined notion.

21.5. Theorem. Let $\Phi: \mathscr{X} \ni x \rightarrow \mathbb{A}^{(x)} \in \mathfrak{F}_{N}$ be any function.

(I) The following conditions are equivalent:

(i) the field $\left\{\mathbb{A}^{(x)}\right\}_{x \in \mathscr{X}}$ is regular,

(ii) for every standard set $A \in \mathfrak{M}$ there is $Z \in \mathcal{N}$ such that $\Phi(A \backslash Z)$ is a measurable domain and $\left.\Phi\right|_{A \backslash Z}$ is a Borel isomorphism of $A \backslash Z$ onto $\Phi(A \backslash Z)$.

(II) If $\Phi$ satisfies condition (ii) of point (I) and $f: \mathscr{X} \rightarrow \mathbb{R}_{+} \cup$ Card is an almost measurable function which fits to $\Phi$, then $\left(f,\left\{\mathbb{A}^{(x)}\right\}_{x \in \mathscr{X}}\right)$ is regular. Moreover,

$$
\begin{aligned}
\left\{\mathbb{Y} \in \mathcal{C D D}_{N}: \mathbb{Y} \leqslant s \bigoplus_{x \in \mathscr{X}}^{\mathcal{N}}\right. & \left.f(x) \odot \mathbb{A}^{(x)}\right\}= \\
& =\left\{\bigoplus_{x \in \mathscr{D}}^{\mathcal{N}} f(x) \odot \mathbb{A}^{(x)}: \mathscr{D} \in \mathfrak{M}\right\} .
\end{aligned}
$$

Proof. Implication '(i) $\Longrightarrow$ (ii)' in point (I) follows immediately from Proposition 20.4 (page 78). To prove the converse one, first note that $\Phi$ is summable because of (ii), the existence of a standard base of $\mathscr{X}$ and Proposition 20.4. Further, take two disjoint nonnull measurable 
sets $\mathscr{D}_{1}$ and $\mathscr{D}_{2}$. Let $\mathcal{B}_{j}$ be a standard base of $\mathscr{D}_{j}$. Since $B_{1} \cup B_{2}$ is standard for $B_{j} \in \mathscr{D}_{j}$, we infer from point (ii) and Proposition 20.4 that $\bigoplus_{x \in B_{1}}^{\mathcal{N}} \mathbb{A}^{(x)} \perp_{u} \bigoplus_{x \in B_{2}}^{\mathcal{N}} \mathbb{A}^{(x)}$. Consequently, $\bigoplus_{B \in \mathcal{B}_{1}}\left(\bigoplus_{x \in B}^{\mathcal{N}} \mathbb{A}^{(x)}\right) \perp_{u}$ $\bigoplus_{B \in \mathcal{B}_{2}}\left(\bigoplus_{x \in B}^{\mathcal{N}} \mathbb{A}^{(x)}\right)$ and hence the assertion of (i) follows from (CS2).

Now assume $\Phi$ and $f$ are as in (II). We may assume that $f$ is Borel. Define $f_{0}: \mathscr{X} \rightarrow I_{\aleph_{0}} \backslash\{0\}$ by $f_{0}(x)=f(x)$ if $f(x) \in I_{\aleph_{0}} \backslash\{0\}$ and $f_{0}(x)=1$ otherwise. The function $f_{0}$ is Borel and fits to $\Phi$. Let $B \in \mathfrak{M}$ be a standard set. Then there is a standard measure $\mu$ on $\left(B,\left.\mathfrak{M}\right|_{B}\right)$ such that $\mathcal{N}(\mu)=\left.\mathcal{N}\right|_{B}$. We infer from the assumptions that $\left(\mu,\left.\Phi\right|_{B}\right) \in \operatorname{RGS}\left(B,\left.\mathfrak{M}\right|_{B}\right)$. Hence, Lemma 20.8 (page 82) implies that

$$
\left(\mu,\left.\left(f_{0} \odot \Phi\right)\right|_{B}\right) \in \operatorname{RGS}\left(B,\left.\mathfrak{M}\right|_{B}\right) .
$$

Consequently, if $B_{1}$ and $B_{2}$ are two disjoint standard (measurable) subsets of $\mathscr{X}$, then

$$
\bigoplus_{x \in B_{1}}^{\mathcal{N}} f_{0}(x) \odot \mathbb{A}^{(x)} \perp_{u} \bigoplus_{x \in B_{2}}^{\mathcal{N}} f_{0}(x) \odot \mathbb{A}^{(x)}
$$

We also conclude from (21-6) that $\left(f_{0}, \Phi\right)$ is summable on every standard subset of $\mathscr{X}$. Since $\mathscr{X}$ is multi-standard, $\left(f_{0}, \Phi\right)$ is therefore summable. It now follows from the definitions of $f_{0}$ and of summability that $(f, \Phi)$ is summable as well.

Further, if $B$ is a standard subset of $\mathscr{X}$, it follows from Lemma 21.4 and the definitions of $f_{0}$ and of $\bigoplus_{x \in B}^{\mathcal{N}} f(x) \odot \Phi(x)$ that $\bigoplus_{x \in B}^{\mathcal{N}} f(x) \odot$ $\Phi(x) \ll \bigoplus_{x \in B}^{\mathcal{N}} f_{0}(x) \odot \mathbb{A}^{(x)}$. This combined with (21-7) yields that

$$
\bigoplus_{x \in B_{1}}^{\mathcal{N}} f(x) \odot \mathbb{A}^{(x)} \perp_{u} \bigoplus_{x \in B_{2}}^{\mathcal{N}} f(x) \odot \mathbb{A}^{(x)}
$$

for any two disjoint standard sets $B_{1}, B_{2} \subset \mathscr{X}$. Now if $\mathscr{D}^{\prime}$ and $\mathscr{D}^{\prime \prime}$ are two arbitrary disjoint nonnull Borel subsets of $\mathscr{X}$, the fact that they are multi-standard together with (CS2) and (21-8) gives (21-4). It therefore suffices to check (21-5). We have already shown the inclusion ' $\supset$ ' in (21-5) (cf. (CS2)). To this end, fix $\mathbb{Y} \in \mathcal{C D D}_{N}$ such that

$$
\mathbb{Y} \leqslant s \bigoplus_{x \in \mathscr{X}}^{\mathcal{N}} f(x) \odot \mathbb{A}^{(x)}
$$

Let $\mathcal{B}_{0}$ be a standard base of $\mathscr{X}$. Thanks to Lemma 21.4, for every $B \in \mathcal{B}_{0}$ there are pairwise disjoint measurable subsets $W_{0}^{B}, W_{1}^{B}, \ldots$ of $B$ such that $B \backslash \bigcup_{n=0}^{\infty} W_{n}^{B} \in \mathcal{N}, f\left(W_{0}^{B}\right) \subset \mathbb{R}_{+} \backslash\{0\}$ and $\left.f\right|_{W_{n}^{B}}$ is constantly equal to some $\alpha \in \operatorname{Card}_{\infty} \cup\{0\}$. Notice that then $\mathcal{B}^{n}=$ $\left\{W_{n}^{B}: B \in \mathcal{B}_{0}, n \geqslant 0\right\} \backslash \mathcal{N}$ is a standard base of $\mathscr{X}$ as well. Denote by $\mathcal{B}_{f}$ the set of all $B \in \mathcal{B}$ for which $f(B) \subset \mathbb{R}_{+} \backslash\{0\}$ and let $\mathcal{B}^{\prime}=$ $\mathcal{B} \backslash \mathcal{B}_{f}$. For each $B \in \mathcal{B}^{\prime}$ there is (unique) $\alpha_{B} \in \operatorname{Card}_{\infty} \cup\{0\}$ such that 
$f(B)=\left\{\alpha_{B}\right\}$. Now (CS2), (CS3) and already proved part of (II) give

$$
\begin{aligned}
& \bigoplus_{x \in \mathscr{X}}^{\mathcal{N}} f(x) \odot \mathbb{A}^{(x)}= \\
& \quad=\left[\bigoplus_{B \in \mathcal{B}_{f}}\left(\bigoplus_{x \in B}^{\mathcal{N}} f(x) \odot \mathbb{A}^{(x)}\right)\right] \boxplus\left[\bigoplus_{B \in \mathcal{B}^{\prime}} \alpha_{B} \odot\left(\bigoplus_{x \in B}^{\mathcal{N}} \mathbb{A}^{(x)}\right)\right] .
\end{aligned}
$$

It may be deduced from (21-9) and (21-10) (using e.g. Proposition 6.4, page 15, and Theorem 6.1, page 13) that $\mathbb{Y}$ is of the form

$$
\mathbb{Y}=\left(\bigoplus_{B \in \mathcal{B}_{f}} \mathbb{Y}_{B}\right) \boxplus\left(\bigoplus_{B \in \mathcal{B}^{\prime}} \widetilde{\mathbb{Y}}_{B}\right)
$$

where $\mathbb{Y}_{B} \leqslant^{s} \boxplus_{x \in B}^{\mathcal{N}} f(x) \odot \mathbb{A}^{(x)}$ for $B \in \mathcal{B}_{f}$ and $\widetilde{\mathbb{Y}}_{B} \leqslant^{s} \alpha_{B} \odot \boxplus_{x \in B}^{\mathcal{N}} \mathbb{A}^{(x)}$ for $B \in \mathcal{B}^{\prime}$. Further, by (PR6) (page 9), for each $B \in \mathcal{B}^{\prime}$ there is $\mathbb{Y}_{B} \leqslant s$ $\boxplus_{x \in B}^{\mathcal{N}} \mathbb{A}^{(x)}$ such that $\widetilde{\mathbb{Y}}_{B}=\alpha_{B} \odot \mathbb{Y}_{B}$. Since $\mathcal{B}$ consists of standard sets, we infer from Proposition 20.6 (page 80) that for every $B \in \mathcal{B}$ there exists a measurable set $\mathscr{D}_{B} \subset B$ for which $\mathbb{Y}_{B}=\boxplus_{x \in \mathscr{D}_{B}}^{\mathcal{N}} f(x) \odot$ $\mathbb{A}^{(x)}$ provided $B \in \mathcal{B}_{f}$ and $\mathbb{Y}_{B}=\boxplus_{x \in \mathscr{D}_{B}}^{\mathcal{N}} \mathbb{A}^{(x)}$ if $B \in \mathcal{B}^{\prime}$. Put $\mathscr{D}=$ $\bigcup_{B \in \mathcal{B}} \mathscr{D}_{B}$ and note that $\mathscr{D}$ is Borel since $\mathcal{B}$ is a base. Finally, the family $\left\{\mathscr{D}_{B}: B \in \mathcal{B}\right\}$ is a base of $\mathscr{D}$ and hence we obtain from (CS2) and (CS3) that

$$
\begin{aligned}
\bigoplus_{x \in \mathscr{D}}^{\mathcal{N}} f(x) \odot \mathbb{A}^{(x)}=\bigoplus_{B \in \mathcal{B}}\left(\bigoplus_{x \in \mathscr{D}_{B}}^{\mathcal{N}} f(x) \odot \mathbb{A}^{(x)}\right)= \\
=\left(\bigoplus_{B \in \mathcal{B}_{f}} \mathbb{Y}_{B}\right) \boxplus\left(\prod_{B \in \mathcal{B}^{\prime}} \alpha_{B} \odot \mathbb{Y}_{B}\right)=\mathbb{Y}
\end{aligned}
$$

and we are done.

Similarly as in the previous section, for a field $\Phi: \mathscr{X} \rightarrow \mathfrak{F}_{N}$ we shall write $\Phi \in \mathrm{RGS}_{l o c}$ or $\Phi \in \operatorname{RGS}_{l o c}(\mathscr{X})$ if $\Phi$ satisfies condition (ii) of Theorem 21.5.

\section{PRime DeCOMposition}

Semiprimes are those members of $\mathfrak{p}_{N}$ which make the issue of prime decomposition of $N$-tuples more complicated and ambiguous. To shape this in a way similar to that in the ring of natural numbers, we have to allow multiplicity functions to take real values (beside infinite cardinals) instead of (only) integer ones. Such an approach is therefore similar to Ernest's multiplicity theory (Chapter 4 of [9]) and will enable us to propose the prime decomposition of an arbitrary $N$-tuple in an (essentially) unique form (see Theorem 22.14, page 104). We consider this as a more attractive manner of 'factor decomposing' of $N$-tuples than Ernest's central decomposition [9]. 
In this section $(\mathscr{X}, \Phi)$ is a fixed pair such that $(\mathscr{X}, \mathfrak{M}, \mathcal{N})$ is a multistandard measurable space with nullity and $\Phi \in \operatorname{RGS}_{l o c}(\mathscr{X})$ is such that $\Phi(\mathscr{X}) \subset \mathfrak{p}_{N}$. After erasing from $\mathscr{X}$ a null measurable set, we may assume $\Phi$ is measurable. Let

$$
\mathscr{X}_{I}=\Phi^{-1}\left(\mathfrak{a}_{N}\right), \quad \mathscr{X}_{I I}=\Phi^{-1}\left(\mathfrak{s}_{N}\right), \quad \mathscr{X}_{I I I}=\Phi^{-1}\left(\mathfrak{f}_{N}\right) .
$$

Notice that $\mathscr{X}_{I}, \mathscr{X}_{I I}$ and $\mathscr{X}_{I I I}$ are measurable, pairwise disjoint and $\mathscr{X}_{I} \cup \mathscr{X}_{I I} \cup \mathscr{X}_{I I I}=\mathscr{X}$.

22.1. Definition. A function $f: \mathscr{D} \rightarrow \mathbb{R}_{+} \cup$ Card where $\mathscr{D} \in \mathfrak{M}$ is admissible for $\Phi$ iff $f$ is almost measurable, $f\left(\mathscr{X}_{I} \cap \mathscr{D}\right) \subset$ Card and $f\left(\mathscr{X}_{I I I} \cap \mathscr{D}\right) \subset\{0\} \cup \operatorname{Card}_{\infty}$. The class of all admissible functions on $\mathscr{X}$ is denoted by $\mathscr{A}(\mathscr{X}, \Phi)$ or shortly by $\mathscr{A}(\mathscr{X})$.

For each $f \in \mathscr{A}(\mathscr{X}), s(f)$ is the support of $f$, i.e. $s(f)=\{x \in$ $\mathscr{X}: f(x) \neq 0\}(s(f)$ is measurable provided so is $f)$.

Note that each admissible function fits to $\Phi$. Thus, by Theorem 21.5 (page 92), for every $f \in \mathscr{A}(\mathscr{X})$ we may write $\boxplus_{x \in \mathscr{X}}^{\mathcal{N}} f(x) \odot \Phi(x)$. As it is practised in measure theory, the term almost everywhere, which we shall abbreviate by writing a.e., will mean that suitable property (relation, etc.) holds true on $\mathscr{X} \backslash Z$ for some $Z \in \overline{\mathcal{N}}$.

As a consequence of Lemma 21.4 (page 89) we obtain

22.2. Corollary. For $f, g \in \mathscr{A}(\mathscr{X})$,

(a) $f+g, f \cdot g, f \vee g, f \wedge g \in \mathscr{A}(\mathscr{X})$ where $f \vee g=\max (f, g)$ and $f \wedge g=\min (f, g)$

(b) $\alpha \cdot f \in \mathscr{A}(\mathscr{X})$ for each $\alpha \in$ Card,

(c) if $f\left(\mathscr{X}_{I} \cup \mathscr{X}_{I I I}\right) \subset\{0\}, t \cdot f \in \mathscr{A}(\mathscr{X})$ for every $t \in \mathbb{R}_{+}$,

(d) if $f \leqslant g$ a.e., there is $u \in \mathscr{A}(\mathscr{X})$ such that $g=f+u$ a.e.

We leave the proof of Corollary 22.2 as an exercise. A part of it may be strengthened:

22.3. Lemma. Whenever $f_{1}, f_{2}, \ldots$ are admissible functions, so are $\bigwedge_{n \geqslant 1} f_{n}: \mathscr{X} \ni x \mapsto \inf _{n \geqslant 1} f_{n}(x) \in \mathbb{R}_{+} \cup$ Card and $\bigvee_{n \geqslant 1} f_{n}: \mathscr{X} \ni x \mapsto$ $\sup _{n \geqslant 1} f_{n}(x) \in \mathbb{R}_{+} \cup$ Card. In particular, $\sum_{n=1}^{\infty} f_{n} \in \mathscr{A}(\mathscr{X})$ (where $\left.\left(\sum_{n=1}^{\infty} f_{n}\right)(x)=\sum_{n=1}^{\infty} f_{n}(x)\right)$.

Proof. We leave this as an exercise that it is enough to show, thanks to Lemma 21.4 (page 89), that the closure of any countable subset $K$ of $\operatorname{Card}_{\infty}$ (in $I_{\gamma} \supset K$ whatever $\gamma \in \operatorname{Card}_{\infty}$ is) is countable as well (recall that countable compact Hausdorff spaces are metrizable, by $[9$, Theorem 3.1.9]). But this is quite simple: for every non-last element $x$ of $L=(\operatorname{cl} K) \backslash K$ there exists $c_{x} \in K$ which lies between $x$ and its direct successor (relative to $L$ ) in $L$. Since the function $L \ni x \mapsto c_{x} \in K$ is one-to-one, the assertion follows. 
22.4. Proposition. For $f, g \in \mathscr{A}(\mathscr{X})$,

$$
\bigoplus_{x \in \mathscr{X}}^{\mathcal{N}} f(x) \odot \Phi(x)=\bigoplus_{x \in \mathscr{X}}^{\mathcal{N}} g(x) \odot \Phi(x)
$$

iff $f=g$ a.e.

Proof. The 'if' part is clear. Suppose (22-1) holds true. It follows from (CS3) (page 91) that $\boxplus_{x \in \mathscr{B}}^{\mathcal{N}} u(x) \odot \Phi(x) \ll \bigoplus_{x \in \mathscr{B}}^{\mathcal{N}} \Phi(x)$ for each $\mathscr{B} \in \mathfrak{M}$ and $u \in\{f, g\}$. Since $\boxplus_{x \in \mathscr{B}}^{\mathcal{N}} \Phi(x) \perp_{u} \boxplus_{x \notin \mathscr{B}}^{\mathcal{N}} \Phi(x),(22-1)$ and (CS2) imply therefore that

$$
\bigoplus_{x \in \mathscr{B}}^{\mathcal{N}} f(x) \odot \Phi(x)=\bigoplus_{x \in \mathscr{B}}^{\mathcal{N}} g(x) \odot \Phi(x)
$$

for any $\mathscr{B} \in \mathfrak{M}$. Let $\mathscr{D} \in \mathfrak{M}$ be standard. It suffices to check that $f=g$ almost everywhere on $\mathscr{D}$. Thanks to Lemma 21.4 (page 89) we may assume that $\left.f\right|_{\mathscr{D}}$ and $\left.g\right|_{\mathscr{D}}$ are Borel and

$$
(f(\mathscr{D}) \cup g(\mathscr{D})) \cap \operatorname{Card}_{\infty} \text { is countable. }
$$

By $(22-3)$, the sets $\mathscr{D}_{+}=\{x \in \mathscr{D}: \quad f(x)<g(x)\}$ and $\mathscr{D}_{-}=\{x \in$ $\mathscr{D}: f(x)>g(x)\}$ are Borel. Suppose, for the contrary, that e.g. $\mathscr{D}_{+} \notin$ $\mathcal{N}$. We distinguish between two cases.

Assume there are a nonnull measurable set $\mathscr{B} \subset \mathscr{D}_{+}$and two cardinals $\alpha$ and $\beta$ such that $f(\mathscr{B})=\{\alpha\}$ and $g(\mathscr{B})=\{\beta\}$. Let $\mathbb{B}=$ $\boxplus_{x \in \mathscr{B}}^{\mathcal{N}} \Phi(x)$. We infer from (CS0) that $\mathbb{B} \neq \mathbb{O}$. Moreover, since $\Phi(\mathscr{X}) \subset \mathfrak{p}_{N}, \Phi \in \mathrm{RGS}_{l o c}$ and $\mathscr{B}$ is standard, Lemma 20.9 (page 83) yields that $\mathbb{B}$ is the direct sum of a minimal $N$-tuple and a semiminimal one. Consequently, $\alpha \odot \mathbb{B}<\beta \odot \mathbb{B}$ (to convince of that use e.g. Theorem 11.1, page 26, and (AO4), page 29, if needed). But this denies $(22-2)$ because $\boxplus_{x \in \mathscr{B}}^{\mathcal{N}} f(x) \odot \Phi(x)=\alpha \odot \mathbb{B}$ and $\boxplus_{x \in \mathscr{B}}^{\mathcal{N}} g(x) \odot \Phi(x)=$ $\beta \odot \mathbb{B}$.

Finally, if there is no set $\mathscr{B}$ with all above mentioned properties, it may be deduced from (22-3) that there exists a nonnull measurable set $\mathscr{B} \subset \mathscr{D}_{+} \cap \mathscr{X}_{I I}$ such that $f(\mathscr{B}) \subset \mathbb{R}_{+}$. Let $\mathbb{B}=\boxplus_{x \in \mathscr{B}}^{\mathcal{N}} f(x) \odot \Phi(x)$. As before, an application of Lemma 20.9 shows that

$$
\mathbb{B} \in \operatorname{S\mathcal {M}}_{N}
$$

On the other hand, there is a measurable function $u: \mathscr{B} \rightarrow\left(\mathbb{R}_{+} \cup\right.$ Card $) \backslash\{0\}$ such that $g(x)=f(x)+u(x)$ for all $x \in \mathscr{B}$. Then (CS3) combined with (22-2) gives $\mathbb{B}=\boxplus_{x \in \mathscr{B}}^{\mathcal{N}} g(x) \odot \Phi(x)=\mathbb{B} \oplus\left(\boxplus_{x \in \mathscr{B}}^{\mathcal{N}} u(x) \odot\right.$ $\Phi(x))$ which means, thanks to $(22-4)$, that $\boxplus_{x \in \mathscr{B}}^{\mathcal{N}} u(x) \odot \Phi(x)=\mathbb{O}$ (cf. $(\mathrm{AO} 4))$, contradictory to (CS0).

22.5. Theorem. Let $\mathbb{T}=\bigoplus_{x \in \mathscr{X}}^{\mathcal{N}} \Phi(x)$. Then

$$
\left\{\bigoplus_{x \in \mathscr{X}}^{\mathcal{N}} f(x) \odot \Phi(x): f \in \mathscr{A}(\mathscr{X}, \Phi)\right\}=\left\{\mathbb{X} \in \mathcal{C D} \mathcal{D}_{N}: \mathbb{X} \ll \mathbb{T}\right\}
$$


Proof. It easily follows from (CS3) (page 91) that $\boxplus_{x \in \mathscr{X}}^{\mathcal{N}} f(x) \odot \Phi(x) \ll$ $\mathbb{T}$ for every $f \in \mathscr{A}(\mathscr{X})$. We fix $\mathbb{X} \in \mathcal{C D} \mathcal{D}_{N}$ such that $\mathbb{X} \ll \mathbb{T}$. Let $\left\{B_{s}\right\}_{s \in S}$ be a standard base of $\mathscr{X}$. We may assume that $\bigcup_{s \in S} B_{s}=\mathscr{X}$. For each $s \in S$ put $\mathbb{T}_{s}=\boxplus_{x \in B_{s}}^{\mathcal{N}} \Phi(x)$. We infer from (CS0) that $\mathbb{T}_{s} \in \mathcal{S E \mathcal { P }} \mathcal{N}_{N}$ and from (CS2) that $\mathbb{T}=\boxplus_{s \in S} \mathbb{T}_{s}$. Let $\mathbb{X}_{s}=\mathbb{E}\left(\mathbb{X} \mid \mathbb{T}_{s}\right)$. Observe that $\mathbb{X}=\boxplus_{s \in S} \mathbb{X}_{s}$ and $\mathbb{X}_{s} \ll \mathbb{T}_{s}$. Suppose for each $s \in S$ there is an admissible function $f_{s}: B_{s} \rightarrow \mathbb{R}_{+} \cup$ Card such that $\mathbb{X}_{s}=$ $\boxplus_{x \in B_{s}}^{\mathcal{N}} f_{s}(x) \odot \Phi(x)$. Then the union $f: \mathscr{X} \rightarrow \mathbb{R}_{+} \cup$ Card of $f_{s}$ 's is admissible as well and it follows from (CS2) that

$$
\bigoplus_{x \in \mathscr{X}}^{\mathcal{N}} f(x) \odot \Phi(x)=\bigoplus_{s \in S}\left(\bigoplus_{x \in B_{s}}^{\mathcal{N}} f_{s}(x) \odot \Phi(x)\right)=\bigoplus_{s \in S} \mathbb{X}_{s}=\mathbb{X}
$$

The above argument reduces the problem to the case when $\mathscr{X}$ is standard. Then there is a standard measure $\mu$ on $\mathfrak{M}$ such that $\mathcal{N}(\mu)=\mathcal{N}$. Consequently,

$$
\bigoplus_{x \in \mathscr{X}}^{\mathcal{N}} f(x) \odot \Phi(x)=\int_{\mathscr{X}}^{\boxplus} f(x) \odot \Phi(x) \mathrm{d} \mu(x)
$$

for every Borel function $f: \mathscr{X} \rightarrow I_{\aleph_{0}}$ which fits to $\Phi$. Recall that for each $\mathbb{A} \in \mathcal{C D D}_{N}, s(\mathbb{A})$ is given by (15-5) (page 46) and $s(\mathbb{A})=\bigwedge\{\mathbb{E} \leqslant s$ $\mathbb{J}: \mathbb{A} \ll \mathbb{E}\}$. Since $\mathbb{T} \in \mathcal{S} \mathcal{E} \mathcal{P}_{N}$ (because $\mathscr{X}$ is standard), $s(\mathbb{T}) \in \mathcal{S} \mathcal{E} \mathcal{P}_{N}$ as well. So, if $\mathbb{X} \ll \mathbb{T}$, then $s(\mathbb{X}) \leqslant s(\mathbb{T})$ and consequently the set $J=\left\{(i, \alpha) \in \Upsilon: \mathbb{E}_{\alpha}^{i}(\mathbb{X}) \neq \mathbb{O}\right\}$ is countable.

We infer from Lemma 20.9 (page 83) that:

- $\mathbb{T}$ is the direct sum of a minimal $N$-tuple and a semiminimal one,

- there is $\lambda \in \operatorname{rgm}\left(\mathfrak{p}_{N}\right)$ such that $\mathbb{T}=\int_{\mathfrak{p}_{N}}^{\boxplus} \mathbb{P} \mathrm{d} \lambda(\mathbb{P})$,

- for each $(i, \alpha) \in J$ there is a Borel function $u_{\alpha}^{i}: \mathfrak{p}_{N} \rightarrow I_{\aleph_{0}}$ such that $u_{\alpha}^{i}\left(\mathfrak{a}_{N}\right) \subset$ Card, $u_{\alpha}^{i}\left(\mathfrak{f}_{N}\right) \subset\left\{0, \aleph_{0}\right\}$ and

$$
\begin{array}{ll}
\mathbb{E}_{\alpha}^{i}(\mathbb{X})=\int_{\mathfrak{p}_{N}}^{\boxplus} u_{\alpha}^{i}(\mathbb{P}) \odot \mathbb{P} \mathrm{d} \lambda(\mathbb{P}) & \text { if }(i, \alpha) \neq(I I, 1), \\
\mathbb{E}_{s m}(\mathbb{X})=\int_{\mathfrak{p}_{N}}^{\boxplus} u_{\alpha}^{i}(\mathbb{P}) \odot \mathbb{P} \mathrm{d} \lambda(\mathbb{P}) & \text { if }(i, \alpha)=(I I, 1) .
\end{array}
$$

Further, it follows from Corollary 20.7 (page 81) that

$$
\Phi^{*}(\mu) \ll \lambda \ll \Phi^{*}(\mu)
$$

(cf. (22-5)). Since $\mathscr{X}$ is standard and $\Phi \in \operatorname{RGS}_{l o c}(\mathscr{X})$, we may assume that $\Phi$ is a Borel isomorphism of $\mathscr{X}$ onto a measurable domain. Put $g_{\alpha}^{i}=u_{\alpha}^{i} \circ \Phi$ for $(i, \alpha) \in J$ and note that $g_{\alpha}^{i} \in \mathscr{A}(\mathscr{X})$. Now (22-5), (22-6) and (22-7) combined with (DI4) (page 77) for every $(i, \alpha) \in J$ 
yield

$$
\begin{aligned}
& \mathbb{E}_{\alpha}^{i}(\mathbb{X})=\bigoplus_{x \in \mathscr{X}}^{\mathcal{N}} g_{\alpha}^{i}(x) \odot \Phi(x) \quad \text { if }(i, \alpha) \neq(I I, 1), \\
& \mathbb{E}_{s m}(\mathbb{X})=\bigoplus_{x \in \mathscr{X}}^{\mathcal{N}} g_{\alpha}^{i}(x) \odot \Phi(x) \quad \text { if }(i, \alpha)=(I I, 1)
\end{aligned}
$$

Let $(i, \alpha)$ and $\left(i^{\prime}, \alpha^{\prime}\right)$ be distinct elements of $J$. Suppose $s\left(g_{\alpha}^{i}\right) \cap s\left(g_{\alpha^{\prime}}^{i^{\prime}}\right) \notin$ $\mathcal{N}\left(s\left(g_{\alpha}^{i}\right)\right.$ 's are measurable since $g_{\alpha}^{i}$ 's are such). Then there is a nonnull measurable set $\mathscr{B}$ which is contained in $s\left(g_{\alpha}^{i}\right) \cap s\left(g_{\alpha^{\prime}}^{i^{\prime}}\right)$. Consequently, thanks to (CS3) and (22-8)-(22-9), $\aleph_{0} \odot \bigoplus_{x \in \mathscr{B}}^{\mathcal{N}} \Phi(x) \leqslant \aleph_{0} \odot \mathbb{E}_{\alpha}^{i}(\mathbb{X})$ as well as $\aleph_{0} \odot \bigoplus_{x \in \mathscr{B}}^{\mathcal{N}} \Phi(x) \leqslant \aleph_{0} \odot \mathbb{E}_{\alpha^{\prime}}^{i^{\prime}}(\mathbb{X})$ which is impossible since $\mathbb{E}_{\alpha}^{i}(\mathbb{X}) \perp_{u} \quad \mathbb{E}_{\alpha^{\prime}}^{i^{\prime}}(\mathbb{X})$ and $\boxplus_{x \in \mathscr{B}}^{\mathcal{N}} \Phi(x) \neq \mathbb{O}$. This proves that $s\left(g_{\alpha}^{i}\right) \cap$ $s\left(g_{\alpha^{\prime}}^{i^{\prime}}\right) \in \mathcal{N}$ for any different members $(i, \alpha)$ and $\left(i^{\prime}, \alpha^{\prime}\right)$ of $J$. It then follows from the countability of $J$ that there is $\mathscr{Z} \in \mathcal{N}$ such that the sets $\mathscr{S}_{\alpha}^{i}=s\left(g_{\alpha}^{i}\right) \backslash \mathscr{Z}((i, \alpha) \in J)$ are pairwise disjoint. Now we define $f: \mathscr{X} \rightarrow \mathbb{R}_{+} \cup$ Card by the rules: $f(x)=\alpha \cdot g_{\alpha}^{i}(x)$ for $x \in \mathscr{S}_{\alpha}^{i}$ with $(i, \alpha) \in J \backslash\{(I I, 1)\} ; f(x)=g_{1}^{I I}(x)$ for $x \in \mathscr{S}_{1}^{I I}$ provided $(I I, 1) \in J$; and $f(x)=0$ for $x \notin \bigcup_{(i, \alpha) \in J} \mathscr{S}_{\alpha}^{i}$. It follows from the construction that $f \in \mathscr{A}(\mathscr{X})$. Finally, Theorem 11.1 (page 26), (22-8)-(22-9), (CS2) and (CS3) (page 91) give $\mathbb{X}=\boxplus_{x \in \mathscr{X}}^{\mathcal{N}} f(x) \odot \Phi(x)$.

Theorem 22.5 asserts that $\mathcal{J}(\Phi)=\left\{\boxplus_{x \in \mathscr{X}}^{\mathcal{N}} f(x) \odot \Phi(x): f \in \mathscr{A}(\mathscr{X})\right\}$ is an ideal. We call a quadruple $(\mathscr{Y}, \mathfrak{N}, \mathcal{Z}, \Psi)$ or a pair $(\mathscr{Y}, \Psi)$ a covering

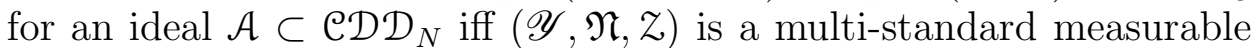
space with nullity, $\Psi \in \operatorname{RGS}_{l o c}(\mathscr{Y}), \Psi(\mathscr{Y}) \subset \mathfrak{p}_{N}$ and $\mathcal{J}(\Psi)=\mathcal{A}$ (with this terminology we are inspired by condition (ii) of Theorem 21.5, page 92 ). Whenever the ideal $\mathcal{A}$ is irrelevant, we shall speak shortly of a covering. A full covering is a covering for $\mathcal{C D} \mathcal{D}_{N}$.

As usual, whenever $\mathscr{D} \in \mathfrak{M}, j_{\mathscr{D}}$ stands for the characteristic function of $\mathscr{D}$.

22.6. Corollary. Let $f, g, h_{1}, h_{2}, \ldots \in \mathscr{A}(\mathscr{X}), \mathbb{X}=\boxplus_{x \in \mathscr{X}}^{\mathcal{N}} f(x) \odot \Phi(x)$ and $\mathbb{Y}=\boxplus_{x \in \mathscr{X}}^{\mathcal{N}} g(x) \odot \Phi(x)$.

(A) $\mathbb{X} \leqslant \mathbb{Y}$ iff $f \leqslant g$ a.e.

(B) $\mathbb{X} \perp_{u} \mathbb{Y}$ iff $f \cdot g=0$ a.e.

(C) $\mathbb{X} \ll \mathbb{Y}$ iff $s(f) \backslash s(g) \in \overline{\mathcal{N}}$.

(D) $\mathbb{X} \leqslant^{s} \mathbb{Y}$ iff $f=g \cdot j_{\mathscr{D}}$ a.e. for some $\mathscr{D} \in \mathfrak{M}$.

(E) $\boxplus_{x \in \mathscr{X}}^{\mathcal{N}}\left[\sum_{n=1}^{\infty} h_{n}(x)\right] \odot \Phi(x)=\bigoplus_{n=1}^{\infty}\left[\boxplus_{x \in \mathscr{X}}^{\mathcal{N}} h_{n}(x) \odot \Phi(x)\right]$.

Proof. Observe that point (D) is an immediate consequence of (21-5) (page 92) and Proposition 22.4; (B) follows from (A) and Theorem 22.5;

(E) is implied by (A), (CS3) (page 91) and (AO6) (page 29); while (C) follows from (CS3) and (B). It therefore suffice to prove (A). Implication ' $\Longleftarrow$ ' is a consequence of (CS3) and point (d) of Corollary 22.2. 
Finally, the converse implication follows from Proposition 22.4 and Theorem 22.5. Indeed, if $\mathbb{X} \leqslant \mathbb{Y}$, there is $\mathbb{A} \in \mathcal{C D D}_{N}$ such that $\mathbb{Y}=\mathbb{X} \oplus \mathbb{A}$. Then $\mathbb{A} \in \mathcal{J}(\Phi)$ and consequently there is $h \in \mathscr{A}(\mathscr{X})$ for which $\mathbb{A}=\boxplus_{x \in \mathscr{X}}^{\mathcal{N}} h(x) \odot \Phi(x)$. We now deduce from (CS3) that $\boxplus_{x \in \mathscr{X}}^{\mathcal{N}} g(x) \odot \Phi(x)=\boxplus_{x \in \mathscr{X}}^{\mathcal{N}}(f+h)(x) \odot \Phi(x)$ and hence, by Proposition $22.4, g=f+h$ a.e.

For need of the next result, we put $\mathscr{X}_{I_{n}}=\Phi^{-1}\left(\mathfrak{a}_{N}(n)\right), \mathscr{X}_{I I_{1}}=$ $\Phi^{-1}\left(\mathfrak{s}_{N}(1)\right)$ and $\mathscr{X}_{I_{\infty}}=\Phi^{-1}\left(\mathfrak{s}_{N}(\infty)\right)$. Observe that all just defined sets are pairwise disjoint, $\mathscr{X}_{I}=\bigcup_{n=1}^{n=\infty} \mathscr{X}_{I_{n}}$ and $\mathscr{X}_{I I}=\mathscr{X}_{I I_{1}} \cup \mathscr{X}_{I_{\infty} \infty}$, and they are measurable if so is $\Phi$ (and this is our assumption, for simplicity).

22.7. Corollary. Let $f \in \mathscr{A}(\mathscr{X})$ and $\mathbb{A}=\boxplus_{x \in \mathscr{X}}^{\mathcal{N}} f(x) \odot \Phi(x)$.

(a) $\mathbb{A} \in \mathcal{M} \mathcal{F}_{N}$ (respectively $\mathbb{A} \in \mathcal{H} \mathcal{H J M}_{N} ; \mathbb{A} \in \mathcal{S M}_{N}$ ) iff $f=j_{\mathscr{D}}$ a.e. for some measurable $\mathscr{D} \subset \mathscr{X}_{I}$ (respectively $f=\aleph_{0} \cdot j_{\mathscr{D}}$ a.e. for some measurable $\mathscr{D} \subset \mathscr{X}_{I I I}$; there is $\mathscr{Z} \in \mathcal{N}$ such that $f\left(\left(\mathscr{X}_{I} \cup \mathscr{X}_{I I I}\right) \backslash\right.$ $\mathscr{Z}) \subset\{0\}$ and $\left.f\left(\mathscr{X}_{I I} \backslash \mathscr{Z}\right) \subset \mathbb{R}_{+}\right)$. In particular, $\boxplus_{x \in \mathscr{X}}^{\mathcal{N}} \Phi(x)$ is the direct sum of a minimal $N$-tuple and a semiminimal one.

(b) $\mathbb{A} \in \mathcal{S E P}_{N}$ (respectively $\mathbb{A} \in \mathfrak{a}_{N} ; \mathbb{A} \in \mathfrak{f}_{N} ; \mathbb{A} \in \mathfrak{s}_{N} ; \mathbb{A} \in \mathfrak{F}_{N}$ ) iff there is $\mathscr{Z} \in \mathcal{N}$ such that $s(f) \backslash \mathscr{Z}$ is standard and $f(\mathscr{X} \backslash \mathscr{Z}) \subset I_{\aleph_{0}}$ (respectively $f=j_{\{x\}}$ a.e. for some $x \in \mathscr{X}_{I} \cap \mathscr{X}^{d} ; f=\aleph_{0} \cdot j_{\{x\}}$ a.e. for some $x \in \mathscr{X}_{I I I} \cap \mathscr{X}^{d} ; f=t \cdot j_{\{x\}}$ a.e. for some $x \in \mathscr{X}_{I I} \cap \mathscr{X}^{d}$ and $t \in \mathbb{R}_{+} \backslash\{0\} ; f=s \cdot j_{\{x\}}$ a.e. for some $x \in \mathscr{X}^{d}$ and $\left.s \in I_{\aleph_{0}} \backslash\{0\}\right)$.

(c) $\mathbb{A}$ is type $I ; I^{n} ; I I ; I I^{1} ; I I^{\infty} ; I I I$ iff, respectively, $s(f) \backslash \mathscr{X}_{I} ; s(f) \backslash$ $\mathscr{X}_{I_{n}} ; s(f) \backslash \mathscr{X}_{I I} ; s(f) \backslash \mathscr{X}_{I I_{1}} ; s(f) \backslash \mathscr{X}_{I I_{\infty}} ; s(f) \backslash \mathscr{X}_{I I I}$ is a member of $\overline{\mathcal{N}}$.

(d) $\mathbb{A}^{d}=\boxplus_{x \in \mathscr{X}^{d}} f(x) \odot \Phi(x)$ and $\mathbb{A}^{c}=\boxplus_{x \in \mathscr{X}^{c}}^{\mathcal{N}} f(x) \odot \Phi(x)$.

(e) Let $\mathscr{Z} \in \mathcal{N}$ be such that $\left.f\right|_{\mathscr{X} \backslash \mathscr{Z}}$ is Borel and $\mathscr{X} \backslash \mathscr{Z}$ is the union of a base $\mathcal{B}$ consisting of sets each of which is isomorphic either to $\left([0,1], \mathfrak{B}([0,1]), \mathcal{L}_{0}\right)$ or to a one-point nontrivial measurable space with nullity (there exists such $\mathscr{Z})$. Put $\mathscr{E}_{s m}=f^{-1}\left(\mathbb{R}_{+} \backslash\{0\}\right) \cap$ $\mathscr{X}_{I I} \backslash \mathscr{Z}$ and $\mathscr{E}_{\alpha}^{i}=f^{-1}(\{\alpha\}) \cap \mathscr{X}_{i} \backslash \mathscr{Z}$ for $(i, \alpha) \in \Upsilon_{*}$. Then $\mathcal{E}=\left\{\mathscr{E}_{\alpha}:(i, \alpha) \in \Upsilon_{*}\right\} \cup\left\{\mathscr{E}_{s m}\right\}$ is a base of $\mathscr{X}$, and $\mathbb{E}_{s m}(\mathbb{A})=$ $\boxplus_{x \in \mathscr{E}_{s m}}^{\mathcal{N}} f(x) \odot \Phi(x)$ and $\mathbb{E}_{\alpha}^{i}(\mathbb{A})=\boxplus_{x \in \mathscr{E}_{\alpha}^{i}}^{\mathcal{N}} \Phi(x)$ for $(i, \alpha) \in \Upsilon_{*}$ with $\alpha \neq 0$.

Proof. Points (a)-(d) are left as exercises. They are almost immediate consequences of Propositions 16.4 (page 57), 20.6 (page 80) and the fact that central decompositions of von Neumann algebras preserve the types. Note also that $\boxplus_{x \in \mathscr{X}^{d}}^{\mathcal{N}} f(x) \odot \Phi(x)=\boxplus_{x \in \mathscr{X}^{d}} f(x) \odot \Phi(x)$ since $\left.\mathcal{N}\right|_{\mathscr{C}^{d}}=\{\varnothing\}$. Here we shall focus on (e).

To prove (e), it suffices to show that $\mathcal{E}$ is a base of $\mathscr{X}$, since then the remainder will follow from (CS2), (CS3) (page 91), (a) and the uniqueness in Theorem 11.1 (page 26). It is clear that $\mathcal{E}$ consists of 
pairwise disjoint, measurable sets (because $f$ is measurable on $\mathscr{X} \backslash \mathscr{Z}$ ) and $\mathscr{X} \backslash \bigcup \mathcal{E}=\mathscr{Z}$. Now assume $A \subset \mathscr{X} \backslash \mathscr{Z}$ is such that $A \cap \mathscr{E} \in \mathfrak{M}$ (respectively $A \cap \mathscr{E} \in \mathcal{N}$ ) for any $\mathscr{E} \in \mathcal{E}$. Let $\mathcal{B}$ be as in (e). It follows from the proof of Lemma 21.4 (page 89) that $f(B) \cap$ Card $_{\infty}$ is countable for each $B \in \mathcal{B}$. Consequently, also the set $\mathcal{E}(B)=\{E \in \mathcal{E}: E \cap B \neq$ $\varnothing\}$ is countable and thus $A \cap B=\bigcup_{E \in \mathcal{E}(B)}[(A \cap E) \cap B]$ is a member of $\mathfrak{M}$ (respectively $\mathcal{N}$ ) for any $B \in \mathcal{B}$. Since $\mathcal{B}$ is a base, we obtain $A \in \mathfrak{M}(A \in \mathcal{N})$ and we are done.

22.8. Remark. For need of this remark, for every measurable set $\mathscr{D} \subset$ $\mathscr{X}$, let $\mathfrak{j}_{\mathscr{D}}$ denote an admissible function which is equal to 0 off $\mathscr{D}, 1$ on $\mathscr{D} \backslash \mathscr{X}_{I I I}$ and $\aleph_{0}$ on $\mathscr{D} \cap \mathscr{X}_{I I I}$.

Using point (E) of Corollary 22.6 as well as properties (CS2) and (CS3)-(B) (page 91), one may show that whenever $(\mathscr{X}, \mathfrak{M}, \mathcal{N}, \Phi)$ is a covering, the regular (continuous) direct sums of the form $\boxplus_{x \in \mathscr{X}}^{\mathcal{N}} f(x) \odot$ $\Phi(x)$ with $f \in \mathscr{A}(\mathscr{X})$ may axiomatically be defined by axioms (AX0)(AX3) stated below. Namely, it is now quite easy to prove that if $\Psi: \mathscr{A}(\mathscr{X}) \rightarrow \mathcal{C D D}_{N}$ is an assignment such that

$\left(\right.$ AX0) for every $\mathscr{D} \in \mathfrak{M}, \Psi\left(\mathfrak{j}_{\mathscr{D}}\right)=\boxplus_{x \in \mathscr{D}}^{\mathcal{N}} \Phi(x)$,

(AX1) whenever $\mathcal{B}$ is a base of $\mathscr{X}, \Psi(f)=\bigoplus_{B \in \mathcal{B}} \Psi\left(\mathfrak{j}_{B} \cdot f\right)$ for every $f \in \mathscr{A}(\mathscr{X})$,

(AX2) $\Psi(\alpha \cdot f)=\alpha \odot \Psi(f)$ for any $\alpha \in$ Card and $f \in \mathscr{A}(\mathscr{X})$, (AX3) $\Psi\left(\sum_{n=1}^{\infty} f_{n}\right)=\bigoplus_{n=1}^{\infty} \Psi\left(f_{n}\right)$ for all $f_{1}, f_{2}, \ldots \in \mathscr{A}(\mathscr{X})$,

then $\Psi(f)=\boxplus_{x \in \mathscr{X}}^{\mathcal{N}} f(x) \odot \Phi(x)$ for any $f \in \mathscr{A}(\mathscr{X})$ (to show this, use Corollary 22.7-(e) and the fact that a real-valued measurable function may be written as the series of rational-valued simple functions). However, at this moment we do not know whether $\Phi$ is uniquely determined (up to a.e. equality) by 'its' continuous direct sums appearing in (AX0). This (and even more) will be proved later, in Theorem 22.17 (page 106).

The next result follows from Corollary 22.7 and its proof is left for the reader.

22.9. Corollary. Let $(\mathscr{Y}, \Psi)$ be a covering for an ideal $\mathcal{A} \subset \mathcal{C D D}_{N}$ and let $\mathbb{B}=\mathbb{J}(\mathcal{A})$. Then $\iota^{d}(\mathscr{Y})=\operatorname{card}\left(\left\{\mathbb{X} \in \mathfrak{F}_{N}: \mathbb{X} \leqslant^{s} \mathbb{B}\right\}\right)$ and $\iota^{c}(\mathscr{Y})=\operatorname{dim}\left(\mathbb{B}^{c}\right)$.

Our next aim is to establish (in a sense) uniqueness (Theorem 22.10 and Corollary 22.11 below) and existence (Proposition 22.13) of coverings for arbitrary ideals in $\mathcal{C D} \mathcal{D}_{N}$.

22.10. Theorem. Let $\left(\mathscr{X}_{1}, \mathfrak{M}_{1}, \mathcal{N}_{1}, \Phi_{1}\right)$ and $\left(\mathscr{X}_{2}, \mathfrak{M}_{2}, \mathcal{N}_{2}, \Phi_{2}\right)$ be two coverings such that

$$
\bigoplus_{x \in \mathscr{X}_{1}}^{\mathcal{N}_{1}} \Phi_{1}(x)=\bigoplus_{x \in \mathscr{X}_{2}}^{\mathcal{N}_{2}} \Phi_{2}(x)
$$


Then there are sets $\mathscr{Z}_{j} \in \mathcal{N}_{j}(j=1,2)$ and a null-isomorphism $\tau: \mathscr{X}_{1} \backslash$ $\mathscr{Z}_{1} \rightarrow \mathscr{X}_{2} \backslash \mathscr{Z}_{2}$ such that $\Phi_{1}(x)=\Phi_{2}(\tau(x))$ for each $x \in \mathscr{X}_{1} \backslash \mathscr{Z}_{1}$.

Proof. Let $\mathcal{B}_{j}$ be a standard base of $\mathscr{X}_{j}$. For $B \in \mathcal{B}_{j}$ put $\mathbb{T}_{B}^{(j)}=$ $\bigoplus_{x \in B}^{\mathcal{N}_{j}} \Phi_{j}(x)$. It follows from (CS2) and (22-10) that

$$
\bigoplus_{B \in \mathcal{B}_{1}} \mathbb{T}_{B}^{(1)}=\bigoplus_{B \in \mathcal{B}_{2}} \mathbb{T}_{B}^{(2)}
$$

Let $I=\left\{\left(B_{1}, B_{2}\right) \in \mathcal{B}_{1} \times \mathcal{B}_{2} \mid \quad \mathbb{T}_{B_{1}, B_{2}}:=\mathbb{T}_{B_{1}}^{(1)} \wedge \mathbb{T}_{B_{2}}^{(2)} \neq \mathbb{O}\right\}$. We conclude from (22-11) that

$$
\begin{aligned}
& \mathbb{T}_{B}^{(1)}=\bigoplus\left\{\mathbb{T}_{B, B^{\prime}}:\left(B, B^{\prime}\right) \in I\right\} \quad\left(B \in \mathcal{B}_{1}\right), \\
& \mathbb{T}_{B}^{(2)}=\bigoplus\left\{\mathbb{T}_{B^{\prime}, B}:\left(B, B^{\prime}\right) \in I\right\} \quad\left(B \in \mathcal{B}_{2}\right) .
\end{aligned}
$$

It follows from Corollary 22.6 and (22-12)-(22-13) that sets $I_{2}\left(B^{\prime}\right)=$ $\left\{B_{2} \in \mathcal{B}_{2}:\left(B^{\prime}, B_{2}\right) \in I\right\}$ and $I_{1}\left(B^{\prime \prime}\right)=\left\{B_{1} \in \mathcal{B}_{1}:\left(B_{1}, B^{\prime \prime}\right) \in I\right\}$ are countable ( since $\mathbb{T}_{B}^{(j)} \in \mathcal{S} \mathcal{E} \mathcal{P}_{N}$ ) for any $B^{\prime} \in \mathcal{B}_{1}$ and $B^{\prime \prime} \in \mathcal{B}_{2}$ and thus there are families of pairwise disjoint sets $\left\{D_{B^{\prime}, B}^{1}\right\}_{B \in I_{2}\left(B^{\prime}\right)} \subset \mathfrak{M}_{1}$ and $\left\{D_{B, B^{\prime \prime}}^{2}\right\}_{B \in I_{1}\left(B^{\prime \prime}\right)} \subset \mathfrak{M}_{2}$ such that $B^{\prime}=\bigcup_{B \in I_{2}\left(B^{\prime}\right)} D_{B^{\prime}, B}^{1}, B^{\prime \prime}=$ $\bigcup_{B \in I_{1}\left(B^{\prime \prime}\right)} D_{B, B^{\prime \prime}}^{2}$ and

$$
\mathbb{T}_{B_{1}, B_{2}}=\bigoplus_{x \in D_{B_{1}, B_{2}}^{1}}^{\mathcal{N}_{1}} \Phi_{1}(x)=\bigoplus_{x \in D_{B_{1}, B_{2}}^{2}}^{\mathcal{N}_{2}} \Phi_{2}(x)
$$

for every $\left(B_{1}, B_{2}\right) \in I$ (cf. Corollary 22.6 or Theorem 21.5, page 92). We also infer from the countability of the sets $I_{1}\left(B_{2}\right)$ 's and $I_{2}\left(B_{1}\right)$ 's that

$$
\left\{D_{B_{1}, B_{2}}^{j}:\left(B_{1}, B_{2}\right) \in I\right\} \text { is a base of } \mathscr{X}_{j} .
$$

Fix $\left(B_{1}, B_{2}\right) \in I$. Since $D_{B_{1}, B_{2}}^{j}$ is standard and $\Phi_{j} \in \mathrm{RGS}_{l o c}$, there is a Borel set $G_{j} \subset D_{B_{1}, B_{2}}^{j}$ such that $D_{B_{1}, B_{2}}^{j} \backslash G_{j} \in \mathcal{N}_{j}, \Phi_{j}\left(G_{j}\right)$ is a measurable domain and $\left.\Phi_{j}\right|_{G_{j}}$ is a Borel isomorphism of $G_{j}$ onto $\Phi_{j}\left(G_{j}\right)$. Let $\mu_{j}$ be a standard measure on $\left.\mathfrak{M}_{j}\right|_{G_{j}}$ for which $\left.\mathcal{N}_{j}\right|_{G_{j}}=\mathcal{N}\left(\mu_{j}\right)$. Relation (22-14) yields that $\int_{G_{1}}^{\boxplus} \Phi_{1}(x) \mathrm{d} \mu_{1}(x)=\int_{G_{2}}^{\boxplus} \Phi_{2}(x) \mathrm{d} \mu_{2}(x)$. Hence Corollary 20.7 (page 81) implies that $\widehat{\mu}_{1} \ll \widehat{\mu}_{2} \ll \widehat{\mu}_{1}$ where $\widehat{\mu}_{j}(\mathcal{F})=$ $\mu_{j}\left(\Phi_{j}^{-1}(\mathcal{F}) \cap G_{j}\right)$ for $\mathcal{F} \in \mathfrak{B}\left(\mathfrak{p}_{N}\right)$. Consequently, $Z_{B_{1}, B_{2}}^{j}=D_{B_{1}, B_{2}}^{j} \backslash$ $\left[\Phi_{j}^{-1}\left(\Phi_{1}\left(G_{1}\right) \cap \Phi_{2}\left(G_{2}\right)\right) \cap G_{j}\right] \in \mathcal{N}_{j}$ and $\tau_{B_{1}, B_{2}}: D_{B_{1}, B_{2}}^{1} \backslash Z_{B_{1}, B_{2}}^{1} \ni x \mapsto$ $\left(\left.\Phi_{2}\right|_{G_{2}}\right)^{-1}\left(\Phi_{1}(x)\right) \in D_{B_{1}, B_{2}}^{2} \backslash Z_{B_{1}, B_{2}}^{2}$ is a well defined null-isomorphism such that

$$
\left\{\begin{array}{l}
\tau_{B_{1}, B_{2}}: D_{B_{1}, B_{2}}^{1} \backslash Z_{B_{1}, B_{2}}^{1} \rightarrow D_{B_{1}, B_{2}}^{2} \backslash Z_{B_{1}, B_{2}}^{2} \\
\Phi_{2} \circ \tau_{B_{1}, B_{2}}=\left.\Phi_{1}\right|_{D_{B_{1}, B_{2}}^{1} \backslash Z_{B_{1}, B_{2}}^{1}} .
\end{array}\right.
$$


Now it suffices to put $\mathscr{Z}_{j}=\left(\mathscr{X}_{j} \backslash \bigcup \mathcal{B}_{j}\right) \cup \bigcup_{\left(B_{1}, B_{2}\right) \in I} Z_{B_{1}, B_{2}}^{j}$ and define $\tau: \mathscr{X}_{1} \backslash \mathscr{Z}_{1} \rightarrow \mathscr{X}_{2} \backslash \mathscr{Z}_{2}$ as the union of $\left\{\tau_{B_{1}, B_{2}}\right\}_{\left(B_{1}, B_{2}\right) \in I}$. It follows from (22-15) and (22-16) that $\mathscr{Z}_{j} \in \mathcal{N}_{j}$ and $\tau$ is a null-isomorphism we searched for.

22.11. Corollary. Let $\mathcal{A} \subset \mathcal{C D} \mathcal{D}_{N}$ be an ideal and $\left(\mathscr{X}^{1}, \mathfrak{M}^{1}, \mathcal{N}^{1}, \Phi^{1}\right)$ and $\left(\mathscr{X}^{2}, \mathfrak{M}^{2}, \mathcal{N}^{2}, \Phi^{2}\right)$ be two coverings for $\mathcal{A}$. Then there are sets $\mathscr{Z}^{j} \in \mathcal{N}^{j}(j=1,2)$, a Borel function $u: \mathscr{X}^{1} \rightarrow \mathbb{R}_{+} \backslash\{0\}$ with $u\left(\mathscr{X}_{I}^{1} \cup\right.$ $\left.\mathscr{X}_{I I}^{1}\right) \subset\{1\}$, and a null-isomorphism $\tau: \mathscr{X}^{1} \backslash \mathscr{Z}^{1} \rightarrow \mathscr{X}^{2} \backslash \mathscr{Z}^{2}$ such that $\Phi^{2}(\tau(x))=u(x) \odot \Phi^{1}(x)$ for every $x \in \mathscr{X}^{1} \backslash \mathscr{Z}^{1}$.

Proof. Let $\mathbb{T}_{j}=\boxplus_{x \in \mathscr{X}^{j}}^{\mathcal{N}^{j}} \Phi^{j}(x)$. It follows from the assumptions and Theorem 22.5 that

$$
\mathbb{T}_{1} \ll \mathbb{T}_{2}
$$

and there is $f \in \mathscr{A}\left(\mathscr{X}^{1}\right)$ such that

$$
\mathbb{T}_{2}=\bigoplus_{x \in \mathscr{X}^{1}}^{\mathcal{N}^{1}} f(x) \odot \Phi^{1}(x) .
$$

Now Corollary 22.7 implies that $\mathbb{T}_{2}$ is the direct sum of a minimal $N$-tuple and a semiminimal one, and consequently there is $Z \in \mathcal{N}^{1}$ such that $A:=s(f) \backslash Z \in \mathfrak{M}^{1},\left.f\right|_{A}$ is Borel, $f\left(A \cap \mathscr{X}_{I}^{1}\right) \subset\{1\}$, $f\left(A \cap \mathscr{X}_{I I I}^{1}\right) \subset\left\{\aleph_{0}\right\}$ and $f\left(A \cap \mathscr{X}_{I I}^{1}\right) \subset \mathbb{R}_{+} \backslash\{0\}$. Further, Corollary 22.6 combined with (22-17) yields that $\mathscr{X}^{1} \backslash s(f) \in \overline{\mathcal{N}^{1}}$ and hence $\mathscr{X}^{1} \backslash A \in$ $\mathcal{N}^{1}$. Define $u: \mathscr{X}^{1} \rightarrow \mathbb{R}_{+} \backslash\{0\}$ by $u(x)=f(x)$ for $x \in A \backslash \mathscr{X}_{I I I}$ and $u(x)=1$ otherwise. Observe that $u$ is Borel and fits to $\Phi^{1}$, and $u(x) \odot \Phi^{1}(x)=f(x) \odot \Phi^{1}(x)$ for $x \in A$. So, (22-18) gives

$$
\bigoplus_{x \in \mathscr{X}^{2}}^{\mathcal{N}^{2}} \Phi^{2}(x)=\bigoplus_{x \in \mathscr{X}^{1}}^{\mathcal{N}^{1}} u(x) \odot \Phi^{1}(x) .
$$

Finally, since $u$ is real-valued, $u \odot \Phi^{1}: \mathscr{X}^{1} \rightarrow \mathfrak{p}_{N}$ and we deduce from Theorem 21.5 (page 92 ) that $\left(\mathscr{X}^{1}, u \odot \Phi^{1}\right)$ is a covering. So, the assertion follows from Theorem 22.10, thanks to (22-19).

To establish existence of coverings, we need the following

22.12. Lemma. Let $\mathscr{E} \subset \operatorname{rgm}\left(\mathfrak{p}_{N}\right)$ be such a family that

$$
\mu \perp_{s} \nu \text { if } \mu \neq \nu \text { and } \mu, \nu \in \mathscr{E} \text {. }
$$

Let $(\mathscr{X}, \mathfrak{M}, \mathcal{N})=\bigoplus_{\mu \in \mathscr{E}}\left(\mathfrak{p}_{N}, \mathfrak{B}\left(\mathfrak{p}_{N}\right), \mathcal{N}(\mu)\right)$ and $\Phi: \mathscr{X} \rightarrow \mathfrak{p}_{N}$ be the canonical projection. Then $(\mathscr{X}, \Phi)$ is a covering and

$$
\bigoplus_{x \in \mathscr{X}}^{\mathcal{N}} \Phi(x)=\bigoplus_{\mu \in \mathscr{E}} \int_{\mathfrak{p}_{N}}^{\boxplus} \mathbb{P} \mathrm{d} \mu(\mathbb{P}) \text {. }
$$

Proof. First of all, the usage of ' $\boxplus_{\mu \in \mathscr{E}}$ ' in the right-hand side expression of (22-21) is allowed by Lemma 20.10 (page 85), thanks to (22-20). Further, since regularity measures are concentrated on measurable domains 
which are Souslin-Borel sets, $(\mathscr{X}, \mathfrak{M}, \mathcal{N})$ is a multi-standard measurable space with nullity and $\left\{\mathfrak{p}_{N} \times\{\mu\}\right\}_{\mu \in \mathscr{E}}$ is a standard base of $\mathscr{X}$. Thus, it suffices to check that $\Phi \in \mathrm{RGS}_{l o c}(\mathscr{X})$ (then (22-21) will automatically be satisfied). It is clear that $\Phi$ is Borel.

Let $A \in \mathfrak{M}$ be standard. We will show that condition (ii) of Theorem 21.5 (page 92) is fulfilled. Since $A$ is standard, the set $\mathscr{E}^{\prime}=\{\mu \in$ $\mathscr{E}: \Phi(A) \notin \mathcal{N}(\mu)\}$ is countable. Observe that $Z_{0}=A \cap\left[\bigcup_{\mu \notin \mathscr{E}^{\prime}}\left(\mathfrak{p}_{N} \times\right.\right.$ $\{\mu\})]$ belongs to $\mathcal{N}$. Since $A \backslash Z_{0} \subset \mathfrak{p}_{N} \times \mathscr{E}^{\prime} \in \mathfrak{M}$, we may assume that

$$
A=\mathfrak{p}_{N} \times \mathscr{E}^{\prime}
$$

For $\mu \in \mathscr{E}^{\prime}$ let $\mathbb{T}_{\mu}=\int_{\mathfrak{p}_{N}}^{\boxplus} \mathbb{P} \mathrm{d} \mu(\mathbb{P})$. Put $\mathbb{T}=\boxplus_{\mu \in \mathscr{E}^{\prime}} \mathbb{T}_{\mu}$. It follows from point (C) of Lemma 20.9 (page 83) that $\mathbb{T}_{\mu}\left(\mu \in \mathscr{E}^{\prime}\right)$ is the direct sum of a minimal $N$-tuple and a semiminimal one, and thus so is $\mathbb{T}$. Moreover, since $\mathscr{E}^{\prime}$ is countable, $\mathbb{T} \in \mathcal{S} \mathcal{E} \mathcal{P}_{N}(\mathbb{T} \neq \mathbb{O}$ because standard sets are nonnull). Now point (A) of Lemma 20.9 asserts that there is a measure $\lambda \in \operatorname{rgm}\left(\mathfrak{p}_{N}\right)$ such that $\mathbb{T}=\int_{\mathfrak{p}_{N}}^{\boxplus} \mathbb{P} d \lambda(\mathbb{P})$. Since $\mathbb{T}_{\mu} \leqslant s \mathbb{T}$, we conclude from Corollary 20.7 (page 81 ) that

$$
\mu \ll \lambda \quad\left(\mu \in \mathscr{E}^{\prime}\right) .
$$

Further, it follows from (22-20) and the countability of $\mathscr{E}^{\prime}$ that there is a collection $\left\{S_{\mu}\right\}_{\mu \in \mathscr{E}^{\prime}}$ of pairwise disjoint measurable subsets of $\mathfrak{p}_{N}$ such that $\mu\left(\mathfrak{p}_{N} \backslash S_{\mu}\right)=0$ for every $\mu \in \mathscr{E}^{\prime}$. Finally, let $\mathcal{F} \subset \mathfrak{p}_{N}$ be a measurable domain such that $\lambda\left(\mathfrak{p}_{N} \backslash \mathcal{F}\right)=0$. Put

$$
D=\bigcup_{\mu \in \mathscr{E}^{\prime}}\left[\left(S_{\mu} \cap \mathcal{F}\right) \times\{\mu\}\right] .
$$

Observe that $D \subset A$ (by (22-22)), $A \backslash D \in \mathcal{N}\left(\mathfrak{p}_{N} \backslash\left(S_{\mu} \cap \mathcal{F}\right) \in \mathcal{N}(\mu)\right.$ by (22-23)), $\left.\Phi\right|_{D}$ is one-to-one (since the sets $S_{\mu}$ 's are pairwise disjoint) and $\Phi(D) \subset \mathcal{F}$. So, Remark 20.5 (page 80) finishes the proof.

22.13. Proposition. Let $\mathbb{T} \in \mathcal{C D D}_{N}$ be the direct sum of a minimal $N$-tuple and a semiminimal one. There is a covering $(\mathscr{X}, \mathfrak{M}, \mathcal{N}, \Phi)$ such that

$$
\mathbb{T}=\bigoplus_{x \in \mathscr{X}}^{\mathcal{N}} \Phi(x) .
$$

Proof. By Zorn's lemma, there is a maximal family $\mathscr{E} \subset \operatorname{rgm}\left(\mathfrak{p}_{N}\right)$ such that $(22-20)$ is satisfied and $\mathbb{T}_{\mu}:=\int_{\mathfrak{p}_{N}}^{\boxplus} \mathbb{P} \mu(\mathbb{P}) \leqslant^{s} \mathbb{T}$ for each $\mu \in \mathscr{E}$ (since $\mathbb{T}_{\mu} \neq \mathbb{O}$; cf. Lemma 9.1, page 22 ). It follows from Lemma 22.12 and its proof that $\boxplus_{\mu \in \mathscr{E}} \mathbb{T}_{\mu} \leqslant{ }^{s} \mathbb{T}$ and that it is enough to show that $\mathbb{X}:=\mathbb{T} \boxminus\left(\boxplus_{\mu \in \mathscr{E}} \mathbb{T}_{\mu}\right)$ is equal to $\mathbb{O}$. Suppose, for the contrary, that $\mathbb{X} \neq$ $\mathbb{O}$. Since $\mathbb{T} \leqslant \mathbb{J}$, we infer from Proposition 9.10 (page 24) that there is $\mathbb{Y} \in \mathcal{S} \mathcal{E} \mathcal{P}_{N}$ such that $\mathbb{Y} \leqslant^{s} \mathbb{X}$. Then $\mathbb{Y}$ is the direct sum of a minimal $N$-tuple and a semiminimal one (because $\mathbb{X} \leqslant s \mathbb{T}$ ). Now Lemma 20.9 (page 83) yields that there is $\nu \in \operatorname{rgm}\left(\mathfrak{p}_{N}\right)$ such that $\int_{\mathfrak{p}_{N}}^{\boxplus} \mathbb{P} \mathrm{d} \nu(\mathbb{P})=$ $\mathbb{Y}\left(\leqslant^{s} \mathbb{T}\right)$. Finally, since $\mathbb{Y} \perp_{u} \mathbb{T}_{\mu}$ for every $\mu \in \mathscr{E}$, Lemma 20.10 
(page 85) asserts that $\nu \perp_{s} \mu$ for any $\mu \in \mathscr{E}$, contradictory to the fact that $\mathscr{E}$ is maximal.

The next theorem is an immediate consequence of all previously established properties. This result may be formulated for arbitrary coverings. Our main interest however are full ones. To make the theorem most transparent, we repeat some of properties proved earlier.

22.14. Theorem (Prime Decomposition). (I) There exists a full covering. What is more, for every $\mathbb{T} \in \mathcal{S M}_{N}$ with $\aleph_{0} \odot \mathbb{T}=\mathbb{J}_{I I}$ there is a full covering $(\mathscr{X}, \mathfrak{M}, \mathcal{N}, \Phi)$ such that $\boxplus_{x \in \mathscr{X}}^{\mathcal{N}} \Phi(x)=$ $\mathbb{J}_{I} \boxplus \mathbb{T} \boxplus \mathbb{J}_{I I I}$.

(II) Let $\left(\mathscr{X}^{1}, \mathfrak{M}^{1}, \mathcal{N}^{1}, \Phi^{1}\right)$ and $\left(\mathscr{X}^{2}, \mathfrak{M}^{2}, \mathcal{N}^{2}, \Phi^{2}\right)$ be two full coverings. There are a Borel function $u: \mathscr{X}^{1} \rightarrow \mathbb{R}_{+} \backslash\{0\}$ such that $u\left(\mathscr{X}_{I}^{1} \cup \mathscr{X}^{I I I}\right)=\{1\}$ and an almost null-isomorphism $\tau: \mathscr{X}^{1} \rightarrow$ $\mathscr{X}^{2}$ such that $\Phi^{2} \circ \tau=u \odot \Phi^{1}$ a.e. In particular, for every $f \in \mathscr{A}\left(\mathscr{X}^{2}\right),(f \circ \tau) u \in \mathscr{A}\left(\mathscr{X}^{1}\right)$ and

$$
\bigoplus_{x \in \mathscr{X}^{2}}^{\mathfrak{N}^{2}} f(x) \odot \Phi^{2}(x)=\bigoplus_{x \in \mathscr{X}^{1}}^{\mathcal{N}^{1}}[(f \circ \tau) u](x) \odot \Phi^{1}(x) .
$$

(III) Let ( $\left.\mathscr{X}, \mathfrak{M}, \mathcal{N},\left\{\mathbb{P}_{x}\right\}_{x \in \mathscr{X}}\right)$ be a full covering.

(A) For each $\mathbb{A} \in \mathcal{C D D}_{N}$ there is $f \in \mathscr{A}(\mathscr{X})$ such that $\mathbb{A}=$ $\boxplus_{x \in \mathscr{X}}^{\mathcal{N}} f(x) \odot \mathbb{P}_{x}$.

(B) For every $f_{1}, f_{2}, f_{3}, \ldots \in \mathscr{A}(\mathscr{X}), \boxplus_{x \in \mathscr{X}}^{\mathcal{N}}\left[\sum_{n=1}^{\infty} f_{n}(x)\right] \odot \mathbb{P}_{x}=$ $\bigoplus_{n=1}^{\infty}\left[\boxplus_{x \in \mathscr{X}}^{\mathcal{N}} f_{n}(x) \odot \mathbb{P}_{x}\right]$

(C) Let $f, g \in \mathscr{A}(\mathscr{X})$. Put $\mathbb{X}=\boxplus_{x \in \mathscr{X}}^{\mathcal{N}} f(x) \odot \mathbb{P}_{x}$ and $\mathbb{Y}=$ $\boxplus_{x \in \mathscr{X}}^{\mathcal{N}} g(x) \odot \mathbb{P}_{x}$. Then:
(a) $\mathbb{X}=\mathbb{Y} \Longleftrightarrow f=g$ a.e.,
(b) $\mathbb{X} \leqslant \mathbb{Y} \Longleftrightarrow f \leqslant g$ a.e.,
(c) $\mathbb{X} \leqslant s \mathbb{Y} \Longleftrightarrow f=g \cdot j_{\mathscr{D}}$ a.e. for some $\mathscr{D} \in \mathfrak{M}$,
(d) $\mathbb{X} \ll \mathbb{Y} \Longleftrightarrow s(f) \backslash s(g) \in \overline{\mathcal{N}}$,
(e) $\mathbb{X} \perp_{u} \mathbb{Y} \Longleftrightarrow f \cdot g=0$ a.e. $\Longleftrightarrow s(f) \cap s(g) \in \overline{\mathcal{N}}$,
(f) $\alpha \odot \mathbb{X}=\boxplus_{x \in \mathscr{X}}^{\mathcal{N}}(\alpha \cdot f)(x) \odot \mathbb{P}_{x}$ for any $\alpha \in \mathrm{Card}$,
$(\mathrm{g}) \mathbb{X} \in \mathcal{S \mathcal { M }}_{N} \Longleftrightarrow s(f) \backslash \mathscr{X}_{I I} \in \overline{\mathcal{N}}$ and $f^{-1}\left(\operatorname{Card}_{\infty}\right) \in \overline{\mathcal{N}}$; $t \in \mathbb{R}_{+}$, if $\mathbb{X} \in \mathcal{S M}_{N}$, then $t \odot \mathbb{X}=\boxplus_{x \in \mathscr{X}}^{\mathcal{N}}[t \cdot f(x)] \odot \mathbb{P}_{x}$ for each

(h) $\mathbb{X} \in \mathcal{S} \mathcal{E} \mathcal{P}_{N}$ iff there is $\mathscr{Z} \in \mathcal{N}$ such that $s(f) \backslash \mathscr{Z}$ is standard and $f(\mathscr{X} \backslash \mathscr{Z}) \subset I_{\aleph_{0}}$.

We leave the proofs of point (g) and of a part of point (II) of Theorem 22.14 as exercises.

Theorem 22.14 says that after fixing $\mathbb{T} \in \mathcal{S} \mathcal{M}_{N}$ such that $\aleph_{0} \odot \mathbb{T}=$ $\mathbb{J}_{I I}$, there is a unique (up to almost null-isomorphism) full covering $(\mathscr{X}, \mathfrak{M}, \mathcal{N}, \Phi)$ such that $\boxplus_{x \in \mathscr{X}_{I I}}^{\mathcal{N}} \Phi(x)=\mathbb{T}$. Then for every $\mathbb{A} \in \mathcal{C} \mathcal{D D}_{N}$ there is a unique (up to almost everywhere equality) function $\mathfrak{m} \in$ 
$\mathscr{A}(\mathscr{X})$ such that

$$
\mathbb{A}=\bigoplus_{x \in \mathscr{X}}^{\mathcal{N}} \mathfrak{m}(x) \odot \Phi(x)
$$

The function $\mathfrak{m}$ is called multiplicity function of $\mathbb{A}$ (relative to $\mathbb{T}$ ) (compare with Chapter 4 of [9]) and the formula (22-24) is called prime decomposition of $\mathbb{A}$ (relative to $\mathbb{T}$ ). One may check that $\mathbb{A} \in \mathcal{C D} \mathcal{D}_{N}$ has multiplicity function (respectively prime decomposition) of a unique (i.e. independent of the choice of $\mathbb{T}$ ) form iff $\mathbb{E}_{s m}(\mathbb{A})=0$ (respectively $\left.\mathbb{A} \perp_{u} \mathbb{I}_{I I}\right)$.

Since $\mathfrak{a}_{N}(n)$ for finite $n$ consists of bounded $N$-tuples, Theorem 22.14 implies that every $N$-tuple $\mathbb{X}$ whose type $\mathrm{I}_{\infty}$, II and III parts vanish admits a decomposition in the form $\mathbb{X}=\bigoplus_{n=1}^{\infty} \mathbb{X}^{(n)}$ where each $\mathbb{X}^{(n)}$ is bounded. So, under the notation of Examples 16.2 (page 56), every such $\mathbb{X}$ belongs to $\mathcal{J}[\mathrm{cl} \Omega(\mathrm{bd})]$.

22.15. Remark. Theorem 22.14 implies that all measurable spaces with nullities being ingredients of full coverings are almost isomorphic. One may therefore ask of their (common) characteristic numbers $\iota^{d}$ and $\iota^{c}$. Using results of the next section and Corollary 22.9 one may show that both of them are equal to $2^{\aleph_{0}}$. Even more: whenever $(\mathscr{X}, \Phi)$ is a full covering, for $\mathscr{Y} \in\left\{\mathscr{X}, \mathscr{X}_{I}, \mathscr{X}_{I_{1}}, \mathscr{X}_{I_{2}}, \ldots, \mathscr{X}_{I_{\infty}}, \mathscr{X}_{I I}, \mathscr{X}_{I_{1}}, \mathscr{X}_{I_{\infty}}, \mathscr{X}_{I I I}\right\}$ one has $\iota^{d}(\mathscr{Y})=\iota^{c}(\mathscr{Y})=2^{\aleph_{0}}$.

22.16. Remark. There is a striking resemblance between Theorems 15.2 (page 46) and 22.14, and between the forms of $\Lambda(\Omega)$ (where $\Omega$ is an underlying model space) and of $\mathscr{A}(\mathscr{X}, \Psi)$ (where $(\mathscr{X}, \Psi)$ is a full covering). It is not a coincidence. When $(\mathscr{X}, \mathfrak{M}, \mathcal{N}, \Psi)$ is a full covering, $\mathcal{A}=L^{\infty}(\mathscr{X}, \mathfrak{M}, \mathcal{N})$ is a $\mathcal{W}^{*}$-algebra (since $\mathscr{X}$ is multi-standard-see the notes of the first paragraph of $\S 1.18$ in [29]). Now if $\Omega$ is the Gelfand spectrum of $\mathcal{A}$, there is a one-to-one correspondence between clopen subsets of $\Omega$ and members of $\mathfrak{M}$, which naturally correspond to $N$-tuples $\mathbb{X}$ such that $\mathbb{X} \leqslant^{s} \widetilde{\mathbb{T}}:=\mathbb{J}_{I} \boxplus \mathbb{T} \boxplus \mathbb{J}_{I I I}$ where $\mathbb{T}:=\boxplus_{x \in \mathscr{X}_{I I}}^{\mathcal{N}} \Psi(x)$. Since $\mathcal{Z}\left(\mathcal{W}^{\prime \prime}(\widetilde{T})\right)$ is isomorphic to $\mathcal{Z}\left(\mathcal{W}^{\prime \prime}(\boldsymbol{J})\right)$ (because $\widetilde{\mathbb{T}} \ll \mathbb{J} \ll \widetilde{\mathbb{T}}$; cf. (PR6), page 9), $\Omega$ is therefore homeomorphic to the Gelfand spectrum of $\mathcal{Z}\left(\mathcal{W}^{\prime \prime}(\boldsymbol{J})\right)$, that is, $\Omega$ is an underlying model space. Further, using results of Sections 15 and 22, one may show that there is a 'natural' correspondence, $f \mapsto \widehat{f}$, between $\Lambda(\Omega)$ and $\mathscr{A}(\mathscr{X})$ (induced by the isomorphism between $\mathcal{C}(\Omega)$ and $\mathcal{A})$ where in $\mathscr{A}(\mathscr{X})$ we identify functions which are equal almost everywhere. One may then check that the assignment

$$
\Lambda(\Omega) \ni f \mapsto \bigoplus_{x \in \mathscr{X}}^{\mathcal{N}} \widehat{f}(x) \odot \Psi(x)
$$

is inverse to $\Phi_{\mathbb{T}}$ introduced in Theorem 15.2. Thus $\mathscr{A}(\mathscr{X})$ may be considered as a 'concrete realization' of $\Lambda(\Omega)$. With such an approach, 
the multiplicity function $\mathfrak{m} \in \mathscr{A}(\mathscr{X})$ (relative to $\mathbb{T}$ ) of $\mathbb{X} \in \mathcal{S M}_{N}$ corresponds to $\frac{d \mathbb{X}}{d \mathbb{T}}$.

22.17. Theorem. Let $(\mathscr{X}, \mathfrak{M}, \mathcal{N})$ be a multi-standard measurable space with nullity.

(I) Let $\Phi: \mathscr{X} \rightarrow \mathfrak{p}_{N}$ be such that $(\mathscr{X}, \Phi)$ is a covering and let $\mu: \mathfrak{M} \rightarrow \mathcal{C D D}_{N}$ be given by

$$
\mu(\mathscr{A})=\bigoplus_{x \in \mathscr{A}}^{\mathcal{N}} \Phi(x) \quad(\mathscr{A} \in \mathfrak{M})
$$

Then:

$(\mathrm{M} 0) \mu(\mathscr{X})$ is the direct sum of a minimal $N$-tuple and a semiminimal one,

(M1) for every $\mathscr{A} \in \mathfrak{M}, \mu(\mathscr{A})=\mathbb{O} \Longleftrightarrow \mathscr{A} \in \mathcal{N}$,

(M2) whenever $\mathscr{A}$ and $\mathscr{B}$ are two measurable disjoint sets, $\mu(\mathscr{A} \cup$ $\mathscr{B})=\mu(\mathscr{A}) \boxplus \mu(\mathscr{B})$,

(M3) for every $\mathbb{A} \in \mathcal{C D D} \mathcal{D}_{N}$ such that $\mathbb{A} \leqslant^{s} \mu(\mathscr{X})$ there exists $\mathscr{A} \in \mathfrak{M}$ for which $\mu(\mathscr{A})=\mathbb{A}$.

(II) For every function $\mu: \mathfrak{M} \rightarrow \mathcal{C D D}_{N}$ satisfying conditions (M0)(M3) there exists a unique (up to almost everywhere equality) function $\Phi: \mathscr{X} \rightarrow \mathfrak{p}_{N}$ such that $(\mathscr{X}, \Phi)$ is a covering and (22-25) is fulfilled.

Proof. Point (I) is left for the reader. Here we focus only on (II).

Let $\mu$ be as in (II). Put $\mathbb{T}=\mu(\mathscr{X})$. Observe that:

(M4) for any $\mathscr{A}, \mathscr{B} \in \mathfrak{M}, \mu(\mathscr{A}) \leqslant^{s} \mu(\mathscr{B})$ iff $\mathscr{A} \backslash \mathscr{B} \in \mathcal{N}$,

(M5) $\{\mu(\mathscr{A}): \mathscr{A} \in \mathfrak{M}\}=\left\{\mathbb{A} \in \mathcal{C D D}_{N}: \mathbb{A} \leqslant^{s} \mathbb{T}\right\}$.

Indeed, (M5) easily follows from (M2) and (M3), because $\mathbb{T}=\mu(\mathscr{A}) \boxplus$ $\mu(\mathscr{X} \backslash \mathscr{A})$ for every measurable $\mathscr{A}$. To prove (M4), first of all note that

$$
\mu(\mathscr{A})=\mu(\mathscr{B}) \Longleftrightarrow(\mathscr{A} \backslash \mathscr{B}) \cup(\mathscr{B} \backslash \mathscr{A}) \in \mathcal{N},
$$

since, by $(\mathrm{M} 2), \mu(\mathscr{A})=\mu(\mathscr{A} \backslash \mathscr{B}) \boxplus \mu(\mathscr{A} \cap \mathscr{B}), \mu(\mathscr{B})=\mu(\mathscr{B} \backslash \mathscr{A}) \boxplus$ $\mu(\mathscr{A} \cap \mathscr{B})$ and (again thanks to $(\mathrm{M} 2)) \mu(\mathscr{A} \backslash \mathscr{B}) \perp_{u} \mu(\mathscr{B} \backslash \mathscr{A})$. These combined with (M1) give (22-26). Now if $\mathscr{A} \backslash \mathscr{B} \in \mathcal{N}$, we infer from $(22-26)$ that $\mu(\mathscr{A})=\mu(\mathscr{A} \cap \mathscr{B})$ and hence, by $(\mathrm{M} 2), \mu(\mathscr{B})=\mu(\mathscr{B} \backslash$ $\mathscr{A}) \boxplus \mu(\mathscr{A})$ which yields $\mu(\mathscr{A}) \leqslant^{s} \mu(\mathscr{B})$. Conversely, if the latter inequality is fulfilled, we conclude from (M5) that there is $\mathscr{C} \in \mathfrak{M}$ such that $\mu(\mathscr{C})=\mu(\mathscr{B}) \boxminus \mu(\mathscr{A})$. Since then (again by (M2)) $\mu(\mathscr{C} \cup$ $\mathscr{A})=\mu(\mathscr{C}) \boxplus \mu(\mathscr{A} \backslash \mathscr{C})=\mu(\mathscr{A}) \boxplus \mu(\mathscr{C} \backslash \mathscr{A}), \mu(\mathscr{C}) \perp_{u} \mu(\mathscr{A})$ and $\mu(\mathscr{A} \backslash \mathscr{C}) \perp_{u} \mu(\mathscr{C} \backslash \mathscr{A})$, we get $\mu(\mathscr{A})=\mu(\mathscr{A} \backslash \mathscr{C})$ and consequently $\mu(\mathscr{A} \cup \mathscr{C})=\mu(\mathscr{C}) \boxplus \mu(\mathscr{A})=\mu(\mathscr{B})$. So, (22-26) yields the assertion of (M4).

Further, it follows from (M0) and Proposition 22.13 that there is a covering $\left(\mathscr{X}^{\prime}, \mathfrak{M}^{\prime}, \mathcal{N}^{\prime}, \Psi\right)$ such that $\mathbb{T}=\boxplus_{x \in \mathscr{X}^{\prime}}^{\mathcal{N}^{\prime}} \Psi(x)$. Put $\mu^{\prime}: \mathfrak{M}^{\prime} \ni$ $\mathscr{A} \mapsto \boxplus_{x \in \mathscr{A}}^{\mathcal{N}^{\prime}} \Psi(x) \in \mathcal{C D} \mathcal{D}_{N}$. It may be infered from Proposition 22.4 
(page 95), Theorem 22.5 (page 96) and Corollary 22.6 (page 98) that conditions (M4) and (M5) as well as (22-26) are satisfied when $\mu, \mathfrak{M}$ and $\mathcal{N}$ are replaced by (respectively) $\mu^{\prime}, \mathfrak{M}^{\prime}$ and $\mathcal{N}^{\prime}$. Let $\mathcal{M}$ and $\mathcal{M}^{\prime}$ denote the quotient (abstract) Boolean $\sigma$-algebras $\mathfrak{M} / \mathcal{N}$ and $\mathfrak{M}^{\prime} / \mathcal{N}^{\prime}$ (respectively). We shall denote the equivalence class in $\mathcal{M}$ (in $\mathcal{M}^{\prime}$ ) of $\mathscr{A} \in \mathfrak{M}\left(\right.$ of $\left.\mathscr{A} \in \mathfrak{M}^{\prime}\right)$ by $[\mathscr{A}]_{\mathcal{N}}\left(\right.$ by $[\mathscr{A}]_{\mathcal{N}^{\prime}}$ ). (M4), (M5) and (22-26) for both $\mu$ and $\mu^{\prime}$ imply that the rule

$$
\tau\left([\mathscr{A}]_{\mathcal{N}}\right)=[\mathscr{B}]_{\mathcal{N}^{\prime}} \Longleftrightarrow \mu(\mathscr{A})=\mu^{\prime}(\mathscr{B})
$$

well defines an order isomorphism $\tau: \mathcal{M} \rightarrow \mathcal{M}^{\prime}$. One deduces from this that whenever $\tau\left([\mathscr{A}]_{\mathcal{N}}\right)=[\mathscr{B}]_{\mathcal{N}^{\prime}}$,

$$
\mathscr{A} \text { is standard } \Longleftrightarrow \mathscr{B} \text { is standard. }
$$

Since $\tau$ is an order isomorphism, it is an isomorphism of Boolean $\sigma$ algebras as well. Now an application of [27, Corollary 14.4.12] separately for every member of a standard base of $\mathscr{X}$ yields that there are sets $\mathscr{Z} \in \mathcal{N}$ and $\mathscr{Z}^{\prime} \in \mathcal{N}^{\prime}$, and a null-isomorphism $\varphi: \mathscr{X} \backslash \mathscr{Z} \rightarrow \mathscr{X}^{\prime} \backslash \mathscr{Z}^{\prime}$ such that $\tau\left([\mathscr{A}]_{\mathcal{N}}\right)=[\varphi(\mathscr{A} \backslash \mathscr{Z})]_{\mathcal{N}^{\prime}}$ for every $\mathscr{A} \in \mathfrak{M}$. In particular, $\mu(\mathscr{A})=\mu^{\prime}(\varphi(\mathscr{A} \backslash \mathscr{Z}))$ or, equivalently,

$$
\mu(\mathscr{A})=\bigoplus_{y \in \varphi(\mathscr{A} \backslash \mathscr{Z})}^{\mathcal{N}^{\prime}} \Psi(y)=\bigoplus_{x \in \mathscr{A} \backslash \mathscr{Z}}^{\mathcal{N}}(\Psi \circ \varphi)(x)
$$

for any $\mathscr{A} \in \mathfrak{M}$. So, to obtain (22-25) it suffices to define $\Phi: \mathscr{X} \rightarrow \mathfrak{p}_{N}$ as an arbitrary extension of $\Psi \circ \varphi$.

Now assume that $\Phi^{\prime}: \mathscr{X} \rightarrow \mathfrak{p}_{N}$ is another function such that $\left(\mathscr{X}, \Phi^{\prime}\right)$ is a covering and $\mu(\mathscr{A})=\bigoplus_{x \in \mathscr{A}}^{\mathcal{N}} \Phi^{\prime}(x)$ for every $\mathscr{A} \in \mathfrak{M}$. Then $\boxplus_{x \in \mathscr{X}}^{\mathcal{N}} \Phi(x)=\boxplus_{x \in \mathscr{X}}^{\mathcal{N}} \Phi^{\prime}(x)$ and consequently-by Theorem 22.10there is an almost null-isomorphism $\kappa: \mathscr{X} \rightarrow \mathscr{X}$ such that $\Phi^{\prime}=\Phi \circ \kappa$ almost everywhere. It suffices to check that $\kappa(x)=x$ for almost all $x \in \mathscr{X}$. Take $\mathscr{Z} \in \mathcal{N}$ such that $\left.\kappa\right|_{\mathscr{X} \backslash \mathscr{Z}}$ is a null isomorphism of $\mathscr{X} \backslash \mathscr{Z}$ onto its (measurable) range. For simplicity, for every $\mathscr{A} \in \mathfrak{M}$ put $\mathscr{A}_{*}=\mathscr{A} \backslash \mathscr{Z}$. Notice that then

$$
\bigoplus_{x \in \mathscr{A}_{*}}^{\mathcal{N}} \Phi(x)=\bigoplus_{x \in \kappa\left(\mathscr{A}_{*}\right)}^{\mathcal{N}} \Phi(x)
$$

This implies (cf. Proposition 22.4, page 95) that $\left(\mathscr{A}_{*} \backslash \kappa\left(\mathscr{A}_{*}\right)\right) \cup\left(\kappa\left(\mathscr{A}_{*}\right) \backslash\right.$ $\left.\mathscr{A}_{*}\right) \in \mathcal{N}$. Equivalently, $\left[\mathscr{A}_{*}\right]_{\mathcal{N}}=\left[\kappa\left(\mathscr{A}_{*}\right)\right]_{\mathcal{N}}$ for every $\mathscr{A} \in \mathfrak{M}$. Since $\mathscr{X}$ is multi-standard, it follows from the uniqueness in [27, Theorem 14.4.10] that $\kappa(x)=x$ almost everywhere and we are done.

\section{Classifichtion of ideals UP to isomorphism}

This section is the only part of the treatise in which we will compare ideals of tuples of different lengths (that is, ideals in $\mathcal{C D} \mathcal{D}_{N}$ as well as in $\mathcal{C D D}_{N^{\prime}}$ with $N^{\prime} \neq N$ ).

We begin with 
23.1. Example. It is known that every properly infinite or type I von Neumann algebra acting in a separable Hilbert space is singly generated ([37], [28], [11]). There are also known examples of singly generated type $\mathrm{II}_{1}$ factors $([11])$. Also tensor products of two singly generated von Neumann algebras acting in separable Hilbert spaces are singly generated ([28, Corollary 2.1]). Further, according to [29, Theorem 2.6.6], the $\mathcal{W}^{*}$-tensor product of a type $\mathrm{I}_{n}, \mathrm{II}_{1}, \mathrm{II}_{\infty}$ or III $\mathcal{W}^{*}$-algebra and $L^{\infty}([0,1])$ is of the same type. Also, for a factor $\mathcal{M}$,

$$
\mathcal{Z}\left(\mathcal{M} \bar{\otimes} L^{\infty}([0,1])\right) \cong L^{\infty}([0,1]),
$$

by [29, Proposition 2.6.7] or [34, Corollary IV.5.11]. Finally, if $T$ is a bounded operator and $\boldsymbol{T}=(T, \ldots, T) \in \mathrm{CDD}_{N}$, then $\mathcal{W}(T)=\mathcal{W}(\boldsymbol{T})$. All these notices yield that the ideals $\mathcal{J}_{I_{n}}^{c}, \mathcal{J}_{I I_{1}}^{c}, \mathcal{J}_{I I_{\infty}}^{c}$ and $\mathcal{J}_{I I I}^{c}$ are nonntrivial. (Indeed, take a singly generated factor $\mathcal{M}$ acting in a separable Hilbert space of a fixed type $i$ and let $T$ be a generator of $\mathcal{M} \bar{\otimes} L^{\infty}([0,1])$. Then $\boldsymbol{T}=(T, \ldots, T) \in \mathcal{J}_{i}^{c}$, by $(23-1)$.)

23.2. Corollary. Let $\Omega$ denote the underlying model space for $\mathcal{C D} \mathcal{D}_{N}$. Each of the spaces $\Omega, \Omega_{I}, \Omega_{I_{n}}(n=1,2, \ldots, \infty), \Omega_{I I}, \Omega_{I I_{1}}, \Omega_{I_{\infty}}$ and $\Omega_{\text {III }}$ is homeomorphic to the topological disjoint union of $\beta D\left(2^{\aleph_{0}}\right)$ and $\beta\left[D\left(2^{\aleph_{0}}\right) \times \mathfrak{X}\right]$ where $D\left(2^{\aleph_{0}}\right)$ is the discrete space of power $2^{\aleph_{0}}$ and $\mathfrak{X}$ is the Gelfand spectrum of $L^{\infty}([0,1])$.

Proof. By Theorem 17.12 (page 63), it suffice to show that $\kappa_{c}(E)=2^{\aleph_{0}}$ where $E$ denotes any of the sets under the question. Equivalently (cf. Proposition 15.5, page 50), this is to say that $\operatorname{dim}(\mathbb{J}(\mathcal{A}))=2^{\aleph_{0}}$ where $\mathcal{A}$ is one of $\mathcal{J}_{I_{n}}^{c}, \mathcal{J}_{I I_{1}}^{c}, \mathcal{J}_{I I_{\infty}}^{c}, \mathcal{J}_{I I I}^{c}$. To simplify the argument, let $(i, k, \mathfrak{Z})$ be one of $\left(I, n, \mathfrak{a}_{N}(n)\right)$ (where $\left.n \in\{1,2, \ldots, \infty\}\right),\left(I I, 1, \mathfrak{s}_{N}(1)\right)$, $\left(I I, \infty, \mathfrak{s}_{N}(\infty)\right),\left(I I I, \infty, \mathfrak{f}_{N}\right)$ and let $\mathcal{A}=\mathcal{J}_{i_{k}}^{c}$. By Example 23.1 we know $\mathcal{A}$ is nontrivial. Hence (e.g. by Proposition 9.10, page 24) there is $\mathbb{T}_{0} \in \mathcal{A} \cap \mathcal{S} \mathcal{E} \mathcal{P}_{N}$ which is either minimal or semiminimal. Now according to Lemma 20.9 (page 83), there is $\mu_{0} \in \operatorname{rgm}\left(\mathfrak{p}_{N}\right)$ such that $\mathbb{T}_{0}=\int_{\mathfrak{p}_{N}}^{\boxplus} \mathbb{P} \mathrm{d} \mu_{0}(\mathbb{P})$. There is a measurable domain $\mathcal{F}$ on which $\mu$ concentrates. Since $\mu$ is standard, we may assume that $\mathcal{F}$ is a standard Borel space, and that $\mathcal{F} \subset \mathfrak{Z}$, by Corollary 22.7-(c) (page 99). We infer from the fact that $\mathbb{A}^{c}=\mathbb{A}$ that $\mu_{0}$ is nonatomic and consequently that $\mathcal{F}$ is uncountable. So, $\mathcal{F}$ is Borel isomorphic to $[0,1]$ which implies that there is a family $\left\{\lambda_{t}\right\}_{t \in \mathbb{R}}$ of probabilistic nonatomic Borel measures on $\mathcal{F}$ which are mutually singular. Since every measure on $\mathcal{F}$ is a regularity measure, Lemma 20.10 (page 85) shows that $\mathbb{X}_{s}:=\int_{\mathcal{F}}^{\boxplus} \mathbb{P} \mathrm{d} \lambda_{s}(\mathbb{P}) \perp_{u} \int_{\mathcal{F}}^{\boxplus} \mathbb{P} \mathrm{d} \lambda_{t}(\mathbb{P})=\mathbb{X}_{t}$ for any distinct real numbers $s$ and $t$. Finally, again thanks to Corollary $22.7, \mathbb{X}_{s} \in \mathcal{A}$ (because $\mathcal{F} \subset \mathfrak{Z}$ and $\lambda_{s}$ is nonatomic) and $\mathbb{X}_{s}$ is minimal or semiminimal for every $s \in \mathbb{R}$. Consequently, $\mathbb{X}:=\boxplus_{s \in \mathbb{R}} \mathbb{X}_{s}$ is a minimal or semiminimal member of $\mathcal{A}$ as well. This gives $\mathbb{X} \leqslant \mathbb{J}(\mathcal{A})$ and therefore $\operatorname{dim}(\mathbb{J}(\mathcal{A})) \geqslant \operatorname{dim}(\mathbb{X})=2^{\aleph_{0}}$ (since $\mathbb{X}_{s} \in \mathcal{S} \mathcal{E} \mathcal{P}_{N}$ for each $s \in \mathbb{R}$ ). 
As an important consequence of Corollary 23.2 we obtain that the underlying model space for $\mathcal{C D} \mathcal{D}_{N}$ and its 'characteristic' subsets are independent of $N$. This will be crucial in our investigations. Hence, we may shortly speak of an underlying model space.

Everywhere below $\mathcal{A}$ and $\mathcal{B}$ denote arbitrary ideals in $\mathcal{C D} \mathcal{D}_{N}$ and $\mathcal{C D D}_{N^{\prime}}$ (respectively).

23.3. Definition. A function $\Phi: \mathcal{A} \rightarrow \mathcal{B}$ is an isomorphism iff $\Phi$ is a bijection and $\Phi\left(\bigoplus_{s \in S} \mathbb{A}_{s}\right)=\bigoplus_{s \in S} \Phi\left(\mathbb{A}_{s}\right)$ for every collection $\left\{\mathbb{A}_{s}\right\}_{s \in S} \subset$ $\mathcal{A}$ (where, of course, $S$ is a set). An isomorphism $\Phi: \mathcal{A} \rightarrow \mathcal{B}$ is

- an s-isomorphism iff $\operatorname{dim} \Phi(\mathbb{A})=\operatorname{dim} \mathbb{A}$ for every $\mathbb{A} \in \mathcal{A}$,

- a $t$-isomorphism iff for each $\mathbb{A} \in \mathcal{A}$ the following condition is fulfilled: $\Phi(\mathbb{A})$ is of type $i^{k}$ iff so is $\mathbb{A}$ where $i^{k}$ is one of $I^{n}$ $(n=1,2, \ldots, \infty), I I^{1}, I I^{\infty}, I I I$.

Two ideals are isomorphic, s-isomorphic or t-isomorphic if there exists an isomorphism of suitable kind between them.

Let 'i' be the empty, 's' or 't' prefix. We write $\mathcal{A} \cong{ }^{i} \mathcal{B}$ iff $\mathcal{A}$ and $\mathcal{B}$ are i-isomorphic. Additionally, we write $\mathcal{A} \preccurlyeq^{i} \mathcal{B}$ if $\mathcal{A} \cong{ }^{i} \mathcal{B}^{\prime}$ for some ideal $\mathcal{B}^{\prime} \subset \mathcal{B}$.

As it is easily seen, every t-isomorphism is a d-isomorphism. Therefore:

$$
\begin{aligned}
& \mathcal{A} \cong^{t} \mathcal{B} \Longrightarrow \mathcal{A} \cong^{s} \mathcal{B} \Longrightarrow \mathcal{A} \cong \mathcal{B}, \\
& \mathcal{A} \preccurlyeq^{t} \mathcal{B} \Longrightarrow \mathcal{A} \preccurlyeq^{s} \mathcal{B} \Longrightarrow \mathcal{A} \preccurlyeq \mathcal{B} .
\end{aligned}
$$

It is also clear that ' $\preccurlyeq^{i}$ ' is transitive, while ' $\cong$ ' ' is an equivalence.

The main tool of this section is the following

23.4. Theorem. If $\Phi: \mathcal{A} \rightarrow \mathcal{B}$ is a bijection such that

$$
\Phi(\mathbb{X} \oplus \mathbb{Y})=\Phi(\mathbb{X}) \oplus \Phi(\mathbb{Y})
$$

for any $\mathbb{X}, \mathbb{Y} \in \mathcal{A}$, then $\Phi$ is an isomorphism and $\Phi$ preserves all notions, features and operations appearing in (ST1)-(ST17) (pp. 34-35).

The above result is a generalization of Proposition 13.1 (page 36) and its proof goes similarly (see Section 13). In particular, for every isomorphism $\Phi: \mathcal{A} \rightarrow \mathcal{B}$ and each $\mathbb{A} \in \mathcal{A}$ one has: $\operatorname{dim} \Phi(\mathbb{A})$ is uncountable iff so is $\operatorname{dim}(\mathbb{A})$ and if this is the case, they are equal. So, $\Phi$ is an s-isomorphism if $\Phi$ preserves 'dim' for members of $\mathcal{S} \mathcal{E} \mathcal{P}$ (the prefix 's' is after 'separable'). One may also check that $\Phi$ preserves atoms, fractals, semiprimes (using their definitions and the note on page 60 after Definition 17.4), factor tuples (by Proposition 17.2, page 59) and types I, II and III. Consequently, $\Phi\left(\mathcal{A}^{d}\right)=\mathcal{B}^{d}$ and $\Phi\left(\mathcal{A}^{c}\right)=\mathcal{B}^{c}$.

We shall now define characteristics of ideals which turn out to be sufficient for answering the questions whether $\mathcal{A} \cong i \mathcal{B}$ or $\mathcal{A} \preccurlyeq^{i} \mathcal{B}$. 
23.5. Definition. For any $D \in\left\{I, I_{1}, I_{2}, \ldots, I_{\infty}, I I, I I_{1}, I I_{\infty}, I I I\right\}$ let

$$
\begin{gathered}
\chi_{D}^{d}(\mathcal{A})=\operatorname{card}\left(\left\{\mathbb{X}: \mathbb{X} \in \mathfrak{F}_{N} \cap \mathcal{J}_{D}, \mathbb{X} \leqslant^{s} \mathbb{J}(\mathcal{A})\right\}\right), \\
\chi_{D}^{c}(\mathcal{A})=\operatorname{dim}\left(\mathbb{J}\left(\mathcal{A}^{c} \cap \mathcal{J}_{D}\right)\right) \text { and } \chi_{D}(\mathcal{A})=\left(\chi_{D}^{d}(\mathcal{A}), \chi_{D}^{c}(\mathcal{A})\right) . \text { Finally, let } \\
\chi(\mathcal{A})=\left(\chi_{I}(\mathcal{A}) ; \chi_{I I}(\mathcal{A}) ; \chi_{I I I}(\mathcal{A})\right) \\
\chi_{s}(\mathcal{A})=\left(\chi_{I_{1}}^{d}(\mathcal{A}), \chi_{I_{2}}^{d}(\mathcal{A}), \ldots, \chi_{I_{\infty}}^{d}(\mathcal{A})\right), \\
\chi_{t}(\mathcal{A})=\left(\chi_{I_{1}}(\mathcal{A}) ; \chi_{I_{2}}(\mathcal{A}) ; \ldots, \chi_{I_{\infty}}(\mathcal{A}) ; \chi_{I I_{1}}(\mathcal{A}) ; \chi_{I I_{\infty}}(\mathcal{A})\right) .
\end{gathered}
$$

When comparing sequences (finite or infinite) of the same length whose entries are cardinals, ' $\leqslant$ ' will denote the coordinatewise order.

Let $\Omega$ be an underlying model space and let $\Psi_{N}=\Phi_{\mathbb{T}}$ : $\mathcal{C D} \mathcal{D}_{N} \rightarrow$ $\Lambda(\Omega)$ be as in Theorem 15.2 (page 46). For $E=\operatorname{supp}_{\Omega} \mathcal{A}$ we have (under the notation of Definition 23.5): $\chi_{D}^{d}(\mathcal{A})=\kappa_{d}\left(E \cap \Omega_{D}\right.$ ) and $\chi_{D}^{c}(\mathcal{A})=\kappa_{c}\left(E \cap \Omega_{D}\right)$ (cf. Proposition 15.5, page 50). So, according to Theorem 17.12 (page 63; below ' $\cong$ ' means 'homeomorphic'),

$$
\Omega_{D} \cap \operatorname{supp}_{\Omega} \mathcal{A} \cong \Omega_{D} \cap \operatorname{supp}_{\Omega} \mathcal{B} \Longleftrightarrow \chi_{D}(\mathcal{A})=\chi_{D}(\mathcal{B}) .
$$

As an application of Theorem 23.4, Theorem 15.2 (page 46), Corollary 23.2 and (23-3) we obtain

23.6. Theorem. Let $N$ and $N^{\prime}$ be arbitrary positive integers, $\mathcal{A} \subset$ $\mathrm{eDD}_{N}$ and $\mathcal{B} \subset \mathcal{C D D}_{N^{\prime}}$ be ideals.

(I) $\mathcal{C D} \mathcal{D}_{N} \cong t \mathcal{C D D}_{N^{\prime}}$. What is more, each entry of $\chi\left(\mathcal{C D D}_{N}\right)$, $\chi_{s}\left(\mathcal{C D D}_{N}\right)$ or $\chi_{t}\left(\mathcal{C D D}_{N}\right)$ is equal to $2^{\aleph_{0}}$.

(II) $\mathcal{A} \cong \mathcal{B} \Longleftrightarrow \chi(\mathcal{A})=\chi(\mathcal{B}) ; \mathcal{A} \preccurlyeq \mathcal{B} \Longleftrightarrow \chi(\mathcal{A}) \leqslant \chi(\mathcal{B})$.

(III) $\mathcal{A} \cong s \mathcal{B} \Longleftrightarrow \chi(\mathcal{A})=\chi(\mathcal{B})$ and $\chi_{s}(\mathcal{A})=\chi_{s}(\mathcal{B}) ; \mathcal{A} \preccurlyeq^{s} \mathcal{B} \Longleftrightarrow$ $\chi(\mathcal{A}) \leqslant \chi(\mathcal{B})$ and $\chi_{s}(\mathcal{A}) \leqslant \chi_{s}(\mathcal{B})$.

(IV) $\mathcal{A} \cong{ }^{t} \mathcal{B} \Longleftrightarrow \chi(\mathcal{A})=\chi(\mathcal{B})$ and $\chi_{t}(\mathcal{A})=\chi_{t}(\mathcal{B}) ; \mathcal{A} \preccurlyeq^{t} \mathcal{B} \Longleftrightarrow$ $\chi(\mathcal{A}) \leqslant \chi(\mathcal{B})$ and $\chi_{t}(\mathcal{A}) \leqslant \chi_{t}(\mathcal{B})$.

(V) Up to isomorphism (respectively t-isomorphism), there is only $\gamma$ $\left(2^{\aleph_{0}}\right)$ different ideals where $\gamma=\operatorname{card}\left(\left\{\alpha \in\right.\right.$ Card: $\left.\left.\alpha \leqslant 2^{\aleph_{0}}\right\}\right)$.

Proof. The second claim of (I) follows from Corollary 23.2 and Proposition 17.8 (page 62). Since (V) and the remainder of (I) follow from (IV), it is sufficient to prove points (II)-(IV). Since their proofs are based on the same idea, we focus on (IV) and skip proofs for (II) and (III).

Since representatives of members of $\mathcal{J}^{c}$ act in infinite-dimensional Hilbert spaces, Theorem 23.4 yields that if $\Phi: \mathcal{A} \rightarrow \mathcal{A}^{\prime} \subset \mathcal{B}$ is a tisomorphism, then necessarily $\chi(\mathcal{A})=\chi\left(\mathcal{A}^{\prime}\right) \leqslant \chi(\mathcal{B})$ and $\chi_{t}(\mathcal{A})=$ $\chi_{t}\left(\mathcal{A}^{\prime}\right) \leqslant \chi_{t}(\mathcal{B})$. Conversely, if $\chi(\mathcal{A}) \leqslant \chi(\mathcal{B})$ and $\chi_{t}(\mathcal{A}) \leqslant \chi_{t}(\mathcal{B})$ (respectively $\chi(\mathcal{A})=\chi(\mathcal{B})$ and $\chi_{t}(\mathcal{A})=\chi_{t}(\mathcal{B})$ ), there is an ideal $\mathcal{A}^{\prime} \subset$ $\mathcal{B}\left(\mathcal{A}^{\prime}=\mathcal{B}\right)$ for which $\chi\left(\mathcal{A}^{\prime}\right)=\chi(\mathcal{A})$ and $\chi_{t}\left(\mathcal{A}^{\prime}\right)=\chi_{t}(\mathcal{A})$ (this may be deduced e.g. from (23-3); $\mathcal{A}^{\prime}$ may be defined as $\mathfrak{J}[F]$ for suitable clopen set $F \subset \operatorname{supp}_{\Omega} \mathcal{B}$ ). Now Theorem 17.12 (page 63) combined with (23-3) implies that there are homeomorphisms $h_{D}: \Omega_{D} \cap \operatorname{supp}_{\Omega} \mathcal{A} \rightarrow$ 
$\Omega_{D} \cap \operatorname{supp}_{\Omega} \mathcal{A}^{\prime}$ where $D$ runs over $I_{1}, I_{2}, \ldots, I_{\infty}, I I_{1}, I I_{\infty}, I I I$. Define a homeomorphism $H: \operatorname{supp}_{\Omega} \mathcal{A} \rightarrow \operatorname{supp}_{\Omega} \mathcal{B}$ as the unique continuous extension of the union of all $h_{D}$ 's. Finally let $\Phi: \mathcal{A} \rightarrow \mathcal{B}$ be defined as follows. For $\mathbb{A} \in \mathcal{A}$ put $f=\Psi_{N}(\mathbb{A}) \in \Lambda(\Omega)$. Since $\operatorname{supp} f \subset \operatorname{supp}_{\Omega} \mathcal{A}$, the rules $g=f \circ H^{-1}$ on $\operatorname{supp}_{\Omega} \mathcal{B}$ and $g=0$ elsewhere well defines $g \in \Lambda(\Omega)$ such that $\operatorname{supp} g \subset \operatorname{supp}_{\Omega} \mathcal{B}$. We put $\Phi(\mathbb{A})=\Psi_{N^{\prime}}^{-1}(g)$. It is easily seen that $\Phi$ is a well defined bijection. What is more, $\Phi$ satisfies condition (23-2), by point (D4') of Theorem 15.2 (page 46). Consequently, Theorem 23.4 yields that $\Phi$ is an isomorphism. It follows from the construction that $\Phi$ is in fact a t-isomorphism.

23.7. Corollary. If $\mathcal{A} \preccurlyeq^{i} \mathcal{B}$ and $\mathcal{B} \preccurlyeq^{i} \mathcal{A}$, then $\mathcal{A} \cong{ }^{i} \mathcal{B}$.

23.8. Corollary. $\mathcal{C D} \mathcal{D}_{N} \cong t \mathcal{J}(1)$ where $\mathcal{J}(1) \subset \mathcal{C D D}$ is the ideal of all contraction operators.

Proof. Thanks to Theorem 23.6 we may assume that $N=1$. Observe that the $\mathfrak{b}$-transform is a t-isomorphism of $\mathcal{C D D}$ onto a subideal of $\mathcal{J}(1)$. So, the assertion follows from Corollary 23.7.

23.9. Corollary. Let $\mathcal{U} \subset \mathcal{C D D}$ be the ideal of all unitary operators.

(1) $\mathcal{J}_{I_{1}} \subset \mathcal{C D D}_{N}$, the ideal of all normal $N$-tuples, is t-isomorphic to $\mathcal{U}$.

(2) $\mathcal{J}_{I} \subset \operatorname{CDD}_{N}$, the ideal of all $N$-tuples of type $I$, is d-isomorphic to $\mathcal{U}$.

Proof. To see (1), repeat the argument in the proof of Corollary 23.8. To show (2), observe that all entries of the suitable characteristics of both the ideals $\mathcal{J}_{1}$ and $\mathcal{U}$ are equal to $2^{\aleph_{0}}$ and apply Theorem 23.6.

The above corollaries say that whatever can be said about single (unitary) contraction operators in the language of 'discrete' direct sums, this will have its natural 'counterpart' for arbitrary (type I) $N$-tuples.

23.10. Remark. Since $\mathcal{C D} \mathcal{D}_{N} \cong{ }^{t} \mathcal{C D} \mathcal{D}_{N^{\prime}}$ for any $N$ and $N^{\prime}$, we may also speak of spatially i-isomorphic ideals. Precisely, ideals $\mathcal{A} \subset \mathcal{C D} \mathcal{D}_{N}$ and $\mathcal{A}^{\prime} \subset \mathcal{C D D}_{N^{\prime}}$ are spatially i-isomorphic (as usual, 'i' is the empty, 's' or 't' prefix) iff there is an i-isomorphism $\Phi: \mathcal{C D D}_{N} \rightarrow \mathcal{C D} \mathcal{D}_{N^{\prime}}$ which sends $\mathcal{A}$ onto $\mathcal{A}^{\prime}$. However, this idea brings nothing new. Indeed, it is quite easy to check that $\mathcal{A}$ and $\mathcal{A}^{\prime}$ are spatially i-isomorphic iff $\mathcal{A} \cong{ }^{i} \mathcal{A}^{\prime}$ and $\mathcal{A}^{\perp} \cong{ }^{i}\left(\mathcal{A}^{\prime}\right)^{\perp}$. So, we only have to double the length of characteristics. One information in this subject however may be interesting: up to spatial isomorphim, there is only $\operatorname{card}\left(\left\{\alpha \in\right.\right.$ Card: $\left.\left.\alpha \leqslant 2^{\aleph_{0}}\right\}\right)$ different ideals. So, under the continuum hypothesis, this number is countable. 


\section{Concluding Remarks}

A. Results of Sections 20-22, especially Lemmas 20.9 (page 83) and 22.12 (page 102), prove that it is good to know how to recognize regularity measures. Especially in finite-dimensional case, since Proposition 20.2 (page 77) simply characterizes summable fields of $N$-tuples. The author is not aware of the existence of any result in this direction. We suppose that

Conjecture. Every $\sigma$-finite (Borel) measure on $\mathfrak{a}_{N}(n)$ for finite $n$ is concentrated on a measurable domain.

Below we answer the conjecture in the affirmative for $n=1$. (We are convinced this is well known. However, we could not find anything about this in the literature.) Let us first make some comments on consequences of the conjecture. If only it is true, every pair $\left(\mathscr{X},\left\{\mathbb{P}_{x}\right\}_{x \in \mathscr{X}}\right)$ where $(\mathscr{X}, \mathcal{M}, \mathcal{N})$ is standard and $\mathscr{X} \ni x \mapsto \mathbb{P}_{x} \in \bigcup_{n=1}^{\infty} \mathfrak{a}_{N}(n)$ is a one-to-one Borel function is a regular system, i.e. $\mathbb{P}_{x}$ 's 'form' the prime decomposition of some $\mathbb{X} \in \mathcal{S} \mathcal{E} \mathcal{P}_{N}$. Indeed, the sets $\mathscr{X}_{n}=\{x \in$ $\left.\mathscr{X}: \operatorname{dim}\left(\mathbb{P}_{x}\right)=n\right\}(n=1,2, \ldots)$ are measurable and there is a finite Borel measure $\mu_{n}$ on $\mathfrak{a}_{N}(n)$ such that the assignment $\mathscr{X}_{n} \ni x \mapsto$ $\mathbb{P}_{x} \in \mathfrak{a}_{N}(n)$ is an almost null-isomorphism between $\left(\mathscr{X}_{n},\left.\mathfrak{M}\right|_{\mathscr{X}_{n}},\left.\mathcal{N}\right|_{\mathscr{X}_{n}}\right)$ and $\left(\mathfrak{a}_{N}(n), \mathfrak{B}\left(\mathfrak{a}_{N}(n)\right), \mathcal{N}\left(\mu_{n}\right)\right)$. Now it follows from the conjecture that $\mu_{n} \in \operatorname{rgm}\left(\mathfrak{a}_{N}(n)\right)$ and consequently $\left\{\mathbb{P}_{x}\right\}_{x \in \mathscr{X}_{n}} \in \mathrm{RGS}_{\text {loc }}$. Put $\mathbb{X}_{n}=\boxplus_{x \in \mathscr{X}_{n}}^{\mathcal{N}} \mathbb{P}_{x}\left(=\int_{\mathfrak{a}_{N}(n)}^{\boxplus} \mathbb{P} \mathrm{d} \mu_{n}(\mathbb{P})\right)$. We conclude from Corollary 22.7 (page 99) that $\mathbb{X}_{n} \in \mathcal{J}_{I_{n}}$. So, $\mathbb{X}_{n} \perp_{u} \mathbb{X}_{m}$ for $n \neq m$ and therefore $\mu_{n} \perp_{s} \mu_{m}$, thanks to Lemma 20.10 (page 85). Now it suffices to apply Lemma 22.12 (page 102) to obtain that $\left\{\mathbb{P}_{x}\right\}_{x \in \mathscr{X}} \in \mathrm{RGS}_{l o c}$ (and $\left.\boxplus_{n=1}^{\infty} \mathbb{X}_{n}=\boxplus_{x \in \mathscr{X}}^{\mathcal{N}} \mathbb{P}_{x}\right)$.

Let us add here that the work of Ernest shows that there are standard Borel measures on $\mathfrak{p}_{N} \cap \mathcal{S E} \mathcal{E} \mathcal{P}_{N}(\infty)$ which are not concentrated on measurable domains (see Propositions 1.53 and 3.13 in [9]).

Let us now show that every $\sigma$-finite Borel measure $\mu$ on $\mathbb{C}^{N}$ is concentrated on a measurable domain. Since there is a finite Borel measure $\nu$ on $\mathbb{C}^{N}$ such that $\mu \ll \nu$, we may assume $\mu$ is finite. First assume $\mu$ is concentrated on a compact set. Put $\boldsymbol{T}=\int_{\mathbb{C}^{N}}^{\oplus} \xi \mathrm{d} \mu(\xi)$. It follows from the Stone-Weierstrass theorem that $M_{f} \in \mathcal{W}(\boldsymbol{T})$ for every $f \in \mathcal{C}(K)$ where $M_{f}$ is the multiplication operator by $f$. This implies that $M_{u} \in \mathcal{W}(\boldsymbol{T})$ for every $u \in L^{\infty}(\mu)$ as well. Consequently, $M_{u} \in \mathcal{Z}(\mathcal{W}(\boldsymbol{T})$ ) (since $\mathcal{W}(\boldsymbol{T})$ consists of decomposable operators) and hence $\int_{A}^{\oplus} \xi \mathrm{d} \mu(\xi) \perp_{u} \int_{\mathbb{C}^{N} \backslash A}^{\oplus} \xi \mathrm{d} \mu(\xi)$ which shows that $\mathbb{T}=\int_{\mathbb{C}^{N}}^{\boxplus} \xi \mathrm{d} \mu(\xi)$ and thus $\mu \in \operatorname{rgm}\left(\mathbb{C}^{N}\right)$.

Now if $\mu$ is arbitrary, there is a sequence $\left(K_{n}\right)_{n=1}^{\infty}$ of compact pairwise disjoint subsets of $\mathbb{C}^{N}$ such that $\mu\left(\mathbb{C}^{N} \backslash \bigcup_{n=1}^{\infty} K_{n}\right)=0$. The above argument proves that $\left.\mu\right|_{K_{n}} \in \operatorname{rgm}\left(\mathbb{C}^{N}\right)$ for every $n$. Put $\mathbb{X}_{n}=$ $\int_{K_{n}}^{\boxplus} \xi \mathrm{d} \mu(\xi)$. Now we repeat earlier argument: $\mathbb{X}_{n} \perp_{u} \mathbb{X}_{m}$ for $n \neq m$ (by 
Lemma 20.10, page 85) and thus $\mu \in \operatorname{rgm}\left(\mathbb{C}^{N}\right)$, thanks to Lemma 22.12 (page 102).

B. Theorem 22.17 (page 106; cf. also Remark 22.8, page 100) establishes a one-to-one correspondence between coverings and functions $\mu: \mathfrak{M} \rightarrow \mathcal{C D D}_{N}$ satisfying conditions (M0)-(M3) (see the statement of Theorem 22.17). These conditions are purely 'discrete', i.e. they need no measure-theoretic nor topological background and are formulated in terms of the direct sum operation of a pair. So, it seems to be interesting (and may turn out to be relevant) which topological or measure-theoretic notions (operations, features, tools, etc.) are sufficient for reconstructing from $\mu$ the covering to which it corresponds.

C. Similarly as we defined continuous direct sums, one may try to define 'continuous' ideals in $\mathcal{C D} \mathcal{D}_{N}$. It may be done in at least a few ways. Here we propose only one of them. Let us call an ideal $\mathcal{A} \subset \mathcal{C D} \mathcal{D}_{N}$ continuous if $\mathcal{A}$ satisfies the following condition. Whenever $(\mathscr{X}, \mathfrak{M}, \mathcal{N}, \Phi)$ is a full covering and $\mathbb{A}=\boxplus_{x \in \mathscr{X}}^{\mathcal{N}} \mathfrak{m}(x) \odot \Phi(x)$ for some $\mathfrak{m} \in \mathscr{A}(\mathscr{X})$, then $\mathbb{A} \in \mathcal{A}$ if and only if there is a set $\mathscr{Z} \in \mathcal{N}$ such that $\Phi(x) \in \mathcal{A}$ for every $x \in s(\mathfrak{m}) \backslash \mathscr{Z}$. Using Theorem 22.14 (page 104) one may easily check that it suffices to verify the above condition with a one fixed full covering and only for $\mathbb{A} \in \mathcal{S} \mathcal{E} \mathcal{P}_{N}$. For example, $\mathcal{J}_{i}$ is a continuous ideal for each $i \in\left\{I, I_{1}, I_{2}, \ldots, I_{\infty}, I I, I I_{1}, I I_{\infty}, I I I\right\}$, while $\mathcal{J}_{i}^{c}$ and $\mathcal{J}_{i}^{d}$ are not. A $p$ isomorphism (the prefix 'p' stands for 'prime decomposition') between continuous ideals is such an isomorphism $\Psi: \mathcal{A} \rightarrow \mathcal{B}$ that whenever $\mathbb{A}=\boxplus_{x \in \mathscr{X}}^{\mathcal{N}} \mathfrak{m}(x) \odot \mathbb{P}_{x}$ is a prime decomposition of $\mathbb{A} \in \mathcal{A}$, prime decomposition of $\Psi(\mathbb{A})$ may be written in the form $\bigoplus_{x \in \mathscr{X}}^{\mathcal{N}} \mathfrak{m}(x) \odot \Psi\left(\mathbb{P}_{x}\right)$, and the same for $\Psi^{-1}$. The following problem may be interesting.

Question. Are $\mathrm{CDD}_{N}$ and $\mathrm{CDD}_{N^{\prime}}$ p-isomorphic?

D. Our last remark is about the length of tuples. Readers interested in sequences (that is, countable infinite families) of closed densely defined operators acting in common Hilbert spaces may verify that most of the results (with no changes in proofs) of this treatise remain true also in that case, i.e. for $N=\infty$. (However, when working with uncountable families, a counterpart of crucial Theorem 3.4, page 8, fails to be true which causes that the whole theory crashes in that case.) Since infinite sequences are rarely investigated, we restricted our study to finite collections.

\section{REFERENCES}

[1] B. Blackadar, Operator Algebras. Theory of $\mathcal{C}^{*}$-algebras and von Neumann algebras (Encyclopaedia of Mathematical Sciences, vol. 122: Operator Algebras and Non-Commutative Geometry III), Springer-Verlag, Berlin-Heidelberg, 2006.

[2] A. Brown, On a class of operators, Proc. Amer. Math. Soc. 4 (1953), 723-728. 
[3] A. Brown, C.-K. Fong, D.W. Hadwin, Parts of operators on Hilbert space, Illinois J. Math. 22 (1978), 306-314.

[4] C. Castaing, Quelques problèmes de mesurabilité liées à la théorie de la commande, C. R. Paris 262 (1966), 409-411.

[5] X. Catepillán, M. Ptak, W. Szymański, Multiple canonical decompositions of families of operators and a model of quasinormal families, Proc. Amer. Math. Soc. 121 (1994), 1165-1172.

[6] E.G. Effros, The Borel space of von Neumann algebras on a separable Hilbert space, Pacific J. Math. 15 (1965), 1153-1164.

[7] E.G. Effros, Global structure in von Neumann algebras, Trans. Amer. Math. Soc. 121 (1966), 434-454.

[8] R. Engelking, General Topology. Revised and completed edition (Sigma series in pure mathematics, vol. 6), Heldermann Verlag, Berlin, 1989.

[9] J. Ernest, Charting the operator terrain, Mem. Amer. Math. Soc. 171 (1976), $207 \mathrm{pp}$.

[10] M. Fujii, M. Kajiwara, Y. Kato, F. Kubo, Decompositions of operators in Hilbert spaces, Math. Japon. 21 (1976), 117-120.

[11] L. Ge and J. Shen, Generator problem for certain property $T$ factors, 99 (2002), 565-567.

[12] L. Gillman and M. Jerison, Rings of continuous functions, New York, 1960.

[13] E.L. Griffin Jr., Some contributions to the theory of rings of operators, Trans. Amer. Math. Soc. 75 (1953), 471-504.

[14] E.L. Griffin Jr., Some contributions to the theory of rings of operators II, Trans. Amer. Math. Soc. 79 (1955), 389-400.

[15] D.W. Hadwin, An operator-valued spectrum, Notices Amer. Math. Soc. 23 (1976), A-163.

[16] D.W. Hadwin, An operator-valued spectrum, Indiana Univ. Math. J. 26 (1977), 329-340.

[17] P.R. Halmos and J.E. McLaughlin, Partial isometries, Pacific J. Math. 13 (1963), 585-596.

[18] R.V. Kadison and J.R. Ringrose, Fundamentals of the Theory of Operator Algebras. Volume I: Elementary Theory, Academic Press, Inc., New YorkLondon, 1983.

[19] R.V. Kadison and J.R. Ringrose, Fundamentals of the Theory of Operator Algebras. Volume II: Advanced Theory, Academic Press, Inc., Orlando-London, 1986.

[20] I. Kaplansky, A theorem on rings of operators, Pacific J. Math. 1 (1951), 227232.

[21] J.S. Kim, Ch.R. Kim, S.G. Lee, Reducing operator valued spectra of a Hilbert space operator, J. Korean Math. Soc. 17 (1980), 123-129.

[22] K. Kuratowski and A. Mostowski, Set Theory with an Introduction to Descriptive Set Theory, PWN - Polish Scientific Publishers, Warszawa, 1976.

[23] R.I. Loebl, A note on containment of operators, Bull. Austral. Math. Soc. 33 (1986), 279-291.

[24] J. von Neumann, Zur Algebra der Funktionaloperationen und Theorie der normalen Operatoren, Math. Ann. 102 (1930), 370-427.

[25] J. von Neumann, On Rings of Operators. Reduction Theory, Ann. Math. 50 (1949), 401-485.

[26] O. Nielson, Borel sets of von Neumann algebras, Amer. J. Math. 95 (1973), 145-164.

[27] H.L. Royden, Real Analysis, The Macmillan Co., New York, 1963. 
[28] T. Saitô, Generations of von Neumann algebras, Lecture Notes in Math. vol. 247 (Lecture on Operator Algebras), Springer, Berlin-Heidelberg-New York, 1972, 435-531.

[29] S. Sakai, $\mathcal{C}^{*}$-Algebras and $\mathcal{W}^{*}$-Algebras, Springer-Verlag, Berlin-HeidelbergNew York, 1971.

[30] J.T. Schwartz, $\mathcal{W}^{*}$-algebras, Gordon and Breach, Science Publishers Inc., New York-London-Paris, 1967.

[31] D. Sherman, On the dimension theory of von Neumann algebras, Math. Scand. 101 (2007), 123-147.

[32] M. Słociński, On the Wold-type decomposition of a pair of commuting isometries, Ann. Polon. Math. 37 (1980), 255-262.

[33] J. Stochel and F.H. Szafraniec, The normal part of an unbounded operator, Nederl. Akad. Wetensch. Proc. Ser. A 92 (1989), 495-503.

[34] M. Takesaki, Theory of Operator Algebras I (Encyclopaedia of Mathematical Sciences, Volume 124), Springer-Verlag, Berlin-Heidelberg-New York, 2002.

[35] M. Takesaki, Theory of Operator Algebras II (Encyclopaedia of Mathematical Sciences, Volume 125), Springer-Verlag, Berlin-Heidelberg-New York, 2003.

[36] J. Tomiyama, Generalized dimension function for $\mathcal{W}^{*}$-algebras of infinite type, Tôhoku Math. J. (2) 10 (1958), 121-129.

[37] W. Wogen, On generators for von Neumann algebras, Bull. Amer. Math. Soc. 75 (1969), 95-99.

Piotr Niemiec, Jagiellonian University, Institute of Mathematics, Ul. Lojasiewicza 6, 30-348 Kraków, Poland

E-mail address: piotr.niemiec@uj.edu.pl 